\title{
The earliest phases of high-mass star formation, as seen in NGC 6334 by Herschel-HOBYS $\star, \star \star$
}

\author{
J. Tigé ${ }^{1}$, F. Motte ${ }^{2,3}$, D. Russeil ${ }^{1}$, A. Zavagno ${ }^{1}$, M. Hennemann ${ }^{4}$, N. Schneider ${ }^{5,6}$, T. Hill ${ }^{7}$, Q. Nguyen Luong ${ }^{8,9}$, \\ J. Di Francesco ${ }^{10,11}$, S. Bontemps ${ }^{5}$, F. Louvet ${ }^{12}$, P. Didelon ${ }^{3}$, V. Könyves ${ }^{3}$, Ph. André ${ }^{3}$, G. Leuleu ${ }^{1}$, J. Bardagi ${ }^{1}$, \\ L. D. Anderson ${ }^{13,14}$, D. Arzoumanian ${ }^{3}$, M. Benedettini ${ }^{15}$, J.-P. Bernard ${ }^{16}$, D. Elia ${ }^{15}$, M. Figueira ${ }^{1}$, J. Kirk ${ }^{17}$, \\ P. G. Martin ${ }^{18}$, V. Minier ${ }^{3}$, S. Molinari ${ }^{15}$, T. Nony ${ }^{2}$, P. Persi ${ }^{15}$, S. Pezzuto ${ }^{15}$, D. Polychroni ${ }^{19}$, T. Rayner ${ }^{20}$, \\ A. Rivera-Ingraham ${ }^{21}$, H. Roussel ${ }^{22}$, K. Rygl ${ }^{23}$, L. Spinoglio ${ }^{13}$, and G. J. White ${ }^{24,25}$
}

(Affiliations can be found after the references)

Received 24 May 2016 / Accepted 14 March 2017

\begin{abstract}
Aims. To constrain models of high-mass star formation, the Herschel-HOBYS key program aims at discovering massive dense cores (MDCs) able to host the high-mass analogs of low-mass prestellar cores, which have been searched for over the past decade. We here focus on NGC 6334, one of the best-studied HOBYS molecular cloud complexes.

Methods. We used Herschel/PACS and SPIRE 70-500 $\mu$ m images of the NGC 6334 complex complemented with (sub)millimeter and mid-infrared data. We built a complete procedure to extract $\sim 0.1 \mathrm{pc}$ dense cores with the getsources software, which simultaneously measures their far-infrared to millimeter fluxes. We carefully estimated the temperatures and masses of these dense cores from their spectral energy distributions (SEDs). We also identified the densest pc-scale cloud structures of NGC 6334, one $2 \mathrm{pc} \times 1 \mathrm{pc}$ ridge and two $0.8 \mathrm{pc} \times 0.8 \mathrm{pc}$ hubs, with volume-averaged densities of $\sim 10^{5} \mathrm{~cm}^{-3}$.

Results. A cross-correlation with high-mass star formation signposts suggests a mass threshold of $75 M_{\odot}$ for MDCs in NGC 6334. MDCs have temperatures of $9.5-40 \mathrm{~K}$, masses of 75-1000 $M_{\odot}$, and densities of $1 \times 10^{5}-7 \times 10^{7} \mathrm{~cm}^{-3}$. Their mid-infrared emission is used to separate 6 IR-bright and 10 IR-quiet protostellar MDCs while their $70 \mu \mathrm{m}$ emission strength, with respect to fitted SEDs, helps identify 16 starless MDC candidates. The ability of the latter to host high-mass prestellar cores is investigated here and remains questionable. An increase in mass and density from the starless to the IR-quiet and IR-bright phases suggests that the protostars and MDCs simultaneously grow in mass. The statistical lifetimes of the high-mass prestellar and protostellar core phases, estimated to be $1-7 \times 10^{4} \mathrm{yr}$ and at most $3 \times 10^{5} \mathrm{yr}$ respectively, suggest a dynamical scenario of high-mass star formation.

Conclusions. The present study provides good mass estimates for a statistically significant sample, covering the earliest phases of high-mass star formation. High-mass prestellar cores may not exist in NGC 6334, favoring a scenario presented here, which simultaneously forms clouds, ridges, MDCs, and high-mass protostars.
\end{abstract}

Key words. dust, extinction - ISM: clouds - stars: formation - submillimeter: ISM - stars: protostars - ISM: individual objects: NGC 6334

\section{Introduction}

High-mass (O-B3 type, $\left.M_{\star}>8 M_{\odot}\right)$ stars form in massive dense cores (MDCs, $\sim 0.1 \mathrm{pc}$ and $>10^{5} \mathrm{~cm}^{-3}$, see Motte et al. 2007 ) by the accretion of gas onto stellar embryos (e.g., Beuther \& Schilke 2004; Duarte-Cabral et al. 2013). The details of this process are under investigation (see recent reviews by, e.g., Tan et al. 2014; Krumholz 2015; Motte et al. 2017) and an increasing number of studies have suggested that they form through dynamical processes initiated by cloud formation (e.g., Schneider et al. 2010a; Csengeri et al. 2011; Peretto et al. 2013; Nguyen-Luong et al. 2013).

Two main theoretical scenarios have been proposed to explain the formation of high-mass stars: (1) powerful accretion driven by a high degree of turbulence (e.g., McKee \& Tan 2002; Krumholz et al. 2007) or (2) colliding flows initiated by

\footnotetext{
* Herschel is an ESA space observatory with science instruments provided by European-led Principal Investigator consortia and with important participation from NASA.

$\star \star$ Catalogs built from Tables A.1-A.12, are only available at the CDS via anonymous ftp to cdsarc.u-strasbg. fr (130.79.128.5) or via http://cdsarc.u-strasbg.fr/viz-bin/qcat?]/A+A/602/A77
}

competitive accretion or cloud formation (e.g., Bonnell \& Bate 2006; Hartmann et al. 2012). These two scenarios lead to distinct characteristics for the initial stages of high-mass star formation. The first model implies the existence of high-mass prestellar cores, which display high degrees of micro-turbulence. In the second model, high-mass prestellar cores never develop. When favorably located at the centers of massive reservoirs of gas, lowmass prestellar cores turn into protostars, attracting gas from further distances and eventually become high-mass protostars. Therefore, one difference between the two scenarios is the presence or absence of high-mass prestellar cores.

A decade ago, Motte et al. (2007) made the first unbiased statistical survey of MDCs. They did not find any bona-fide starless MDCs and proposed that this phase would be transitory if existent. Since then, ground-based studies have only been able to identitfy a handful of starless MDCs in molecular complexes (e.g., Russeil et al. 2010; Butler \& Tan 2012) and only a few hundred starless clumps in the Milky Way (Ginsburg et al. 2012; Tackenberg et al. 2012; Csengeri et al. 2016; Svoboda et al. 2016). A few high-resolution studies have been performed with (sub)millimeter interferometers with the aim of identifying individual prestellar cores and protostars 
within MDCs. These attempts revealed high-mass cores that are protostellar in nature (e.g., Duarte-Cabral et al. 2013, within protostellar MDCs) or low-mass prestellar fragments (e.g., Tan et al. 2013, within starless clumps). The only prestellar core candidate of Duarte-Cabral et al. (2014, Cyg X-N53-MM2, $25 M_{\odot}$ mass within $0.025 \mathrm{pc}$ ) and the single case found by Wang et al. 2014, G11P6-SMA1, $30 M_{\odot}$ mass within $0.02 \mathrm{pc}$ ) are amongst the very few good examples to date of the massive prestellar cores predicted by McKee \& Tan (2002). This fact alone argues for a high-mass star formation scenario with no high-mass prestellar core phase (see Motte et al. 2017) but the debate continues.

As a first step to resolving the debate, it is crucial to find and characterize MDCs that are potential sites for the formation of high-mass stars, in molecular cloud complexes. The Herschel imaging survey of OB Young Stellar Objects (HOBYS) is dedicated to this purpose (Motte et al. 2010) ${ }^{1}$. It is the first systematic survey of a complete sample of nearby (within $3 \mathrm{kpc}$ ) high-mass star progenitors at $0.1 \mathrm{pc}$ scales (see initial catalogs in Nguyen Luong et al. 2011; Fallscheer et al. 2013). At five wavelengths from $70 \mu \mathrm{m}$ to $500 \mu \mathrm{m}$, the HOBYS program targeted ten molecular cloud complexes forming OB-type stars, amongst which NGC $6334-6357$ is one of the most massive $\left(\sim 7 \times 10^{5} \mathrm{M}_{\odot}\right.$, see Motte et al. 2017).

NGC 6334-6357 is a molecular cloud complex that belongs to the Sagittarius-Carina arm and lies at a $1.75 \mathrm{kpc}$ distance from the Sun (Matthews et al. 2008; Russeil et al. 2013). Its central part hosts a 15 pc-long filament (Russeil et al. 2013; Schneider et al. 2015), whose highest density parts consist of a ridge according to HOBYS terminology (Hill et al. 2011). NGC 6334 is itself a very active high-mass-star-forming region, as advocated by its numerous H II regions, maser sources, and molecular outflows (see Loughran et al. 1986; Persi \& Tapia 2008; Carral et al. 2002). Willis et al. (2013) identified approximately 2000 young stellar objects with Spitzer and found a heavy concentration of protostars along the NGC 6334 ridge, suggesting a mini-starburst event (in agreement with the ridge definition, see Nguyen Luong et al. 2011). Using a $1.2 \mathrm{~mm}$ continuum map, Russeil et al. (2010) extracted in NGC 6334 a sample of 11 clumps, including the well-known sources I and I(N) (e.g., Gezari 1982), however, only one starless candidate was identified.

In this paper, we search the NGC 6334 complex for MDCs harboring young stellar objects at the earliest phases of highmass-star formation, that is, high-mass young protostars and high-mass prestellar cores. From Herschel and complementary images described in Sect. 2, we extracted ridges, hubs, and dense cores (see Sect. 3). We describe the SED characterization of these cores in Sect. 4. Section 5 provides a complete sample of 0.1 pc MDCs with robust mass estimates. Finally, in Sect. 6, we discuss the existence of starless MDCs and prestellar cores, the lifetimes of high-mass star formation phases, and the favored scenario for high-mass-star formation.

\section{Observations}

\subsection{Herschel observations, data reduction, and column density images}

NGC 6334 was observed by the Herschel space observatory with the PACS (Poglitsch et al. 2010) and SPIRE (Griffin et al. 2010)

\footnotetext{
1 See http://hobys-herschel.cea.fr
}

Table 1. Main observational parameters of the present paper.

\begin{tabular}{c|c|c|c}
\hline \hline Data & $\begin{array}{c}\lambda \\
(\mu \mathrm{m})\end{array}$ & $\begin{array}{c}\text { HPBW } \\
(\operatorname{arcsec})\end{array}$ & $\begin{array}{c}\text { rms }(1 \sigma) \\
(\mathrm{mJy})\end{array}$ \\
\hline \multirow{2}{*}{ Herschel/PACS } & 70 & 5.9 & 90 \\
& 160 & 11.7 & 650 \\
and SPIRE & 350 & 18.2 & 1100 \\
& 500 & 24.9 & 860 \\
\hline \multirow{2}{*}{ JCMT/SCUBA-2 } & 450 & 8.5 & 680 \\
\hline APEX/LABOCA & 850 & 15.0 & 470 \\
\hline SEST/SIMBA & 1200 & 19.2 & 90 \\
\hline & 3.6 & 1.5 & 0.6 \\
Spitzer/IRAC & 4.5 & 1.7 & 0.6 \\
& 5.8 & 1.7 & 0.7 \\
and MIPS & 8 & 2.0 & 5 \\
& 24 & 6.0 & 5 \\
\hline WISE & 22 & 12.0 & 10 \\
\hline MSX & 21 & 18.3 & 60 \\
\hline
\end{tabular}

instruments $^{2}$ as part of the HOBYS (Motte et al. 2010) key program (OBSIDs: 1342204421 and 1342204422). Data were taken in five bands: $70 \mu \mathrm{m}$ and $160 \mu \mathrm{m}$ for PACS at FWHM resolutions or half power beam widths (HPBWs) of 5.9" and 11.7", respectively, and $250 \mu \mathrm{m}, 350 \mu \mathrm{m}$, and $500 \mu \mathrm{m}$ for SPIRE at FWHM resolutions of 18.2" $24.9^{\prime \prime}$, and $36.3^{\prime \prime}$, respectively (see Table 1). Observations were performed in parallel mode, using both instruments simultaneously, with a scanning speed of $20^{\prime \prime} / \mathrm{s}$. Two perpendicular scans were taken, quoted as nominal and orthogonal. The size of the observed field is $2.0^{\circ} \times 0.5^{\circ}$, which corresponds to $60 \mathrm{pc} \times 15 \mathrm{pc}$ at a distance of $1.75 \mathrm{kpc}$. Herschel images of NGC 6334 are thus sensitive to cloud structures with $\sim 0.1 \mathrm{pc}$ to $\sim 30 \mathrm{pc}$ typical sizes, which correspond to MDCs, clumps, and clouds following definitions by, for example, Motte et al. (2017).

We reduced Herschel data using the Herschel interactive processing environment (HIPE, Ott 2010) ${ }^{3}$ software, version 10.0.2751. Versions 7.0 onwards contain a module which significantly removes the striping effects that have been observed in SPIRE maps produced with previous HIPE versions. SPIRE nominal and orthogonal maps were separately processed and subsequently combined and reduced for de-striping, relative gains, and color correction with HIPE. PACS maps were reduced with HIPE up to Level 1 and, from there up to their final version (Level 3) using Scanamorphos v21.0 (Roussel 2013). Final PACS images are shown in Figs. C.1 and C.2.

To correct for a few saturated pixels observed in SPIRE images, individual SPIRE mappings of small fields were performed with a different gain setting. The original map with saturated pixels and the small field were then combined following the method

2 Instrument parameters and calibration are given in the PACS and SPIRE observers manuals. See http://Herschel.esac.esa.int/ Docs/PACS/html/pacs_om.html for PACS and http://Herschel. esac.esa.int/Docs/SPIRE/html/spire_handbook.html for SPIRE.

3 HIPE has been jointly developed by the Herschel Science Ground Segment Consortium, consisting of ESA, the NASA Herschel Science Center, and the HIFI, PACS, and SPIRE consortia. 


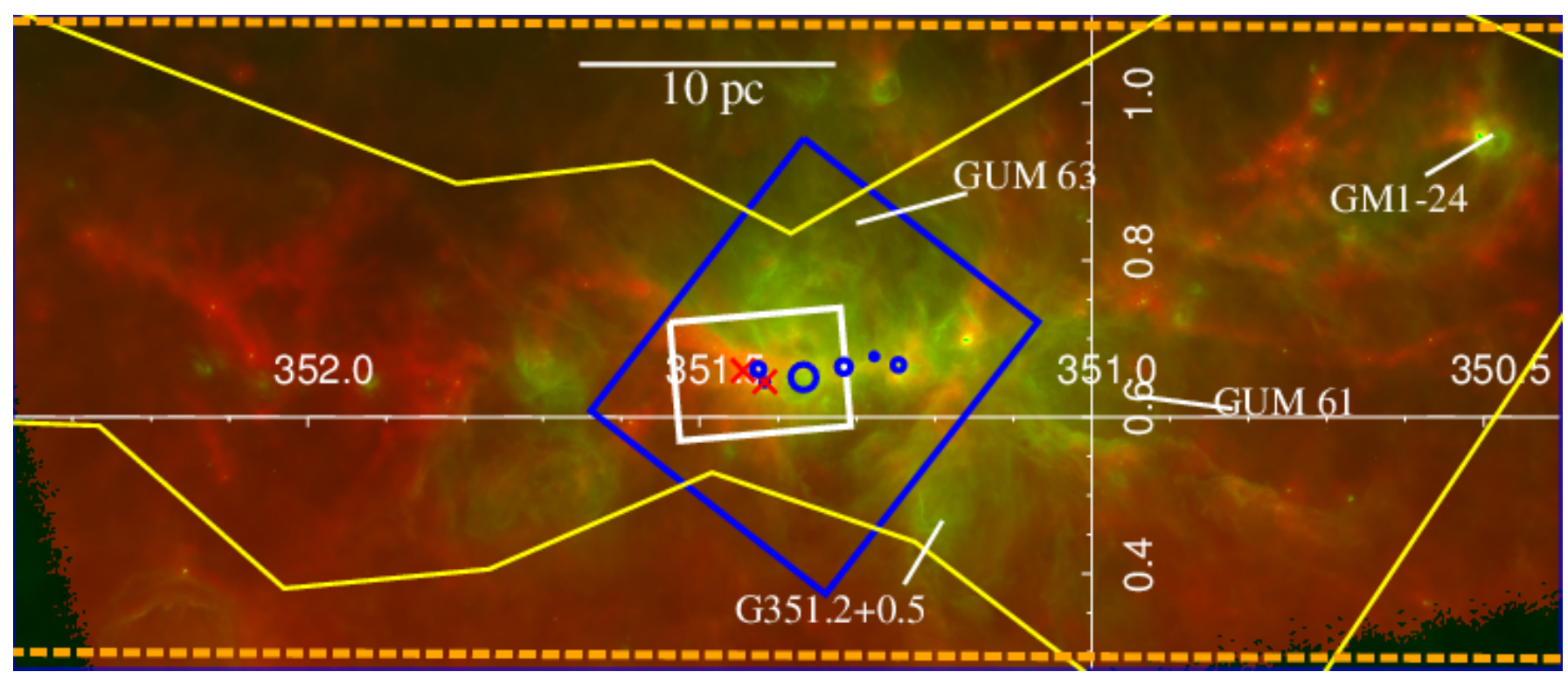

Fig. 1. Herschel-color image of NGC 6334: $70 \mu \mathrm{m}$ (green, 5.9" resolution) and column density (red, 36.6" resolution). Blue circles are young H II regions (seen as compact radio sources; Persi \& Tapia 2010) while crosses are I and I(N), the two highest column density peaks of NGC 6334. The yellow polygon represents the mapped area of SIMBA- $1200 \mu \mathrm{m}$ observations, the white box represents the extent of SCUBA2 $450 / 850 \mu \mathrm{m}$ observations, the blue box highlights the highest-density parts of NGC 6334 presented in Sect. 3.1 and Fig. 2, and dashed orange lines outline the Galactic latitude range we associated with NGC $6334\left(0.3^{\circ}<\right.$ latitude $\left.<1.1^{\circ}\right)$ to build the HOBYS catalog.

described in Appendix B of Nguyen-Luong et al. (2013) to produce full images without saturation (see Figs. C.3-C.5). Calibration for all Herschel maps was done using the offsets obtained following the procedure described in Bernard et al. (2010). This method makes use of IRIS (IRAS survey update) and Planck HFI DR2 data (Planck Collaboration I 2011) to predict an expected flux, which is compared to the median values from PACS and SPIRE maps, smoothed to the adequate resolution.

We built column density maps both at the $36.3^{\prime \prime}$ and $18.2^{\prime \prime}$ resolutions of SPIRE $500 \mu \mathrm{m}$ and $250 \mu \mathrm{m}$ data (see Figs. 1 and C.6). The procedure used to construct the $36.3^{\prime \prime}$ resolution image uses the SED fit method fully described in Hill et al. (2011, 2012). The one used to construct the high-resolution columndensity map is based on a multi-scale decomposition of the imaging data described in detail in Palmeirim et al. (2013). We used a dust opacity law similar to that of Hildebrand (1983) but with $\beta=2$ instead of $\beta=1$ and assumed a gas-to-dust ratio of 100: $\kappa_{0}=0.1 \times(v / 1000 \mathrm{GHz})^{2} \mathrm{~cm}^{2} \mathrm{~g}^{-1}$.

Figure 1 presents a combined image of Herschel $70 \mu \mathrm{m}$ and column density images of the NGC 6334 molecular complex. While the column density is concentrated along a filamentary structure, described in Russeil et al. (2013) for example, the $70 \mu \mathrm{m}$ emission traces warm dust toward star-forming sites such as $\mathrm{H}$ II regions and young stars.

\subsection{Ancillary data}

We complemented our Herschel observations with submillimeter and mid-infrared data (see Table 1). Multiple SCUBA2/JCMT $450 \mu \mathrm{m}$ and $850 \mu \mathrm{m}$ images (Holland et al. 2013) toward the I and I(N) sources of NGC 6334 were taken from the JCMT Science Archive 4 (Proposal ID: JCMTCAL). We reduced the data following the standard pipeline using the makemap command of the SMURF software (Chapin et al. 2013), bundled

4 SCUBA-2 data from the JCMT Science Archive are available at http://www. cadc-ccda.hia-iha.nrc-cnrc.gc.ca/en/jcmt/ with the Gaia package. We made two mosaics from all calibrated $450 \mu \mathrm{m}$ and $850 \mu \mathrm{m}$ data and obtained a $0.2^{\circ} \times 0.2^{\circ} \mathrm{cov}-$ erage (see Figs. 1 and C.7-C.8). The angular resolutions were $8.5^{\prime \prime}$ at $450 \mu \mathrm{m}$ and $15.0^{\prime \prime}$ at $850 \mu \mathrm{m}$. The ATLASGAL survey $^{5}$ (Schuller et al. 2009), using the LABOCA/APEX camera at $870 \mu \mathrm{m}$ itself covered the NGC 6334 molecular cloud with 19.2" resolution. Dedicated SIMBA/SEST $1.2 \mathrm{~mm}$ observations presented by Muñoz et al. (2007) and Russeil et al. (2010) also covered the main filamentary structures of NGC 6334, with $24^{\prime \prime}$ angular resolution (see coverage in Fig. 1).

At mid-infrared wavelengths, NGC 6334 was imaged by Spitzer/IRAC and MIPS at 3.6-24 $\mu \mathrm{m}$ with $1.5^{\prime \prime}-6^{\prime \prime}$ resolutions, as part of the GLIMPSE and MIPSGAL surveys ${ }^{6}$ (Benjamin et al. 2003; Carey et al. 2009). It was also imaged by the two mid-infrared space observatories ${ }^{7}$ that are called WISE, at four bands and notably at $22 \mu \mathrm{m}$ with an angular resolution of $12^{\prime \prime}$, and $M S X$, in four bands, including $21.3 \mu \mathrm{m}$ with spatial resolution of 18.3" (Wright et al. 2010; Price et al. 2001).

\section{Identification of dense cloud structures}

We investigated the cloud structure of NGC 6334 from parsecscale clumps (see Sect. 3.1) to 0.1 pc-scale dense cores (see Sect. 3.2)

The APEX Telescope Large Area Survey of the GALaxy (ATLASGAL) provides $870 \mu \mathrm{m}$ images of the inner Galactic plane. Detailed information and reduced images are available at http://www3. mpifr-bonn.mpg.de/div/atlasgal/

6 Detailed information on the GLIMPSE and MIPSGAL surveys of the inner Galactic plane with Spitzer and reduced images are available at http://irsa.ipac.caltech.edu/data/SPITZER/

Detailed information on the WISE full-sky and MSX Galactic Plane surveys and their reduced images are available at http: //irsa.ipac.caltech.edu/Missions/wise.html and http:// irsa.ipac.caltech.edu/Missions/msx.html, respectively. 


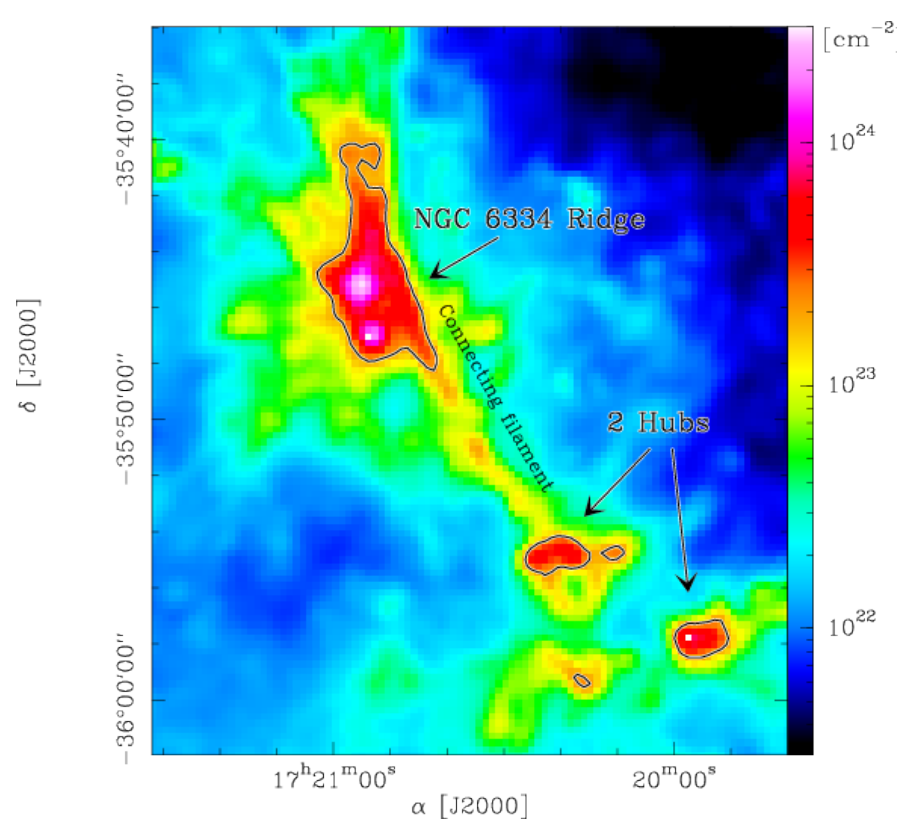

Fig. 2. NGC 6334 highest density clumps delineated in the column density image (color and contours) derived from Herschel images. The contour is the $2 \times 10^{23} \mathrm{~cm}^{-2}$ level, outlining the NGC 6334 ridge and hubs.

\subsection{The densest pc-scale clumps: ridges and hubs}

Among parsec-scale clumps, the densest ones qualify as ridges and hubs. Hill et al. (2009) first proposed to outline ridges, that is, filamentary clumps forming high-mass stars, with a $10^{23} \mathrm{~cm}^{-2}$ column density lower limit. In contrast to threshold claims by, for example, Krumholz \& McKee (2008), Hill et al. (2009) did not find any objective reason to set a definite threshold for a clump to be able to form high-mass stars. The probability distribution function (PDF) of column density in NGC 6334, studied by Schneider et al. (2015), then revealed a deviation ${ }^{8}$ from the first power-law tail at $\sim 2 \times 10^{23} \mathrm{~cm}^{-2}$, interpreted as linked to (high-mass) star formation. Figure 2 highlights the highestdensity parts of NGC 6334 on a zoomed column density image. The $2 \times 10^{23} \mathrm{~cm}^{-2}$ contours outline the NGC 6334 ridge spanning a $2 \mathrm{pc} \times 1 \mathrm{pc}$ area and containing the $\mathrm{I}$ and $\mathrm{I}(\mathrm{N})$ sources as well as two smaller hubs with $\sim 0.8$ pc diameters. A narrow filament discussed at length in Matthews et al. (2008) and André et al. (2016) connects the ridge and largest hub (see Fig. 2).

We used a $2 \times 10^{23} \mathrm{~cm}^{-2}$ column density background and Eqs. (1)-(2) of Nguyen-Luong et al. (2013) to derive a mass of $\sim 15000 M_{\odot}$ and a density of $2.5 \times 10^{5} \mathrm{~cm}^{-3}$ for the NGC 6334 ridge. With the same assumptions, the NGC 6334 hubs have masses of $\sim 2200$ and $\sim 2800 M_{\odot}$ and densities around $10^{5} \mathrm{~cm}^{-3}$.

\subsection{Compact sources}

Compact sources were extracted using getsources (v1.131121), a multi-scale, multi-wavelength source-extraction algorithm (Men'shchikov et al. 2012; Men'shchikov 2013). Extraction is

\footnotetext{
The $1 \pm 0.5 \times 10^{23} \mathrm{~cm}^{-2}$ threshold measured in the column density map of Schneider et al. (2015) becomes $2 \pm 1 \times 10^{23} \mathrm{~cm}^{-2}$ in the image of Fig. 2. This factor 2 increase arises from the change in the SED fit procedure from a linear to a logarithmic fit, which allows a better measurement of the temperature and column density of clumps and filaments strongly heated from outside. The maximum increase, 2.5, is observed along the filament connecting the NGC 6334 ridge and main hub studied in André et al. (2016).
}

based on the decomposition of images across finely spaced spatial scales. Each of these single-scale images is cleaned of noise and background emission by iterating on the appropriate cutoff levels. Images are then re-normalized and summed over all wavelengths into a combined single-scale detection image. The main advantage of this algorithm is its multi-wavelength design. Namely, using the same combined detection image across all wavelengths eliminates the need for matching multiple catalogs obtained at different wavelengths and corresponding angular resolutions. Also, the generally non-Gaussian noise spectrum of the initial maps becomes more Gaussian on single-scale images, improving detection of the faintest objects and the deblending of sources in crowded regions. The cleaning of background emission, fully explained in Men'shchikov et al. (2012), starts from global image flattening and is iteratively refined to determine precisely the local background of each individual core.

Following the procedure established by the HOBYS consortium and fully described in this paper, the detection of compact sources is based on Herschel data plus a high-resolution columndensity map. The PACS-160 $\mu \mathrm{m}$ and SPIRE-250 $\mu \mathrm{m}$ images are temperature-corrected to minimize the effects of temperature gradients. The departure to an intensity map arising from a constant temperature $(17 \mathrm{~K})$ is used to scale the original PACS$160 \mu \mathrm{m}$ and SPIRE-250 $\mu \mathrm{m}$ images, with the goal of emphasizing the cold structures. The procedure is fully explained in Appendix $\mathrm{C}$ and images are shown in Figs. C.10-C.12. The purpose of temperature-corrected maps at $160 \mu \mathrm{m}$ and $250 \mu \mathrm{m}$ is to weaken the impact of external heating, especially at the periphery of photo-dissociation regions (PDRs) to help getsources focus on cold peaks rather than temperature peaks created by local heating (see Fig. C.12).

During the detection step, we simultaneously applied getsources to all images to define a catalog of sources with a unique position. At the measurement stage, we use the original (not temperature-corrected) Herschel maps from $70 \mu \mathrm{m}$ to $500 \mu \mathrm{m}$ plus all available submillimeter maps listed in Sect. 2.2, that is, the $450 \mu \mathrm{m}$ and $850 \mu \mathrm{m}$ SCUBA-2 mosaics and the $870 \mu \mathrm{m}$ LABOCA and $1200 \mu \mathrm{m}$ SIMBA images. These measurements go beyond simple aperture photometry since they are done together with background subtraction and deblending of overlapping sources. The resulting source catalog contains a monochromatic significance index ( $\left.\operatorname{Sig}_{\text {mono }}\right)$, peak and integrated fluxes (with errors), full width at half maximum (FWHM) major and minor sizes, and the position angle of the elliptical footprint for each extracted source in each far-infrared to submillimeter band. A subset of these catalogs is given in Tables A.2-A.12.

The Herschel-HOBYS imaging encompassing NGC 6334 extends from $0^{\circ}$ to $+1.3^{\circ}$ in Galactic latitude. On the basis of $\mathrm{H} \alpha$, radio continuum, and HI surveys, Russeil et al. (2016) defined the velocity structure and thus the spatial area associated with the complex. Since we cannot confirm the association of NGC 6334 with gas located at the edges of the complex, we masked areas between $+0.0^{\circ}$ and $+0.3^{\circ}$ and between $+1.1^{\circ}$ and $+1.3^{\circ}$ in Galactic latitude (see Fig. 1). In the following, we build the first-generation catalog of NGC 6334 MDCs from the corresponding getsources catalog of 4733 sources found within the unmasked area.

Sources extracted by getsources are far-infrared fluctuations among which compact dense cores persist across wavelengths. Intermediate- to high-mass dense cores ${ }^{9}$ are expected to be cold

$0.1 \mathrm{pc}$ dense cores are larger-scale cloud structures than $0.02 \mathrm{pc}$ low-mass (prestellar or protostellar) cores assumed to form individual solar-type stars (Motte et al. 1998; Könyves et al. 2015). 
$(10-30 \mathrm{~K})$, dense cloud fragments of approximately $0.1 \mathrm{pc}$ in size and several times $1-100 M_{\odot}$ in mass. Given their expected densities, their thermal emission should mainly be optically thin for wavelengths larger than $100 \mu \mathrm{m}$.

Dense cores have spectral energy distributions (SEDs) peaking between $100 \mu \mathrm{m}$ and $300 \mu \mathrm{m}$ and should therefore have reliable flux measurements at either Herschel-160 $\mu \mathrm{m}$ or Herschel- $250 \mu \mathrm{m}$ or, more generally, at both. We define reliable flux measurements as those which have 1) signal-to-noise ratios greater than 2 for both the peak and integrated fluxes and monochromatic significance index greater than 5, and 2) deconvolved sizes less than $0.3 \mathrm{pc}$ and aspect ratios less than 2 . Sources in the getsources catalog which do not follow these criteria are either PDR features whose $70 \mu \mathrm{m}$ emission is not associated with density peaks, filaments with ellipticity $>2$, or even $\sim 1$ pc clumps. This step selects 2063 sources out of the 4733 initial getsources sources. Roughly $90 \%, 6 \%$, and $4 \%$ of the sources excluded by these criteria are $70 \mu \mathrm{m}$-only sources, filaments, and clumps, respectively.

$160 \mu \mathrm{m}$ is the highest-resolution Herschel wavelength $\left(\sim 12^{\prime \prime}\right)$ that should correctly represent the optically thin dust emission of dense cores. In some cases, however, thermal emission at $160 \mu \mathrm{m}$ can be contaminated by emission of small grains heated by PDRs at the peripheries of dense cores. One direct consequence of this effect is that deconvolved sizes can be larger at $160 \mu \mathrm{m}$ than at $250 \mu \mathrm{m}$. The size at $160 \mu \mathrm{m}$ or, if its flux is unreliable or contaminated, the size at $250 \mu \mathrm{m}$ was chosen for reference to estimate the physical size of the source. Of the dense cores, $35 \%$ have their reference wavelength set to $160 \mu \mathrm{m}$. Another $40 \%$ of the dense cores have reliable measurements at $160 \mu \mathrm{m}$, but with large deconvolved sizes their reference wavelength is set to $250 \mu \mathrm{m}$. The remaining $25 \%$ of the dense cores only have reliable measurements at $250 \mu \mathrm{m}$, which thus is their reference wavelength.

A minimum number of three reliable fluxes at wavelengths larger than $100 \mu \mathrm{m}$ are necessary to correctly constrain the SEDs of dense gas fragments. We therefore enforced a minimum of three reliable flux measurements: 1) one at either Herschel$160 \mu \mathrm{m}$ or Herschel-250 $\mu \mathrm{m}$, which we call the reference wavelength (see above), 2) accompanied by at least a second Herschel flux measurement at a wavelength larger than $100 \mu \mathrm{m}$, and 3) a third flux measurement taken at $\lambda>100 \mu \mathrm{m}$ with either Herschel/SPIRE, APEX/LABOCA, or SEST/SIMBA. In NGC 6334, this step removes more than half of the remaining getsources sources, leading to a sample of 654 dense cores. Sources excluded by this process are at the limit of our detection level in Herschel-SPIRE wavelengths and thus correspond to dense cores with very low mass.

\section{Physical characterization of dense cores}

\subsection{Coherent SEDs: fluxes scaled to the same aperture}

We aim to construct SEDs with fluxes measured within one aperture of the same size. Since the Herschel beam increases with wavelength, source sizes and thus the flux in an aperture often increase as well (see, e.g., Table 2 of Nguyen Luong et al. 2011). We correct for this bias by: 1) adding submillimeter fluxes with better angular resolution than our $500 \mu \mathrm{m}$ Herschel data (e.g., 19.2" for APEX-870 $\mu \mathrm{m}$ and 24" for SIMBA-1200 $\mu \mathrm{m}$ versus $36.6^{\prime \prime}$ for Herschel-500 $\mu \mathrm{m}$ ) and 2) scaling fluxes appropriately. The flux-scaling procedure primarily used by Motte et al. (2010) is described in more detail in Nguyen Luong et al. (2011), Giannini et al. (2012), and Fallscheer et al. (2013). In short, these HOBYS papers incorporated a simple linear scaling method that relies on the assumption that MDCs have intensity versus aperture radius profiles close to those found for highmass protostellar dense cores, $I \propto \theta^{-1}$ (e.g., Beuther et al. 2002). Scaling thus provides flux estimates as they should be when integrated within the smaller aperture of the reference wavelength, that is, $160 \mu \mathrm{m}$ or $250 \mu \mathrm{m}$. It aims at correcting integrated fluxes which mainly arise from optically thin thermal emission, in our case at $\lambda>100 \mu \mathrm{m}$. The procedure uses deconvolved sizes, $F W H M^{\text {dec }}$, integrated fluxes, $F_{\lambda>100 \mu \mathrm{m}}$, and the formula:

$$
F_{\lambda>100 \mu \mathrm{m}}^{\mathrm{corrected}}=F_{\lambda>100 \mu \mathrm{m}} \times \frac{F W H M_{\mathrm{Ref} \lambda}^{\mathrm{dec}}}{F W H M_{\lambda>100 \mu \mathrm{m}}^{\mathrm{dec}}} .
$$

The FWHM sizes estimated using getsources are equivalent to a Gaussian FWHM, and have been deconvolved assuming that the Herschel beams themselves are Gaussian. Simple Gaussian deconvolution was stopped when sources were smaller than $0.5 \times H P B W_{\lambda}$, which translates into an observed $F W H M_{\lambda}^{\text {obs }} \leq$ $1.12 \times H P B W_{\lambda}$. Unresolved sources were assigned at their reference wavelength a minimum physical size of $F W H M_{160 \mu \mathrm{m}}^{\mathrm{dec}}=$ $0.5 \times H P B W_{160 \mu \mathrm{m}} \simeq 5.85^{\prime \prime}$ or $F W H M_{250 \mu \mathrm{m}}^{\mathrm{dec}} \simeq 9.1^{\prime \prime}$, corresponding to $0.05 \mathrm{pc}$ or $0.08 \mathrm{pc}$ at $1.75 \mathrm{kpc}$. Dense cores are generally resolved at their reference wavelength but $36 \%$ are not.

When fluxes remain uncorrected, SED fits of Herschel data alone lead to, on average, twice larger masses (see, e.g., Nguyen Luong et al. 2011). The reason behind this increase is that fluxes are measured within sizes varying from the reference wavelength size to approximately twice its value when measured at $500 \mu \mathrm{m}$. Since NGC 6334 MDCs are imaged in the submillimeter regime at higher-angular resolutions than SPIRE, adding these fluxes limits this mass increase to $\sim 50 \%$. As mentioned by Nguyen Luong et al. (2011), the fitted reduced $\chi^{2}$ value is largely decreased when Herschel fluxes are linearly corrected, showing that fluxes measured in single apertures are better fitted by modified blackbody models. The linear correction is a firstorder correction method of dense core fluxes measured within varying apertures. Indeed, in the case of starless dense cores, which are less centrally concentrated than protostellar dense cores, a stronger flux-scaling correction should be applied. Since there are no theoretical laws to describe the low gas concentration of a starless structure whose fragmentation is largely governed by turbulence, for consistency we decided to keep the same flux correction for all types of dense cores. In the following, the number of starless dense cores with a given mass is probably overestimated because a mass concentration steeper than $\operatorname{Mass}(<r) \propto r$ (see Fig. 7) would lead to much smaller corrected $350 \mu \mathrm{m}$ and $500 \mu \mathrm{m}$ fluxes, higher fitted SED dust temperatures and, finally, lower derived masses.

\subsection{SED fitting procedure}

We used the MPFIT least-squares minimisation routine in IDL (Markwardt 2009) to fit the SEDs with modified blackbody models such as

$S_{v}=A v^{\beta} B_{v}\left(T_{\text {dust }}\right)$,

where $S_{v}$ is the observed flux distribution, $A$ is a scaling factor, $B_{v}\left(T_{\text {dust }}\right)$ is the Planck function for the dust temperature $T_{\text {dust }}$, and $\beta$ is the dust emissivity spectral index here set to 2 .

We assumed, for the representative error of the reliable fluxes, the quadratic sum of getsources flux errors, image 
calibration errors ${ }^{10}$, and background extraction errors. The two latter factors combined are estimated to be of the order of $10 \%$ for $70 \mu \mathrm{m}$ and $20 \%$ for fluxes at $\lambda>100 \mu \mathrm{m}$.

The $70 \mu \mathrm{m}$ flux was only used for SED fitting when the temperature fitted to $\lambda>100 \mu \mathrm{m}$ fluxes rises above $32 \mathrm{~K}$, corresponding to a modified blackbody peaking at $100 \mu \mathrm{m}$. When cloud fragments are hotter than $32 \mathrm{~K}$, their SEDs are incorrectly constrained by $\lambda>160 \mu \mathrm{m}$ data alone. The $70 \mu \mathrm{m}$ emission from the inner part of the protostellar envelope becomes larger than the extinction depth at $70 \mu \mathrm{m}$ of the modified blackbody fitted at $\lambda>100 \mu \mathrm{m}$. In such cases, the $70 \mu \mathrm{m}$ flux was therefore used as an upper limit. Throughout our sample, $20 \%$ of the sources needed the $70 \mu \mathrm{m}$ flux in their SEDs, corresponding to sources that are either IR-bright protostellar dense cores or weak sources with poorly constrained $\lambda>100 \mu \mathrm{m}$ SED portions.

Since dense cores do not have flat spectra across far-infrared to submillimeter bands, we applied color-correction factors to all Herschel fluxes. SPIRE color-correction factors depend only on the dust emissivity spectral index $\beta$, here fixed to 2 . They are $4 \%$, $4 \%$, and $5 \%$ at $250 \mu \mathrm{m}, 350 \mu \mathrm{m}$, and $500 \mu \mathrm{m}$ respectively (cf. SPIRE observers manual). PACS color-correction factors themselves vary with $T_{\text {dust }}$, especially at $70 \mu \mathrm{m}$ (Poglitsch et al. 2010). In our sample of 654 dense cores, the mean values of these corrections are $13 \%$ and $3 \%$ but they can go up to $60 \%$ and $10 \%$, at $70 \mu \mathrm{m}$ and $160 \mu \mathrm{m}$, respectively.

Since we attempted to fit all the SEDs of the 654 dense cores, even when they were very low-mass and have uncertain fluxes, we enforced fits with temperatures between $8 \mathrm{~K}$ and $70 \mathrm{~K}$. We used the fitted reduced $\chi^{2}$ value to discriminate between coherent sources with robust fits and inhomogeneous cloud structures, and qualified those with reduced $\chi^{2}<10$ as robust SEDs, and those with reduced $\chi^{2}>10$ as dubious. We found that $25 \%$ of the dense core SEDs are not robust. We excluded them in the following analysis because their parameters are too uncertain. Our fitting procedure leads to a sample of 490 dense cores for which envelope mass can be properly determined. For the remainder of the study, we consider only this sample of dense cores.

We determined the total gas + dust mass of dense cores, Mass, from their dust continuum emission, $S_{v}$, and the following equation:

Mass $=\frac{S_{v} d^{2}}{\kappa_{v} B_{v}\left(T_{\mathrm{dust}}\right)}$,

where $d$ is the distance to the Sun, $1.75 \mathrm{kpc}$, and $\kappa_{v}$ is the dust mass opacity. $\kappa_{v}$ depends on the size, shape, chemical composition, and temperature of the grains as well as the gas-to-dust mass ratio (see Ossenkopf \& Henning 1994; Martin et al. 2012; Deharveng et al. 2012). $\kappa_{v}$ thus depends on the density and temperature of the medium considered. The main assumptions used are that the source fluxes are optically thin, sources are homogeneous in density, temperature, and optical properties along the line of sight. As in Sect. 2.1, the HOBYS consortium uses the relation $\kappa_{v}=\kappa_{0}\left(v / v_{0}\right)^{\beta}$ with $\kappa_{0}=0.1 \mathrm{~cm}^{2} \mathrm{~g}^{-1}$ assuming a gasto-dust ratio of $100, v_{0}=1000 \mathrm{GHz}$ and $\beta=2$ for the dust emissivity spectral index (Hill et al. 2009; Motte et al. 2010). If we were to use $\beta=1.5$, which is the other extreme value appropriate for dense cores, temperatures and masses would differ by $5-10 \%$. We estimate that the overall uncertainty on masses measured through this procedure for dense cores with known distance and fixed size is approximately 50\% (Roy et al. 2014).

\footnotetext{
10 In the observers manuals for PACS and for SPIRE (see footnote 1), calibration errors are $3 \%, 5 \%, 10 \%, 10 \%$, and $10 \%$ at $70 \mu \mathrm{m}, 160 \mu \mathrm{m}$, $250 \mu \mathrm{m}, 350 \mu \mathrm{m}$, and $500 \mu \mathrm{m}$, respectively.
}

Table 2. Main physical properties of dense cores in NGC 6334.

\begin{tabular}{c|c|c|c}
\hline \hline \multicolumn{4}{c}{490 dense cores } \\
\hline & Min & Median & Max \\
\hline$F W H M_{\text {dec }}(\mathrm{pc})$ & 0.05 & $0.09 \pm 0.05$ & 0.3 \\
$\left\langle T_{\text {dust }}\right\rangle(\mathrm{K})$ & 9.5 & $20 \pm 6$ & 51 \\
$L_{\text {bol }}\left(L_{\odot}\right)$ & 4 & $860 \pm 6100$ & 87000 \\
Mass $\left(M_{\odot}\right)$ & 0.25 & $32 \pm 82$ & 1020 \\
$\left\langle n_{\mathrm{H}_{2}}\right\rangle\left(\mathrm{cm}^{-3}\right)$ & $1 \times 10^{4}$ & $(2 \pm 6) \times 10^{6}$ & $\geq 7 \times 10^{7}$ \\
\hline
\end{tabular}

\subsection{Luminosity and volume-averaged density}

We define the bolometric luminosity and volume-averaged density for our sample of dense cores. The bolometric luminosity, $L_{\text {bol }}$, should be calculated by integrating the flux densities over the source SED from $2 \mu \mathrm{m}$ to $1 \mathrm{~mm}$. To get an accurate bolometric luminosity for protostellar sources which can have significant mid-infrared emission, we used data from the GLIMPSE 3.6-8 $\mu \mathrm{m}$, MIPSGAL $24 \mu \mathrm{m}$, WISE $22 \mu \mathrm{m}$, and MSX $21 \mu \mathrm{m}$ catalogs ${ }^{11}$. These catalogs were cross-correlated with our MDCs within a radius of $6^{\prime \prime}$, roughly corresponding to the $160 \mu \mathrm{m}$ FWHM beam. When associations are found with both the MIPSGAL and WISE catalogs, or the MSX catalog, we favored that with the higher-resolution measurement. We estimated the midIR noise level in NGC 6334 as the mean dispersions in several background areas, $\sigma_{24 \mu \mathrm{m}}=5 \mathrm{mJy} / 6^{\prime \prime}$-beam.

Starless dense cores that do not show mid-infrared or even $70 \mu \mathrm{m}$ emission can theoretically have their luminosity calculated by integrating below their respective fitted modified blackbodies. Their bolometric luminosities are thus set to the maximum value of either this estimate or the one obtained by integrating below observed fluxes.

The volume-averaged density is taken as:

$\left\langle n_{H_{2}}\right\rangle=\frac{\text { Mass }}{\frac{4}{3} \pi \mu m_{\mathrm{H}}\left(F W H M_{\operatorname{Ref} \lambda}^{\mathrm{dec}} / 2\right)^{3}}$,

where $F W H M_{\operatorname{Ref} \lambda}^{\mathrm{dec}}$ is measured at the reference wavelength and deconvolved as explained in Sect. $4.1, \mu=2.8$ is the mean molecular weight (Kauffmann et al. 2008), and $m_{\mathrm{H}}$ is the hydrogen mass. For unresolved sources at $160 \mu \mathrm{m}$ or $250 \mu \mathrm{m}$, their upper limit size of 5.85" (resp. 9.1") leads to lower limits for their volume-averaged density defined by:

$\left\langle n_{\mathrm{H}_{2} \_\min }\right\rangle=\frac{\text { Mass }}{\frac{4}{3} \pi \mu m_{\mathrm{H}}\left(0.5 \times H P B W_{\operatorname{Ref} \lambda} / 2\right)^{3}}$.

Table 2 summarizes the main properties of our sample of 490 dense cores and Fig. 3 (top) displays their mass distribution.

\section{Complete sample of NGC 6334 MDCs}

The masses of dense cores extracted from Herschel data range from $0.3 M_{\odot}$ to approximately $1000 M_{\odot}$. If we expect high-mass stars to form in the most massive dense cores, we must establish the lowest mass required by a dense core to form at least one high-mass star.

\footnotetext{
11 The catalogs (GLIMPSE (I + II + 3D), MIPSGAL, WISE All-Sky Source, and MSXPSC v2.3) can be found via http://irsa.ipac. caltech.edu/applications/Gator/
} 

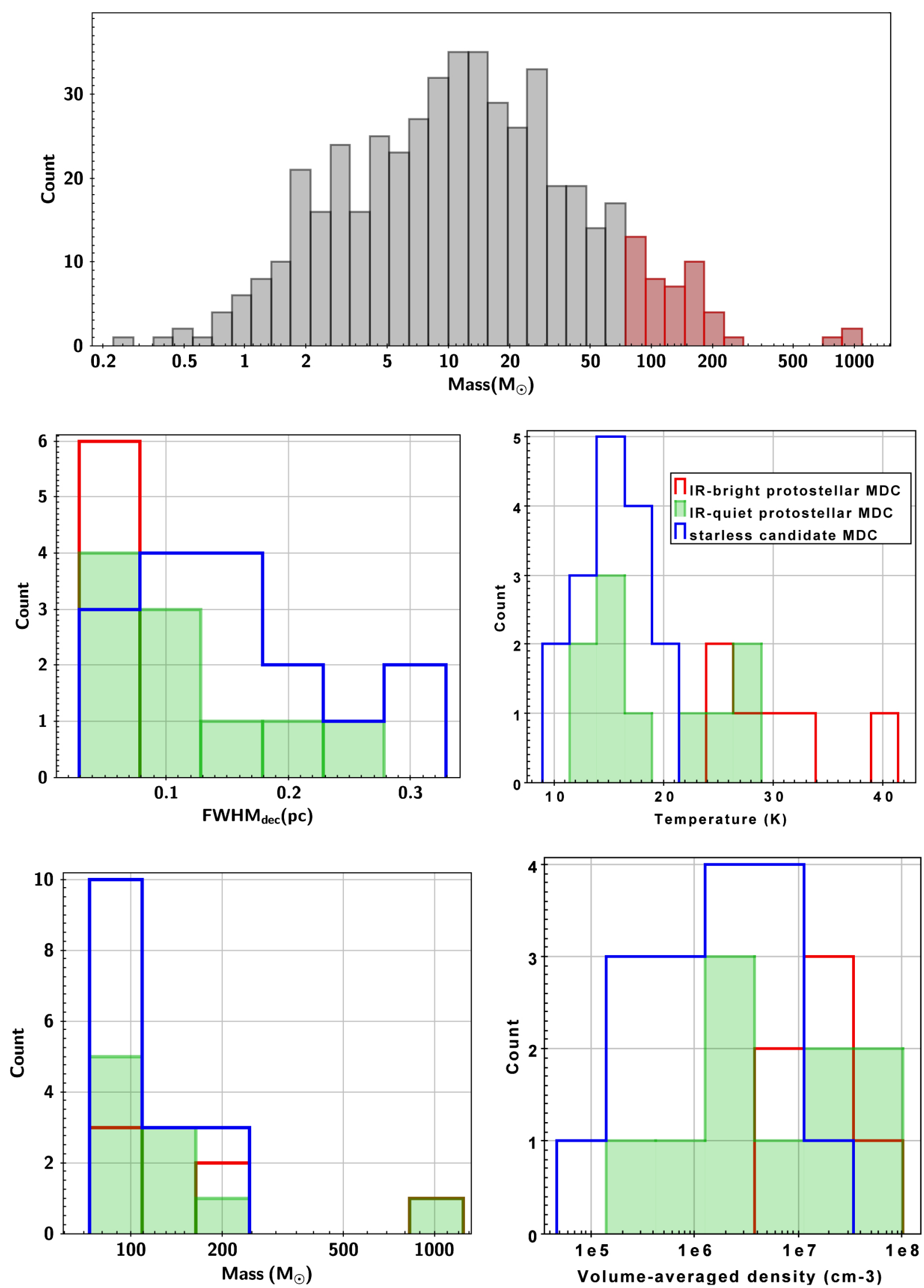

Fig. 3. Mass distribution of the 490 dense cores (gray histogram) and 46 MDCs (red histogram) of NGC 6334 (top). Sample is estimated to be complete down to $\sim 15 M_{\odot}$. Distributions of the deconvolved FWHM (middle-left), temperature (middle-right), mass (bottom-left), and volumeaveraged density (bottom-right) for the 32 reliable MDCs at various evolutionary phases (blue = starless candidate, green $=$ IR-quiet protostellar, red = IR-bright). MDCs become more massive, hotter, smaller, and thus denser along the high-mass star formation process. 


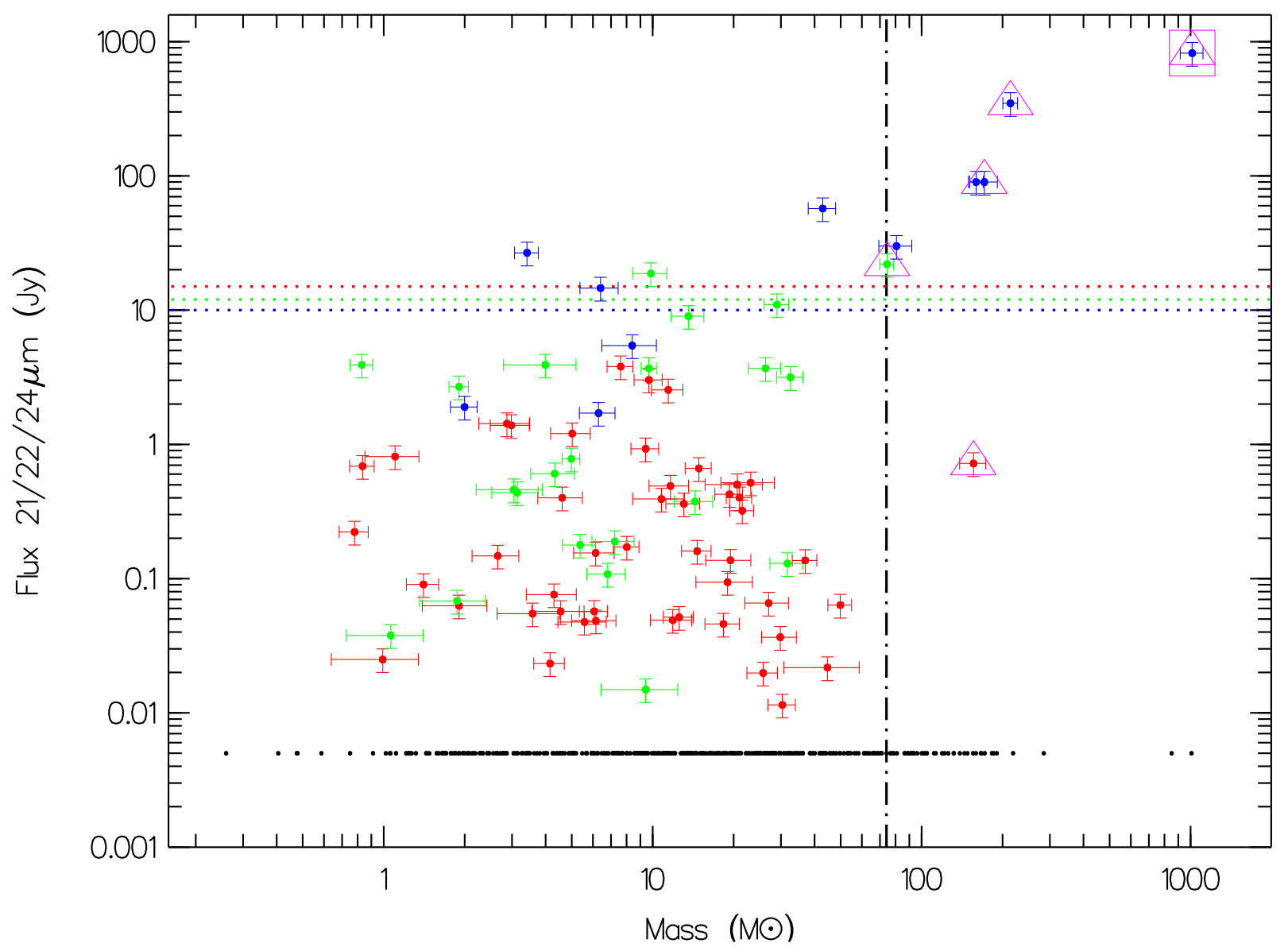

Fig. 4. Determination of the minimum mass for MDCs in NGC 6334 in a diagram displaying their $24 \mu \mathrm{m}$ (red), $22 \mu \mathrm{m}$ (green), or $21 \mu \mathrm{m}$ (blue) detected flux, or their $24 \mu \mathrm{m}$ (black) non-detection (set to the $1 \times \sigma_{24 \mu \mathrm{m}} \sim 5 \mathrm{mJy}$ point-source detection level; see Sect. 4.3) as a function of their mass. Pink squares and triangles denote dense cores associated with $20 \mathrm{~cm}$ compact radio sources and $\mathrm{CH}_{3} \mathrm{OH}$ masers, respectively. Red, green, and blue dotted lines are the $15 \mathrm{Jy}, 12 \mathrm{Jy}$, and $10 \mathrm{Jy}$ limits at $24 \mu \mathrm{m}, 22 \mu \mathrm{m}$, and $21 \mu \mathrm{m}$ for IR-bright sources, respectively. The black dotted-dashed line represents our mass threshold of $75 M_{\odot}$ for MDCs. Errors on the mass are taken from MPFIT while mid-infrared flux errors are estimated to be $20 \%$.

\subsection{MDCs with known high-mass star formation signposts}

For this purpose, we used two signposts of on-going highmass star formation: Class II $\mathrm{CH}_{3} \mathrm{OH}$ maser and compact radio centimeter sources. Masers result from the interaction of protostars with their envelope. In particular, $6.7 \mathrm{GHz}$ Class II $\mathrm{CH}_{3} \mathrm{OH}$ masers are found to be exclusively associated with highmass star formation (Breen et al. 2013). As for compact radio centimeter sources, they generally arise from ionizing UCH II regions. Dense cores displaying one of these signposts have already started to form high-mass stars. To pinpoint these highmass star-forming dense cores, we cross-correlated the positions of our sample of 490 dense cores with those of $6.7 \mathrm{GHz}$ Class II $\mathrm{CH}_{3} \mathrm{OH}$ masers having appropriate velocities and compact radio centimeter sources listed in published catalogs (e.g., White et al. 2005; Combi et al. 2011; Pestalozzi et al. 2005; Caswell et al. 2010).

Allowing up to $10^{\prime \prime}$ offsets, we found six associations. MDC \#6 is associated with both $6.7 \mathrm{GHz} \mathrm{CH}_{3} \mathrm{OH}$ maser emission (Caswell et al. 2010) and an UCH II region observed at $20 \mathrm{~cm}$ (White et al. 2005), while MDCs \#1 (source I), \#10, \#14 (source $\mathrm{I}(\mathrm{N})$ ), and \#46 are only associated with $6.7 \mathrm{GHz} \mathrm{CH}_{3} \mathrm{OH}$ maser sources (Pestalozzi et al. 2005; Caswell et al. 2010). In addition, we used $\mathrm{N}_{2} \mathrm{H}^{+}$observations published in Foster et al. (2011) and Russeil et al. (2010), and checked that dense cores indeed contain dense gas and that their velocities are close to the mean velocity measured for the NGC 6334 cloud complex of $-4 \mathrm{~km} \mathrm{~s}^{-1}$, with a velocity gradient (Kraemer \& Jackson 1999) from the center $\left(V_{\mathrm{LSR}} \sim 0 \mathrm{~km} \mathrm{~s}^{-1}\right)$ to the edge of the central filament $\left(V_{\mathrm{LSR}} \sim-11 \mathrm{~km} \mathrm{~s}^{-1}\right)$.

\subsection{Minimum mass for MDCs in NGC 6334}

To determine the minimum mass required for dense cores to form high-mass stars, we followed the method of Motte et al. (2007) and compared the dense cores of Table 2 with and without signposts of high-mass star formation.

Figure 4 shows the $24 \mu \mathrm{m}$ flux as a function of the mass of each source, unless $24 \mu \mathrm{m}$ flux is saturated, in which case the $22 \mu \mathrm{m}$ or $21 \mu \mathrm{m}$ flux is shown instead. The dense cores associated with $\mathrm{CH}_{3} \mathrm{OH}$ masers or compact radio centimeter sources are well separated from the others. Since those dense cores with on-going high-mass star formation exhibit 75-1000 $M_{\odot}$ masses, we fixed $75 M_{\odot}$ as the mass threshold for dense cores able to form high-mass stars in NGC 6334.

If one wants to compare the NGC 6334 mass threshold to that obtained in other studies, one must realize that the threshold depends on the typical size of the sources considered. For Cygnus X MDCs that have 30\% smaller mean sizes than NGC 6334 MDCs, Motte et al. (2007) found a mass limit of $40 M_{\odot}$ (see their Fig. 7), $45 \%$ lower than the one here. In 
contrast, Csengeri et al. (2014) used a much larger threshold, $>650 M_{\odot}$, to select, from the ATLASGAL survey, massive clumps with sizes of $0.4-1 \mathrm{pc}$, almost ten times larger than MDCs. The threshold found for NGC 6334 MDCs is also consistent with values used to distinguish intermediate-mass dense cores in three other HOBYS regions (Motte et al. 2010; Nguyen Luong et al. 2011; Fallscheer et al. 2013).

\subsection{MDC characteristics}

Using our lower mass limit of $75 M_{\odot}$ and keeping robust dense cores with reduced $\chi^{2}<10$, we ended up with a selection of 46 MDCs in NGC 6334. Table 3 lists each of these 46 MDCs along with their main physical properties as derived from the SED analysis of Sect. 4 and presented in Figs. B.1-B.46. As given in Table 4, MDCs have median FWHM sizes of $\sim 0.08 \mathrm{pc}$, dust temperatures of $\sim 17 \mathrm{~K}$, total masses around $120 M_{\odot}$, and median densities of $6 \times 10^{6} \mathrm{~cm}^{-3}$. Figure 3 displays their size, temperature, mass, and volume-averaged density distributions, with their mass distribution also shown on top of that of the complete sample of 490 dense cores. The role of MDCs in the highmass star formation scenario is to be the spatial and kinematical link between ridges or hubs and individual protostars.

For the most extended MDCs, we checked for infrared associations out to a radius of $11^{\prime \prime}$ from the peak. When a midinfrared counterpart was visible but had not been measured by earlier infrared surveys, we performed aperture photometry directly on the images using the GLIMPSE and MIPSGAL guidelines for point sources ${ }^{12}$. The resulting bolometric luminosities of NGC 6334 MDCs range from $10 L_{\odot}$ to $9 \times 10^{4} L_{\odot}$ (see Table 4 ).

We checked that there is a good agreement between the location of Herschel MDCs of Table 3 and the massive clumps extracted at $1.2 \mathrm{~mm}$ by Russeil et al. (2010). The MDCs found here are four times smaller in size and four times less massive than those clumps, that is, $\sim 0.1 \mathrm{pc}$ and $150 M_{\odot}$ versus $\sim 0.4 \mathrm{pc}$ and $600 M_{\odot}$, respectively. Most (8 out of 10 ) of the $1.2 \mathrm{~mm}$ clumps are subdivided into several MDCs in our data, showing that the present study allows us to follow the cloud-fragmentation process to smaller scales. The two $1.2 \mathrm{~mm}$ clumps which do not contain any Herschel MDCs are actually associated with compact $\mathrm{H}$ II regions. Hence, their dense cloud structures could already be ionized. The masses of these two clumps were also overestimated by Russeil et al. (2010) since they underestimated their dust temperature and free-free contribution to the $1.2 \mathrm{~mm}$ emission. MDCs of Table 3 are generally located within the $\geq 200 M_{\odot}$ clumps of Russeil et al. (2010), with the exception of a few that are found either close $(<0.3 \mathrm{pc})$ to them or within intermediatemass (100-200 $M_{\odot}$ ) clumps of Russeil et al. (2010). Therefore, the present sample of Herschel MDCs in NGC 6334 is consistent with the previous study done at a lower angular resolution and a single wavelength by Russeil et al. (2010).

\section{Discussion}

We now discuss the nature of the NGC 6334 MDCs (see Sect. 6.1) and use this sample to investigate the probability of the existence of high-mass prestellar cores (see Sect. 6.2), and

12 In GLIMPSE images, aperture, inner, and outer sky annulus radii are recommended to be $3.6^{\prime \prime}, 3.6^{\prime \prime}$, and 9.6" respectively (see https://irsa.ipac.caltech.edu/data/SPITZER/GLIMPSE/ doc/glimpse_photometry_v1.0.pdf). In MIPSGAL images, aperture, inner, and outer sky annulus radii are recommended to be 6.4", 7.6", and 17.8", respectively (Gutermuth \& Heyer 2015). give constraints on the high-mass star formation process (see Sects. 6.3, 6.4).

\subsection{Nature and evolutionary status of MDCs}

To define the nature of MDCs, we use their properties as derived from our extraction and SED fits as well as their appearance on Herschel and other submillimeter images (see Table 3 and Figs. B.1-B.46). Table 5 lists the 46 MDCs along with their SED properties and associated high-mass star formation signposts, which allow for determination of their probable nature. Figure 5 locates MDCs of all types (starless, IR-quiet, IR-bright, undefined) on the column density image of the NGC 6334 molecular complex.

\subsubsection{Undefined cloud structures, doubtful MDCs}

In principle, MDCs are local gravitational potential wells and should correspond to centrally concentrated cloud fragments, and thus far-infrared/(sub)millimeter emission peaks. getsources, the compact source extraction software we used (see Sect. 3.2), is powerful in deblending clusters of sources. It tends, however, to identify secondary sources around dominant ones. While some of these likely represent local gravitational wells, others may just be unbound cloud structures like the gas flows toward hubs/ridges. We therefore inspected, by eye, the column density, $160 \mu \mathrm{m}$, and ground-based (sub)millimeter images (see Figs. B.1-B.46) that have better angular resolution than the Herschel $250-500 \mu \mathrm{m}$ bands. From this inspection, we confirm 32 MDC candidates and identify 14 as not centrally peaked, undefined cloud structures. Submillimeter observations at even higher-angular resolutions toward some undefined cloud structures in particular confirm that they are indeed not centrally concentrated (André et al., in prep.).

The undefined cloud structures often have SED fits of poor quality with reduced $\chi^{2}>5$, suggesting they are rather diffuse. Furthermore, MDCs identified as undefined cloud structures are mostly located close to major column density peaks (see Fig. 5), whose intense far-infrared emission could have affected the source extraction. Despite our reservations, however, in the absence of a definite argument to remove these 14 undefined cloud structures from our sample, we keep them in Tables 5 and 3. Since their nature and evolutionary status are difficult to define, however, we exclude these undefined cloud structures in our following discussion.

\subsubsection{Separation between IR-quiet and IR-bright MDCs}

To determine the evolutionary statuses of the 32 remaining MDCs, we used the definition of IR-bright versus IR-quiet protostellar MDCs of Motte et al. (2007), followed by Russeil et al. (2010) and Nguyen Luong et al. (2011). Based on the predicted mid-infrared emission of a B3-type stellar embryo, the minimum fluxes of IR-bright MDCs located at $1.75 \mathrm{kpc}$ should be $S_{21 \mu \mathrm{m}}=10 \mathrm{Jy}$ (Motte et al. 2010), $S_{22 \mu \mathrm{m}}=12 \mathrm{Jy}$, and $S_{24 \mu \mathrm{m}}=$ 15 Jy (Russeil et al. 2010). With these criteria, IR-bright MDCs should already host at least one stellar embryo of mass larger than $8 M_{\odot}$ while IR-quiet MDCs only contain stellar embryos of lower mass, which could further accrete. IR-quiet MDCs could therefore evolve into IR-bright MDCs of similar masses. For context, the HMPOs of Beuther et al. (2002) are IR-bright cloud structures ranging from $1 \mathrm{pc}$ clumps to $0.01 \mathrm{pc}$ cores, among which are several tens of IR-bright MDCs. In Fig. 4, we separate 
Table 3. Physical parameters of the 46 MDCs found in NGC 6334

\begin{tabular}{|c|c|c|c|c|c|c|}
\hline $\begin{array}{c}\text { Number } \\
\text { Id. }\end{array}$ & $\begin{array}{c}F W H M_{\mathrm{dec}} \\
(\mathrm{pc})\end{array}$ & $\begin{array}{c}\left\langle T_{\text {dust }}\right\rangle \\
(\mathrm{K})\end{array}$ & $\begin{array}{l}\text { Mass } \\
\left(M_{\odot}\right) \\
\end{array}$ & $\begin{array}{l}L_{\text {bol }} \\
\left(L_{\odot}\right) \\
\end{array}$ & $\begin{array}{c}\left\langle n_{\mathrm{H}_{2}}\right\rangle \\
\left(\mathrm{cm}^{-3}\right)\end{array}$ & $\chi^{2}$ \\
\hline \multicolumn{7}{|c|}{ IR-bright protostellar MDC } \\
\hline $\begin{array}{c}1 \\
6 \\
10 \\
32 \\
38 \\
46 \\
\end{array}$ & $\begin{array}{l}\leq 0.08 \\
\leq 0.08 \\
\leq 0.08 \\
\leq 0.08 \\
\leq 0.08 \\
\leq 0.05\end{array}$ & $\begin{array}{l}24.6 \pm 1.1 \\
40.1 \pm 0.7 \\
27.3 \pm 1.5 \\
30.8 \pm 2.0 \\
33.1 \pm 2.3 \\
25.9 \pm 0.3 \\
\end{array}$ & $\begin{array}{c}1020 \pm 100 \\
200 \pm 10 \\
180 \pm 20 \\
95 \pm 10 \\
90 \pm 10 \\
75 \pm 4 \\
\end{array}$ & $\begin{array}{c}42600 \\
87200 \\
9600 \\
8300 \\
9300 \\
3200 \\
\end{array}$ & $\begin{array}{l}\geq 7.4 \times 10^{7} \\
\geq 1.5 \times 10^{7} \\
\geq 1.3 \times 10^{7} \\
\geq 6.8 \times 10^{6} \\
\geq 6.4 \times 10^{6} \\
\geq 2.0 \times 10^{7}\end{array}$ & $\begin{array}{l}3 \\
2 \\
1 \\
2 \\
3 \\
2\end{array}$ \\
\hline \multicolumn{7}{|c|}{ IR-quiet protostellar MDC } \\
\hline $\begin{array}{c}2 \\
7 \\
14 \\
15 \\
24 \\
30 \\
33 \\
37 \\
41 \\
42 \\
\end{array}$ & $\begin{array}{c}\leq 0.08 \\
\leq 0.08 \\
\leq 0.05 \\
0.11 \\
0.18 \\
0.13 \\
0.11 \\
0.13 \\
\leq 0.05 \\
0.26 \\
\end{array}$ & $\begin{array}{l}15.2 \pm 0.4 \\
27.2 \pm 1.5 \\
26.6 \pm 1.4 \\
23.5 \pm 1.2 \\
16.9 \pm 0.5 \\
14.4 \pm 0.4 \\
12.8 \pm 0.5 \\
11.7 \pm 0.6 \\
26.2 \pm 1.4 \\
14.0 \pm 0.4\end{array}$ & $\begin{array}{c}950 \pm 80 \\
200 \pm 20 \\
160 \pm 15 \\
160 \pm 20 \\
110 \pm 10 \\
100 \pm 10 \\
90 \pm 10 \\
90 \pm 15 \\
85 \pm 10 \\
80 \pm 10 \\
\end{array}$ & $\begin{array}{c}940 \\
6400 \\
7000 \\
2200 \\
580 \\
70 \\
30 \\
28 \\
2200 \\
50 \\
\end{array}$ & $\begin{array}{c}\geq 7.0 \times 10^{7} \\
\geq 1.4 \times 10^{7} \\
\geq 4.3 \times 10^{7} \\
4.3 \times 10^{6} \\
6.0 \times 10^{5} \\
1.6 \times 10^{6} \\
2.0 \times 10^{6} \\
1.3 \times 10^{6} \\
\geq 2.3 \times 10^{7} \\
1.6 \times 10^{5} \\
\end{array}$ & $\begin{array}{c}6 \\
0.4 \\
5 \\
8 \\
3 \\
3 \\
5 \\
5 \\
1 \\
3 \\
\end{array}$ \\
\hline \multicolumn{7}{|c|}{ Starless MDC candidate } \\
\hline \begin{tabular}{|c|}
5 \\
11 \\
13 \\
16 \\
17 \\
21 \\
26 \\
27 \\
28 \\
31 \\
35 \\
36 \\
39 \\
40 \\
44 \\
45 \\
\end{tabular} & $\begin{array}{c}0.08 \\
0.28 \\
0.18 \\
0.15 \\
0.1 \\
0.27 \\
\leq 0.08 \\
0.14 \\
\leq 0.08 \\
0.17 \\
0.21 \\
0.08 \\
0.19 \\
0.12 \\
0.3 \\
0.11 \\
\end{array}$ & $\begin{array}{c}14.6 \pm 0.4 \\
14.1 \pm 0.6 \\
9.5 \pm 0.4 \\
9.9 \pm 0.4 \\
16.5 \pm 0.7 \\
18.3 \pm 0.6 \\
21.1 \pm 2.4 \\
15.6 \pm 0.6 \\
19.2 \pm 2.2 \\
11.9 \pm 0.4 \\
16.4 \pm 0.9 \\
17.7 \pm 0.7 \\
12.1 \pm 0.4 \\
18.1 \pm 0.8 \\
12.8 \pm 0.6 \\
14.7 \pm 0.8 \\
\end{array}$ & $\begin{array}{c}210 \pm 15 \\
170 \pm 20 \\
170 \pm 30 \\
150 \pm 20 \\
150 \pm 15 \\
130 \pm 10 \\
100 \pm 30 \\
105 \pm 11 \\
100 \pm 24 \\
100 \pm 14 \\
90 \pm 10 \\
90 \pm 9 \\
85 \pm 15 \\
85 \pm 10 \\
77 \pm 10 \\
76 \pm 10 \\
\end{array}$ & $\begin{array}{c}160 \\
110 \\
10 \\
11 \\
230 \\
390 \\
740 \\
120 \\
410 \\
22 \\
140 \\
220 \\
20 \\
240 \\
27 \\
60 \\
\end{array}$ & $\begin{array}{c}\geq 1.5 \times 10^{7} \\
2.4 \times 10^{5} \\
1.0 \times 10^{6} \\
1.6 \times 10^{6} \\
4.8 \times 10^{6} \\
2.2 \times 10^{5} \\
\geq 7.5 \times 10^{6} \\
1.3 \times 10^{6} \\
\geq 7.3 \times 10^{6} \\
6.2 \times 10^{5} \\
3.4 \times 10^{5} \\
5.7 \times 10^{6} \\
4.4 \times 10^{5} \\
1.7 \times 10^{6} \\
1.0 \times 10^{5} \\
2.0 \times 10^{6} \\
\end{array}$ & $\begin{array}{c}3 \\
3 \\
8 \\
10 \\
4 \\
6 \\
6 \\
6 \\
7 \\
4 \\
4 \\
6 \\
9 \\
10 \\
3 \\
4 \\
\end{array}$ \\
\hline \multicolumn{7}{|c|}{ Undefined cloud structure } \\
\hline $\begin{array}{c}3 \\
4 \\
8 \\
9 \\
12 \\
18 \\
19 \\
20 \\
22 \\
23 \\
25 \\
29 \\
34 \\
43 \\
\end{array}$ & $\begin{array}{l}\leq 0.08 \\
\leq 0.08 \\
\leq 0.08 \\
\leq 0.08 \\
\leq 0.08 \\
0.26 \\
\leq 0.08 \\
\leq 0.08 \\
\leq 0.08 \\
\leq 0.08 \\
\leq 0.08 \\
0.09 \\
0.11 \\
\leq 0.08\end{array}$ & $\begin{array}{l}14.7 \pm 0.4 \\
15.4 \pm 1.0 \\
15.3 \pm 1.1 \\
19.2 \pm 0.7 \\
24.1 \pm 1.1 \\
11.5 \pm 0.5 \\
17.7 \pm 1.1 \\
19.4 \pm 0.8 \\
15.8 \pm 0.9 \\
21.9 \pm 1.1 \\
21.5 \pm 0.9 \\
22.8 \pm 0.4 \\
11.9 \pm 0.8 \\
15.2 \pm 0.6\end{array}$ & $\begin{array}{l}820 \pm 70 \\
280 \pm 40 \\
180 \pm 35 \\
180 \pm 20 \\
170 \pm 20 \\
140 \pm 20 \\
140 \pm 20 \\
130 \pm 15 \\
125 \pm 20 \\
120 \pm 15 \\
110 \pm 10 \\
100 \pm 10 \\
90 \pm 25 \\
80 \pm 10 \\
\end{array}$ & $\begin{array}{c}670 \\
300 \\
190 \\
720 \\
2600 \\
50 \\
340 \\
540 \\
160 \\
1100 \\
870 \\
3300 \\
20 \\
80 \\
\end{array}$ & $\begin{array}{c}\geq 5.9 \times 10^{7} \\
\geq 2.0 \times 10^{7} \\
\geq 1.3 \times 10^{7} \\
\geq 1.3 \times 10^{7} \\
\geq 1.2 \times 10^{7} \\
2.6 \times 10^{5} \\
\geq 9.9 \times 10^{6} \\
\geq 9.2 \times 10^{6} \\
\geq 9.0 \times 10^{6} \\
\geq 8.7 \times 10^{6} \\
\geq 8.0 \times 10^{6} \\
4.5 \times 10^{6} \\
2.3 \times 10^{6} \\
\geq 5.9 \times 10^{6}\end{array}$ & $\begin{array}{c}8 \\
4 \\
9 \\
1 \\
3 \\
3 \\
5 \\
4 \\
5 \\
3 \\
4 \\
7 \\
10 \\
7 \\
\end{array}$ \\
\hline
\end{tabular}


Table 4. Main physical properties of MDCs in NGC 6334.

\begin{tabular}{|c|c|c|c|}
\hline \multicolumn{4}{|c|}{46 MDCs } \\
\hline & Min & Median & Max \\
\hline$F W H M_{\mathrm{dec}}(\mathrm{pc})$ & 0.05 & 0.08 & 0.3 \\
\hline$\left\langle T_{\text {dust }}\right\rangle(\mathrm{K})$ & 9.5 & 16.7 & 40.1 \\
\hline$L_{\mathrm{bol}}\left(L_{\odot}\right)$ & 10 & 320 & 87000 \\
\hline $\operatorname{Mass}\left(M_{\odot}\right)$ & 75 & 120 & 1020 \\
\hline$\left\langle n_{\mathrm{H}_{2}}\right\rangle\left(\mathrm{cm}^{-3}\right)$ & $1 \times 10^{5}$ & $6 \times 10^{6}$ & $\geq 7 \times 10^{7}$ \\
\hline
\end{tabular}

IR-bright and IR-quiet MDCs using the mid-infrared flux limits given above. The Spitzer $24 \mu \mathrm{m}$ fluxes were primarily used but, in saturated areas, the $22 \mu \mathrm{m}$ WISE or $21 \mu \mathrm{m} M S X$ fluxes were the alternative choices (see Table A.8). Among MDCs of Table 5, six qualify as IR-bright protostellar MDCs: MDCs \#1, $\# 6$, \#10, \#32, \#38, and \#46, as seen in Fig. 4. Four of these are also associated with $\mathrm{CH}_{3} \mathrm{OH}$ maser sources (Pestalozzi et al. 2005).

Given their spatial sizes $(\sim 0.1 \mathrm{pc})$, MDCs cannot be precursors of single high-mass stars. MDCs with weak or even undetected mid-infrared emission probably host young protostars and/or prestellar cores. MDCs are qualified as "IR-quiet protostellar" if they are associated with compact emission at $70 \mu \mathrm{m}$. Most of these, however, are also detected at mid-infrared (21 $\mu \mathrm{m}$ to $24 \mu \mathrm{m})$ wavelengths. Similarly, a detection at both 21-24 $\mu \mathrm{m}$ and $70 \mu \mathrm{m}$ mitigates the misidentification of externally heated diffuse cores without internal stellar embryos as being IR-quiet MDCs. "Starless" MDC candidates are generally sources for which no compact emission at $70 \mu \mathrm{m}$ emission can be distinguished above the noise level of Herschel images. In a few cases, however, they have weak $70 \mu \mathrm{m}$ emission at the level of or below that of the SED fits to longer far-infrared and submillimeter wavelengths (see, e.g., Figs. B.17-B.32, also Motte et al. 2010).

For each MDC, we compared the ratio of the submillimeter luminosity to the bolometric luminosity, $L_{\mathrm{bol}}$, for the IRquiet versus IR-bright subsamples. The submillimeter luminosity, $L_{\text {submm }}=L_{\geq 350 \mu \mathrm{m}}$, is defined as the integrated luminosity below the resulting SED fit between $350 \mu \mathrm{m}(857 \mathrm{GHz})$ and $1200 \mu \mathrm{m}(250 \mathrm{GHz})$. The submillimeter to bolometric luminosity ratio has previously been used in the low-mass regime to distinguish between Class 0 and Class I protostars. André et al. (2000) found a separation in this ratio amongst their individual protostars (size $\sim 0.01-0.1 \mathrm{pc}$ ) with a luminosity ratio less than $1 \%$ for Class I protostars. We cannot, however, directly use the same threshold values taken to separate Class 0 and Class I protostars. Indeed, the physical sizes, stellar luminosities, and cloud environments of MDCs and low-mass protostars are different enough to justify a change of this ratio. In the intermediate- to high-mass regime, Hennemann et al. (2010) and Nguyen Luong et al. (2011) found a clear separation between IR-bright and IR-quiet MDCs in terms of $L_{\geq 350 \mu \mathrm{m}} / L_{\mathrm{bol}}$ ratio. Namely, IR-bright MDCs or UCH II regions have small $(<1 \%)$ ratios and IR-quiet or starless MDCs can have ratios ranging from $1 \%$ to $20 \%$.

Figure 6 displays the submillimeter to bolometric luminosity ratio of all NGC 6334 dense cores as a function of their mass. In this diagram, IR-bright MDCs, as defined above, are well separated from IR-quiet MDCs and lower-mass dense cores. They exhibit high masses $\left(M \geq 75 M_{\odot}\right)$ and low submillimeter to bolometric luminosity ratios $\left(L_{\text {submm }} / L_{\text {bol }}<0.5-1 \%\right)$. MDCs with $L_{\text {submm }} / L_{\text {bol }}$ ratios larger than $1 \%$ with $\beta=2$ or 1.5 , are all IR-quiet. Among the five MDCs with $L_{\text {submm }} / L_{\text {bol }}=0.5-1 \%$, the one associated with compact infrared emission at $8 \mu \mathrm{m}$ and $22 \mu \mathrm{m}, \mathrm{MDC} \# 46$, is an IR-bright protostellar MDC while the others are IR-quiet protostellar MDCs. NGC 6334-I(N) or MDC $\# 14$ itself has a $L_{\mathrm{submm}} / L_{\mathrm{bol}}=0.5 \%$ ratio but its mid-infrared emission is small enough for it to be an IR-quiet protostellar MDC, in agreement with the Sandell (2000) and Brogan et al. (2009) classifications of this object. Therefore, we confirm that there is a good but not perfect agreement between evolutionary classifications made with mid-infrared fluxes and $L_{\text {submm }} / L_{\text {bol }}$ ratios.

The present sample of MDCs can help us evaluate the probability that high-mass prestellar cores exist in NGC 6334. Below, we first investigate if the starless MDCs of NGC 6334 could host high-mass prestellar cores (see Sect. 6.2) and then discuss the lifetime of any prestellar phase (see Sect. 6.3).

\subsection{Quest for high-mass prestellar cores in MDCs}

Our Herschel study has identified a new population of MDCs; 16 starless candidates (see Table 5). The present MDC sample can help us evaluate the probability that high-mass prestellar cores do exist in NGC 6334, especially within its starless MDCs. Thanks to our careful SED analysis using multi-wavelength Herschel images, the number of starless MDC candidates is much larger than found using the SIMBA $1 \mathrm{~mm}$ image only by Russeil et al. (2010), that is, 16 to 1 . The dust temperatures of starless MDCs have been measured to be $\sim 15 \mathrm{~K}$ (from $9.5 \mathrm{~K}$ to $21.1 \mathrm{~K}$, see Fig. 3 middle-right), almost five degrees lower than the $20 \mathrm{~K}$ uniform temperature assumed by Russeil et al. (2010). With $20 \mathrm{~K}$ instead of $15 \mathrm{~K}$, mass estimates from a flux measurement at $1 \mathrm{~mm}$ are underestimated by $\sim 30 \%$. This difference alone can explain why the number of starless MDC candidates more massive than $75 M_{\odot}$ was largely underestimated before Herschel. Indeed, if we were to suppose a uniform temperature of $20 \mathrm{~K}$ for each MDC, only one starless candidate, MDC \#26, would be considered massive enough. Figure 7 locates the starless MDC candidates and protostellar MDCs of NGC 6334 on a mass versus size diagram, where the gas mass concentration of other protostellar and starless samples is displayed (Bontemps et al. 2010; Butler \& Tan 2012; Tan et al. 2013; Duarte-Cabral et al. 2014; Wang et al. 2014). An empirical relation close to Mass $(<r) \propto r^{2}$ is found to link starless clumps/MDCs and their prestellar core candidates. With a fluxscaling correction consistent with this relation, the number of starless MDCs in NGC 6334 reduces to 13, showing that 16 is definitively an upper limit for the number of starless MDCs in NGC 6334.

That said, the natures of the 16 starless MDC candidates of Table 5 remain in question. Nine are characterized by poorly defined SEDs (with $5<\chi^{2}<10$, see Col. 7 of Table 3 ), casting doubts on the quality of their flux extraction and mass estimates, and thus their nature. Moreover, all MDCs with poor fits (except MDC \#21) are located against concentrated filamentary structures (see, e.g., Figs. B.24-B.32), explaining why fluxes extracted from the highest-resolution images deviate from those obtained from their SED fits, largely set by Herschel fluxes at moderate-angular resolution. Such complex structures cast doubt on the ability of at least half of the $\sim 0.1 \mathrm{pc}$ starless MDC candidates to concentrate enough mass into a $\sim 0.02 \mathrm{pc}$ high-mass prestellar core. Of note, the location of MDC \#17, close to a dominant column density source, suggests it could instead be a gas flow structure attracted by the dominant source rather 
Table 5. 46 MDCs: Nature and additional comments of $>75 M_{\odot}$ compact sources: 32 MDCs and 14 undefined cloud structures.

\begin{tabular}{|c|c|c|c|c|c|}
\hline $\begin{array}{c}\text { Number } \\
\text { Id }\end{array}$ & Nature & Comments & $\begin{array}{c}8 \mu \mathrm{m} \\
\text { source }\end{array}$ & $\begin{array}{c}21-24 \mu \mathrm{m} \\
\text { flux }\end{array}$ & $\begin{array}{c}L_{\text {submm }} / L_{\text {bol }} \\
(\%)\end{array}$ \\
\hline 1 & & UCH II region NGC 6334- $\mathrm{F}^{1}$. NGC6334- $\mathrm{I}^{2} . \mathrm{CH}_{3} \mathrm{OH}$ Maser ${ }^{3}$. & Y & Strong & 0.4 \\
\hline 6 & IR-bright & NGC 6334-V². EGO. $\mathrm{CH}_{3} \mathrm{OH}$ Maser $^{2}$. & Y & Strong & 0.1 \\
\hline 10 & & Close to $\mathrm{CH}$ II region NGC6334-C ${ }^{1} . \mathrm{CH}_{3} \mathrm{OH}$ Maser $^{3}$. & Y & Strong & 0.4 \\
\hline 32 & protostellar & Partially contains \#38. & $\mathrm{Y}$ & Strong & 0.3 \\
\hline 38 & MDC & NGC6334-IV (A) ${ }^{4}$ & $\mathrm{Y}$ & Strong & 0.2 \\
\hline 46 & & NGC634-IV $(\mathrm{MM} 3)^{5}$. $\mathrm{CH}_{3} \mathrm{OH} \mathrm{Maser}^{3}$. & $\mathrm{Y}$ & Strong & 0.5 \\
\hline 2 & & Poor fit. Not centrally peaked. Partially contains \#14. & $\mathrm{N}$ & None & 6.5 \\
\hline 7 & & Close to \#6. & $\mathrm{N}$ & None & 0.6 \\
\hline 14 & & Poor fit. NGC6334-I(N) ${ }^{6} . \mathrm{CH}_{3} \mathrm{OH} \mathrm{Maser}^{3}$. & $\mathrm{N}$ & Weak & 0.5 \\
\hline 15 & IR-quiet & Poor fit. Not centrally peaked. Close to \#6. & $\mathrm{N}$ & None & 1.2 \\
\hline 24 & protostollor. & & $\mathrm{Y}$ & Weak & 1.6 \\
\hline 30 & protostellar & & $\mathrm{N}$ & None & 7.9 \\
\hline 33 & MDC & Poor fit. & $\mathrm{N}$ & None & 11.7 \\
\hline 37 & & Poor fit. & $\mathrm{Y}$ & Weak & 10.1 \\
\hline 41 & & & $\mathrm{~N}$ & None & 0.8 \\
\hline 42 & & & $\mathrm{~N}$ & None & 8.5 \\
\hline $5^{* *}$ & & & $\mathrm{~N}$ & None & 7.4 \\
\hline $11^{*}$ & & & $\mathrm{~N}$ & None & 8.5 \\
\hline 13 & & Poor fit. & $\mathrm{N}$ & None & 27.7 \\
\hline 16 & & Poor fit. & $\mathrm{N}$ & None & 24.8 \\
\hline 17 & & Close to \#6. & $\mathrm{N}$ & None & 4.8 \\
\hline $21^{*}$ & & Poor fit. Extended $160 \mu \mathrm{m}$. & $\mathrm{N}$ & None & 3.2 \\
\hline 26 & starless & Poor fit. May contain background emission. & $\mathrm{N}$ & None & 1.8 \\
\hline 27 & & Poor fit. May contain background emission. & $\mathrm{N}$ & None & 5.8 \\
\hline 28 & $\mathrm{MDC}$ & Poor fit. May contain background emission. & $\mathrm{N}$ & None & 2.7 \\
\hline $31^{*}$ & candidate & & $\mathrm{N}$ & None & 14.5 \\
\hline $35^{*}$ & & & $\mathrm{~N}$ & None & 4.9 \\
\hline 36 & & Poor fit. & $\mathrm{N}$ & None & 3.7 \\
\hline 39 & & Poor fit. Missing submm measures & $\mathrm{N}$ & None & 13.9 \\
\hline 40 & & Poor fit. May contain background emission. & $\mathrm{N}$ & None & 3.4 \\
\hline $44^{*}$ & & & $\mathrm{~N}$ & None & 11.6 \\
\hline $45^{*}$ & & May contain background emission. & $\mathrm{N}$ & None & 7.3 \\
\hline 3 & & Poor fit. Not centrally peaked. Close to \#14. & $\mathrm{N}$ & None & 7.2 \\
\hline 4 & & Not centrally peaked. Close to \#14. & $\mathrm{N}$ & None & 6.2 \\
\hline 8 & & Poor fit. Not centrally peaked. & $\mathrm{N}$ & None & 6.2 \\
\hline 9 & & Not centrally peaked. Close to \#46. & $\mathrm{N}$ & None & 2.7 \\
\hline 12 & & Not centrally peaked. & $\mathrm{N}$ & None & 1.1 \\
\hline 18 & undefined & Overlap with IR source. Not centrally peaked. & $\mathrm{Y}$ & Weak & 9.2 \\
\hline 19 & lond & Poor fit. Not centrally peaked. Close to \#46. & $\mathrm{N}$ & None & 3.7 \\
\hline 20 & cloud & Not centrally peaked. Partially contains \#46. & $\mathrm{N}$ & None & 2.6 \\
\hline 22 & structure & Not centrally peaked. Close to H II region GM1-247. & $\mathrm{N}$ & None & 5.6 \\
\hline 23 & & Not centrally peaked. Close to H II region GM1-247. & $\mathrm{N}$ & None & 1.6 \\
\hline 25 & & Not centrally peaked. Close to \#32. & $\mathrm{N}$ & None & 1.7 \\
\hline 29 & & Poor fit. Not centrally peaked. Close to \#6. & Y & None & 0.5 \\
\hline 34 & & Poor fit. Not centrally peaked. Missing submm measures. & $\mathrm{N}$ & None & 14.7 \\
\hline 43 & & Poor fit. Not centrally peaked. & $\mathrm{N}$ & None & 6.5 \\
\hline
\end{tabular}

Notes. "Poor" means fit with reduced $5<\chi^{2}<10$. "Strong" and "Weak" means $21-24 \mu \mathrm{m}$ flux respectively greater or lower than the minimum flux of IR-bright MDCs defined in Sect. 6.1.2. ${ }^{(*)}$ Is for MDCs among the best starless candidates.

References. (1) Rodriguez et al. (1982). (2) Loughran et al. (1986). (3) Pestalozzi et al. (2005) and Caswell et al. (2010). (4) Persi \& Tapia (2010). (5) Tapia et al. (2009). (6) Gezari (1982). (7) Tapia et al. (1991).

than a centrally-concentrated starless MDC hosting a high-mass prestellar core.

Visually speaking, the best starless MDC candidates could be MDCs \#21 (though it has a poor fit), \#44, \#35, \#11, and \#39 since they are the most isolated MDCs, with the least filamentary structures in their background. Their masses range from $80 M_{\odot}$ to $170 M_{\odot}$ but they have among the largest deconvolved sizes of our sample, $0.2-0.3 \mathrm{pc}$ (see Col. 2 of Table 3) like in the Rosette molecular cloud (Motte et al. 2010). Their extracted characteristics lead to densities of $1-5 \times 10^{5} \mathrm{~cm}^{-3}$, more typical of lowmass prestellar cores than MDCs $\left(10^{5} \mathrm{~cm}^{-3}\right.$ versus $10^{6} \mathrm{~cm}^{-3}$, see Ward-Thompson et al. 1999; Motte et al. 2007). In contrast, 


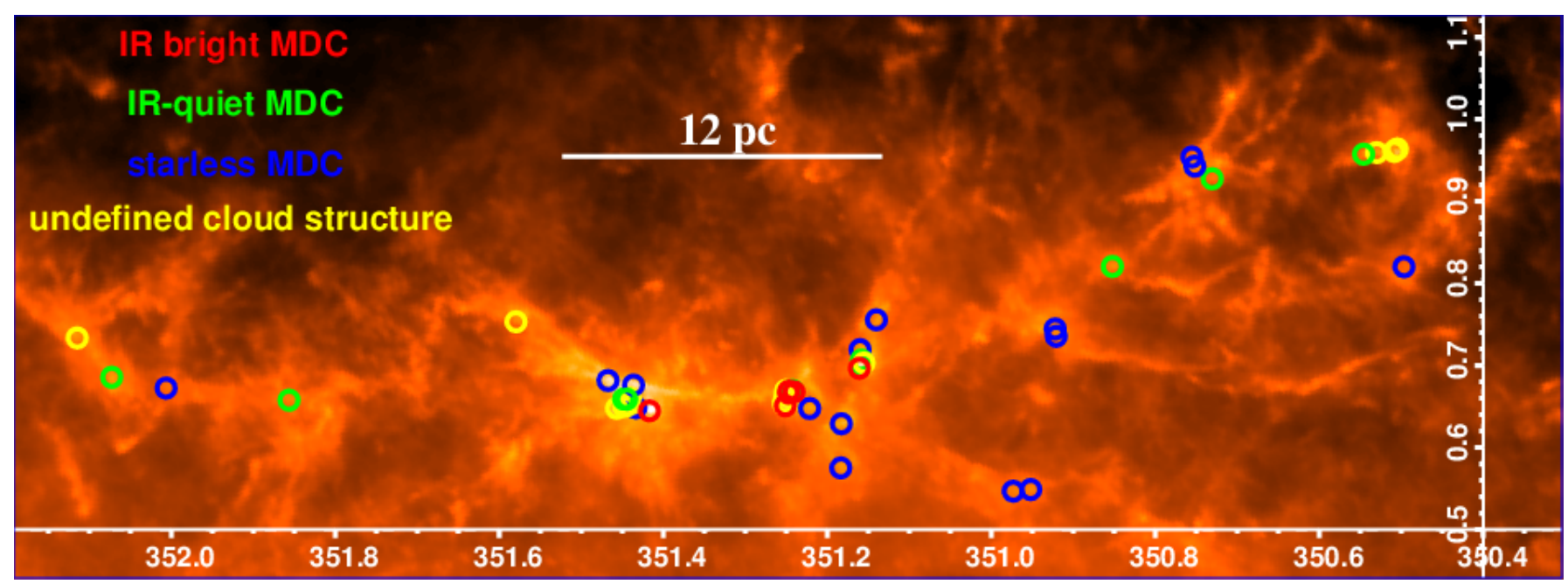

Fig. 5. Evolutionary subsamples of MDCs in NGC 6334, as defined in Sect. 6.1, plotted on the high-resolution $\left(\mathrm{HPBW} \sim 18.2^{\prime \prime}\right) N_{\mathrm{H}_{2}}$ columndensity image of the molecular complex. IR-bright MDCs cluster in the central part of the region whereas IR-quiet protostellar MDCs and starless MDC candidates are more widely distributed.

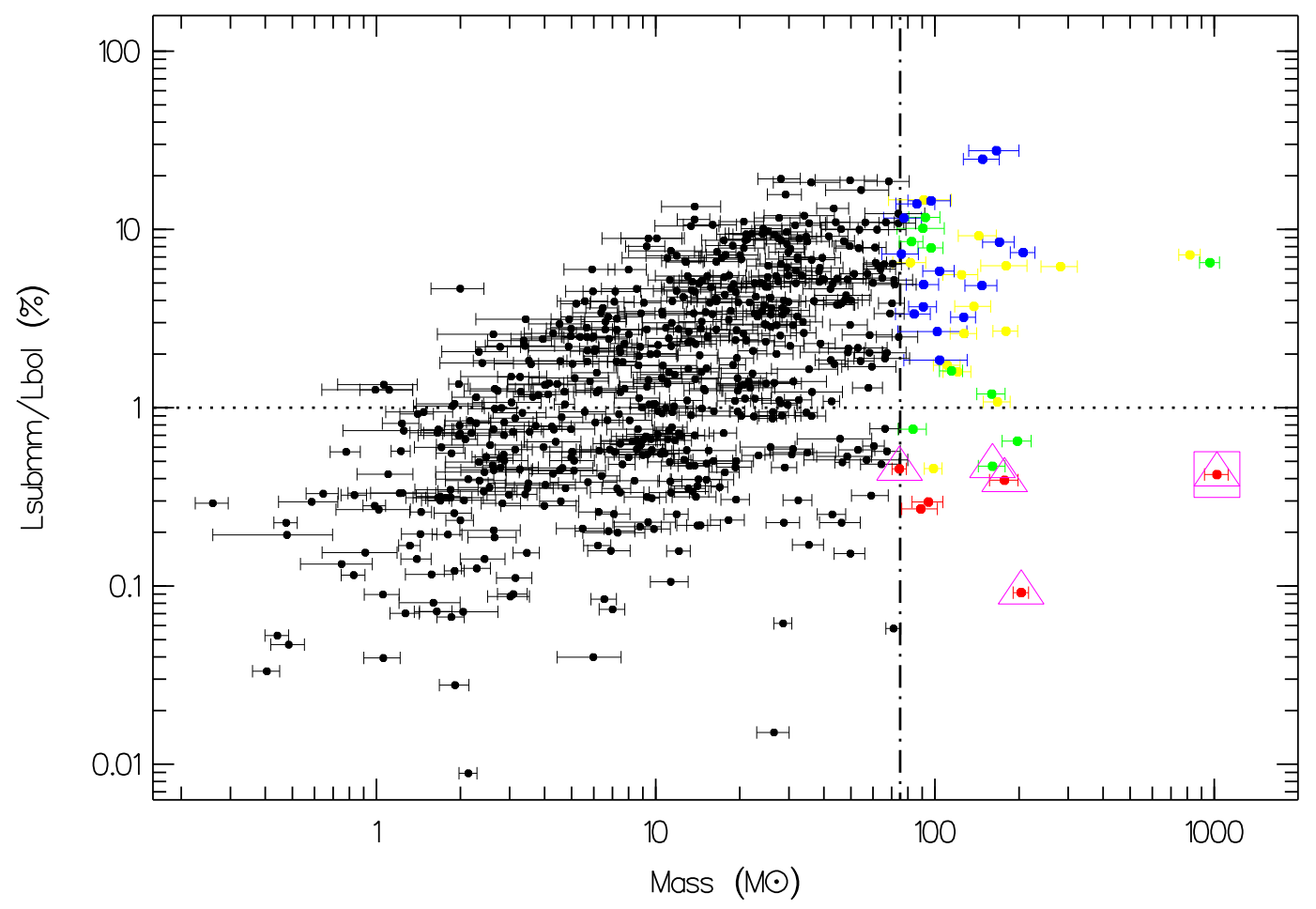

Fig. 6. $L_{\text {submm }} / L_{\text {bol }}$ versus mass diagram of NGC 6334 dense cores (black dots). Pink squares and triangles denote MDCs associated with radio centimeter compact and $\mathrm{CH}_{3} \mathrm{OH}$ maser sources, respectively. Red/green/blue/yellow dots highlight IR-bright MDCs, IR-quiet MDCs, starless MDC candidates, and undefined cloud structures, respectively (as defined in Sect. 6.1). Mass errors are given by MPFIT.

mass and density-wise $\left(150-210 M_{\odot}, n_{\mathrm{H} 2}>5 \times 10^{6} \mathrm{~cm}^{-3}\right)$, MDCs \#5, \#13, \#16, and \#17 would be the best starless MDC candidates. As mentioned above however, MDCs \#13 and \#16 have poorly defined SEDs and MDC \#17 may not be centrally concentrated enough. In the end, MDC \#5 is our best candidate starless MDC with its unresolved size, $\leq 0.08 \mathrm{pc}$, and high mass, $\sim 210 M_{\odot}$. Interestingly, these characteristics make it at least five times denser and thus more favorable to host a high-mass prestellar core than the starless MDC candidate of Tan et al. (2013).

To explore even further the probability of NGC 6334 MDCs containing high-mass prestellar cores or high-mass protostars, we used the mass versus size diagram of Fig. 7. The masses and FWHMs of NGC 6334 MDCs are compared with those of two $\sim 0.1 \mathrm{pc}$ MDC samples for which the $\sim 0.02 \mathrm{pc}$-scale content is known (see Bontemps et al. 2010; Tan et al. 2013). The gas mass concentration of the six most massive Cygnus $\mathrm{X}$ MDCs follows a classical Mass $(<r) \propto r$ relation, using masses and sizes estimated by Motte et al. (2007) and Bontemps et al. (2010). This relation suggests these MDCs have a centrally concentrated density distribution of $\rho(r) \propto r^{-2}$ or steeper, similar to those of MDCs or hubs on $\sim 0.1-1$ pc scales (e.g., Beuther et al. 2002; Didelon et al. 2015) or low-mass protostellar envelopes on 0.01-0.1 pc scales (e.g., Motte \& André 2001). By contrast, the starless structures found by submillimeter surveys or within 


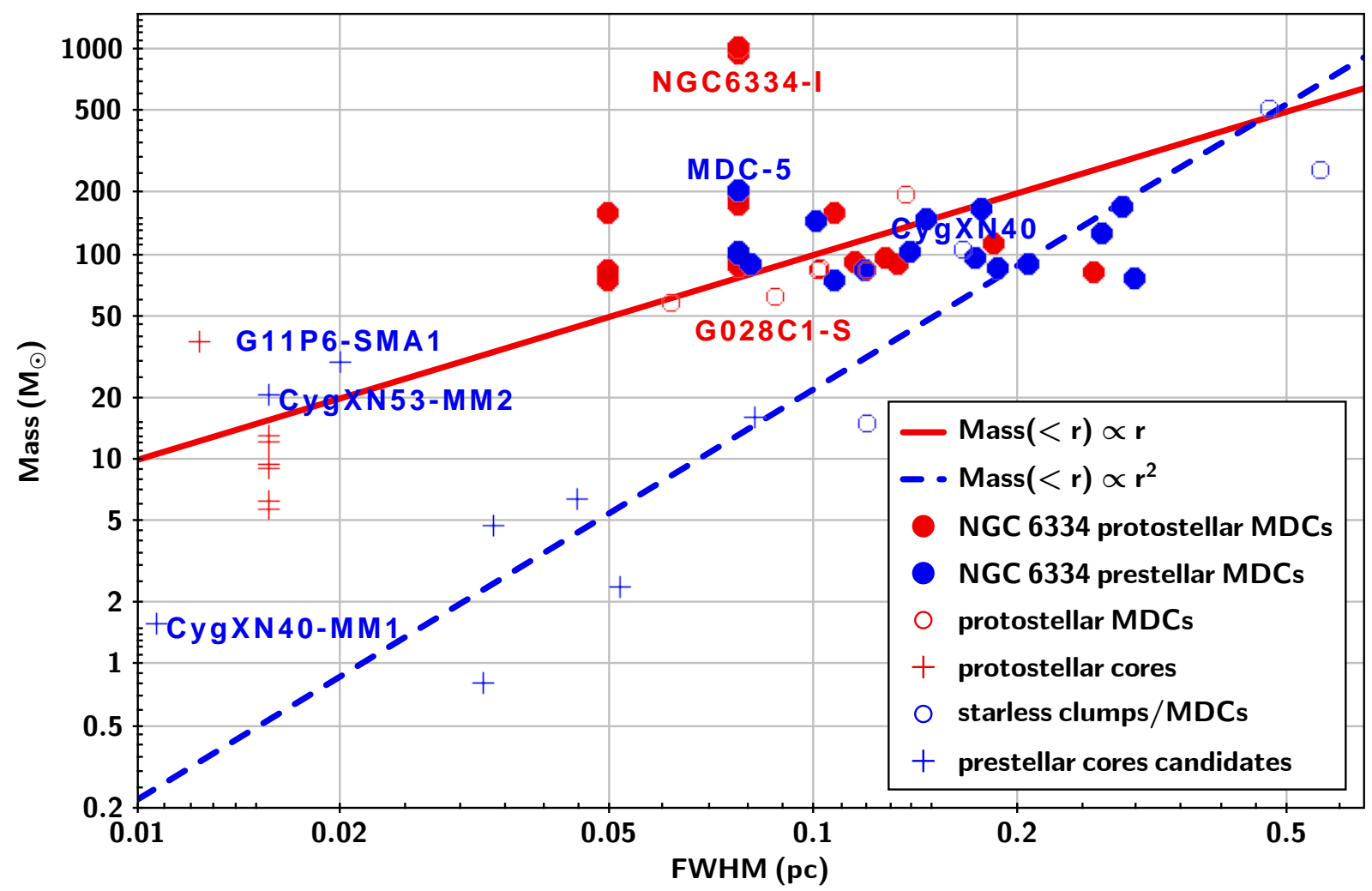

Fig. 7. Predicting gas concentration within NGC 6334 MDCs through a mass versus size diagram. Starless MDC candidates in NGC 6334, displayed in filled blue circles, are compared with a handful of massive starless clumps, and prestellar sub-fragments/cores (Bontemps et al. 2010; Butler \& Tan 2012; Tan et al. 2013; Duarte-Cabral et al. 2014; Wang et al. 2014, in blue open circles and crosses), respectively. NGC 6334 protostellar MDCs, displayed in filled red circles, are themselves compared to five protostellar MDCs and their embedded high-mass protostars (Motte et al. 2007; Bontemps et al. 2010; Tan et al. 2016, in red open circles and crosses), respectively. The red line and blue dashed line represent mass radial power-laws of Mass $(<r) \propto r$ and Mass $(<r) \propto r^{2}$, expected to be followed by NGC 6334 protostellar and starless MDCs respectively. The sample of $32 \mathrm{MDCs}$ is too small to present any statistically meaningful mass versus size relation.

infrared dark clouds are much less concentrated and tend to follow a $\operatorname{Mass}(<r) \propto r^{2}$ relation (Butler \& Tan 2012; Tan et al. 2013). Note that the only known starless MDC N40 in Cygnus$\mathrm{X}$ follows a similar relation (see Bontemps et al. 2010, Fig. 7). Given their low density $\left(1-3 \times 10^{5} \mathrm{~cm}^{-3}\right)$, these MDC fragments at $0.02 \mathrm{pc}$ may better represent intermediate- to low-mass prestellar cores. Most starless MDC candidates in NGC 6334 are in fact 1-10 times denser than IRDC fragments (see Fig. 3 bottom-right). If they follow a $\operatorname{Mass}(<r) \propto r^{2}$ relation, they could on average form $0.02 \mathrm{pc}$ cores, each of $1-5 M_{\odot}$ mass. Given that eight starless MDCs already display non-centrally peaked morphologies, and that the seven remaining MDCs are not all very dense, the maximum number of high-mass prestellar cores expected in NGC 6334 would be seven. If MDC \#5 is the only good starless MDC of NGC 6334, the minimum number of high-mass prestellar cores could be one. An ALMA project targeting the starless MDCs of NGC 6334 should soon reveal if they do indeed host groups of intermediate- to low-mass prestellar cores or simply a few dominant high-mass prestellar cores.

\subsection{Constraints on the high-mass star formation process}

At the chosen $0.1 \mathrm{pc}$ size, our large sample of 490 dense cores should be complete for $>15 M_{\odot}$ cloud structures, according to completeness simulations performed for getsources extractions in similar Herschel images (e.g., Könyves et al. 2015; Marsh et al. 2016) and the dense core mass distribution shown in Fig. 3 top. Therefore, the present sample must be complete for the $>75 M_{\odot}$ MDCs hosting massive young stellar objects and allows us to start building an evolutionary scenario for the formation of high-mass stars. While starless MDCs cannot all form high-mass stars (see Sect. 6.2), IR-quiet MDCs will probably become IR-bright and form high-mass stars. Indeed, IR-quiet MDCs generally have more powerful $\mathrm{SiO}$ outflows and stronger mid-IR emission than a cluster of low-mass Class 0s (Motte et al. 2007; Russeil et al. 2010).

Table 6 lists the median characteristics of each MDC evolutionary phase and provides estimates of their statistical lifetime. With the relative number of IR-bright and IR-quiet protostellar MDCs as well as starless MDC candidates with respect to OB stars in the NGC 6334 molecular complex, we can infer the statistical lifetimes of each evolutionary phase. We use the work of Russeil et al. (2013), who performed a statistical census of O-B3 ionizing stars in the NGC 6334-NGC 6357 complex. They estimated a total number of $\sim 150$ high-mass stars in NGC 6334, which encompasses $\sim 2 / 3$ of the NGC 6334-6357 gas mass $\left(\sim 7 \times 10^{5} M_{\odot}\right)$. We assume the median age of the 150 O-B3 stars of NGC 6334 to be $1.5 \pm 0.5 \times 10^{6}$ yr according to the age of the main clusters identified by Kharchenko et al. (2013), Morales et al. (2013), and Getman et al. (2014). We also use the fragmentation level of Cygnus X protostellar MDCs (Bontemps et al. 2010) and assumed that IR-quiet and IR-bright protostellar MDCs in NGC 6334 should host, on average, two high-mass protostars. As for starless MDC candidates, given our discussion in Sect. 6.2, we assume that only one to seven of them could host at most one high-mass prestellar core. 
Table 6. Median properties and lifetime estimates for MDCs of different evolutionary states.

\begin{tabular}{|c|c|c|c|c|}
\hline & IR-bright MDC & IR-quiet MDC & starless MDC candidate & O-B3 stars \\
\hline Number & 6 & 10 & 16 & 150 \\
\hline Size $(\mathrm{pc})$ & 0.08 & 0.11 & 0.14 & - \\
\hline$\left\langle T_{\text {dust }}\right\rangle(\mathrm{K})$ & 29 & 16 & 15 & - \\
\hline$L_{\mathrm{bol}}\left(L_{\odot}\right)$ & 9500 & 760 & 130 & - \\
\hline $\operatorname{Mass}\left(M_{\odot}\right)$ & 140 & 106 & 103 & - \\
\hline$\left\langle n_{\mathrm{H}_{2}}\right\rangle\left(\mathrm{cm}^{-3}\right)$ & $1.4 \times 10^{7}$ & $3.1 \times 10^{6}$ & $1.4 \times 10^{6}$ & - \\
\hline Free-fall time ${ }^{1}$ & $9 \times 10^{3} \mathrm{yr}$ & $2 \times 10^{4} \mathrm{yr}$ & $3 \times 10^{4} \mathrm{yr}$ & - \\
\hline $\begin{array}{l}\text { Number of high-mass cores } \\
\text { Statistical lifetime of high-mass cores }\end{array}$ & \multicolumn{2}{|c|}{$\begin{array}{l}32 \text { high-mass protostars } \\
\qquad 3 \times 10^{5} \mathrm{yr}\end{array}$} & $\begin{array}{l}\text { 1-7 high-mass prestellar cores } \\
\qquad 1-7 \times 10^{4} \mathrm{yr}\end{array}$ & $1.5 \times 10^{6} \mathrm{yr}$ \\
\hline
\end{tabular}

Notes. ${ }^{(1)}$ Free-fall time measured from the median values of the density averaged over the full MDC volume, which is approximately a sphere with a FWHM radius: $\left\langle n_{\mathrm{H}_{2}}\right\rangle_{\text {full }}=\left\langle n_{\mathrm{H}_{2}}\right\rangle / 8$ and $t_{\text {free-fall }}=\sqrt{\frac{3 \pi}{32 G \mu m_{\mathrm{H}}<n_{\mathrm{H}_{2}}>\text { full }}}$. ${ }^{\text {(2) }}$ We assumed that protostellar MDCs host $\sim 2$ protostars as was found for Cygnus-X by Bontemps et al. (2010), while 1 to 7 starless MDCs could host one high-mass prestellar core. ${ }^{(3)}$ The statistical lifetimes of high-mass protostars/prestellar cores is estimated from the relative numbers of high-mass protostars/prestellar cores versus OB stars.

Accordingly, we obtain lifetimes of $1.1 \times 10^{5} \mathrm{yr}, 1.9 \times 10^{5} \mathrm{yr}$, and $1-7 \times 10^{4} \mathrm{yr}$, for the IR-bright, IR-quiet, and prestellar phases of high-mass stars in NGC 6334, respectively. The complete duration of the protostellar phase, $3 \times 10^{5} \mathrm{yr}$, is only twice that of previous estimates ${ }^{13}\left(\sim 1.5 \times 10^{5} \mathrm{yr}\right.$ according to Motte et al. 2007; Russeil et al. 2010). The main difference is the fragmentation level here taken to be 2 while Motte et al. (2007) and Russeil et al. (2010) assumed that each MDC would form a single high-mass progenitor. The complete duration of the protostellar core phase corresponds to a few free-fall times of MDCs with $\sim 10^{6} \mathrm{~cm}^{-3}$ densities averaged over twice their FWHM and a single free-fall time of their $\sim 10^{5} \mathrm{~cm}^{-3}$ parental clump (Sect. 3.1). The lifetime of the prestellar core phase itself measured to be $1-7 \times 10^{4} \mathrm{yr}$ could either be as short as a single free-fall time or even be nonexistent.

Other constraints arise with the new population of 16 starless MDC candidates, which we found to account for $50 \%$ of the complete sample of MDCs. This proportion is much larger than in previous MDC studies (e.g. Motte et al. 2007; Russeil et al. 2010, 2\% ${ }^{14}$ and 6\%). As stated in Sect. 6.2, however, many starless MDCs listed in Table 3 may not be able to host high-mass prestellar cores. The short statistical lifetime of starless MDCs is discussed by Motte et al. (2007), who suggest that high-mass prestellar cores could be dynamically evolving into protostars.

Recent studies showed that the formation of ridges and massive stellar clusters are dynamical events, which are tightly linked (e.g., Nguyen-Luong et al. 2013; Louvet et al. 2016). Indeed, both ridges and hubs are observed to form through a global free-fall collapse (e.g., Schneider et al. 2010b; Hill et al. 2011; Hennemann et al. 2012), followed by intense formation of high-mass stars quoted as mini-starbursts (e.g., Motte et al. 2003; Nguyen Luong et al. 2011; Louvet et al. 2014). The shape and velocity drift of the south-western part of the ridge (i.e., the connecting filament in Fig. 2, see also Zernickel et al. 2013; André et al. 2016) recalls sub-filaments falling onto ridges, like those around DR21 (Schneider et al. 2010b). Interestingly, the

13 The statistical lifetime of protostellar MDCs has been corrected by Russeil et al. (2010) from values given in Motte et al. (2007).

14 The SiO emission used by Motte et al. (2007) to classify Cyg X-N40 as a protostellar MDC has since been largely if not totally associated to cloud-cloud collision by Duarte-Cabral et al. (2014). Cyg X-N40 thus is the only starless MDC candidate in Cygnus X. lack of MDCs within the south-western sub-filament suggests it is stretched while falling onto the gravitation potential of the NGC 6334 ridge (see MDC distribution in Fig. 5). Moreover, a burst of star formation appears to have occurred recently in the central part of NGC 6334, corresponding to its ridge (Willis et al. 2013). In the context of the kinematical studies of ridges and hubs, the lifetime estimates of the present paper imply that either high-mass prestellar cores form and evolve in a single free-fall time of ridges/hubs or that their masses continue increasing well within the protostellar phase. The prestellar phase of high-mass star formation may be a starless MDC containing a few low-mass prestellar cores, that subsequently evolves into an IR-quiet protostellar MDC hosting a low-mass protostellar core.

The main physical properties (size, temperature, and mass) of the different evolutionary subsamples exhibit clear differences, as shown in Table 6 . The median deconvolved size decreases by a factor of two during MDC evolution from starless candidate to IR-quiet and finally IR-bright MDC. While this increasing compactness suggests that MDCs continue contracting, gathering material as they evolve, it also reflects our improved ability to detect cold starless MDCs at longer wavelengths with lower angular resolution. We confirmed this decreasing size trend by comparing the $250 \mu \mathrm{m}$ deconvolved sizes of all MDCs, and IR-bright protostellar MDCs. The average temperatures themselves rise from $\sim 15 \mathrm{~K}$ to $\sim 29 \mathrm{~K}$, from starless or IR-quiet protostellar to IR-bright protostellar MDCs. Protostellar MDCs also seem to have masses increasing by $30 \%$ from their IR-quiet to their IR-bright phases. More surprisingly, starless MDCs are on average a factor of four smaller in density than protostellar MDCs: $\sim 1.4 \times 10^{5} \mathrm{~cm}^{-3}$ versus $\sim 5 \times 10^{6} \mathrm{~cm}^{-3}$ (see also Fig. 3), respectively. Either protostars grow in stellar mass at the same time as their MDC gas reservoir condenses or starless MDC candidates identified here do not have the ability to form high-mass stars in the near future.

The spatial distribution of the different evolutionary subsamples is shown on the high-resolution column density map in Fig. 5. MDCs are distributed along massive filaments and especially at their junctions, where material may accumulate (e.g., Schneider et al. 2012). IR-bright MDCs are found at the very center of the region, within the NGC 6334 ridge and hubs (see Sect. 3.1 and Fig. 2), suggesting, once again, that filaments and protostars grow in mass simultaneously. 

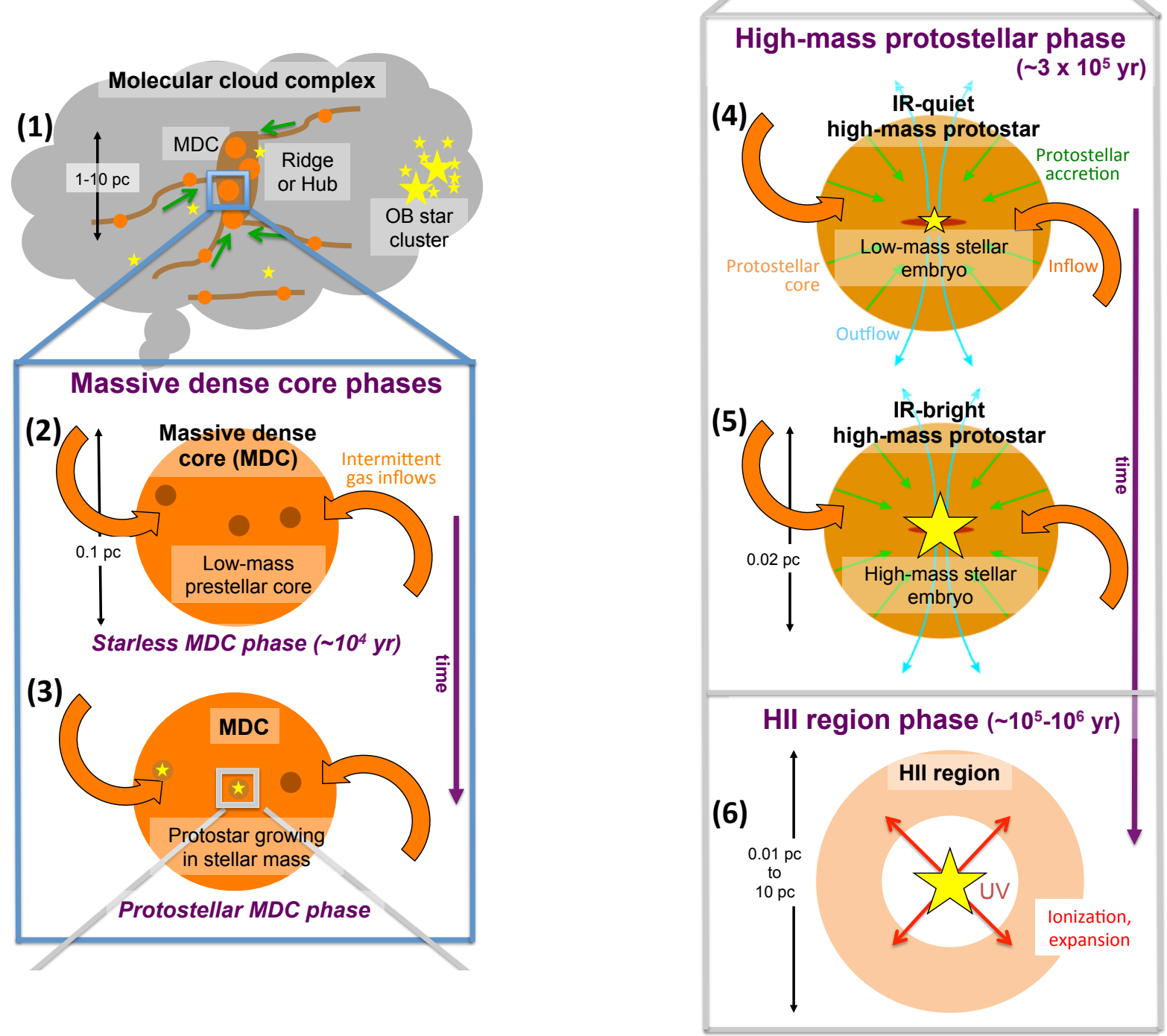

Fig. 8. Schematic evolutionary diagram proposed for the formation of high-mass stars. (1) Massive filaments and spherical clumps, called ridges and hubs, host massive dense cores (MDCs, $0.1 \mathrm{pc}$ ) forming high-mass stars. (2) During their starless phase, MDCs only harbor low-mass prestellar cores. (3) IR-quiet MDCs become protostellar when hosting a stellar embryo of low mass. The local 0.02 pc protostellar collapse is accompanied by the global 0.1-1 pc collapse of MDCs and ridges/hubs. (4) Protostellar envelopes feed from these gravitationally-driven inflows, leading to the formation of high-mass protostars. The latter are IR-quiet as long as their stellar embryos remain low-mass. (5) High-mass protostars become IRbright for stellar embryos with masses larger than $8 M_{\odot}$. (6) The main accretion phase terminates when the stellar UV field ionizes the protostellar envelope and an $\mathrm{H}$ II region develops.

\subsection{Proposed scenario for the formation of high-mass stars}

Combining the statistical constraints of the present paper on MDCs with results on ridges/hubs leads to a scenario for the formation of high-mass stars, which is sketched in Fig. 8 (see also Motte et al. 2017). While the association between the later $\mathrm{H}$ II region phase and gas ionization by the nascent $\mathrm{OB}$ star UV field is rather well known (Churchwell 2002; Zavagno et al. 2007, see Fig. 8, the 6th scheme), the earliest phases have only recently been unveiled.

Ridges and hubs represent the first and largest-scale phase of high-mass star formation (see Fig. 8, 1st scheme). One of the main results of the Herschel-HOBYS survey has been the recognition of ridges and hubs as hyper-massive clumps preferentially forming high-mass stars (e.g., Hill et al. 2011; Nguyen Luong et al. 2011, see also present paper). Schneider et al. (2010b), Peretto et al. (2013), and Didelon et al. (2015), among others, showed that gas was continuously inflowing onto ridges or hubs, probably like the northern part of the connecting filament toward the NGC 6334 ridge (see Fig. 2).

Ridges and hubs play a major role in driving gas flows and matter concentration from parsec scales down to the $0.1 \mathrm{pc}$ and finally $0.01 \mathrm{pc}$ scales of MDCs and protostellar cores (see Fig. 8, 2nd-3rd and 4th-5th schemes). While ridges form rich clusters of high-mass stars (e.g., Hill et al. 2011; Hennemann et al. 2012; Nguyen-Luong et al. 2013), hubs may just form a couple of high-mass stars (e.g., Peretto et al. 2013; Didelon et al. 2015). These structures may represent the parsec-scale reservoir, from which gas is accreted onto 0.02 pc-scale cores.

In such a dynamical scenario, ridges/hubs, MDCs, protostellar envelopes, and stellar embryos would most likely form simultaneously. A high-mass prestellar phase may therefore not be necessary for high-mass star formation. The present study demonstrates a possible lack of high-mass prestellar cores, with the largest statistics and the most accurate mass estimates so far obtained. 
At this stage, the most probable process to form high-mass stars thus is associated with converging flows and does not go through any high-mass prestellar core phase (see Fig. 8). Therefore during their starless phase, MDCs could only host a couple of prestellar cores of low to intermediate mass (2nd scheme). These prestellar cores should shortly become protostars, embedded within protostellar MDCs (3rd scheme). Protostars qualify as high-mass and IR-quiet if they are embedded within MDCs (>75 $M_{\odot}$ within $0.1 \mathrm{pc}$ ) but their stellar embryos remain lowmass, $<8 M_{\odot}$ (4th scheme). When large amounts of inflowing gas reach these low-mass protostars, they could become high-mass IR-bright protostars with a $>8 M_{\odot}$ stellar embryo (5th scheme), $\mathrm{H}$ II regions (6th scheme), and finally O-B3 type stars.

\section{Conclusions}

To improve our knowledge of the high-mass star formation process, we performed an unbiased study of its earliest phases in the entire NGC 6334 molecular cloud complex. As part of the Herschel-HOBYS key program, we especially aimed at finding MDCs hosting the high-mass analogs of low-mass prestellar cores and Class 0 protostars. We used Herschel far-infrared space-based and submillimeter images together with midinfrared and (sub)millimeter ground-based data (see Table 1) to obtain a complete census of $32 \mathrm{MDCs}$, which are the most likely progenitors of high-mass stars at $0.1 \mathrm{pc}$ scales. Our main findings can be summarized as follows:

1. The Herschel imaging of NGC 6334 gives a complete view of the cloud structures ranging from $0.05 \mathrm{pc}$ to $30 \mathrm{pc}$, from dense cores to clouds (see Figs. 1, and C.6-C.8). In the column density image, we outlined the NGC 6334 ridge and hubs, which are $\sim 10^{5} \mathrm{~cm}^{-3}$-density, 1 pc clumps, believed to form high-mass stars (see Fig. 2).

2. The exhaustive dataset gathered for the well-known NGC 6334 complex has been used as a template to determine the necessary analysis steps of HOBYS studies, focused on high-mass young stellar objects. We used getsources, an extraction software dedicated to multi-wavelength datasets. getsources performed a multi-resolution analysis of $\mathrm{Her}$ schel and complementary images, extracted 4733 compact sources, and provided their multi-wavelength fluxes.

3. We established a detailed procedure, for which including (sub)millimeter fluxes and scaling Herschel/SPIRE fluxes to $160 \mu \mathrm{m}$ or $250 \mu \mathrm{m}$ FWHM sizes are crucial steps. We visually inspected all mid-infrared to millimeter images independently and carefully constrained the complete SED of each compact source (see, e.g., Figs. B.1-B.46). Using criteria developed to identify the precursors of high-mass stars, we ensured extracted sources are genuine dense cloud structures. The resulting sample contains 490 well-characterized dense cores with typical FWHM sizes of $\sim 0.1$ pc (see Table 2).

4. We cross-correlated the NGC 6334 dense core sample with catalogs of compact radio centimeter and $\mathrm{CH}_{3} \mathrm{OH}$ maser sources, which are signposts of high-mass star formation. We determined a lower mass limit of $75 M_{\odot}$ for high-mass star formation to occur in the NGC 6334 region and listed the multi-wavelength fluxes and physical characteristics of 32 NGC 6334 MDCs and 14 undefined cloud structures (see Tables 5-3 and Tables A.2-A.12). MDCs have temperatures ranging from $9.5 \mathrm{~K}$ to $40 \mathrm{~K}$, masses from $75 M_{\odot}$ to $1000 M_{\odot}$, and volume-averaged densities from $1 \times 10^{5} \mathrm{~cm}^{-3}$ to $7 \times 10^{7} \mathrm{~cm}^{-3}$ (see Table 4 and Fig. 3 ).
5. We used mid-infrared catalogs and images to expand the SEDs of the MDCs beyond far-infrared to millimeter fluxes. Integrating MDC fluxes from $3.6 \mu \mathrm{m}$ to $1200 \mu \mathrm{m}$ leads to bolometric luminosities ranging from $10 L_{\odot}$ to $9 \times 10^{4} L_{\odot}$ (see Table 4). We separated the MDCs into IR-bright (evolved) and IR-quiet (young) protostellar using the flux of their compact counterparts at 21-24 $\mu \mathrm{m}$ (see Fig. 4). This evolutionary discriminator is consistent with a submillimeter-tobolometric luminosity ratio limit of $L_{\text {submm }} / L_{\text {bol }} \sim 0.5-1 \%$ (see Fig. 6). Starless MDC candidates themselves are cold cloud fragments which are associated with at most a weak and compact $70 \mu \mathrm{m}$ emission, which falls on the SED fits to their $\geq 160 \mu$ m fluxes.

6. Using Herschel and complementary (sub)millimeter data, we were able to constrain properly SEDs with cold temperatures. As a consequence, the present study allowed the discovery of 16 starless MDC candidates (see Figs. B.1-B.32). The nature of these MDCs must be further investigated. For various reasons, we doubt their ability to concentrate gas to high density and thus form high-mass stars in the nearfuture (see Fig. 7). The high-mass prestellar core phase thus remains elusive and may even not exist.

7. We used the respective proportion of MDCs found in the different evolutionary subsamples (6 IR-bright, 10 IR-quiet, and 16 starless) to infer their statistical lifetimes. Assumptions on their sub-fragmentation level lead to protostellar and prestellar lifetimes for high-mass star formation of $3 \times 10^{5} \mathrm{yr}$ and 1-7 $\times 10^{4} \mathrm{yr}$, respectively. We also found a clear increase in temperature and density in MDCs from the starless phase to the IR-quiet and IR-bright phases, suggesting that while heating its parental MDC gas, the protostellar embryo, and its parental MDC, grow in mass simultaneously (see Table 6). During star formation, MDCs tend to become more compact and cluster in denser parts of NGC 6334 (see Fig. 5), underlining the link between the formation of ridges/hubs and high-mass stars.

8. The present study shows that high-mass prestellar cores are still to be found. This lack of good candidates agrees with previous cloud structure analyses made by, for example, HOBYS. It favors a scenario wherein ridges/hubs, MDCs, and high-mass protostellar embryos form simultaneously, as shown in Fig. 8.

Acknowledgements. We are grateful to Alexander Men'shchikov for his help and discussions on getsources. SPIRE has been developed by a consortium of institutes led by Cardiff Univ. (UK) and including Univ. Lethbridge (Canada); NAOC (China); CEA, LAM (France); IFSI, Univ. Padua (Italy); IAC (Spain); Stockholm Observatory (Sweden); Imperial College London, RAL, UCL-MSSL, UKATC, Univ. Sussex (UK); and Caltech, JPL, NHSC, Univ. Colorado (USA). This development has been supported by national funding agencies: CSA (Canada); NAOC (China); CEA, CNES, CNRS (France); ASI (Italy); MCINN (Spain); SNSB (Sweden); STFC, UKSA (UK); and NASA (USA). PACS has been developed by a consortium of institutes led by MPE (Germany) and including UVIE (Austria); KU Leuven, CSL, IMEC (Belgium); CEA, LAM (France); MPIA (Germany); INAF-IFSI/OAA/OAP/OAT, LENS, SISSA (Italy); IAC (Spain). This development has been supported by the funding agencies BMVIT (Austria), ESA-PRODEX (Belgium), CEA/CNES (France), DLR (Germany), ASI/INAF (Italy), and CICYT/MCYT (Spain). This research has made use of the SIMBAD database, operated at CDS, Strasbourg, France. Part of this work was supported by the ANR (Agence Nationale pour la Recherche) project "PROBeS", number ANR-08-BLAN-0241. We acknowledge financial support from "Programme National de Physique Stellaire" (PNPS) and program "Physique et Chime du Milieu Interstellaire" (PCMI) of CNRS/INSU, France. G.J.W. gratefully acknowledges the receipt of a Leverhulme Emeritus Professorial Fellowship. 


\section{References}

André, P., Ward-Thompson, D., \& Barsony, M. 2000, Protostars and Planets IV,

André, P., Revéret, V., Könyves, V., et al. 2016, A\&A, 592, A54 Benjamin, R. A., Churchwell, E., Babler, B. L., et al. 2003, PASP, 115, 953

Bernard, J.-P., Paradis, D., Marshall, D. J., et al. 2010, A\&A, 518, L88

Beuther, H., \& Schilke, P. 2004, Science, 303, 1167

Beuther, H., Schilke, P., Menten, K. M., et al. 2002, ApJ, 566, 945

Bonnell, I. A., \& Bate, M. R. 2006, MNRAS, 370, 488

Bontemps, S., Motte, F., Csengeri, T., \& Schneider, N. 2010, A\&A, 524, A18

Breen, S. L., Ellingsen, S. P., Contreras, Y., et al. 2013, MNRAS, 435, 524

Brogan, C. L., Hunter, T. R., Cyganowski, C. J., et al. 2009, ApJ, 707, 1

Butler, M. J., \& Tan, J. C. 2012, ApJ, 754, 5

Carey, S. J., Noriega-Crespo, A., Mizuno, D. R., et al. 2009, PASP, 121, 76

Carral, P., Kurtz, S. E., Rodríguez, L. F., et al. 2002, AJ, 123, 2574

Caswell, J. L., Fuller, G. A., Green, J. A., et al. 2010, MNRAS, 404, 1029

Chapin, E. L., Berry, D. S., Gibb, A. G., et al. 2013, MNRAS, 430, 2545

Churchwell, E. 2002, ARA\&A, 40, 27

Combi, J. A., Albacete Colombo, J. F., Pellizza, L., et al. 2011, Ap\&SS, 331, 53

Csengeri, T., Bontemps, S., Schneider, N., Motte, F., \& Dib, S. 2011, A\&A, 527, A135

Csengeri, T., Urquhart, J. S., Schuller, F., et al. 2014, A\&A, 565, A75

Csengeri, T., Weiss, A., Wyrowski, F., et al. 2016, A\&A, 585, A104

Deharveng, L., Zavagno, A., Anderson, L. D., et al. 2012, A\&A, 546, A74

Didelon, P., Motte, F., Tremblin, P., et al. 2015, A\&A, 584, A4

Duarte-Cabral, A., Bontemps, S., Motte, F., et al. 2013, A\&A, 558, A125

Duarte-Cabral, A., Bontemps, S., Motte, F., et al. 2014, A\&A, 570, A1

Fallscheer, C., Reid, M. A., Di Francesco, J., et al. 2013, ApJ, 773, 102

Foster, J. B., Jackson, J. M., Barnes, P. J., et al. 2011, ApJS, 197, 25

Getman, K. V., Feigelson, E. D., Kuhn, M. A., et al. 2014, ApJ, 787, 108

Gezari, D. Y. 1982, ApJ, 259, L29

Giannini, T., Elia, D., Lorenzetti, D., et al. 2012, A\&A, 539, A156

Ginsburg, A., Bressert, E., Bally, J., \& Battersby, C. 2012, ApJ, 758, L29

Griffin, M. J., Abergel, A., Abreu, A., et al. 2010, A\&A, 518, L3

Gutermuth, R. A., \& Heyer, M. 2015, AJ, 149, 64

Hartmann, L., Ballesteros-Paredes, J., \& Heitsch, F. 2012, MNRAS, 420, 1457

Hennemann, M., Motte, F., Bontemps, S., et al. 2010, A\&A, 518, L84

Hennemann, M., Motte, F., Schneider, N., et al. 2012, A\&A, 543, L3

Hildebrand, R. H. 1983, QJRAS, 24, 267

Hill, T., Pinte, C., Minier, V., Burton, M. G., \& Cunningham, M. R. 2009, MNRAS, 392, 768

Hill, T., Motte, F., Didelon, P., et al. 2011, A\&A, 533, A94

Hill, T., Motte, F., Didelon, P., et al. 2012, A\&A, 542, A114

Holland, W. S., Bintley, D., Chapin, E. L., et al. 2013, MNRAS, 430, 2513

Kauffmann, J., Bertoldi, F., Bourke, T. L., Evans, II, N. J., \& Lee, C. W. 2008 A\&A, 487, 993

Kharchenko, N. V., Piskunov, A. E., Schilbach, E., Röser, S., \& Scholz, R.-D. 2013, A\&A, 558, A53

Könyves, V., André, P., Men'shchikov, A., et al. 2015, A\&A, 584, A91

Kraemer, K. E., \& Jackson, J. M. 1999, ApJS, 124, 439

Krumholz, M. R. 2015, in Very Massive Stars in the Local Universe, ed. J. S. Vink, Astrophys. Space Sci. Lib., 412, 43

Krumholz, M. R., \& McKee, C. F. 2008, Nature, 451, 1082

Krumholz, M. R., Klein, R. I., \& McKee, C. F. 2007, ApJ, 656, 959

Loughran, L., McBreen, B., Fazio, G. G., et al. 1986, ApJ, 303, 629

Louvet, F., Motte, F., Hennebelle, P., et al. 2014, A\&A, 570, A15

Louvet, F., Motte, F., Gusdorf, A., et al. 2016, A\&A, 595, A122

Markwardt, C. B. 2009, in Astronomical Data Analysis Software and Systems XVIII, eds. D. A. Bohlender, D. Durand, \& P. Dowler, ASP Conf. Ser., 411, 251

Marsh, K. A., Kirk, J. M., André, P., et al. 2016, MNRAS, 459, 342

Martin, P. G., Roy, A., Bontemps, S., et al. 2012, ApJ, 751, 28

Matthews, H. E., McCutcheon, W. H., Kirk, H., White, G. J., \& Cohen, M. 2008, AJ, 136, 2083

McKee, C. F., \& Tan, J. C. 2002, Nature, 416, 59

Men'shchikov, A. 2013, A\&A, 560, A63

Men'shchikov, A., André, P., Didelon, P., et al. 2012, A\&A, 542, A81

Morales, E. F. E., Wyrowski, F., Schuller, F., \& Menten, K. M. 2013, A\&A, 560, A76

Motte, F., \& André, P. 2001, A\&A, 365, 440

Motte, F., Andre, P., \& Neri, R. 1998, A\&A, 336, 150

Motte, F., Schilke, P., \& Lis, D. C. 2003, ApJ, 582, 277

Motte, F., Bontemps, S., Schilke, P., et al. 2007, A\&A, 476, 1243

Motte, F., Zavagno, A., Bontemps, S., et al. 2010, A\&A, 518, L77

Motte, F., Bontemps, S., \& Louvet, L. 2017, ARA\&A, 55, in press

Muñoz, D. J., Mardones, D., Garay, G., et al. 2007, ApJ, 668, 906

Nguyen Luong, Q., Motte, F., Hennemann, M., et al. 2011, A\&A, 535, A76
Nguyen-Luong, Q., Motte, F., Carlhoff, P., et al. 2013, ApJ, 775, 88 Ossenkopf, V., \& Henning, T. 1994, A\&A, 291, 943

Ott, S. 2010, in Astronomical Data Analysis Software and Systems XIX, ed.

Y. Mizumoto, K.-I. Morita, \& M. Ohishi, ASP Conf. Ser., 434, 139

Palmeirim, P., André, P., Kirk, J., et al. 2013, A\&A, 550, A38

Peretto, N., Fuller, G. A., Duarte-Cabral, A., et al. 2013, A\&A, 555, A112

Persi, P., \& Tapia, M. 2008, Star Formation in NGC 6334, ed. B. Reipurth, 456

Persi, P., \& Tapia, M. 2010, Mem. Soc. Astron. It., 81, 171

Pestalozzi, M. R., Minier, V., \& Booth, R. S. 2005, A\&A, 432, 737

Planck Collaboration I. 2011, A\&A, 536, A1

Poglitsch, A., Waelkens, C., Geis, N., et al. 2010, A\&A, 518, L2

Price, S. D., Egan, M. P., Carey, S. J., Mizuno, D. R., \& Kuchar, T. A. 2001, AJ, 121, 2819

Rodriguez, L. F., Canto, J., \& Moran, J. M. 1982, ApJ, 255, 103

Roussel, H. 2013, PASP, 125, 1126

Roy, A., André, P., Palmeirim, P., et al. 2014, A\&A, 562, A138

Russeil, D., Zavagno, A., Motte, F., et al. 2010, A\&A, 515, A55

Russeil, D., Zavagno, A., Adami, C., et al. 2013, A\&A, 538, A142

Russeil, D., Schneider, N., Anderson, L. D., et al. 2013, A\&A, 554, A42

Russeil, D., Tigé, J., Adami, C., et al. 2016, A\&A, 587, A135

Sandell, G. 2000, A\&A, 358, 242

Schneider, N., Csengeri, T., Bontemps, S., et al. 2010a, A\&A, 520, A49

Schneider, N., Motte, F., Bontemps, S., et al. 2010b, A\&A, 518, L83

Schneider, N., Csengeri, T., Hennemann, M., et al. 2012, A\&A, 540, L11

Schneider, N., Bontemps, S., Girichidis, P., et al. 2015, MNRAS, 453, 41

Schuller, F., Menten, K. M., Contreras, Y., et al. 2009, A\&A, 504, 415

Svoboda, B. E., Shirley, Y. L., Battersby, C., et al. 2016, ApJ, 822, 59

Tackenberg, J., Beuther, H., Henning, T., et al. 2012, A\&A, 540, A113

Tan, J. C., Kong, S., Butler, M. J., Caselli, P., \& Fontani, F. 2013, ApJ, 779, 96

Tan, J. C., Beltrán, M. T., Caselli, P., et al. 2014, Protostars and Planets VI, 149

Tan, J. C., Kong, S., Zhang, Y., et al. 2016, ApJ, 821, L3

Tapia, M., Roth, M., Lopez, J. A., et al. 1991, A\&A, 242, 388

Tapia, M., Rodríguez, L. F., Persi, P., Roth, M., \& Gómez, M. 2009, AJ, 137, 4127

Wang, K., Zhang, Q., Testi, L., et al. 2014, MNRAS, 439, 3275

Ward-Thompson, D., Motte, F., \& Andre, P. 1999, MNRAS, 305, 143

White, R. L., Becker, R. H., \& Helfand, D. J. 2005, AJ, 130, 586

Willis, S., Marengo, M., Allen, L., et al. 2013, ApJ, 778, 96

Wright, E. L., Eisenhardt, P. R. M., Mainzer, A. K., et al. 2010, AJ, 140, 1868

Zavagno, A., Pomarès, M., Deharveng, L., et al. 2007, A\&A, 472, 835

Zernickel, A., Schilke, P., \& Smith, R. J. 2013, A\&A, 554, L2

1 Aix-Marseille Univ., CNRS, LAM, Laboratoire d'Astrophysique de Marseille, Marseille, France

e-mail: jeremy.tige@lam. fr

2 Université Grenoble Alpes, CNRS-INSU, Institut de Planétologie et d'Astrophysique de Grenoble, 38000 Grenoble, France

3 Laboratoire AIM Paris-Saclay, CEA/IRFU - CNRS/INSU Université Paris Diderot, Service d'Astrophysique, Bât. 709,

CEA-Saclay, 91191 Gif-sur-Yvette Cedex, France

4 Max-Planck-Institut für Astronomie, Königsstuhl 17,

69117 Heidelberg, Germany

5 Laboratoire d'Astrophysique de Bordeaux, Univ. Bordeaux, CNRS, B18N, Allée G. Saint-Hilaire, 33615 Pessac, France

${ }^{6}$ I. Physik. Institut, University of Cologne, 50937 Cologne, Germany

7 Joint ALMA Observatory, 3107 Alonso de Cordova, Vitacura, Santiago, Chile

${ }^{8}$ Korea Astronomy and Space Science Institute, 776 Daedeok daero, Yuseoung, Daejeon 34055, Korea

9 NAOJ Chile Observatory, National Astronomical Observatory of Japan, 2-21-1 Osawa, Mitaka, Tokyo 181-8588, Japan

10 National Research Council Canada, Herzberg Institute of Astrophysics, 5071 West Saanich Road, Victoria, BC V9E 2E7, Canada

11 Department of Physics and Astronomy, University of Victoria, PO Box 355, STN CSC, Victoria, BC V8W 3P6, Canada

12 Department of Astronomy, Universidad de Chile, Las Condes, Santiago, Chile

13 Department of Physics and Astronomy, West Virginia University, Morgantown, WV 26506, USA

14 National Radio Astronomy Observatory, PO Box 2, Green Bank, WV 24944, USA

15 INAF-Istituto di Astrofisica e Planetologia Spaziali, via Fosso del Cavaliere 100, 00133 Rome, Italy 
16 Université de Toulouse, UPS-OMP, CNRS, IRAP, 31028 Toulouse Cedex 4, France

17 Jeremiah Horrocks Institute, University of Central Lancashire, Preston, Lancashire, PR1 2HE, UK

18 Canadian Institute for Theoretical Astrophysics, University of Toronto, 60 St. George Street, Toronto, ON M5S 3H8, Canada

19 Departamento de Fisica, Universidad de Atacama, Copiapo, Chile

${ }^{20}$ Cardiff School of Physics and Astronomy, Cardiff University, Queen's Buildings, The Parade, Cardiff, Wales, CF24 3AA, UK
${ }^{21}$ European Space Astronomy Centre ESA/ESAC, 28691 Villanueva de la Cañada, Madrid, Spain

22 Institut d'Astrophysique de Paris, Sorbonne Universités, UPMC Univ. Paris 06, CNRS UMR 7095, 75014 Paris, France

23 INAF-IRA, via P. Gobetti 101, 40129 Bologna, Italy

24 Department of Physics and Astronomy, The Open University, Walton Hall, Milton Keynes, MK7 6AA, UK

25 RAL Space, STFC Rutherford Appleton Laboratory, Chilton, Didcot, Oxfordshire, OX11 0QX, UK 
Appendix A: HOBYS catalogs for the 46 MDCs found in NGC 6334

We present in this appendix the flux catalogs for the 46 MDCs discussed in the text.

Catalog entries are as follows:

1. Getsources source number.

2. Name $=$ HOBYS_J prefix directly followed by a tag created from the J2000 sexagesimal coordinates.

3. Flag for the fluxes reliability and upper limit (see criteria in Sect. 3.2).

" 0 " means that the flux is considered unreliable because the source is too weak, $S^{\text {peak }} / \sigma<2$ and/or $S^{\text {int }} / \sigma<2$. Its error, $2 \sigma$, is used as an upper limit on SEDs when $\lambda \geq 160 \mu \mathrm{m}$ and unused otherwise (see Sect. 4.2).
" 1 " means that the flux is considered reliable. It is used for SED fits when $\lambda \geq 160 \mu \mathrm{m}$ and when $\lambda \geq 70 \mu \mathrm{m}$ for $T_{\text {dust }}>$ $32 \mathrm{~K}$.

" 2 " means that the flux is not considered reliable because the source is too large, $F W H M>0.3 \mathrm{pc}$, and/or too elliptical, $e>2$. Its estimated flux is used as an upper limit on SEDs when $\lambda \geq 160 \mu \mathrm{m}$ or when $\lambda \geq 70 \mu \mathrm{m}$ for $T_{\text {dust }}>32 \mathrm{~K}$ and it is unused otherwise.

4. Estimate of the peak flux and its error in Jy/beam.

5. Estimate of the integrated flux and its error in Jy. Flux scaling has not been applied.

6. Major/minor (non-deconvolved) size estimate (FWHM) of the source in arcseconds.

7. Position angle of the major axis, east of north, in degrees. 
Table A.1. HOBYS catalog of Herschel fluxes extracted by getsources for the 46 MDCs found in NGC 6334

\begin{tabular}{|c|c|c|c|}
\hline $\begin{array}{l}\text { Number } \\
\text { getsources } \\
\text { (1) }\end{array}$ & $\begin{array}{c}\text { Name } \\
\text { HOBYS_J } \\
(2)\end{array}$ & $\begin{array}{l}\mathrm{RA}_{2000} \\
(\mathrm{~h} \mathrm{~m} \mathrm{~s})\end{array}$ & $\begin{array}{c}\operatorname{Dec}_{2000} \\
\left({ }^{\circ} \prime^{\prime \prime}\right)\end{array}$ \\
\hline 1 & $172053.4-354702$ & $17: 20: 53.47$ & $-35: 47: 02.3$ \\
\hline 2 & $172055.2-354500$ & $17: 20: 55.23$ & $-35: 45: 00.0$ \\
\hline 3 & $172056.4-354528$ & $17: 20: 56.48$ & $-35: 45: 28.9$ \\
\hline 4 & $172053.6-354521$ & $17: 20: 53.66$ & $-35: 45: 21.1$ \\
\hline 5 & $172053.0-354317$ & $17: 20: 53.03$ & $-35: 43: 17.0$ \\
\hline 6 & $171957.5-355752$ & $17: 19: 57.55$ & $-35: 57: 52.1$ \\
\hline 7 & $171955.7-355749$ & $17: 19: 55.74$ & $-35: 57: 49.9$ \\
\hline 8 & $172057.4-354517$ & $17: 20: 57.47$ & $-35: 45: 17.8$ \\
\hline 9 & $172023.0-355443$ & $17: 20: 23.05$ & $-35: 54: 43.6$ \\
\hline 10 & $172018.0-355456$ & $17: 20: 18.04$ & $-35: 54: 56.5$ \\
\hline 11 & $172002.1-361214$ & $17: 20: 02.16$ & $-36: 12: 14.5$ \\
\hline 12 & $172020.8-355432$ & $17: 20: 20.86$ & $-35: 54: 32.9$ \\
\hline 13 & $171907.2-360820$ & $17: 19: 07.22$ & $-36: 08: 20.2$ \\
\hline 14 & $172054.6-354511$ & $17: 20: 54.67$ & $-35: 45: 11.0$ \\
\hline 15 & $171954.7-355739$ & $17: 19: 54.70$ & $-35: 57: 39.1$ \\
\hline 16 & $171905.2-360757$ & $17: 19: 05.24$ & $-36: 07: 57.8$ \\
\hline 17 & $171951.8-355707$ & $17: 19: 51.86$ & $-35: 57: 07.7$ \\
\hline 18 & $172228.8-350933$ & $17: 22: 28.89$ & $-35: 09: 33.8$ \\
\hline 19 & $172022.9-355500$ & $17: 20: 22.97$ & $-35: 55: 00.3$ \\
\hline 20 & $172023.8-355450$ & $17: 20: 23.83$ & $-35: 54: 50.4$ \\
\hline 21 & $172030.9-360054$ & $17: 20: 30.91$ & $-36: 00: 54.8$ \\
\hline 22 & $171700.4-362050$ & $17: 17: 00.41$ & $-36: 20: 50.2$ \\
\hline 23 & $171701.8-362045$ & $17: 17: 01.82$ & $-36: 20: 45.7$ \\
\hline 24 & $171834.8-360846$ & $17: 18: 34.83$ & $-36: 08: 46.0$ \\
\hline 25 & $172019.2-355422$ & $17: 20: 19.28$ & $-35: 54: 22.8$ \\
\hline 26 & $172055.3-354605$ & $17: 20: 55.36$ & $-35: 46: 05.7$ \\
\hline 27 & $171939.6-355652$ & $17: 19: 39.66$ & $-35: 56: 52.0$ \\
\hline 28 & $172049.1-354500$ & $17: 20: 49.10$ & $-35: 45: 00.6$ \\
\hline 29 & 171954.9-355755 & $17: 19: 54.98$ & $-35: 57: 55.5$ \\
\hline 30 & $171709.0-361903$ & $17: 17: 09.03$ & $-36: 19: 03.3$ \\
\hline 31 & $171745.8-360854$ & $17: 17: 45.81$ & $-36: 08: 54.8$ \\
\hline 32 & $172019.5-355440$ & $17: 20: 19.50$ & $-35: 54: 40.4$ \\
\hline 33 & $172233.4-351316$ & $17: 22: 33.45$ & $-35: 13: 16.7$ \\
\hline 34 & $172054.5-353518$ & $17: 20: 54.57$ & $-35: 35: 18.3$ \\
\hline 35 & 171958.0-361313 & $17: 19: 58.00$ & $-36: 13: 13.5$ \\
\hline 36 & $172017.7-355906$ & $17: 20: 17.70$ & $-35: 59: 06.5$ \\
\hline 37 & $171748.0-361102$ & $17: 17: 48.01$ & $-36: 11: 02.5$ \\
\hline 38 & $172018.8-355435$ & $17: 20: 18.88$ & $-35: 54: 35.3$ \\
\hline 39 & $171733.9-362613$ & $17: 17: 33.93$ & $-36: 26: 13.1$ \\
\hline 40 & $172019.7-355633$ & $17: 20: 19.72$ & $-35: 56: 33.9$ \\
\hline 41 & $172017.9-355446$ & $17: 20: 17.96$ & $-35: 54: 46.8$ \\
\hline 42 & $172204.1-352454$ & $17: 22: 04.14$ & $-35: 24: 54.6$ \\
\hline 43 & $172059.4-354457$ & $17: 20: 59.48$ & $-35: 44: 57.2$ \\
\hline 44 & $172225.5-351659$ & $17: 22: 25.58$ & $-35: 16: 59.9$ \\
\hline 45 & $171747.9-360928$ & $17: 17: 47.95$ & $-36: 09: 28.5$ \\
\hline 46 & $172024.0-355459$ & $17: 20: 24.00$ & $-35: 54: 59.7$ \\
\hline
\end{tabular}


Table A.2. Same as Table A.1 for Herschel-70 $\mu \mathrm{m}$.

\begin{tabular}{|c|c|c|c|c|c|c|}
\hline $\begin{array}{c}\text { Number } \\
\text { Id }\end{array}$ & $\begin{array}{c}\operatorname{Tag}_{070} \\
\text { (3) }\end{array}$ & $\begin{array}{c}S_{070}^{\text {peak }} \\
(\mathrm{Jy} / \text { beam }) \\
(4)\end{array}$ & $\begin{array}{l}S_{070}^{\mathrm{int}} \\
(\mathrm{Jy}) \\
(5)\end{array}$ & $\begin{array}{c}F W H M_{070}^{\text {maj }} \\
(\operatorname{arcsec}) \\
(6)\end{array}$ & $\begin{array}{c}F W H M_{070}^{\min } \\
(\operatorname{arcsec}) \\
(6)\end{array}$ & $\begin{array}{c}\mathrm{PA}_{070} \\
\text { (degrees) } \\
(7)\end{array}$ \\
\hline 1 & 1 & $1560 \pm 20$ & $11700 \pm 60$ & 14.7 & 12.1 & 178 \\
\hline 2 & 2 & $2.9 \pm 1.0$ & $49.8 \pm 2.6$ & 21.2 & 15.4 & 174 \\
\hline 3 & 2 & $0.9 \pm 0.2$ & $2.9 \pm 0.3$ & 14.3 & 6.9 & 102 \\
\hline 4 & 0 & & & & & \\
\hline 5 & 0 & & & & & \\
\hline 6 & 2 & $1160 \pm 3$ & $14100 \pm 20$ & 19.8 & 13.4 & 124 \\
\hline 7 & 0 & & & & & \\
\hline 8 & 0 & & & & & \\
\hline 9 & 0 & & & & & \\
\hline 10 & 1 & $268 \pm 59$ & $765 \pm 96$ & 10.8 & 7.7 & 110 \\
\hline 11 & 0 & & & & & \\
\hline 12 & 0 & & & & & \\
\hline 13 & 0 & & & & & \\
\hline 14 & 1 & $98.1 \pm 1.8$ & $311 \pm 4$ & 11.9 & 6.1 & 98 \\
\hline 15 & 2 & $9.7 \pm 3.2$ & $213 \pm 8$ & 24.4 & 16.6 & 61 \\
\hline 16 & 0 & & & & & \\
\hline 17 & 0 & & & & & \\
\hline 18 & 0 & & & & & \\
\hline 19 & 0 & & & & & \\
\hline 20 & 0 & & & & & \\
\hline 21 & 0 & & & & & \\
\hline 22 & 0 & & & & & \\
\hline 23 & 0 & & & & & \\
\hline 24 & 0 & & & & & \\
\hline 25 & 0 & & & & & \\
\hline 26 & 0 & & & & & \\
\hline 27 & 0 & & & & & \\
\hline 28 & 0 & & & & & \\
\hline 29 & 1 & $57.8 \pm 18.6$ & $113 \pm 23$ & 8.0 & 7.5 & 125 \\
\hline 30 & 0 & & & & & \\
\hline 31 & 0 & & & & & \\
\hline 32 & 1 & $99.3 \pm 41.3$ & $238 \pm 46$ & 10.3 & 6.5 & 139 \\
\hline 33 & 0 & & & & & \\
\hline 34 & 0 & & & & & \\
\hline 35 & 0 & & & & & \\
\hline 36 & 1 & $0.8 \pm 0.2$ & $0.7 \pm 0.3$ & 6.3 & 5.9 & 54 \\
\hline 37 & 0 & & & & & \\
\hline 38 & 2 & $336 \pm 2$ & $6380 \pm 14$ & 22.9 & 21.3 & 50 \\
\hline 39 & 0 & & & & & \\
\hline 40 & 0 & & & & & \\
\hline 41 & 1 & $153 \pm 45$ & $266 \pm 49$ & 11.1 & 5.9 & 86 \\
\hline 42 & 0 & & & & & \\
\hline 43 & 1 & $0.8 \pm 0.3$ & $2.7 \pm 0.3$ & 13.2 & 10.4 & 146 \\
\hline 44 & 0 & & & & & \\
\hline 45 & 0 & & & & & \\
\hline 46 & 1 & $186 \pm 12$ & $304 \pm 15$ & 6.3 & 5.9 & 89 \\
\hline
\end{tabular}


Table A.3. Same as Table A.1 for Herschel- $160 \mu \mathrm{m}$.

\begin{tabular}{|c|c|c|c|c|c|c|}
\hline $\begin{array}{c}\text { Number } \\
\text { Id }\end{array}$ & $\operatorname{Tag}_{160}$ & $\begin{array}{c}S_{160}^{\text {peak }} \\
\text { (Jy/beam) }\end{array}$ & $\begin{array}{l}S_{160}^{\text {int }} \\
(\mathrm{Jy})\end{array}$ & $\begin{array}{c}F W H M_{160}^{\mathrm{maj}} \\
(\operatorname{arcsec})\end{array}$ & $\begin{array}{c}F W H M_{160}^{\min } \\
(\operatorname{arcsec})\end{array}$ & $\begin{array}{c}\mathrm{PA}_{160} \\
\text { (degrees) }\end{array}$ \\
\hline 1 & 1 & $2180 \pm 56$ & $9660 \pm 116$ & 23.6 & 20.0 & 94 \\
\hline 2 & 1 & $167 \pm 29$ & $468 \pm 35$ & 22.8 & 13.2 & 169 \\
\hline 3 & 1 & $111 \pm 44$ & $271 \pm 44$ & 19.1 & 11.7 & 50 \\
\hline 4 & 2 & $26.4 \pm 46.9$ & $14.8 \pm 46.9$ & 11.7 & 11.7 & 108 \\
\hline 5 & 1 & $30.4 \pm 6.7$ & $97.2 \pm 10.9$ & 21.1 & 17.0 & 19 \\
\hline 6 & 1 & $2240 \pm 39$ & $7450 \pm 68$ & 19.3 & 17.6 & 145 \\
\hline 7 & 1 & $720 \pm 41$ & $1310 \pm 41$ & 16.0 & 13.6 & 35 \\
\hline 8 & 2 & $39.5 \pm 25.4$ & $48.0 \pm 25.4$ & 14.9 & 11.7 & 179 \\
\hline 9 & 1 & $148 \pm 18$ & $277 \pm 23$ & 19.5 & 11.7 & 134 \\
\hline 10 & 1 & $937 \pm 19$ & $2080 \pm 19$ & 18.4 & 16.6 & 112 \\
\hline 11 & 2 & $3.8 \pm 2.0$ & $32.6 \pm 5.2$ & 56.5 & 21.1 & 53 \\
\hline 12 & 1 & $497 \pm 20$ & $832 \pm 20$ & 20.2 & 11.7 & 2 \\
\hline 13 & 2 & $0.3 \pm 0.3$ & $1.7 \pm 0.6$ & 32.8 & 29.0 & 88 \\
\hline 14 & 1 & $858 \pm 35$ & $1262 \pm 35$ & 14.5 & 11.7 & 119 \\
\hline 15 & 1 & $225 \pm 43$ & $553 \pm 52$ & 21.6 & 13.7 & 101 \\
\hline 16 & 2 & $-1.0 \pm 0.4$ & $-4.8 \pm 0.6$ & 33.1 & 21.4 & 134 \\
\hline 17 & 1 & $45.6 \pm 14.1$ & $111 \pm 19$ & 24.9 & 15.5 & 172 \\
\hline 18 & 2 & $0.7 \pm 0.3$ & $17.0 \pm 0.9$ & 65.5 & 42.8 & 61 \\
\hline 19 & 2 & $47.8 \pm 4.9$ & $38.4 \pm 4.9$ & 11.7 & 11.7 & 137 \\
\hline 20 & 1 & $102 \pm 17$ & $229 \pm 17$ & 20.2 & 15.7 & 129 \\
\hline 21 & 1 & $15.3 \pm 1.3$ & $163 \pm 4$ & 42.1 & 31.6 & 8 \\
\hline 22 & 2 & $4.0 \pm 9.0$ & $-2.7 \pm 9.0$ & 11.7 & 11.7 & 64 \\
\hline 23 & 1 & $163 \pm 36$ & $231 \pm 36$ & 15.2 & 11.7 & 68 \\
\hline 24 & 1 & $14.2 \pm 0.9$ & $108 \pm 2$ & 31.7 & 24.8 & 109 \\
\hline 25 & 1 & $123 \pm 20$ & $204 \pm 20$ & 19.3 & 11.7 & 91 \\
\hline 26 & 2 & $29.5 \pm 64.0$ & $43.7 \pm 64.2$ & 17.5 & 11.7 & 168 \\
\hline 28 & 2 & $36.2 \pm 24.9$ & $64.7 \pm 32.9$ & 25.2 & 11.7 & 23 \\
\hline 27 & 1 & $16.6 \pm 2.8$ & $30.5 \pm 2.8$ & 15.6 & 12.7 & 173 \\
\hline 29 & 1 & $458 \pm 38$ & $918 \pm 38$ & 17.0 & 14.7 & 110 \\
\hline 30 & 1 & $8.9 \pm 2.4$ & $34.4 \pm 4.3$ & 27.1 & 16.8 & 86 \\
\hline 31 & 1 & $2.4 \pm 0.8$ & $8.2 \pm 1.0$ & 28.7 & 15.3 & 133 \\
\hline 32 & 1 & $818 \pm 22$ & $1290 \pm 22$ & 19.2 & 11.7 & 82 \\
\hline 33 & 2 & $2.5 \pm 0.7$ & $6.9 \pm 0.9$ & 28.5 & 11.7 & 158 \\
\hline 34 & 2 & $2.9 \pm 1.9$ & $5.4 \pm 2.4$ & 24.4 & 13.7 & 174 \\
\hline 35 & 2 & $7.9 \pm 1.6$ & $26.8 \pm 3.4$ & 47.4 & 14.9 & 175 \\
\hline 36 & 1 & $37.5 \pm 11.7$ & $61.0 \pm 11.7$ & 19.4 & 11.7 & 76 \\
\hline 37 & 2 & $2.1 \pm 0.8$ & $6.2 \pm 1.2$ & 40.8 & 11.9 & 86 \\
\hline 38 & 1 & $655 \pm 20$ & $2870 \pm 56$ & 25.1 & 18.0 & 77 \\
\hline 39 & 1 & $1.9 \pm 0.8$ & $7.0 \pm 1.5$ & 19.5 & 18.1 & 172 \\
\hline 40 & 1 & $37.9 \pm 5.7$ & $98.7 \pm 8.2$ & 25.1 & 13.1 & 69 \\
\hline 41 & 1 & $530 \pm 20$ & $588 \pm 20$ & 11.7 & 11.7 & 165 \\
\hline 42 & 1 & $2.4 \pm 0.4$ & $15.5 \pm 1.0$ & 34.0 & 19.6 & 78 \\
\hline 43 & 1 & $13.8 \pm 5.2$ & $24.0 \pm 5.2$ & 16.6 & 13.9 & 161 \\
\hline 44 & 2 & $0.6 \pm 0.2$ & $11.2 \pm 0.9$ & 56.3 & 53.4 & 110 \\
\hline 45 & 2 & $5.0 \pm 1.2$ & $18.9 \pm 1.7$ & 31.8 & 14.7 & 100 \\
\hline 46 & 1 & $535 \pm 17$ & $710 \pm 17$ & 12.5 & 11.7 & 75 \\
\hline
\end{tabular}


Table A.4. Same as Table A.1 for Herschel-250 $\mu \mathrm{m}$

\begin{tabular}{|c|c|c|c|c|c|c|}
\hline $\begin{array}{l}\text { Number } \\
\text { Id }\end{array}$ & $\operatorname{Tag}_{250}$ & $\begin{array}{c}S_{250}^{\text {peak }} \\
\text { (Jy/beam) }\end{array}$ & $\begin{array}{l}S_{250}^{\text {int }} \\
(\mathrm{Jy})\end{array}$ & $\begin{array}{c}F W H M_{250}^{\mathrm{maj}} \\
(\operatorname{arcsec})\end{array}$ & $\begin{array}{c}F W H M_{250}^{\min } \\
(\operatorname{arcsec})\end{array}$ & $\begin{array}{c}\mathrm{PA}_{250} \\
\text { (degrees) }\end{array}$ \\
\hline 1 & 1 & $2660 \pm 38$ & $3320 \pm 39$ & 20.5 & 18.2 & 50 \\
\hline 2 & 1 & $666 \pm 37$ & $781 \pm 32$ & 19.9 & 18.2 & 159 \\
\hline 3 & 1 & $445 \pm 42$ & $452 \pm 37$ & 19.3 & 18.2 & 62 \\
\hline 4 & 1 & $139 \pm 41$ & $135 \pm 36$ & 18.2 & 18.2 & 12 \\
\hline 5 & 1 & $118 \pm 13$ & $128 \pm 13$ & 18.2 & 18.2 & 32 \\
\hline 6 & 1 & $1530 \pm 4$ & $1910 \pm 3$ & 22.0 & 18.2 & 51 \\
\hline 7 & 1 & $605 \pm 4$ & $689 \pm 3$ & 19.1 & 18.2 & 22 \\
\hline 8 & 1 & $114 \pm 43$ & $112 \pm 38$ & 18.7 & 18.2 & 137 \\
\hline 9 & 1 & $263 \pm 6$ & $269 \pm 6$ & 18.2 & 18.2 & 156 \\
\hline 10 & 1 & $441 \pm 5$ & $515 \pm 4$ & 19.0 & 18.2 & 59 \\
\hline 11 & 1 & $13.6 \pm 2.2$ & $61.3 \pm 4.0$ & 49.8 & 29.3 & 53 \\
\hline 12 & 1 & $411 \pm 6$ & $456 \pm 5$ & 19.3 & 18.2 & 162 \\
\hline 13 & 1 & $4.1 \pm 1.9$ & $8.6 \pm 2.0$ & 31.6 & 24.2 & 63 \\
\hline 14 & 1 & $675 \pm 40$ & $667 \pm 36$ & 18.2 & 18.2 & 117 \\
\hline 15 & 1 & $339 \pm 4$ & $477 \pm 3$ & 33.1 & 18.2 & 100 \\
\hline 16 & 1 & $5.2 \pm 1.7$ & $8.4 \pm 1.7$ & 33.2 & 18.9 & 90 \\
\hline 17 & 1 & $93.0 \pm 4.1$ & $123 \pm 4$ & 24.8 & 19.0 & 175 \\
\hline 18 & 1 & $5.6 \pm 1.4$ & $21.0 \pm 2.3$ & 48.1 & 26.8 & 75 \\
\hline 19 & 1 & $133 \pm 6$ & $139 \pm 5$ & 18.2 & 18.2 & 20 \\
\hline 20 & 1 & $224 \pm 6$ & $245 \pm 5$ & 20.2 & 18.2 & 100 \\
\hline 21 & 1 & $31.7 \pm 2.3$ & $117.8 \pm 4$ & 43.2 & 30.5 & 4 \\
\hline 22 & 1 & $88.7 \pm 11.3$ & $89.5 \pm 10.0$ & 19.5 & 18.2 & 31 \\
\hline 23 & 1 & $264 \pm 13$ & $274 \pm 11$ & 18.2 & 18.2 & 75 \\
\hline 24 & 1 & $28.9 \pm 1.4$ & $80.5 \pm 2.0$ & 32.2 & 25.0 & 93 \\
\hline 25 & 1 & $337 \pm 6$ & $351 \pm 5$ & 19.8 & 18.2 & 111 \\
\hline 26 & 1 & $187 \pm 38$ & $194 \pm 34$ & 20.1 & 18.2 & 105 \\
\hline 27 & 1 & $31.9 \pm 2.7$ & $47.4 \pm 2.4$ & 31.6 & 18.9 & 139 \\
\hline 28 & 1 & $133 \pm 37$ & $149 \pm 35$ & 22.5 & 18.2 & 30 \\
\hline 29 & 1 & $120 \pm 4$ & $252 \pm 3$ & 28.8 & 24.6 & 160 \\
\hline 30 & 1 & $23.6 \pm 3.0$ & $42.1 \pm 3.4$ & 27.3 & 20.4 & 76 \\
\hline 31 & 2 & $5.2 \pm 2.6$ & $10.1 \pm 2.5$ & 34.2 & 21.8 & 139 \\
\hline 32 & 1 & $402 \pm 6$ & $400 \pm 5$ & 18.2 & 18.2 & 68 \\
\hline 33 & 1 & $12.8 \pm 1.3$ & $19.0 \pm 1.6$ & 25.8 & 19.9 & 162 \\
\hline 34 & 1 & $12.8 \pm 5.5$ & $15.8 \pm 4.9$ & 24.5 & 20.3 & 92 \\
\hline 35 & 1 & $21.3 \pm 1.4$ & $59.1 \pm 2.1$ & 42.8 & 21.7 & 164 \\
\hline 36 & 1 & $80.0 \pm 5.9$ & $105 \pm 5$ & 25.4 & 18.2 & 90 \\
\hline 37 & 1 & $9.8 \pm 2.7$ & $16.2 \pm 2.9$ & 31.7 & 18.2 & 78 \\
\hline 38 & 1 & $348 \pm 5$ & $365 \pm 9$ & 18.2 & 18.2 & 104 \\
\hline 39 & 1 & $6.7 \pm 1.6$ & $12.3 \pm 1.6$ & 34.1 & 23.9 & 167 \\
\hline 40 & 1 & $61.1 \pm 5.0$ & $84.1 \pm 5.0$ & 29.0 & 18.2 & 79 \\
\hline 41 & 1 & $416 \pm 4$ & $403 \pm 4$ & 18.2 & 18.2 & 68 \\
\hline 42 & 1 & $9.0 \pm 0.9$ & $37.3 \pm 1.7$ & 37.7 & 33.4 & 52 \\
\hline 43 & 1 & $71.9 \pm 21$ & $78 \pm 19$ & 19.4 & 18.2 & 54 \\
\hline 44 & 1 & $4.1 \pm 1.0$ & $15.9 \pm 1.8$ & 49.1 & 31.8 & 137 \\
\hline 45 & 1 & $22.2 \pm 2.6$ & $35.6 \pm 2.7$ & 27.0 & 18.2 & 176 \\
\hline 46 & 1 & $331 \pm 6$ & $376 \pm 5$ & 20.1 & 18.2 & 103 \\
\hline
\end{tabular}


Table A.5. Same as Table A.1 for Herschel-350 $\mu \mathrm{m}$.

\begin{tabular}{|c|c|c|c|c|c|c|}
\hline $\begin{array}{c}\text { Number } \\
\text { Id }\end{array}$ & $\operatorname{Tag}_{350}$ & $\begin{array}{c}S_{350}^{\text {peak }} \\
(\text { Jy/beam) }\end{array}$ & $\begin{array}{l}S_{350}^{\text {int }} \\
(\mathrm{Jy})\end{array}$ & $\begin{array}{c}A F W H M_{350} \\
\quad(\operatorname{arcsec})\end{array}$ & $\begin{array}{c}F W H M_{350}^{\min } \\
(\operatorname{arcsec})\end{array}$ & $\begin{array}{c}\mathrm{PA}_{350} \\
\text { (degrees) }\end{array}$ \\
\hline 1 & 1 & $1600 \pm 30$ & $1610 \pm 28$ & 24.9 & 24.9 & 33 \\
\hline 2 & 1 & $544 \pm 22$ & $603 \pm 20$ & 24.9 & 24.9 & 113 \\
\hline 3 & 1 & $389 \pm 28$ & $393 \pm 26$ & 24.9 & 24.9 & 34 \\
\hline 4 & 1 & $163 \pm 29$ & $166 \pm 27$ & 24.9 & 24.9 & 28 \\
\hline 5 & 1 & $111 \pm 11$ & $107 \pm 10$ & 24.9 & 24.9 & 177 \\
\hline 6 & 1 & $743 \pm 7$ & $735 \pm 6$ & 24.9 & 24.9 & 15 \\
\hline 7 & 1 & $359 \pm 7$ & $358 \pm 6$ & 24.9 & 24.9 & 69 \\
\hline 8 & 1 & $94.0 \pm 21.8$ & $124 \pm 20$ & 30.4 & 24.9 & 156 \\
\hline 9 & 1 & $176 \pm 5$ & $165 \pm 4$ & 24.9 & 24.9 & 160 \\
\hline 10 & 1 & $266 \pm 4$ & $292 \pm 4$ & 26.5 & 24.9 & 27 \\
\hline 11 & 1 & $14.4 \pm 2.2$ & $42.4 \pm 3.2$ & 50.8 & 32.6 & 52 \\
\hline 12 & 1 & $218 \pm 5$ & $217 \pm 4$ & 24.9 & 24.9 & 71 \\
\hline 13 & 1 & $5.9 \pm 2.4$ & $7.6 \pm 2.2$ & 34.1 & 24.9 & 71 \\
\hline 14 & 1 & $435 \pm 29$ & $447 \pm 26$ & 24.9 & 24.9 & 164 \\
\hline 15 & 1 & $276 \pm 7$ & $337 \pm 6.0$ & 32.0 & 24.9 & 102 \\
\hline 16 & 1 & $9.2 \pm 2.3$ & $11.4 \pm 2.1$ & 27.7 & 25.6 & 96 \\
\hline 17 & 1 & $71.9 \pm 7.1$ & $85.9 \pm 6.5$ & 36.0 & 24.9 & 107 \\
\hline 18 & 1 & $7.4 \pm 1.0$ & $14.5 \pm 1.2$ & 51.3 & 26.1 & 58 \\
\hline 19 & 1 & $87.9 \pm 4.7$ & $82.6 \pm 4.2$ & 24.9 & 24.9 & 150 \\
\hline 20 & 1 & $117 \pm 4$ & $114 \pm 4$ & 24.9 & 24.9 & 132 \\
\hline 21 & 1 & $30.9 \pm 1.1$ & $73.6 \pm 1.4$ & 39.2 & 33.3 & 166 \\
\hline 22 & 1 & $69.0 \pm 2.7$ & $66.5 \pm 2.4$ & 24.9 & 24.9 & 173 \\
\hline 23 & 1 & $151 \pm 3$ & $146 \pm 3$ & 24.9 & 24.9 & 63 \\
\hline 24 & 1 & $24.9 \pm 1.2$ & $70.6 \pm 2.2$ & 44.7 & 30.3 & 70 \\
\hline 25 & 1 & $194 \pm 4$ & $158 \pm 4$ & 24.9 & 24.9 & 97 \\
\hline 26 & 1 & $88.6 \pm 29.9$ & $137 \pm 27$ & 37.2 & 24.9 & 101 \\
\hline 27 & 1 & $28.8 \pm 2.3$ & $42.7 \pm 2.1$ & 43.1 & 24.9 & 150 \\
\hline 28 & 1 & $102 \pm 28$ & $109 \pm 26$ & 24.9 & 24.9 & 67 \\
\hline 29 & 1 & $92.5 \pm 6.5$ & $91.2 \pm 5.9$ & 24.9 & 24.9 & 143 \\
\hline 30 & 1 & $23.8 \pm 1.7$ & $31.9 \pm 1.6$ & 41.2 & 24.9 & 99 \\
\hline 31 & 1 & $7.0 \pm 2.3$ & $8.9 \pm 2.1$ & 30.6 & 25.1 & 114 \\
\hline 32 & 1 & $123 \pm 5$ & $117 \pm 4$ & 24.9 & 24.9 & 47 \\
\hline 33 & 1 & $16.5 \pm 1.0$ & $20.2 \pm 1.0$ & 28.9 & 24.9 & 173 \\
\hline 34 & 1 & $14.9 \pm 2.8$ & $14.9 \pm 2.5$ & 27.7 & 24.9 & 50 \\
\hline 35 & 1 & $17.5 \pm 1.3$ & $44.1 \pm 1.9$ & 40.8 & 33.8 & 157 \\
\hline 36 & 1 & $76.1 \pm 3.5$ & $98.9 \pm 3.2$ & 29.4 & 24.9 & 131 \\
\hline 37 & 1 & $8.8 \pm 1.9$ & $10.5 \pm 1.8$ & 31.6 & 24.9 & 97 \\
\hline 38 & 1 & $203 \pm 5$ & $197 \pm 6$ & 24.9 & 24.9 & 135 \\
\hline 39 & 1 & $6.8 \pm 0.9$ & $9.6 \pm 0.8$ & 30.7 & 27.5 & 165 \\
\hline 40 & 1 & $39.4 \pm 4.6$ & $43.6 \pm 4.2$ & 29.7 & 24.9 & 75 \\
\hline 41 & 1 & $157 \pm 4$ & $148 \pm 4$ & 24.9 & 24.9 & 63 \\
\hline 42 & 1 & $7.6 \pm 0.8$ & $15.7 \pm 0.9$ & 47.9 & 27.9 & 53 \\
\hline 43 & 1 & $77.0 \pm 15.5$ & $83.7 \pm 14.2$ & 26.1 & 24.9 & 27.5 \\
\hline 44 & 1 & $5.8 \pm 0.7$ & $17.0 \pm 1.1$ & 46.0 & 41.5 & 144 \\
\hline 45 & 1 & $20.0 \pm 2.2$ & $23.1 \pm 2.0$ & 27.3 & 24.9 & 167 \\
\hline 46 & 1 & $178 \pm 4$ & $179 \pm 4$ & 24.9 & 24.9 & 146 \\
\hline
\end{tabular}


Table A.6. Same as Table A.1 for Herschel-500 $\mu \mathrm{m}$

\begin{tabular}{|c|c|c|c|c|c|c|}
\hline $\begin{array}{l}\text { Number } \\
\text { Id }\end{array}$ & $\operatorname{Tag}_{500}$ & $\begin{array}{c}S_{500}^{\text {peak }} \\
\text { (Jy/beam) }\end{array}$ & $\begin{array}{l}S_{500}^{\text {int }} \\
(\mathrm{Jy})\end{array}$ & $\begin{array}{c}F W H M_{500}^{\text {maj }} \\
(\operatorname{arcsec})\end{array}$ & $\begin{array}{c}F W H M_{500}^{\min } \\
(\operatorname{arcsec})\end{array}$ & $\begin{array}{c}\mathrm{PA}_{500} \\
\text { (degrees) }\end{array}$ \\
\hline 1 & 1 & $548 \pm 4$ & $526 \pm 4$ & 36.3 & 36.3 & 44 \\
\hline 2 & 1 & $310 \pm 5$ & $299 \pm 4$ & 36.3 & 36.3 & 48 \\
\hline 3 & 1 & $205 \pm 5$ & $193 \pm 5$ & 36.3 & 36.3 & 164 \\
\hline 4 & 1 & $67.6 \pm 5.0$ & $64.6 \pm 4.6$ & 36.3 & 36.3 & 161 \\
\hline 5 & 1 & $69.6 \pm 5.2$ & $65.6 \pm 4.7$ & 36.3 & 36.3 & 28 \\
\hline 6 & 1 & $226 \pm 2$ & $209 \pm 1$ & 36.3 & 36.3 & 169 \\
\hline 7 & 1 & $142 \pm 2$ & $134 \pm 1$ & 36.3 & 36.3 & 116 \\
\hline 8 & 1 & $19.2 \pm 5.0$ & $18.1 \pm 4.6$ & 36.3 & 36.3 & 9 \\
\hline 9 & 1 & $53.7 \pm 1.3$ & $52.3 \pm 1.2$ & 36.3 & 36.3 & 172 \\
\hline 10 & 1 & $93.0 \pm 1.2$ & $86.0 \pm 1.1$ & 36.3 & 36.3 & 39 \\
\hline 11 & 1 & $10.4 \pm 1.6$ & $17.2 \pm 1.9$ & 48.2 & 39.0 & 58 \\
\hline 12 & 1 & $75.5 \pm 1.3$ & $74.9 \pm 1.2$ & 36.3 & 36.3 & 60 \\
\hline 13 & 1 & $7.1 \pm 1.9$ & $8.3 \pm 1.7$ & 44.2 & 36.3 & 79 \\
\hline 14 & 1 & $111 \pm 5$ & $105 \pm 4$ & 36.3 & 36.3 & 147 \\
\hline 15 & 1 & $30.5 \pm 1.6$ & $29.0 \pm 1.4$ & 36.3 & 36.3 & 107 \\
\hline 16 & 1 & $9.4 \pm 1.8$ & $9.5 \pm 1.6$ & 36.4 & 36.3 & 87 \\
\hline 17 & 1 & $41.2 \pm 1.5$ & $45.9 \pm 1.3$ & 38.3 & 36.3 & 64 \\
\hline 18 & 1 & $6.4 \pm 1.1$ & $9.2 \pm 1.1$ & 53.1 & 36.3 & 70 \\
\hline 19 & 2 & $8.0 \pm 17.8$ & $7.8 \pm 16.3$ & 36.3 & 36.3 & 80 \\
\hline 20 & 1 & $72.0 \pm 1.3$ & $69.6 \pm 1.2$ & 36.3 & 36.3 & 152 \\
\hline 21 & 1 & $19.6 \pm 0.6$ & $24.8 \pm 0.6$ & 41.9 & 36.3 & 95 \\
\hline 22 & 1 & $22.9 \pm 1.7$ & $23.5 \pm 1.8$ & 36.3 & 36.3 & 33 \\
\hline 23 & 1 & $34.8 \pm 1.7$ & $33.1 \pm 1.5$ & 36.3 & 36.3 & 50 \\
\hline 24 & 1 & $15.8 \pm 0.9$ & $24.7 \pm 1.1$ & 45.2 & 38.3 & 65 \\
\hline 25 & 1 & $37.5 \pm 1.3$ & $35.2 \pm 1.2$ & 36.3 & 36.3 & 93 \\
\hline 26 & 1 & $43.2 \pm 5.0$ & $54.0 \pm 4.6$ & 43.2 & 36.3 & 170 \\
\hline 27 & 1 & $22.9 \pm 1.4$ & $32.0 \pm 1.3$ & 51.1 & 36.3 & 154 \\
\hline 28 & 1 & $40.2 \pm 5.0$ & $38.5 \pm 4.6$ & 36.3 & 36.3 & 150 \\
\hline 29 & 1 & $26.4 \pm 1.5$ & $24.7 \pm 1.4$ & 36.3 & 36.3 & 110 \\
\hline 30 & 1 & $17.2 \pm 0.7$ & $21.3 \pm 0.7$ & 44.0 & 36.3 & 89 \\
\hline 31 & 1 & $6.6 \pm 1.4$ & $7.1 \pm 1.3$ & 36.3 & 36.3 & 91 \\
\hline 32 & 1 & $74.5 \pm 1.3$ & $70.2 \pm 1.1$ & 36.3 & 36.3 & 97 \\
\hline 33 & 1 & $12.8 \pm 0.9$ & $13.0 \pm 0.8$ & 36.3 & 36.3 & 72 \\
\hline 34 & 1 & $9.8 \pm 1.1$ & $9.1 \pm 1.1$ & 37.5 & 36.3 & 59 \\
\hline 35 & 1 & $10.1 \pm 1.0$ & $15.9 \pm 1.1$ & 47.2 & 40.7 & 159 \\
\hline 36 & 1 & $38.8 \pm 1.0$ & $44.2 \pm 0.9$ & 36.6 & 36.3 & 159 \\
\hline 37 & 1 & $5.9 \pm 1.2$ & $7.0 \pm 1.1$ & 48.9 & 36.3 & 106 \\
\hline 38 & 1 & $55.0 \pm 1.3$ & $51.6 \pm 1.2$ & 36.3 & 36.3 & 105 \\
\hline 39 & 1 & $4.5 \pm 0.7$ & $6.2 \pm 0.6$ & 42.0 & 38.9 & 161 \\
\hline 40 & 1 & $22.5 \pm 1.1$ & $23.0 \pm 1.0$ & 36.3 & 36.3 & 57 \\
\hline 41 & 1 & $73.2 \pm 1.2$ & $67.5 \pm 1.1$ & 36.3 & 36.3 & 27 \\
\hline 42 & 1 & $7.1 \pm 0.7$ & $8.9 \pm 0.7$ & 40.5 & 36.3 & 74 \\
\hline 43 & 1 & $28.3 \pm 4.9$ & $31.5 \pm 4.5$ & 41.3 & 36.3 & 13 \\
\hline 44 & 1 & $4.8 \pm 0.9$ & $7.3 \pm 1.0$ & 44.2 & 43.0 & 4 \\
\hline 45 & 1 & $10.7 \pm 1.4$ & $11.0 \pm 1.3$ & 36.3 & 36.3 & 175 \\
\hline 46 & 1 & $71.7 \pm 1.3$ & $69.4 \pm 1.1$ & 36.3 & 36.3 & 130 \\
\hline
\end{tabular}


J. Tigé et al.: The earliest phases of high-mass star formation in NGC 6334

Table A.7. HOBYS catalog of additional GLIMPSE fluxes at 3.6, 4.5, 5.8 and 8.0 $\mu \mathrm{m}$ for the 46 MDCs found in NGC 6334.

\begin{tabular}{|c|c|c|c|c|c|}
\hline & \multicolumn{5}{|c|}{ GLIMPSE } \\
\hline $\begin{array}{c}\text { Number } \\
\text { Id }\end{array}$ & GLIMPSE ID & $\begin{array}{l}S_{3.6}^{\text {int }} \\
(\mathrm{Jy})\end{array}$ & $\begin{array}{l}S_{4.5}^{\text {int }} \\
(\mathrm{Jy})\end{array}$ & $\begin{array}{l}S_{5.8}^{\text {int }} \\
(\mathrm{Jy})\end{array}$ & $\begin{array}{l}S_{8.0}^{\text {int }} \\
(\mathrm{Jy})\end{array}$ \\
\hline 1 & & $0.89^{*}$ & $1.85^{*}$ & $7.7^{*}$ & $39.0^{*}$ \\
\hline 2 & & & & & \\
\hline 3 & & & & & \\
\hline 4 & & & & & \\
\hline 5 & & & & & \\
\hline 6 & & $0.43^{*}$ & $0.85^{*}$ & $0.79^{*}$ & $0.7^{*}$ \\
\hline 7 & & & & & \\
\hline 8 & & & & & \\
\hline 9 & & & & & \\
\hline 10 & & $0.07^{*}$ & $0.12^{*}$ & $1.1^{*}$ & $2.7^{*}$ \\
\hline 11 & & & & & \\
\hline 12 & & & & & \\
\hline 13 & & & & & \\
\hline 14 & & & & & \\
\hline 15 & & & & & \\
\hline 16 & & & & & \\
\hline 17 & & & & & \\
\hline 18 & G352.1150+00.7366 & 0.002 & 0.006 & 0.01 & 0.01 \\
\hline 19 & & & & & \\
\hline 20 & & & & & \\
\hline 21 & & & & & \\
\hline 22 & & & & & \\
\hline 23 & & & & & \\
\hline 24 & G350.8518+00.8209+ G350.8504+00.8198 & 0.003 & 0.007 & 0.015 & 0.02 \\
\hline 25 & & & & & \\
\hline 26 & & & & & \\
\hline 27 & & & & & \\
\hline 28 & & & & & \\
\hline 29 & G351.1556+00.7030 & & & & 0.02 \\
\hline 30 & & & & & \\
\hline 31 & & & & & \\
\hline 32 & & $0.04^{*}$ & $0.22^{*}$ & $0.87^{*}$ & $2.7^{*}$ \\
\hline 33 & & & & & \\
\hline 34 & & & & & \\
\hline 35 & & & & & \\
\hline 36 & & & & & \\
\hline 37 & G350.7304+00.9294 & $3.0 \mathrm{E}-4$ & 0.0015 & 0.005 & 0.007 \\
\hline 38 & & $0.015^{*}$ & $0.05^{*}$ & $0.3^{*}$ & $1.1^{*}$ \\
\hline 39 & & & & & \\
\hline 40 & & & & & \\
\hline 41 & & & & & \\
\hline 42 & & & & & \\
\hline 43 & & & & & \\
\hline 44 & & & & & \\
\hline 45 & & & & & \\
\hline 46 & & $0.22^{*}$ & $1.0^{*}$ & $3.9^{*}$ & $2.7^{*}$ \\
\hline
\end{tabular}

Notes. Stars $\left(^{*}\right)$ on fluxes indicate when we did aperture photometry. 
A\&A 602, A77 (2017)

Table A.8. HOBYS catalog of additional fluxes at Spitzer- $24 \mu \mathrm{m}$, WISE- $22 \mu \mathrm{m}$ or MSX-21 $\mu \mathrm{m}$ for the 46 MDCs found in NGC 6334.

\begin{tabular}{|c|c|c|c|c|c|c|}
\hline & \multicolumn{2}{|l|}{ MIPSGAL } & \multicolumn{2}{|l|}{ WISE } & \multicolumn{2}{|l|}{$M S X$} \\
\hline $\begin{array}{c}\text { Number } \\
\text { Id }\end{array}$ & MIPSGAL ID & $\begin{array}{l}S_{24}^{\text {int }} \\
(\mathrm{Jy})\end{array}$ & WISE ID & $\begin{array}{l}S_{22}^{\text {int }} \\
(\mathrm{Jy})\end{array}$ & $M S X$ ID & $\begin{array}{l}S_{21}^{\text {int }} \\
(\mathrm{Jy})\end{array}$ \\
\hline 1 & saturated & & & & MSX6C G351.4170+00.6440 & 821.0 \\
\hline 2 & & & & & & \\
\hline 3 & & & & & & \\
\hline 4 & & & & & & \\
\hline 5 & & & & & & \\
\hline 6 & saturated & & & & MSX6C G351.1612+00.6973 & 348.0 \\
\hline 7 & saturated & & & & & \\
\hline 8 & & & & & & \\
\hline 9 & & & & & & \\
\hline 10 & saturated & & & & MSX6C G351.2434+00.6664 & 90.0 \\
\hline 11 & & & & & & \\
\hline 12 & & & & & & \\
\hline 13 & & & & & & \\
\hline 14 & MG351.4449+00.6597 & 0.72 & & & & \\
\hline 15 & & & & & & \\
\hline 16 & & & & & & \\
\hline 17 & & & & & & \\
\hline 18 & MG352.1151+00.7366 & 0.03 & & & & \\
\hline 19 & & & & & & \\
\hline 20 & & & & & & \\
\hline 21 & & & & & & \\
\hline 22 & saturated & & & & & \\
\hline 23 & saturated & & & & & \\
\hline 24 & & $0.027^{*}$ & & & & \\
\hline 25 & saturated & & & & & \\
\hline 26 & & & & & & \\
\hline 27 & & & & & & \\
\hline 28 & & & & & & \\
\hline 29 & & & & & & \\
\hline 30 & & & & & & \\
\hline 31 & & & & & & \\
\hline 32 & saturated & & & & MSX6C G351.2434+00.6664 & 90.0 \\
\hline 33 & & & & & & \\
\hline 34 & & & & & & \\
\hline 35 & & & & & & \\
\hline 36 & & & & & & \\
\hline 37 & MG350.7303+00.9294 & 0.07 & & & & \\
\hline 38 & saturated & & & & MSX6C G351.2514+00.6731 & 30.0 \\
\hline 39 & & & & & & \\
\hline 40 & & & & & & \\
\hline 41 & saturated & & & & & \\
\hline 42 & & & & & & \\
\hline 43 & & & & & & \\
\hline 44 & & & & & & \\
\hline 45 & & & & & & \\
\hline 46 & & & $\mathrm{~J} 172023.54-355500.2$ & 22.0 & & \\
\hline
\end{tabular}

Notes. Stars $\left(^{*}\right)$ on fluxes indicate when we did aperture photometry. 
J. Tigé et al.: The earliest phases of high-mass star formation in NGC 6334

Table A.9. Same as Table A.1 for SCUBA2-450 $\mu \mathrm{m}$.

\begin{tabular}{|c|c|c|c|c|c|c|}
\hline $\begin{array}{c}\text { Number } \\
\text { Id }\end{array}$ & $\mathrm{Tag}_{450}$ & $\begin{array}{c}S_{450}^{\text {peak }} \\
\text { (Jy/beam) }\end{array}$ & $\begin{array}{l}S_{450}^{\text {int }} \\
(\mathrm{Jy})\end{array}$ & $\begin{array}{c}F W H M_{450}^{\mathrm{maj}} \\
(\operatorname{arcsec})\end{array}$ & $\begin{array}{c}F W H M_{450}^{\min } \\
(\operatorname{arcsec})\end{array}$ & $\begin{array}{c}\mathrm{PA}_{450} \\
\text { (degrees) }\end{array}$ \\
\hline 1 & 1 & $69.9 \pm 3.2$ & $411 \pm 7$ & 19.4 & 17.6 & 101 \\
\hline 2 & 1 & $21.8 \pm 9.0$ & $93.9 \pm 15.2$ & 19.4 & 12.8 & 179 \\
\hline 3 & 2 & $5.5 \pm 9.5$ & $12.8 \pm 10.0$ & 14.1 & 8.5 & 46 \\
\hline 4 & 2 & $3.2 \pm 7.4$ & $5.0 \pm 7.4$ & 10.5 & 8.8 & 54 \\
\hline 5 & 1 & $7.6 \pm 0.3$ & $25.3 \pm 0.6$ & 17.3 & 10.3 & 14 \\
\hline 6 & 0 & & & & & \\
\hline 7 & 0 & & & & & \\
\hline 8 & 2 & $1.5 \pm 3.8$ & $2.9 \pm 3.8$ & 15.4 & 8.5 & 104 \\
\hline 9 & 0 & & & & & \\
\hline 10 & 0 & & & & & \\
\hline 11 & 0 & & & & & \\
\hline 12 & 0 & & & & & \\
\hline 13 & 0 & & & & & \\
\hline 14 & 1 & $27.2 \pm 8.7$ & $54.4 \pm 9.5$ & 14.6 & 9.8 & 165 \\
\hline 15 & 0 & & & & & \\
\hline 16 & 0 & & & & & \\
\hline 17 & 0 & & & & & \\
\hline 18 & 0 & & & & & \\
\hline 19 & 0 & & & & & \\
\hline 20 & 0 & & & & & \\
\hline 21 & 0 & & & & & \\
\hline 22 & 0 & & & & & \\
\hline 23 & 0 & & & & & \\
\hline 24 & 0 & & & & & \\
\hline 25 & 0 & & & & & \\
\hline 26 & 2 & $0.31 \pm 3.0$ & $-7.8 \pm 3.0$ & 8.5 & 8.5 & 0 \\
\hline 27 & 0 & & & & & \\
\hline 28 & 1 & $7.9 \pm 2.1$ & $19.6 \pm 3.8$ & 15.2 & 8.5 & 48 \\
\hline 29 & 0 & & & & & \\
\hline 30 & 0 & & & & & \\
\hline 31 & 0 & & & & & \\
\hline 32 & 0 & & & & & \\
\hline 33 & 0 & & & & & \\
\hline 34 & 0 & & & & & \\
\hline 35 & 0 & & & & & \\
\hline 36 & 0 & & & & & \\
\hline 37 & 0 & & & & & \\
\hline 38 & 0 & & & & & \\
\hline 39 & 0 & & & & & \\
\hline 40 & 0 & & & & & \\
\hline 41 & 0 & & & & & \\
\hline 42 & 0 & & & & & \\
\hline 43 & 1 & $1.6 \pm 0.6$ & $4.2 \pm 0.6$ & 11.9 & 9.6 & 175 \\
\hline 44 & 0 & & & & & \\
\hline 45 & 0 & & & & & \\
\hline 46 & 0 & & & & & \\
\hline
\end{tabular}


A\&A 602, A77 (2017)

Table A.10. Same as Table A.1 for SCUBA2-850 $\mu \mathrm{m}$

\begin{tabular}{|c|c|c|c|c|c|c|}
\hline $\begin{array}{l}\text { Number } \\
\text { Id }\end{array}$ & $\mathrm{Tag}_{850}$ & $\begin{array}{c}S_{850}^{\text {peak }} \\
\text { (Jy/beam) }\end{array}$ & $\begin{array}{l}S_{850}^{\text {int }} \\
(\mathrm{Jy})\end{array}$ & $\begin{array}{c}F W H M_{850}^{\mathrm{maj}} \\
(\operatorname{arcsec})\end{array}$ & $\begin{array}{c}F W H M_{850}^{\min } \\
(\operatorname{arcsec})\end{array}$ & $\begin{array}{c}\mathrm{PA}_{850} \\
\text { (degrees) }\end{array}$ \\
\hline 1 & 1 & $23.7 \pm 0.5$ & $36.8 \pm 0.8$ & 16.6 & 15.0 & 49 \\
\hline 2 & 1 & $9.2 \pm 0.7$ & $15.4 \pm 0.7$ & 22.7 & 15.0 & 171 \\
\hline 3 & 1 & $8.1 \pm 0.6$ & $12.5 \pm 0.6$ & 18.8 & 15.0 & 71 \\
\hline 4 & 1 & $1.7 \pm 0.5$ & $2.2 \pm 0.5$ & 19.1 & 15.0 & 17 \\
\hline 5 & 1 & $1.8 \pm 0.5$ & $2.5 \pm 0.7$ & 16.9 & 15.0 & 57 \\
\hline 6 & 0 & & & & & \\
\hline 7 & 0 & & & & & \\
\hline 8 & 1 & $1.3 \pm 0.6$ & $2.3 \pm 0.6$ & 21.2 & 15 & 159 \\
\hline 9 & 0 & & & & & \\
\hline 10 & 0 & & & & & \\
\hline 11 & 0 & & & & & \\
\hline 12 & 0 & & & & & \\
\hline 13 & 0 & & & & & \\
\hline 14 & 1 & $7.6 \pm 0.6$ & $8.7 \pm 0.6$ & 15.1 & 15.0 & 148 \\
\hline 15 & 0 & & & & & \\
\hline 16 & 0 & & & & & \\
\hline 17 & 0 & & & & & \\
\hline 18 & 0 & & & & & \\
\hline 19 & 0 & & & & & \\
\hline 20 & 0 & & & & & \\
\hline 21 & 0 & & & & & \\
\hline 22 & 0 & & & & & \\
\hline 23 & 0 & & & & & \\
\hline 24 & 0 & & & & & \\
\hline 25 & 0 & & & & & \\
\hline 26 & 2 & $0.49 \pm 0.63$ & $0.50 \pm 0.63$ & 15 & 15 & 167 \\
\hline 27 & 0 & & & & & \\
\hline 28 & 2 & $1.7 \pm 0.8$ & $1.7 \pm 1.0$ & 18.7 & 15.0 & 52 \\
\hline 29 & 0 & & & & & \\
\hline 30 & 0 & & & & & \\
\hline 31 & 0 & & & & & \\
\hline 32 & 0 & & & & & \\
\hline 33 & 0 & & & & & \\
\hline 34 & 0 & & & & & \\
\hline 35 & 0 & & & & & \\
\hline 36 & 0 & & & & & \\
\hline 37 & 0 & & & & & \\
\hline 38 & 0 & & & & & \\
\hline 39 & 0 & & & & & \\
\hline 40 & 0 & & & & & \\
\hline 41 & 0 & & & & & \\
\hline 42 & 0 & & & & & \\
\hline 43 & 1 & $0.79 \pm 0.17$ & $0.79 \pm 0.17$ & 15.0 & 15.0 & 13 \\
\hline 44 & 0 & & & & & \\
\hline 45 & 0 & & & & & \\
\hline 46 & 0 & & & & & \\
\hline
\end{tabular}


Table A.11. Same as Table A.1 for APEX-870 $\mu \mathrm{m}$

\begin{tabular}{|c|c|c|c|c|c|c|}
\hline $\begin{array}{l}\text { Number } \\
\text { Id }\end{array}$ & $\operatorname{Tag}_{870}$ & $\begin{array}{c}S_{870}^{\text {peak }} \\
\text { (Jy/beam) }\end{array}$ & $\begin{array}{l}S_{870}^{\text {int }} \\
(\mathrm{Jy})\end{array}$ & $\begin{array}{c}F W H M_{870}^{\text {maj }} \\
(\operatorname{arcsec})\end{array}$ & $\begin{array}{c}F W H M_{870}^{\min } \\
(\operatorname{arcsec})\end{array}$ & $\begin{array}{c}\mathrm{PA}_{870} \\
\text { (degrees) }\end{array}$ \\
\hline 1 & 1 & $44.0 \pm 1.1$ & $55.5 \pm 1.3$ & 21.0 & 18.2 & 28 \\
\hline 2 & 1 & $17.4 \pm 1.0$ & $21.2 \pm 1.0$ & 20.2 & 18.2 & 170 \\
\hline 3 & 1 & $11.1 \pm 1.1$ & $12.4 \pm 1.1$ & 22.5 & 18.2 & 61 \\
\hline 4 & 1 & $9.2 \pm 1.1$ & $15.2 \pm 1.1$ & 29.2 & 18.2 & 178 \\
\hline 5 & 1 & $2.7 \pm 0.5$ & $3.9 \pm 0.5$ & 24.1 & 18.2 & 17 \\
\hline 6 & 1 & $18.0 \pm 1.0$ & $22.8 \pm 1.0$ & 18.7 & 18.2 & 118 \\
\hline 7 & 1 & $7.4 \pm 0.9$ & $10.6 \pm 0.9$ & 21.5 & 18.2 & 77 \\
\hline 8 & 0 & & & & & \\
\hline 9 & 1 & $4.4 \pm 0.4$ & $5.7 \pm 0.4$ & 20.0 & 18.2 & 159 \\
\hline 10 & 1 & $9.2 \pm 0.4$ & $15.6 \pm 0.4$ & 22.9 & 21.4 & 124 \\
\hline 11 & 1 & $0.4 \pm 0.12$ & $2.0 \pm 0.2$ & 58.2 & 32.5 & 128 \\
\hline 12 & 1 & $3.3 \pm 0.4$ & $5.3 \pm 0.4$ & 26.0 & 18.2 & 2 \\
\hline 13 & 2 & $0.18 \pm 0.11$ & $0.5 \pm 0.13$ & 38.2 & 21.6 & 72 \\
\hline 14 & 1 & $23.5 \pm 1.1$ & $39.8 \pm 1.6$ & 23.9 & 18.2 & 175 \\
\hline 15 & 1 & $5.2 \pm 0.9$ & $9.8 \pm 0.9$ & 29.7 & 21.2 & 125 \\
\hline 16 & 1 & $0.5 \pm 0.13$ & $0.9 \pm 0.15$ & 26.0 & 19.3 & 10 \\
\hline 17 & 1 & $1.4 \pm 0.5$ & $2.1 \pm 0.5$ & 25.2 & 20.2 & 15 \\
\hline 18 & 1 & $0.4 \pm 0.08$ & $1.9 \pm 0.16$ & 52.4 & 33.2 & 51 \\
\hline 19 & 1 & $4.0 \pm 0.4$ & $6.8 \pm 0.4$ & 27.3 & 18.2 & 3 \\
\hline 20 & 1 & $2.5 \pm 0.4$ & $2.7 \pm 0.4$ & 18.2 & 18.2 & 126 \\
\hline 21 & 1 & $0.6 \pm 0.13$ & $3.6 \pm 0.2$ & 48.6 & 37.6 & 19 \\
\hline 22 & 1 & $1.7 \pm 0.3$ & $2.0 \pm 0.3$ & 20.6 & 18.2 & 39 \\
\hline 23 & 1 & $4.2 \pm 0.3$ & $5.3 \pm 0.3$ & 18.2 & 18.2 & 18 \\
\hline 24 & 1 & $0.9 \pm 0.07$ & $2.9 \pm 0.12$ & 31.1 & 26.7 & 93 \\
\hline 25 & 1 & $3.9 \pm 0.4$ & $4.1 \pm 0.4$ & 18.2 & 18.2 & 116 \\
\hline 26 & 1 & $2.7 \pm 1.1$ & $3.4 \pm 1.1$ & 19.8 & 18.2 & 134 \\
\hline 27 & 1 & $0.7 \pm 0.3$ & $1.1 \pm 0.3$ & 28.5 & 19.5 & 124 \\
\hline 28 & 0 & & & & & \\
\hline 29 & 2 & $1.0 \pm 0.8$ & $3.3 \pm 0.8$ & 31.6 & 27.5 & 112 \\
\hline 30 & 1 & $0.8 \pm 0.13$ & $1.5 \pm 0.16$ & 27.9 & 19.1 & 81 \\
\hline 31 & 2 & $0.3 \pm 0.18$ & $0.6 \pm 0.19$ & 27.9 & 20.3 & 163 \\
\hline 32 & 1 & $6.0 \pm 0.4$ & $6.5 \pm 0.4$ & 18.2 & 18.2 & 51 \\
\hline 33 & 1 & $0.8 \pm 0.12$ & $1.3 \pm 0.12$ & 23.8 & 18.6 & 173 \\
\hline 34 & 2 & $0.3 \pm 0.11$ & $0.4 \pm 0.11$ & 26.0 & 20.4 & 111 \\
\hline 35 & 1 & $0.6 \pm 0.06$ & $4.3 \pm 0.15$ & 50.4 & 44.0 & 101 \\
\hline 36 & 1 & $1.2 \pm 0.3$ & $1.9 \pm 0.3$ & 26.5 & 19.6 & 74 \\
\hline 37 & 1 & $0.6 \pm 0.17$ & $1.1 \pm 0.3$ & 22.6 & 21.4 & 156 \\
\hline 38 & 1 & $10.0 \pm 0.4$ & $11.6 \pm 0.8$ & 18.2 & 18.2 & 4 \\
\hline 39 & 2 & $0.17 \pm 0.07$ & $0.40 \pm 0.09$ & 25.8 & 22.6 & 88 \\
\hline 40 & 1 & $0.6 \pm 0.2$ & $0.7 \pm 0.2$ & 21.4 & 18.2 & 84 \\
\hline 41 & 1 & $6.8 \pm 0.4$ & $7.4 \pm 0.4$ & 18.2 & 18.2 & 163 \\
\hline 42 & 1 & $0.3 \pm 0.09$ & $1.8 \pm 0.16$ & 52.2 & 35.9 & 71 \\
\hline 43 & 2 & $1.7 \pm 0.2$ & $1.2 \pm 0.2$ & 18.2 & 18.2 & 2 \\
\hline 44 & 2 & $0.4 \pm 0.08$ & $0.8 \pm 0.13$ & 47.0 & 22.4 & 6 \\
\hline 45 & 1 & $0.9 \pm 0.15$ & $1.6 \pm 0.19$ & 23.1 & 18.9 & 2 \\
\hline 46 & 1 & $4.5 \pm 0.4$ & $5.7 \pm 0.4$ & 19.7 & 18.2 & 135 \\
\hline
\end{tabular}


Table A.12. Same as Table A.1 for SIMBA- $1200 \mu \mathrm{m}$

\begin{tabular}{|c|c|c|c|c|c|c|}
\hline $\begin{array}{l}\text { Number } \\
\text { Id }\end{array}$ & $\operatorname{Tag}_{1200}$ & $\begin{array}{c}S_{1200}^{\text {peak }} \\
\text { (Jy/beam) }\end{array}$ & $\begin{array}{l}S_{1200}^{\text {int }} \\
(\mathrm{Jy})\end{array}$ & $\begin{array}{c}F W H M_{1200}^{\mathrm{maj}} \\
(\operatorname{arcsec})\end{array}$ & $\begin{array}{c}F W H M_{1200}^{\min } \\
(\operatorname{arcsec})\end{array}$ & $\begin{array}{c}\mathrm{PA}_{1200} \\
\text { (degrees) }\end{array}$ \\
\hline 1 & 1 & $15.1 \pm 0.6$ & $20.2 \pm 0.6$ & 26.8 & 24.0 & 1 \\
\hline 2 & 1 & $7.3 \pm 0.6$ & $9.3 \pm 0.6$ & 24.4 & 24.0 & 26 \\
\hline 3 & 1 & $7.1 \pm 0.6$ & $8.0 \pm 0.6$ & 24.2 & 24.0 & 46 \\
\hline 4 & 1 & $1.8 \pm 0.6$ & $3.2 \pm 0.6$ & 32.6 & 27.6 & 0 \\
\hline 5 & 1 & $0.8 \pm 0.3$ & $0.7 \pm 0.3$ & 24.0 & 24.0 & 34 \\
\hline 6 & 1 & $3.3 \pm 0.13$ & $4.0 \pm 0.13$ & 24.0 & 24.0 & 169 \\
\hline 7 & 1 & $2.8 \pm 0.13$ & $3.1 \pm 0.13$ & 24.0 & 24.0 & 142 \\
\hline 8 & 1 & $2.4 \pm 0.7$ & $3.3 \pm 0.7$ & 27.8 & 24.0 & 151 \\
\hline 9 & 1 & $1.6 \pm 0.1$ & $2.4 \pm 0.1$ & 31.7 & 24.0 & 80 \\
\hline 10 & 1 & $3.5 \pm 0.08$ & $3.8 \pm 0.08$ & 24.0 & 24.0 & 107 \\
\hline 11 & 1 & $0.3 \pm 0.04$ & $1.5 \pm 0.09$ & 51.4 & 45.3 & 96 \\
\hline 12 & 1 & $3.6 \pm 0.09$ & $5.1 \pm 0.09$ & 30.5 & 24.0 & 89 \\
\hline 13 & 1 & $0.13 \pm 0.07$ & $0.2 \pm 0.07$ & 32.2 & 24.0 & 68 \\
\hline 14 & 1 & $2.8 \pm 0.6$ & $3.1 \pm 0.6$ & 24.0 & 24.0 & 136 \\
\hline 15 & 1 & $1.3 \pm 0.13$ & $1.7 \pm 0.13$ & 25.0 & 24.0 & 101 \\
\hline 16 & 1 & $0.17 \pm 0.07$ & $0.3 \pm 0.07$ & 32.5 & 28.1 & 13 \\
\hline 17 & 1 & $0.4 \pm 0.2$ & $0.5 \pm 0.16$ & 29.0 & 24.0 & 8 \\
\hline 18 & 2 & $0.1 \pm 0.05$ & $0.3 \pm 0.06$ & 60.8 & 27.7 & 68 \\
\hline 19 & 1 & $1.0 \pm 0.1$ & $1.1 \pm 0.1$ & 24.0 & 24.0 & 90 \\
\hline 20 & 1 & $0.8 \pm 0.1$ & $1.0 \pm 0.1$ & 24.0 & 24.0 & 98 \\
\hline 21 & 1 & $0.17 \pm 0.04$ & $0.5 \pm 0.06$ & 40.6 & 34.2 & 42 \\
\hline 22 & 1 & $1.4 \pm 0.12$ & $1.6 \pm 0.12$ & 24.0 & 24.0 & 9 \\
\hline 23 & 1 & $1.7 \pm 0.12$ & $1.9 \pm 0.12$ & 24.0 & 24.0 & 48 \\
\hline 24 & 1 & $0.3 \pm 0.03$ & $0.8 \pm 0.04$ & 42.4 & 36.5 & 128 \\
\hline 25 & 1 & $1.1 \pm 0.09$ & $1.6 \pm 0.09$ & 35.1 & 24.0 & 93 \\
\hline 26 & 0 & & & & & \\
\hline 27 & 2 & $0.15 \pm 0.08$ & $0.2 \pm 0.08$ & 33.3 & 24.0 & 159 \\
\hline 28 & 2 & $0.7 \pm 0.5$ & $1.0 \pm 0.5$ & 33.0 & 24.0 & 9 \\
\hline 29 & 1 & $1.7 \pm 0.13$ & $1.8 \pm 0.13$ & 24.0 & 24.0 & 153 \\
\hline 30 & 1 & $0.3 \pm 0.07$ & $0.4 \pm 0.08$ & 41.4 & 24.0 & 91 \\
\hline 31 & 2 & $0.09 \pm 0.07$ & $0.13 \pm 0.07$ & 29.6 & 25.7 & 29 \\
\hline 32 & 1 & $2.6 \pm 0.09$ & $2.8 \pm 0.09$ & 24.0 & 24.0 & 23 \\
\hline 33 & 1 & $0.2 \pm 0.05$ & $0.4 \pm 0.05$ & 33.8 & 28.3 & 134 \\
\hline 34 & 2 & $0.11 \pm 0.09$ & $0.13 \pm 0.09$ & 26.5 & 24.0 & 62 \\
\hline 35 & 1 & $0.18 \pm 0.02$ & $0.5 \pm 0.03$ & 45.7 & 27.3 & 157 \\
\hline 36 & 1 & $0.4 \pm 0.08$ & $0.6 \pm 0.08$ & 30.6 & 24.9 & 165 \\
\hline 37 & 2 & $0.1 \pm 0.07$ & $0.18 \pm 0.08$ & 37.2 & 27.9 & 163 \\
\hline 38 & 1 & $1.2 \pm 0.08$ & $1.9 \pm 0.12$ & 28.9 & 24.0 & 172 \\
\hline 39 & 2 & $0.018 \pm 0.033$ & $0.035 \pm 0.034$ & 31.4 & 24.0 & 103 \\
\hline 40 & 2 & $0.13 \pm 0.09$ & $0.19 \pm 0.09$ & 33.2 & 24.0 & 67 \\
\hline 41 & 1 & $2.6 \pm 0.08$ & $2.8 \pm 0.08$ & 24.0 & 24.0 & 170 \\
\hline 42 & 1 & $0.09 \pm 0.03$ & $0.3 \pm 0.04$ & 47.8 & 31.1 & 91 \\
\hline 43 & 2 & $0.90 \pm 0.04$ & $0.42 \pm 0.04$ & 24.0 & 24.0 & 159 \\
\hline 44 & 1 & $0.07 \pm 0.01$ & $0.18 \pm 0.02$ & 42.2 & 28.4 & 161 \\
\hline 45 & 1 & $0.2 \pm 0.06$ & $0.3 \pm 0.06$ & 30.9 & 24.0 & 172 \\
\hline 46 & 1 & $1.1 \pm 0.1$ & $1.2 \pm 0.1$ & 24.0 & 24.0 & 94 \\
\hline
\end{tabular}


Appendix B: Multi-wavelength images and spectral energy distribution

We present in this appendix the multi-wavelength images and spectral energy distributions (SEDs) for the 46 MDCs of NGC 6334, which are discussed in the main body of the text.

\section{B.1. IR-bright MDCs}
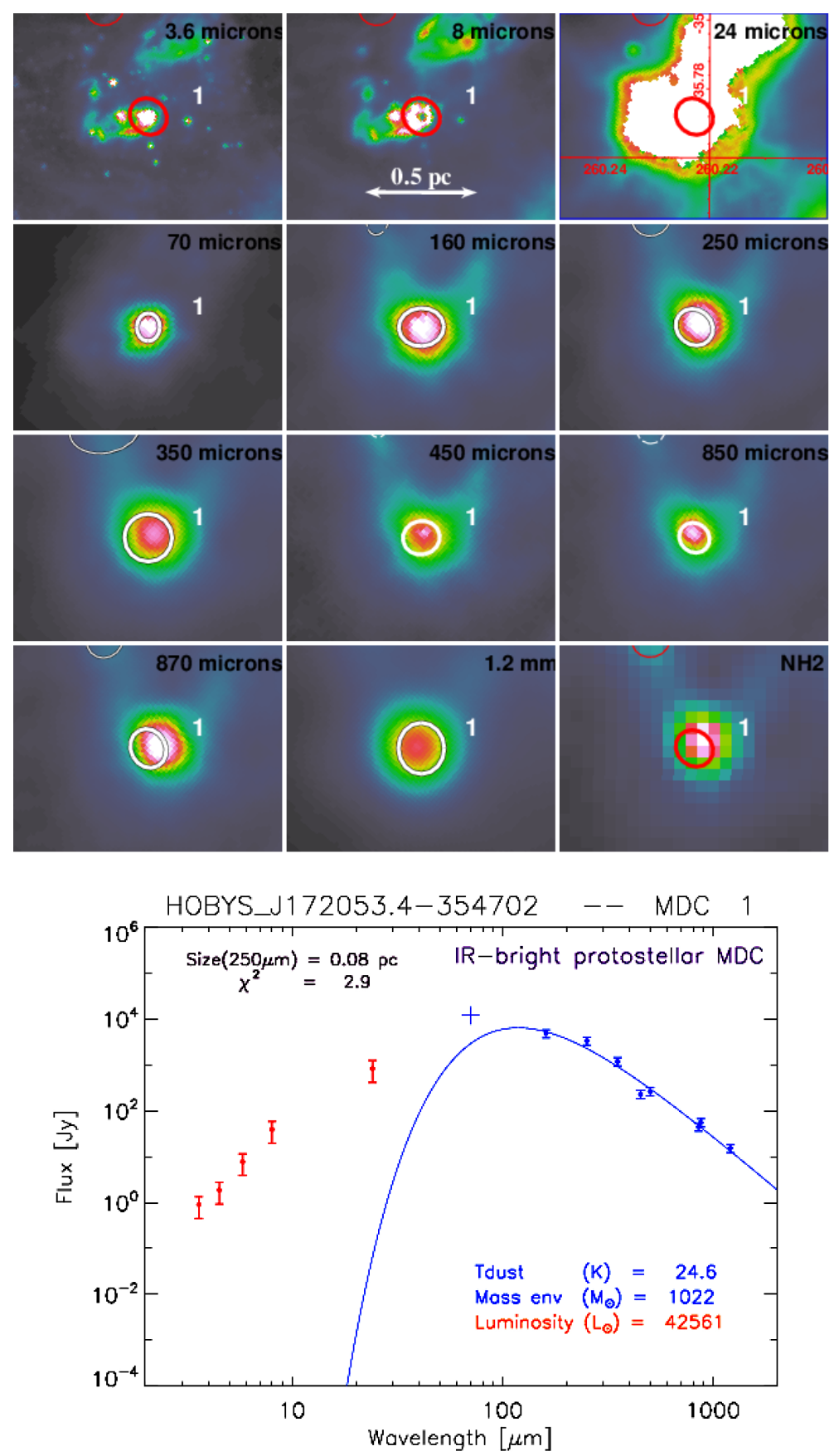

Fig. B.1. Maps are: $3.6 \mu \mathrm{m}, 8 \mu \mathrm{m}, 24 \mu \mathrm{m}, 70 \mu \mathrm{m}, 160 \mu \mathrm{m}, 250 \mu \mathrm{m}, 350 \mu \mathrm{m}, 450 \mu \mathrm{m}, 500 \mu \mathrm{m}, 850 \mu \mathrm{m}, 870 \mu \mathrm{m}, 1.2 \mathrm{~mm}$ and high-resolution $N_{\mathrm{H}_{2}}$ column density. White ellipses represent the getsources FWHM integration size measured for $\geq 70 \mu$ m wavelengths and white numbers are MDC IDs. Red ellipses show the reference FWHM size (at either $160 \mu \mathrm{m}$ or $250 \mu \mathrm{m}$ ), plotted in $<70 \mu \mathrm{m}$ images or images where source flux is not reliable (see Sect. 3.2). Dashed ellipses outline extracted sources, whose fluxes are used as upper limits in SEDs. Linear color scales are adjusted in each image to highlight sources of interest and their close surroundings. Large white areas observed at $24 \mu \mathrm{m}$ toward MDCs \#1, \#6, \#7, \#10, $\# 22$, \#23, \#25, \#32, \#38, and \#41 correspond to regions of flux saturation with Spitzer (see also Table A.8). SED: flux density versus $\lambda$ : blue fit and fluxes are for the MDC envelope and red fluxes are associated IR sources. Crosses at $70 \mu \mathrm{m}$ indicate extracted $70 \mu \mathrm{m}$ fluxes not used in the fitting procedure. 
A\&A 602, A77 (2017)
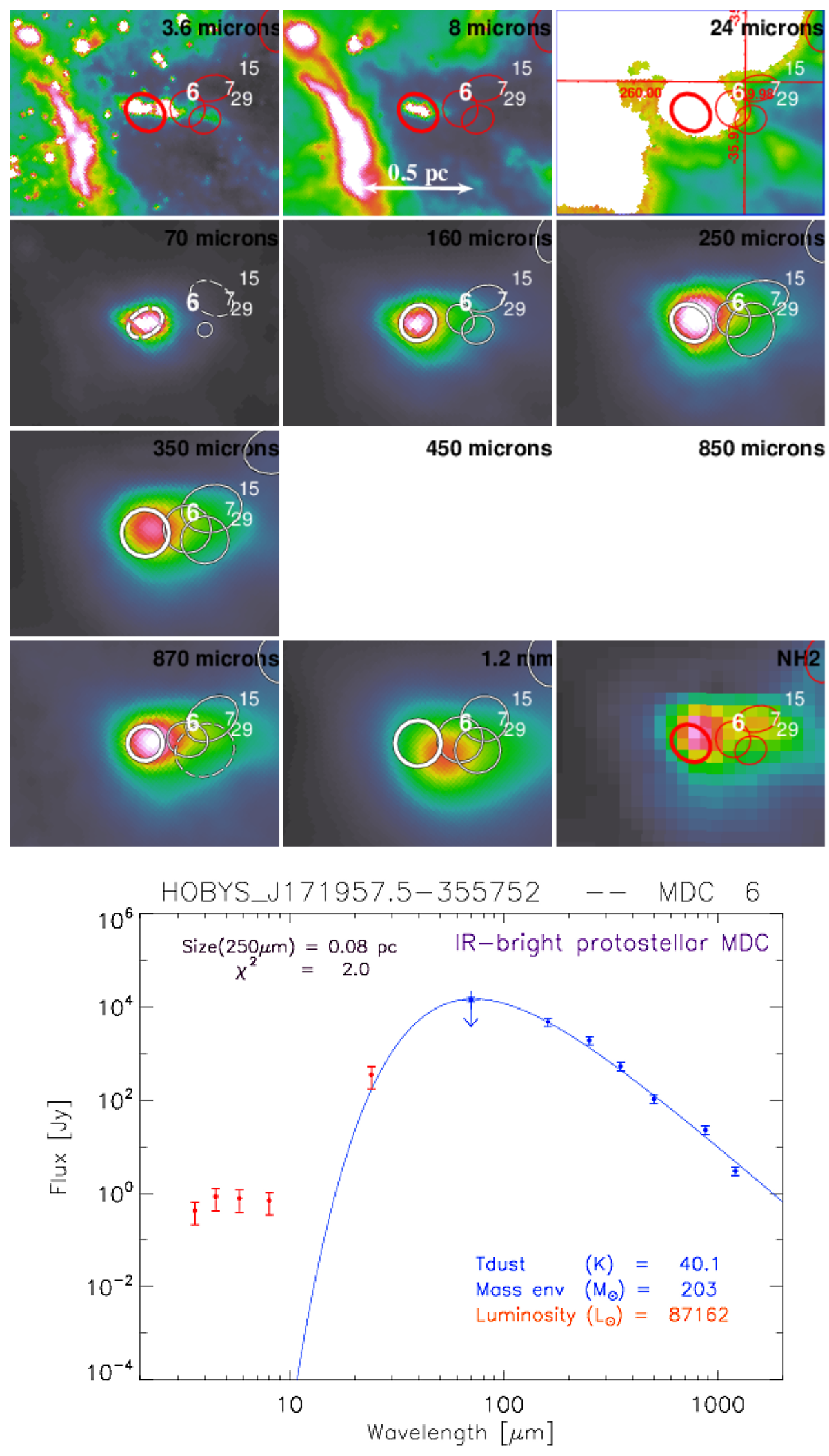

Fig. B.1. continued. 
J. Tigé et al.: The earliest phases of high-mass star formation in NGC 6334
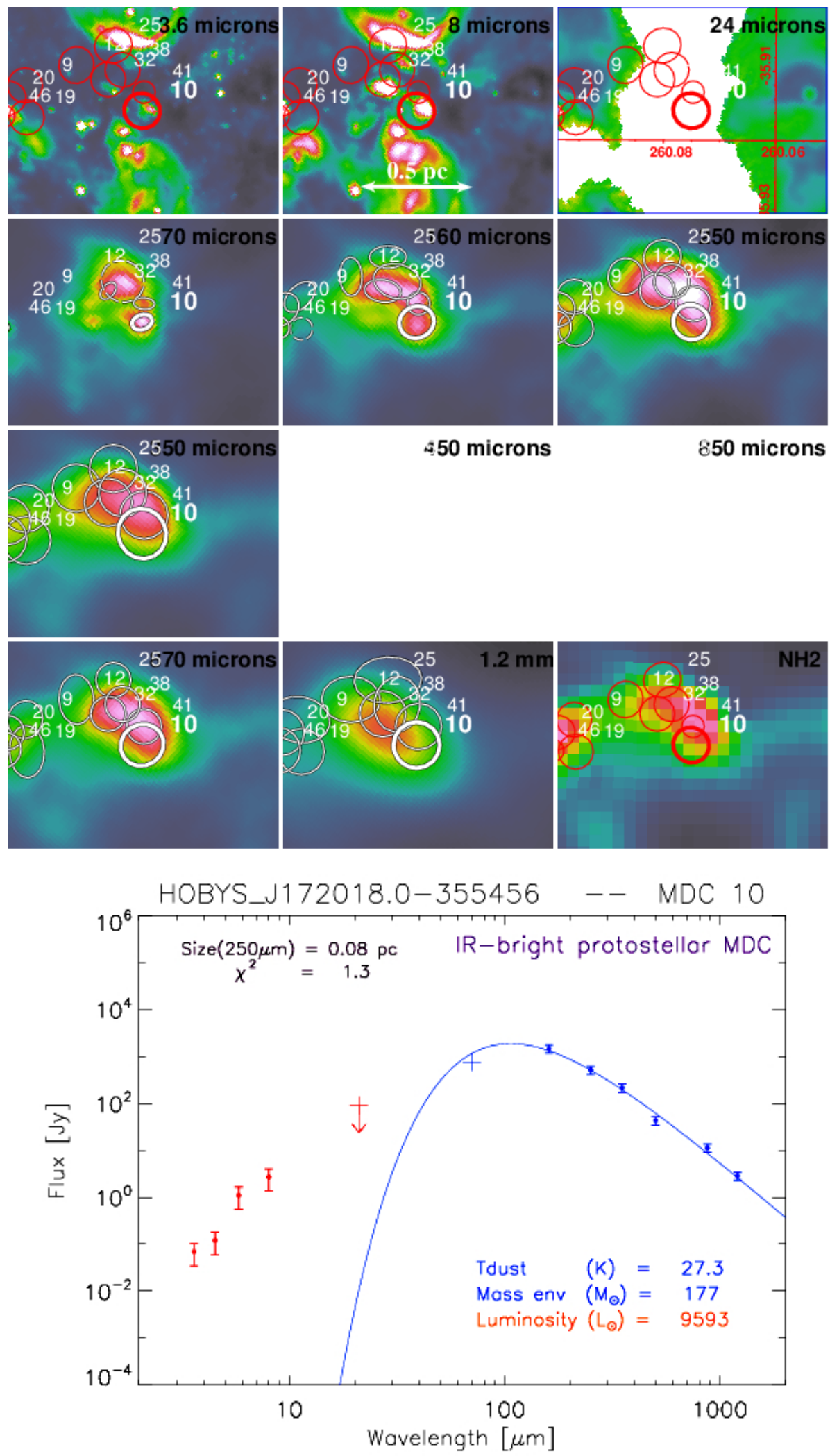

Fig. B.1. continued. 
A\&A 602, A77 (2017)
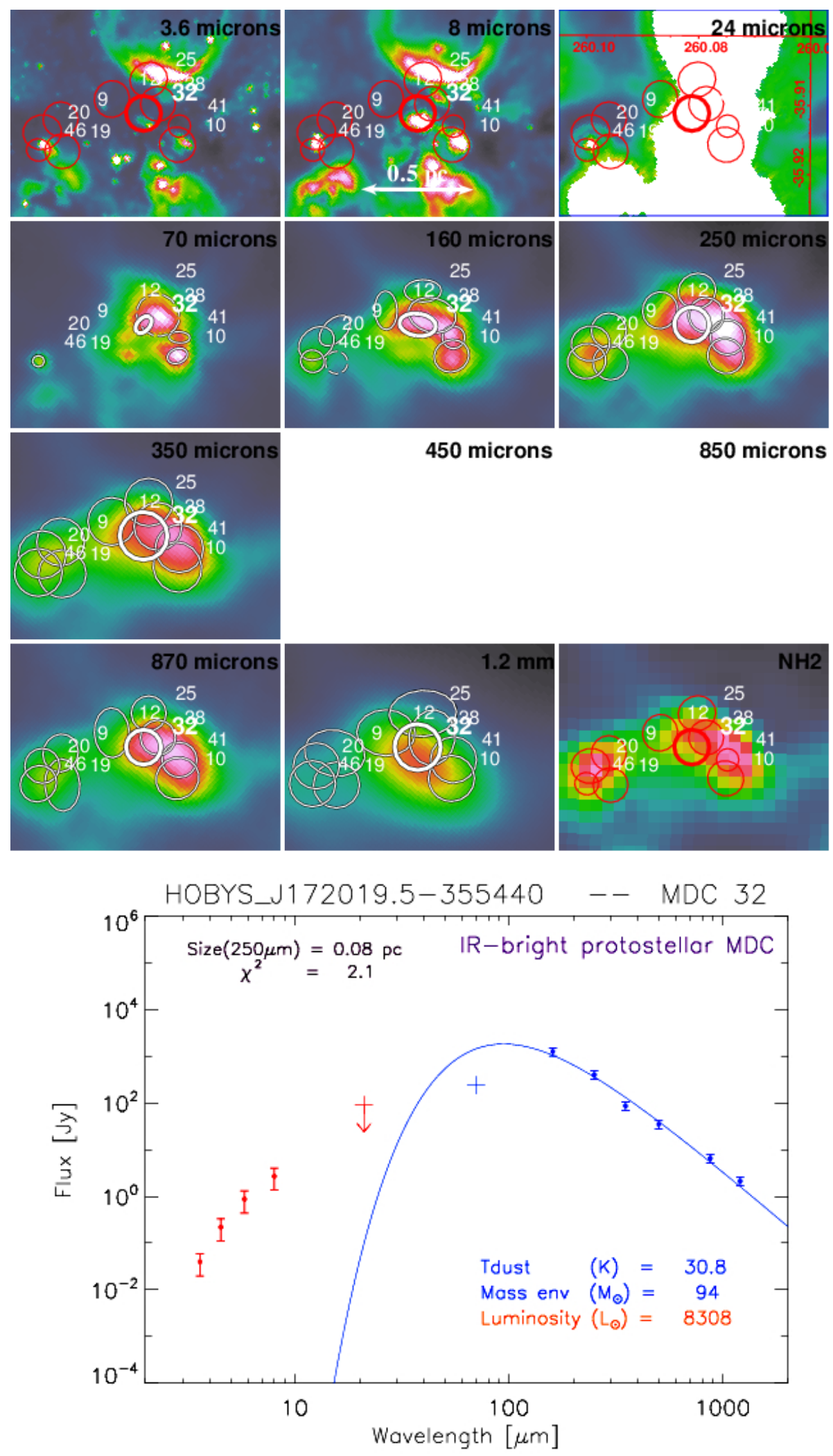

Fig. B.1. continued. 
J. Tigé et al.: The earliest phases of high-mass star formation in NGC 6334
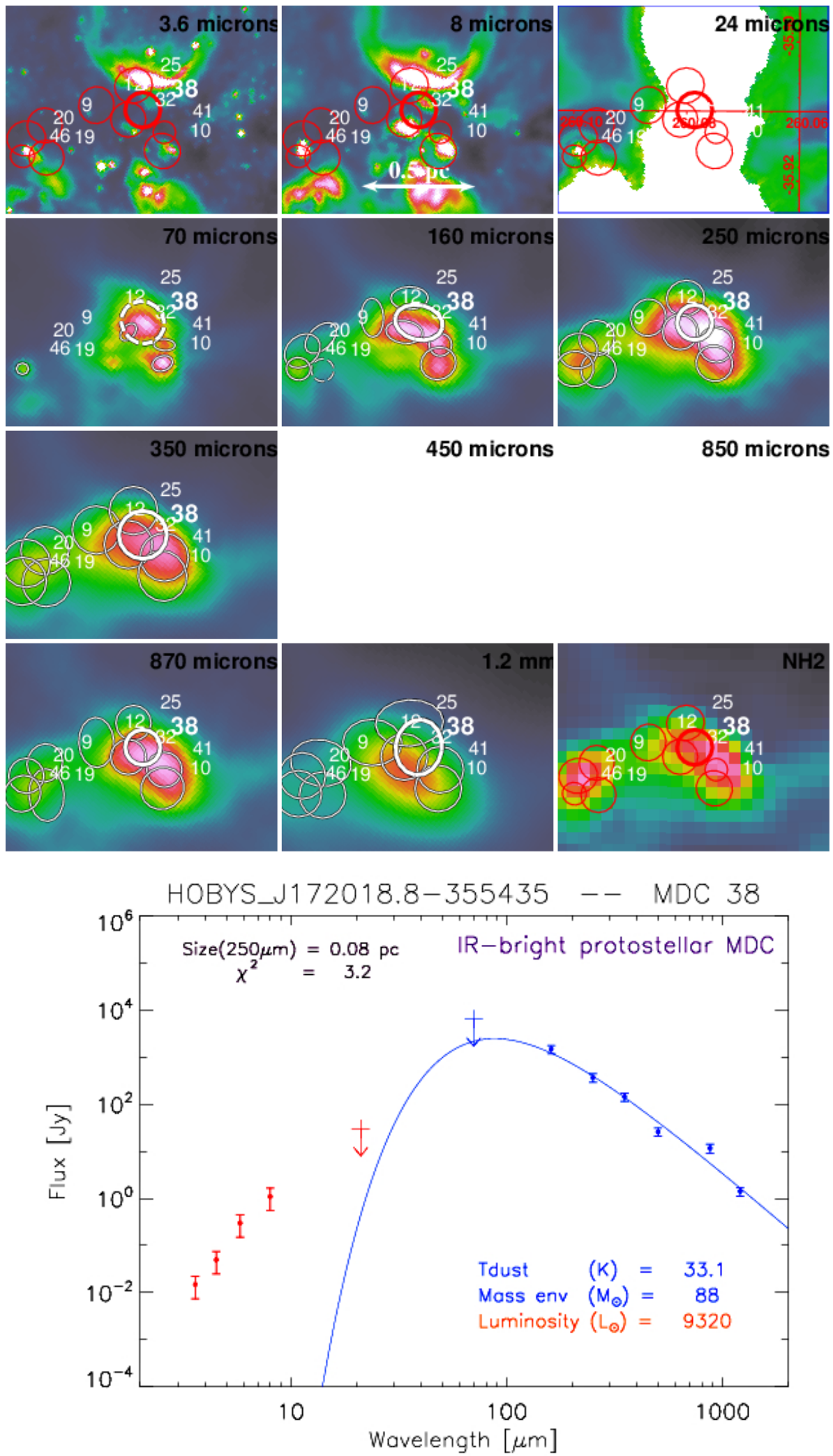

Fig. B.1. continued. 
A\&A 602, A77 (2017)
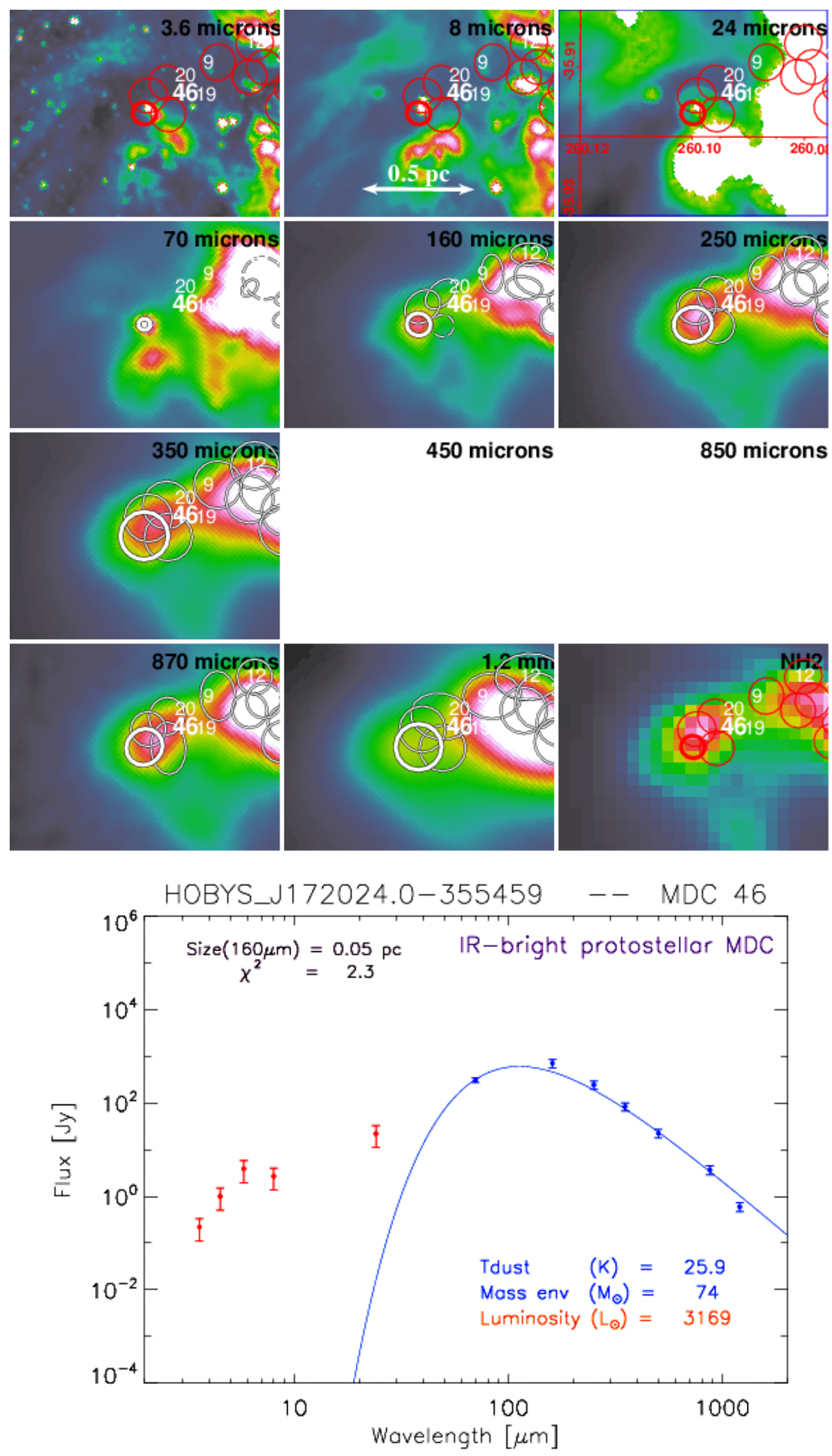

Fig. B.1. continued. 


\section{B.2. IR-quiet $M D C s$}
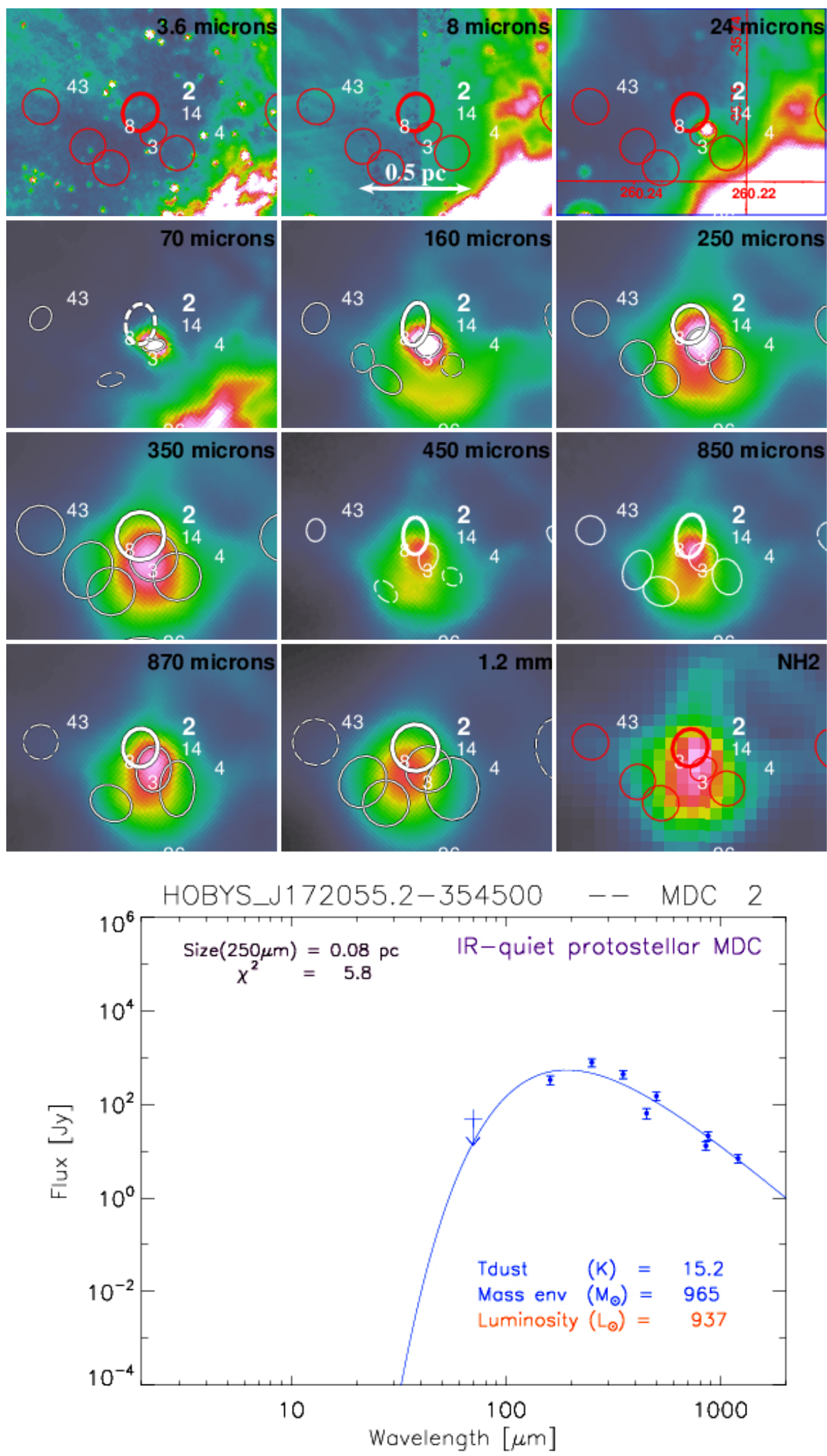

Fig. B.1. continued. 
A\&A 602, A77 (2017)
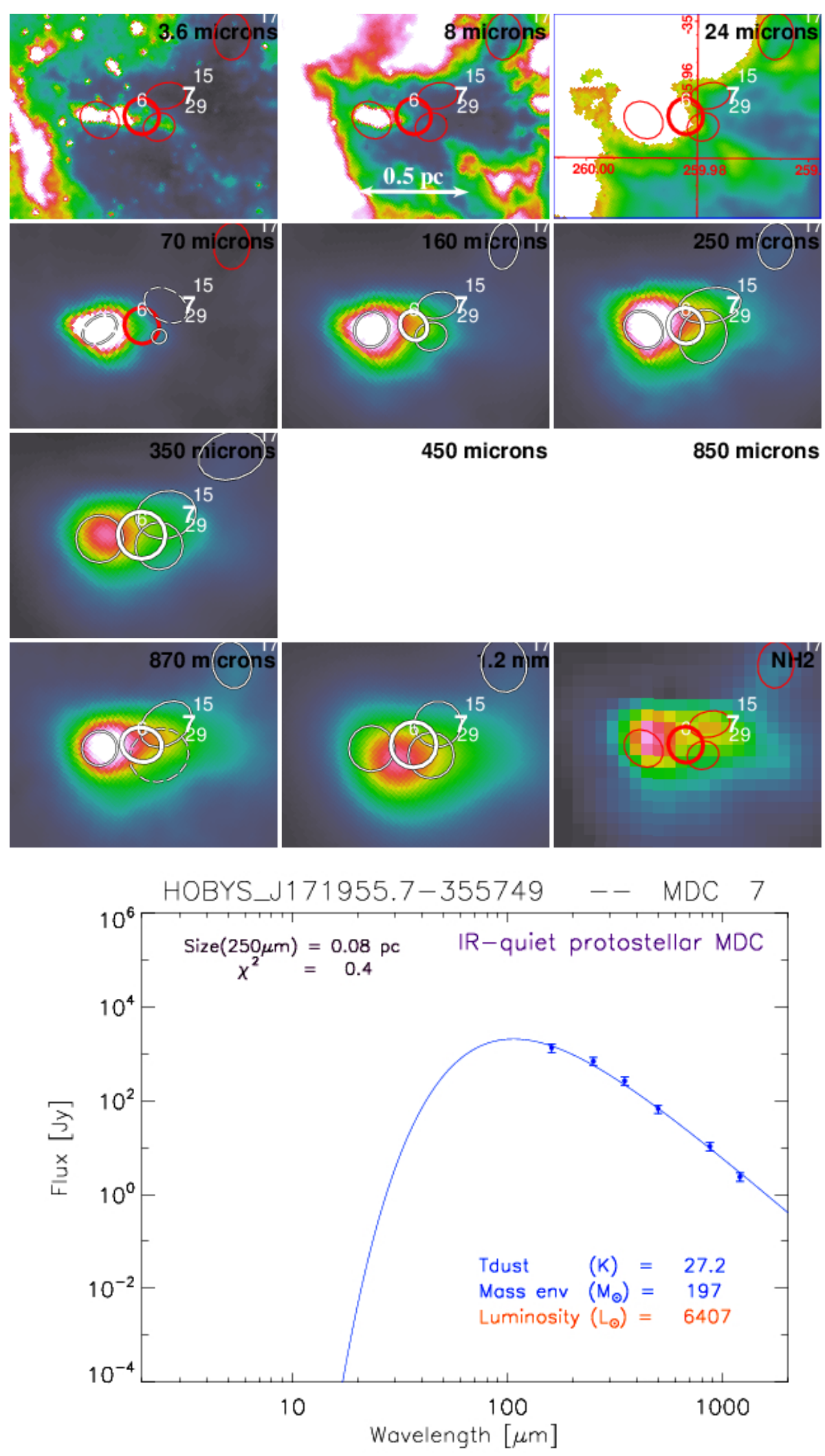

Fig. B.1. continued. 
J. Tigé et al.: The earliest phases of high-mass star formation in NGC 6334
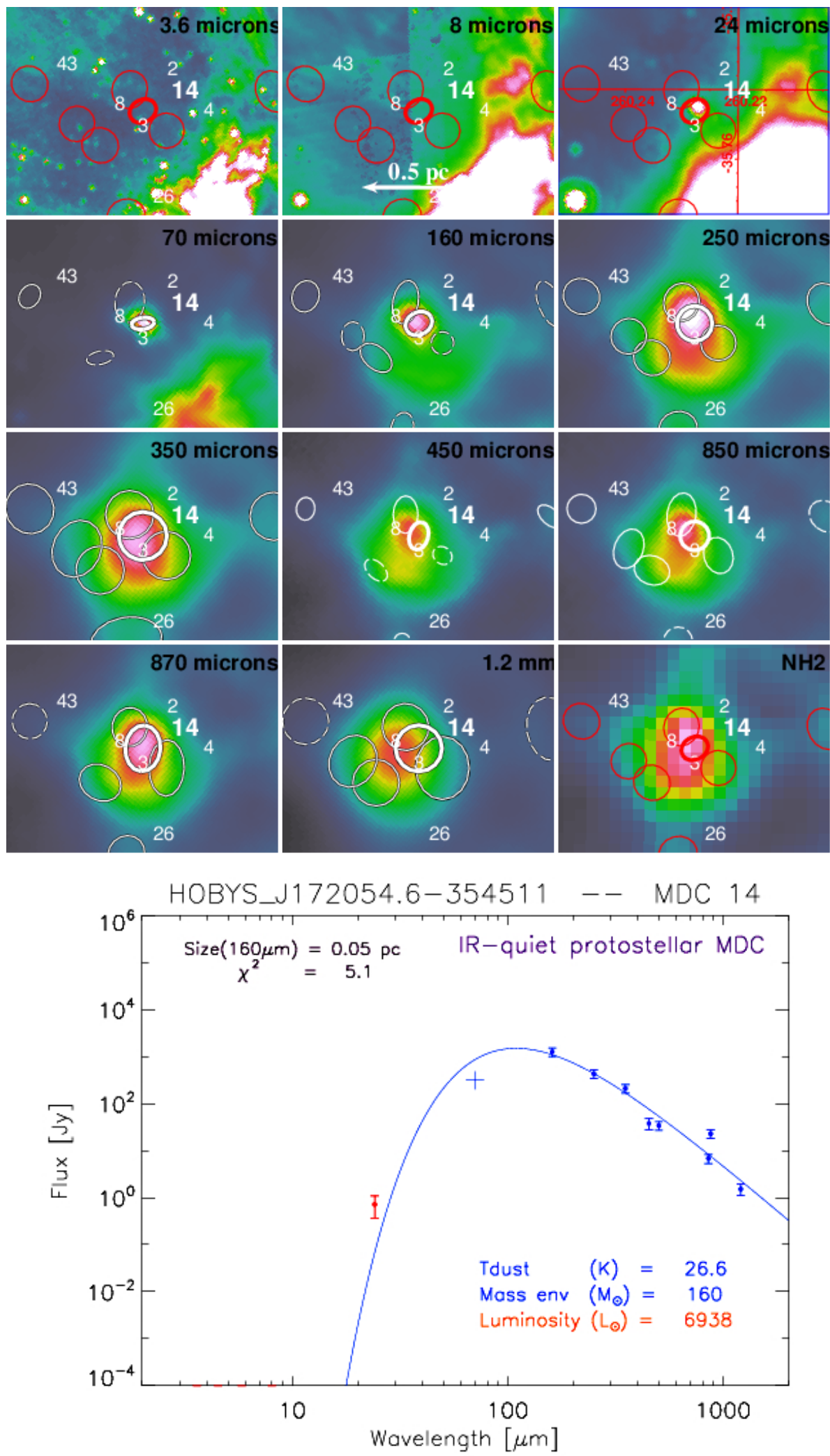

Fig. B.1. continued. 
A\&A 602, A77 (2017)
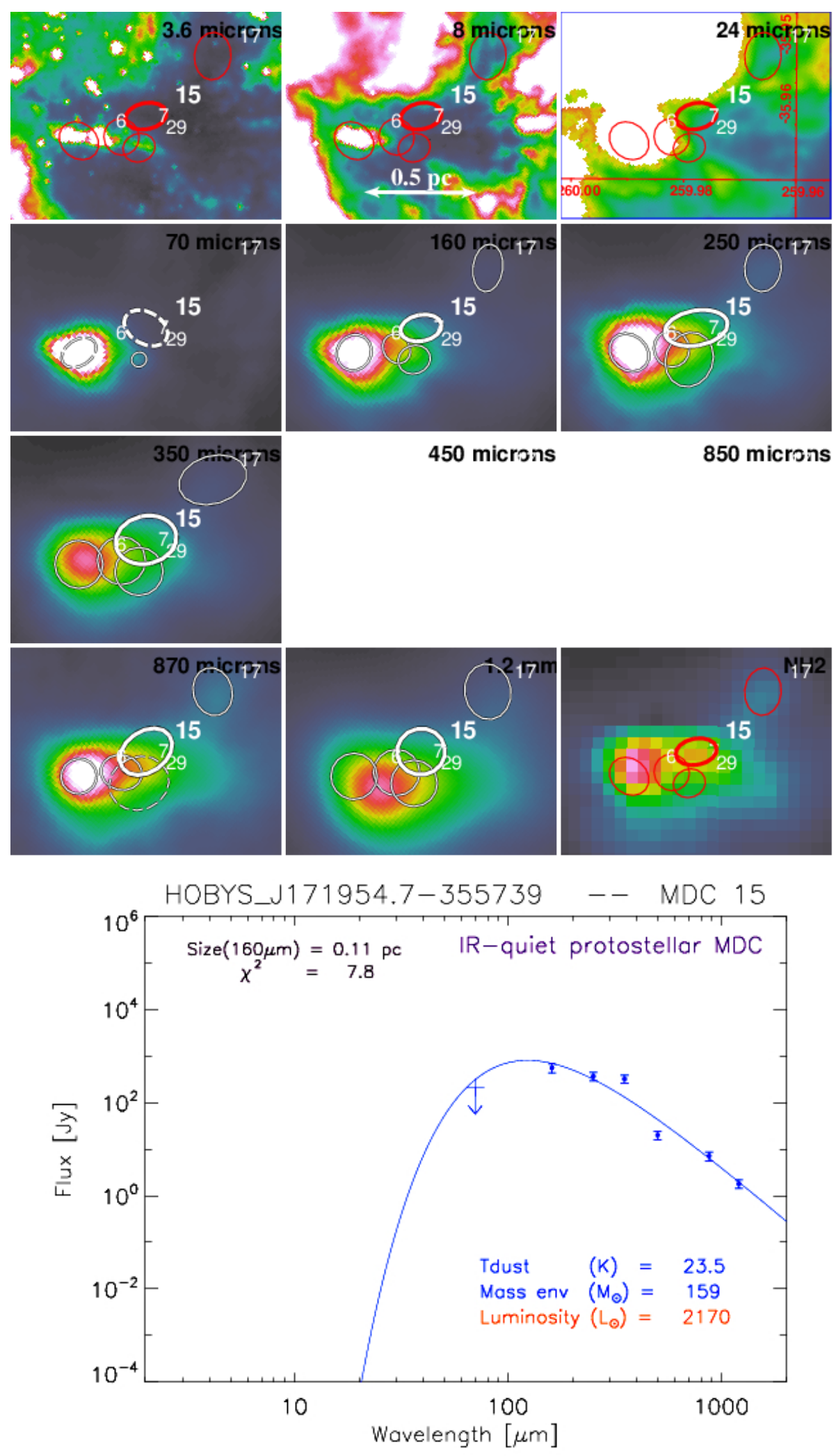

Fig. B.1. continued. 
J. Tigé et al.: The earliest phases of high-mass star formation in NGC 6334
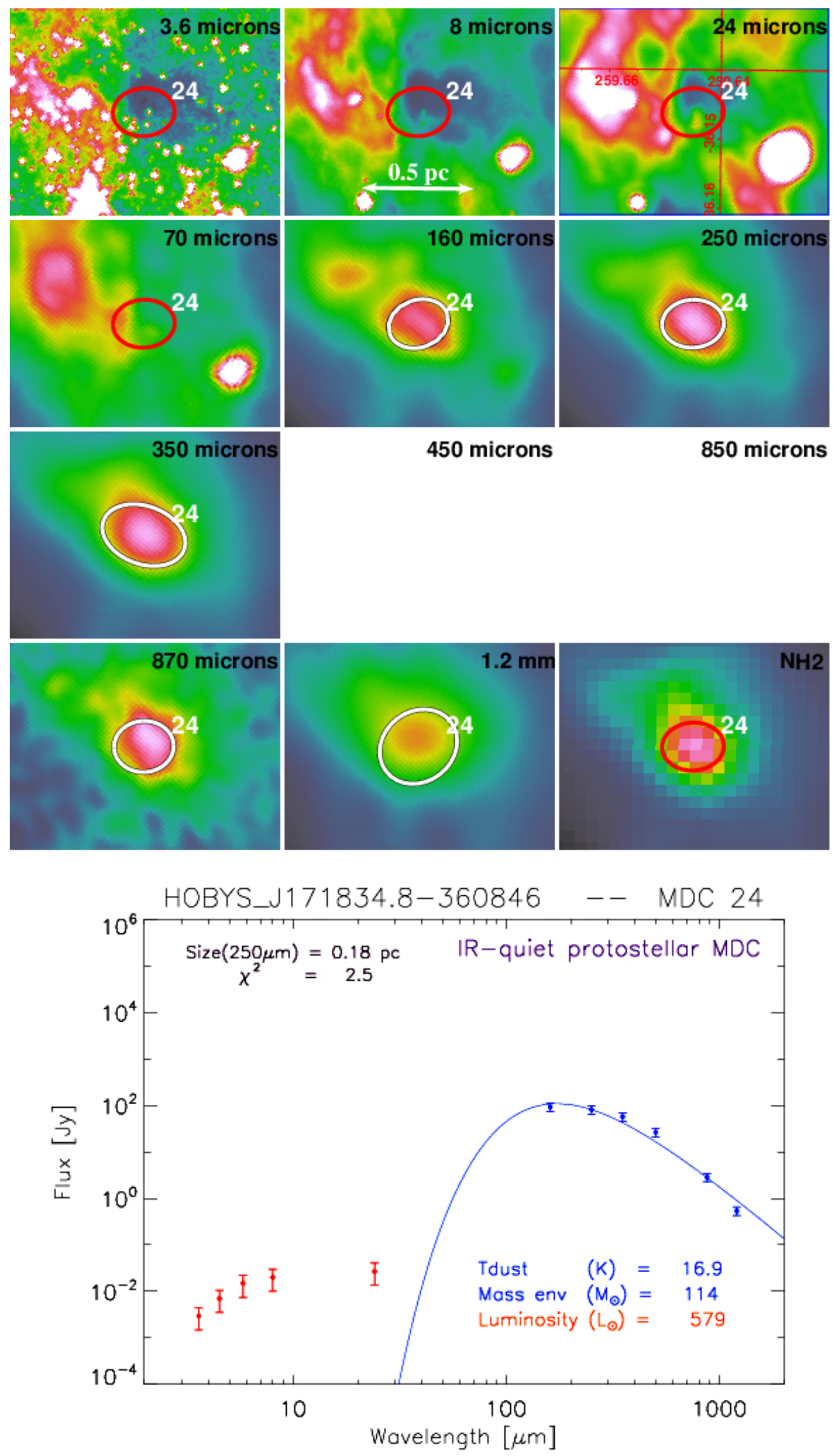

Fig. B.1. continued. 
A\&A 602, A77 (2017)
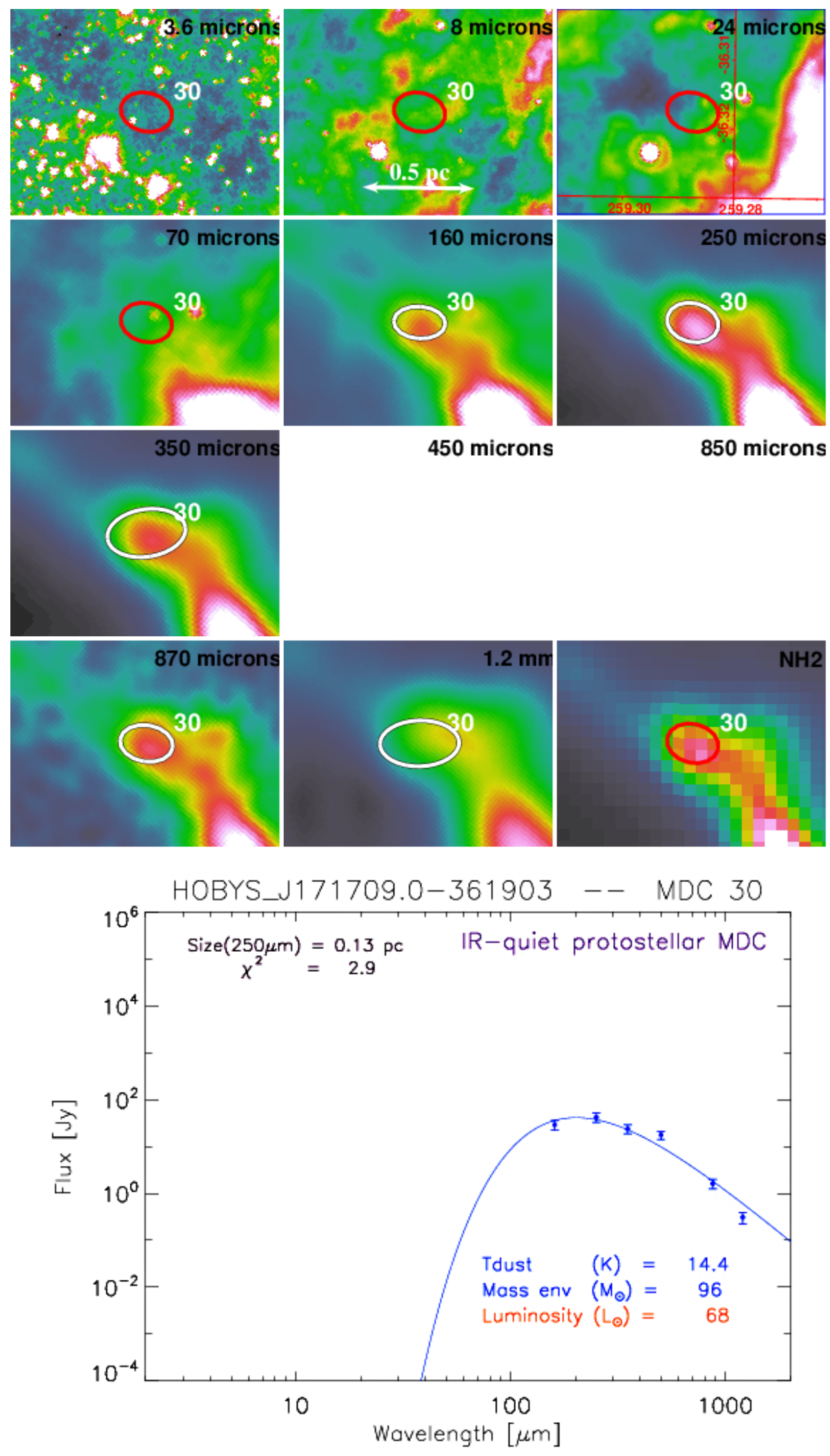

Fig. B.1. continued. 
J. Tigé et al.: The earliest phases of high-mass star formation in NGC 6334
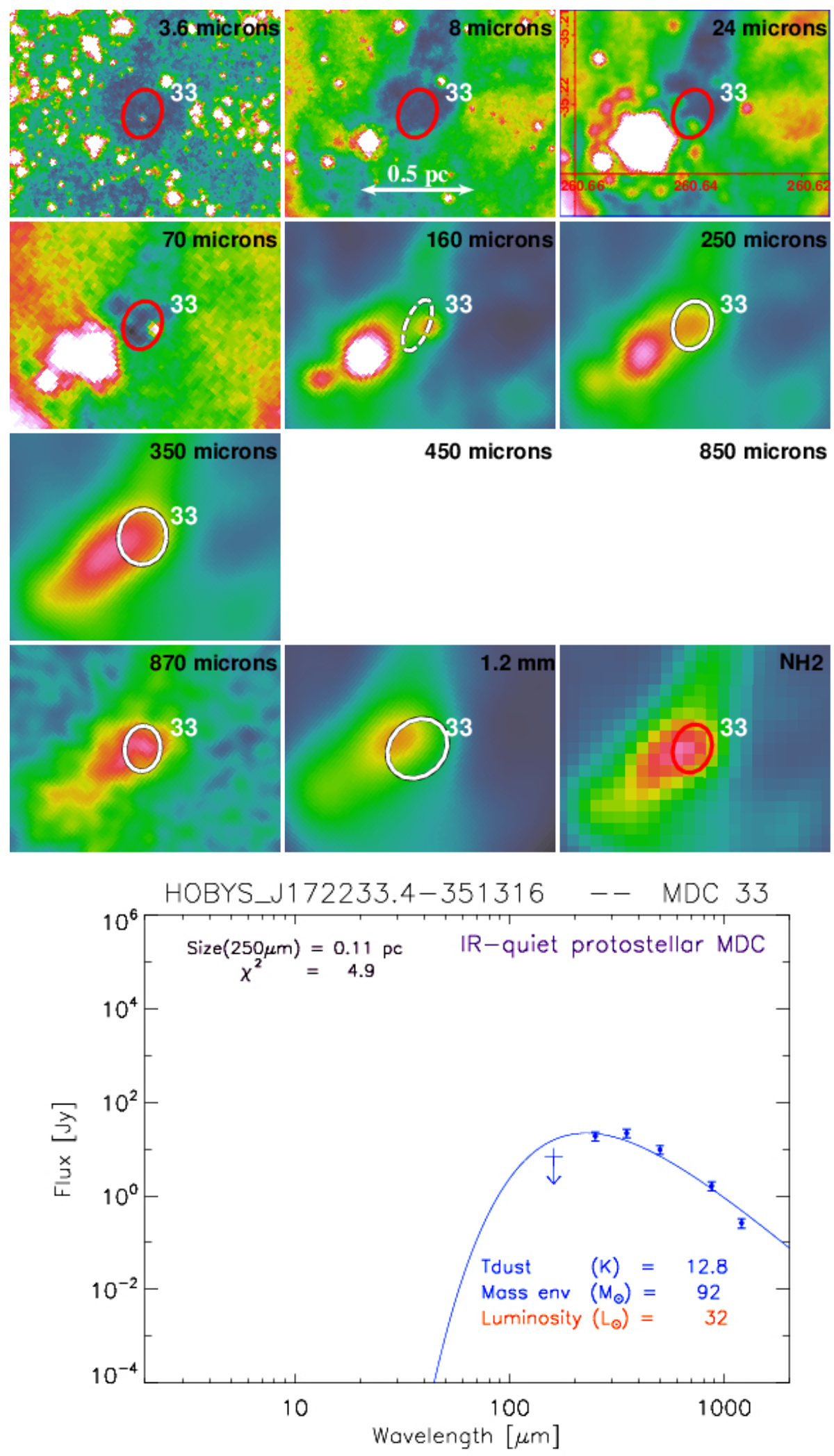

Fig. B.1. continued. 
A\&A 602, A77 (2017)
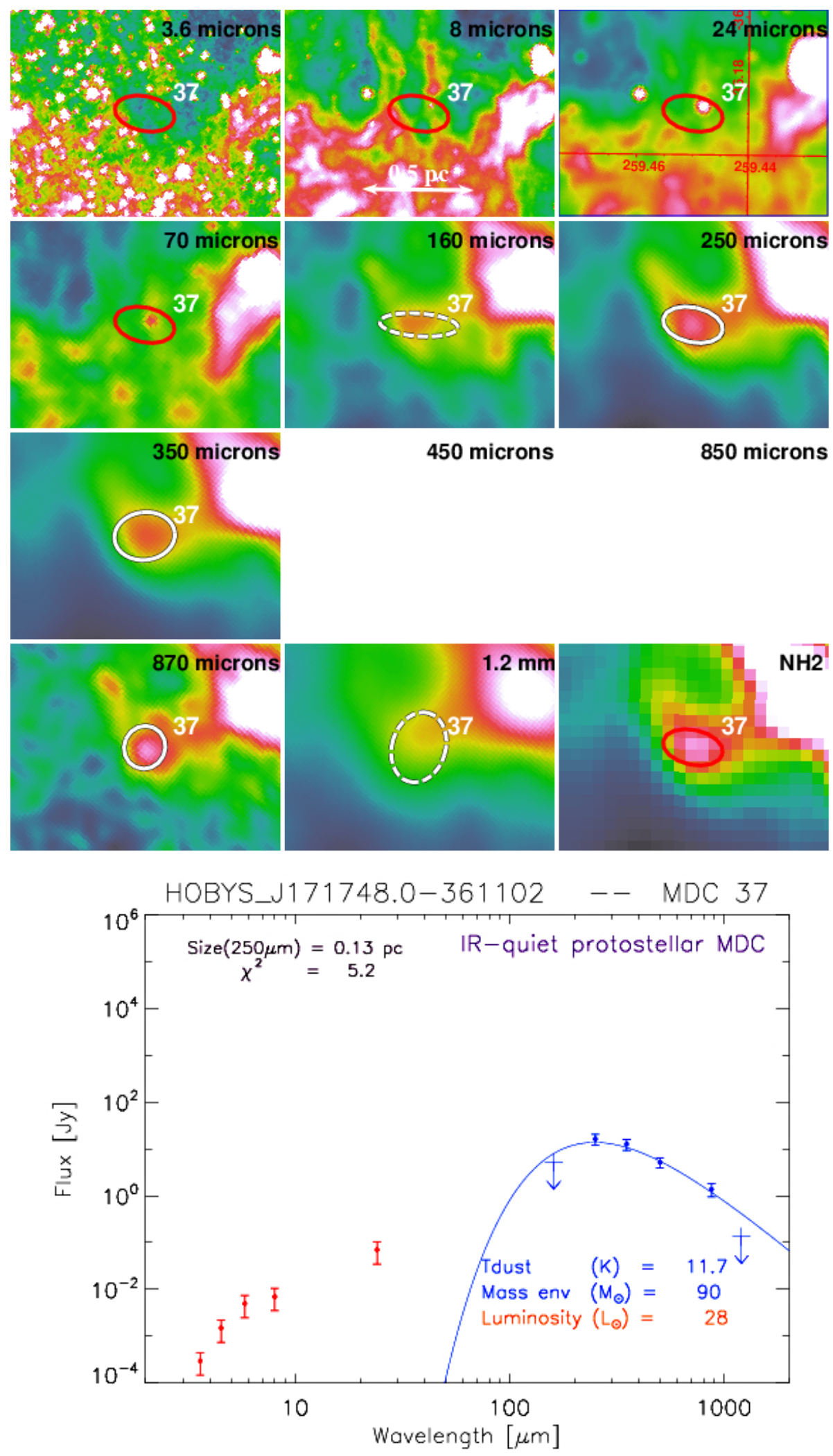

Fig. B.1. continued. 
J. Tigé et al.: The earliest phases of high-mass star formation in NGC 6334
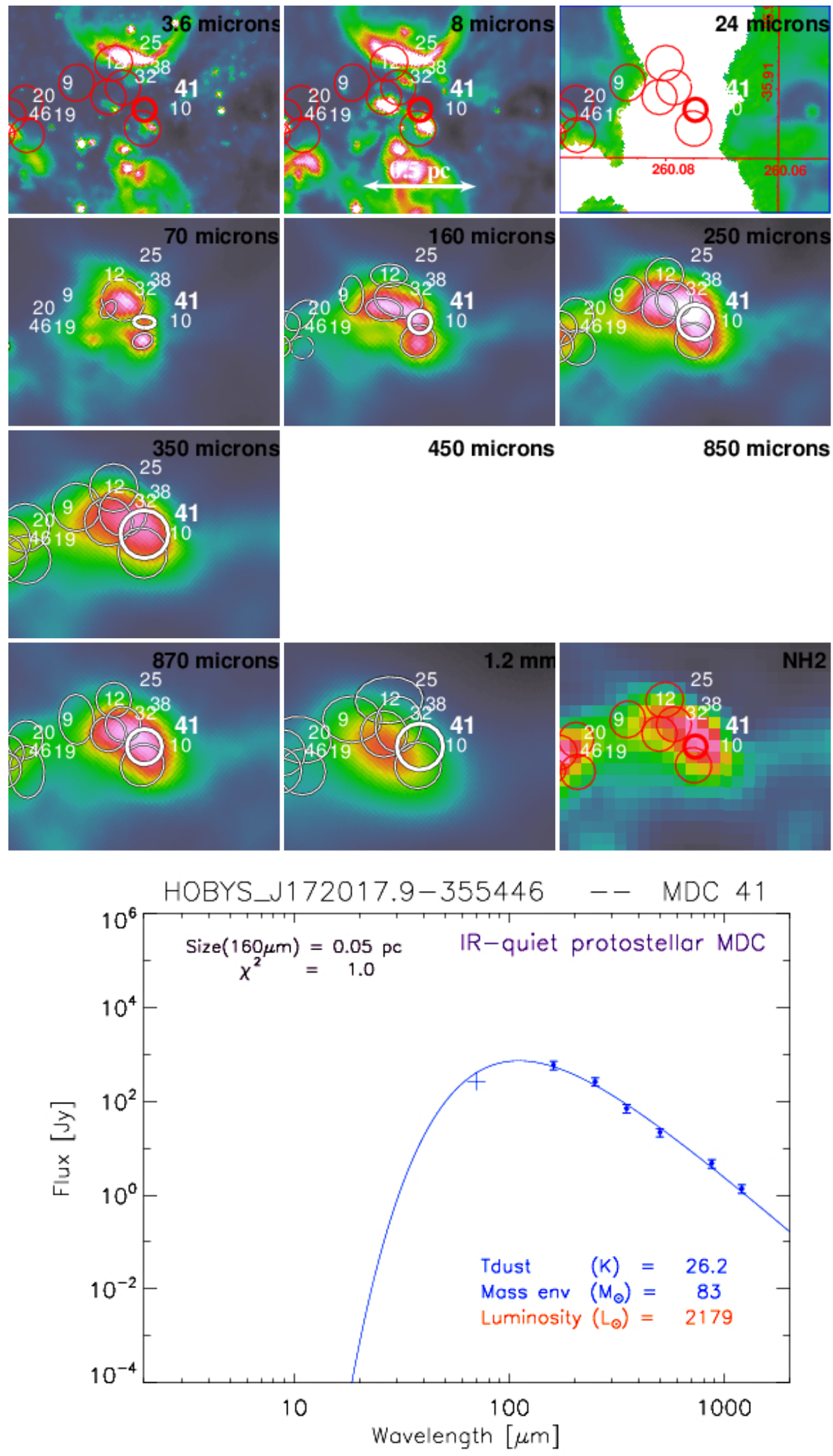

Fig. B.1. continued. 
A\&A 602, A77 (2017)
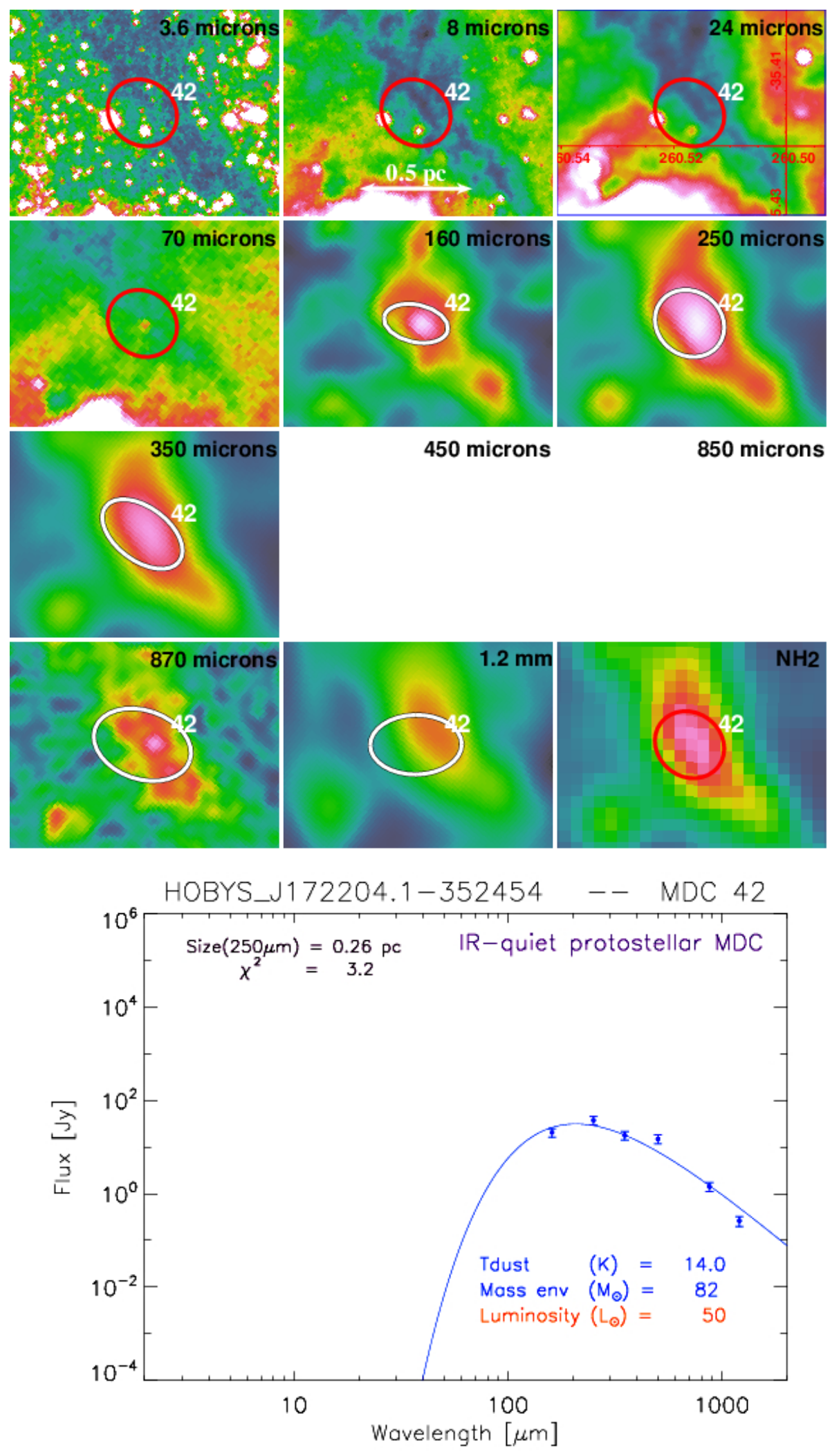

Fig. B.1. continued. 


\section{B.3. Starless MDCs}
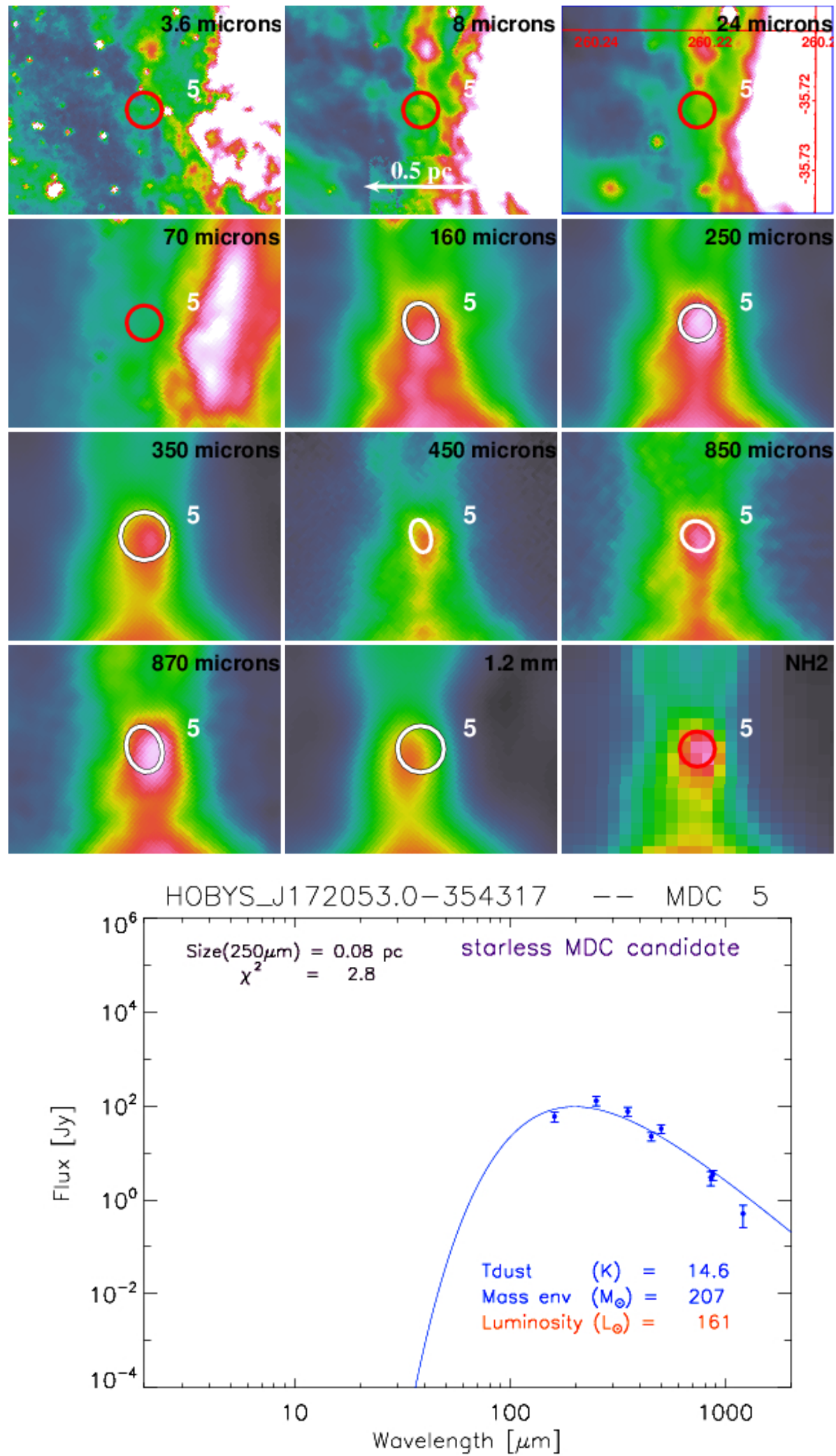

Fig. B.1. continued. 
A\&A 602, A77 (2017)
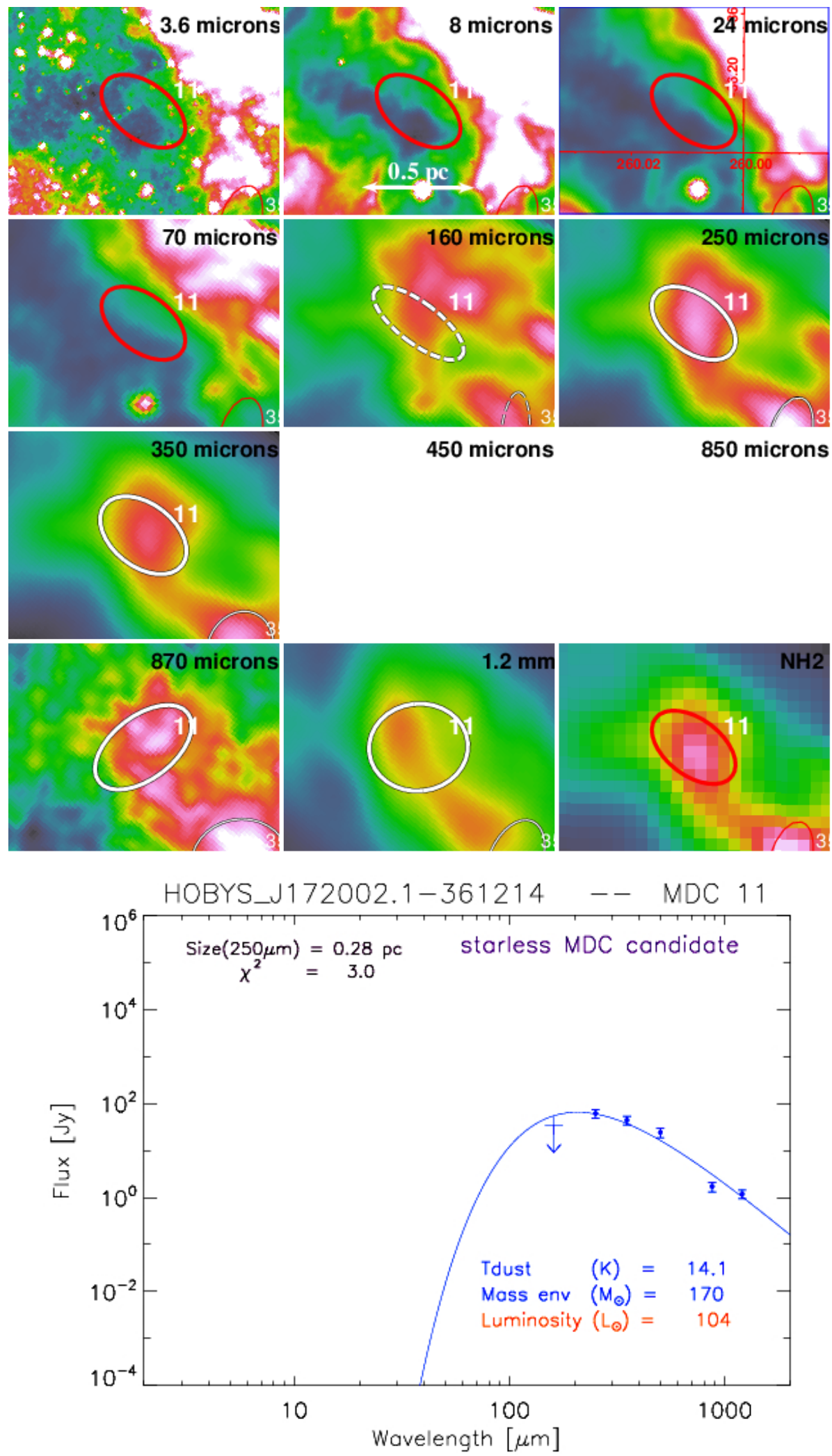

Fig. B.1. continued. 
J. Tigé et al.: The earliest phases of high-mass star formation in NGC 6334
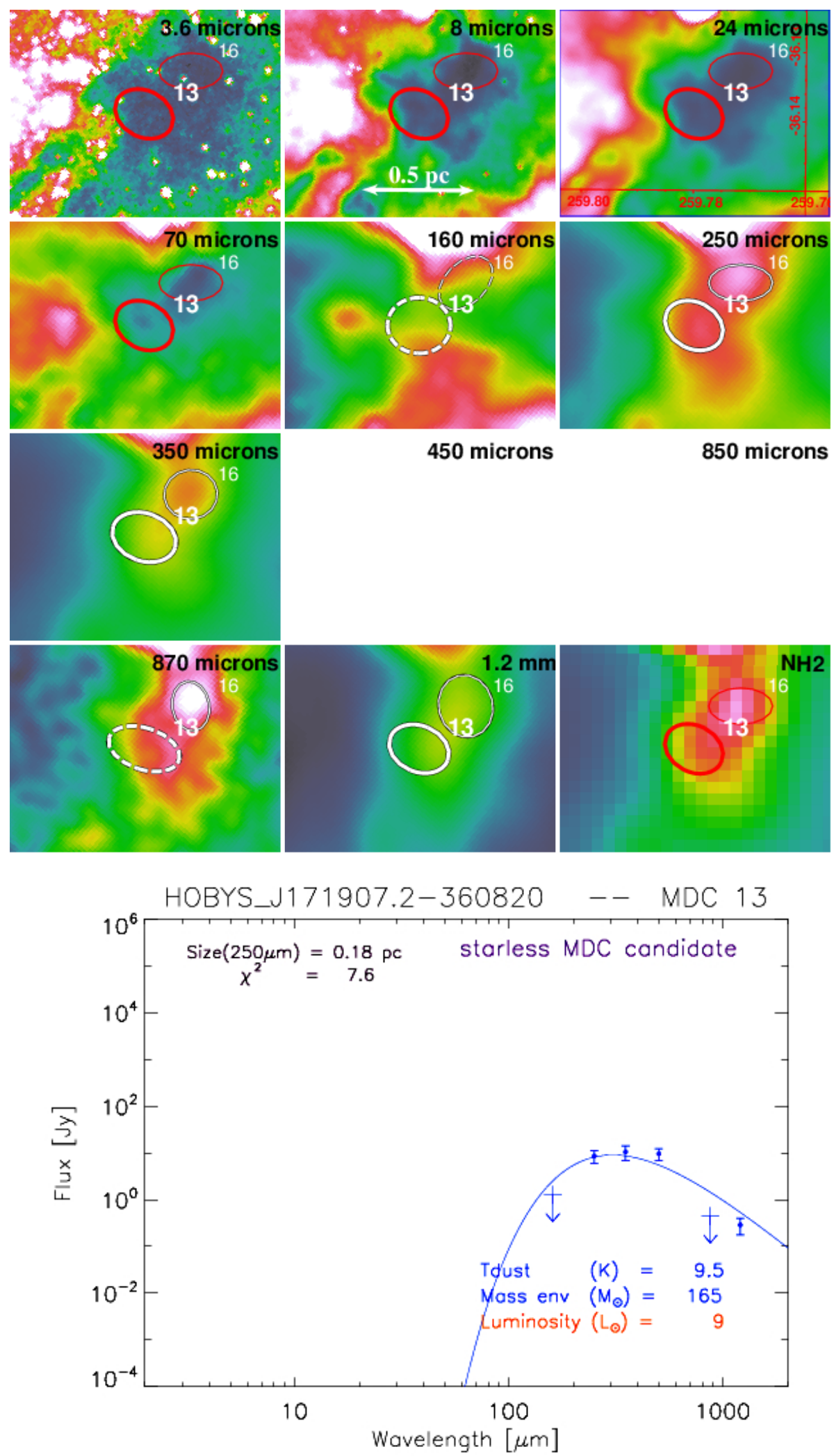

Fig. B.1. continued. 
A\&A 602, A77 (2017)
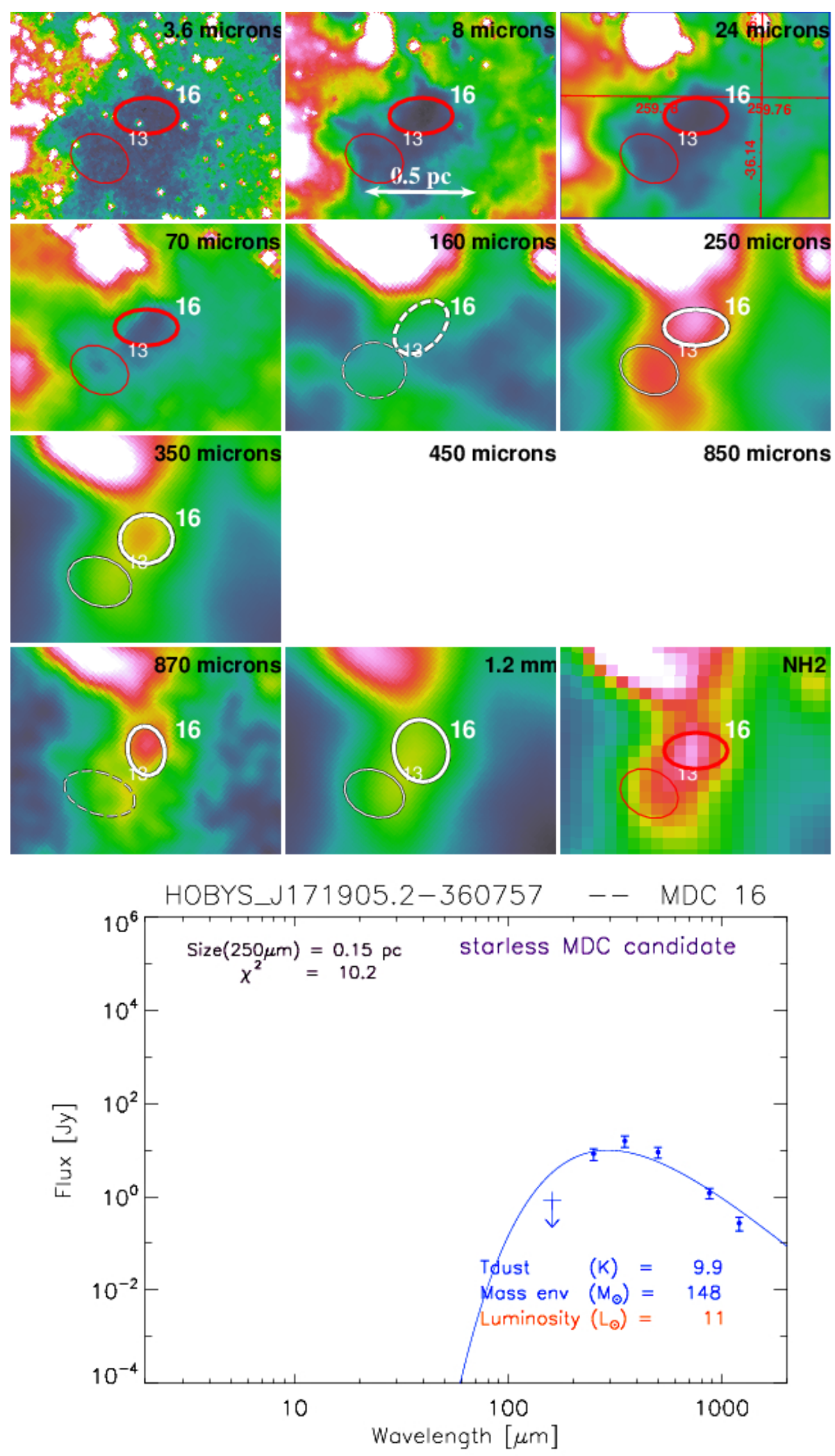

Fig. B.1. continued. 
J. Tigé et al.: The earliest phases of high-mass star formation in NGC 6334
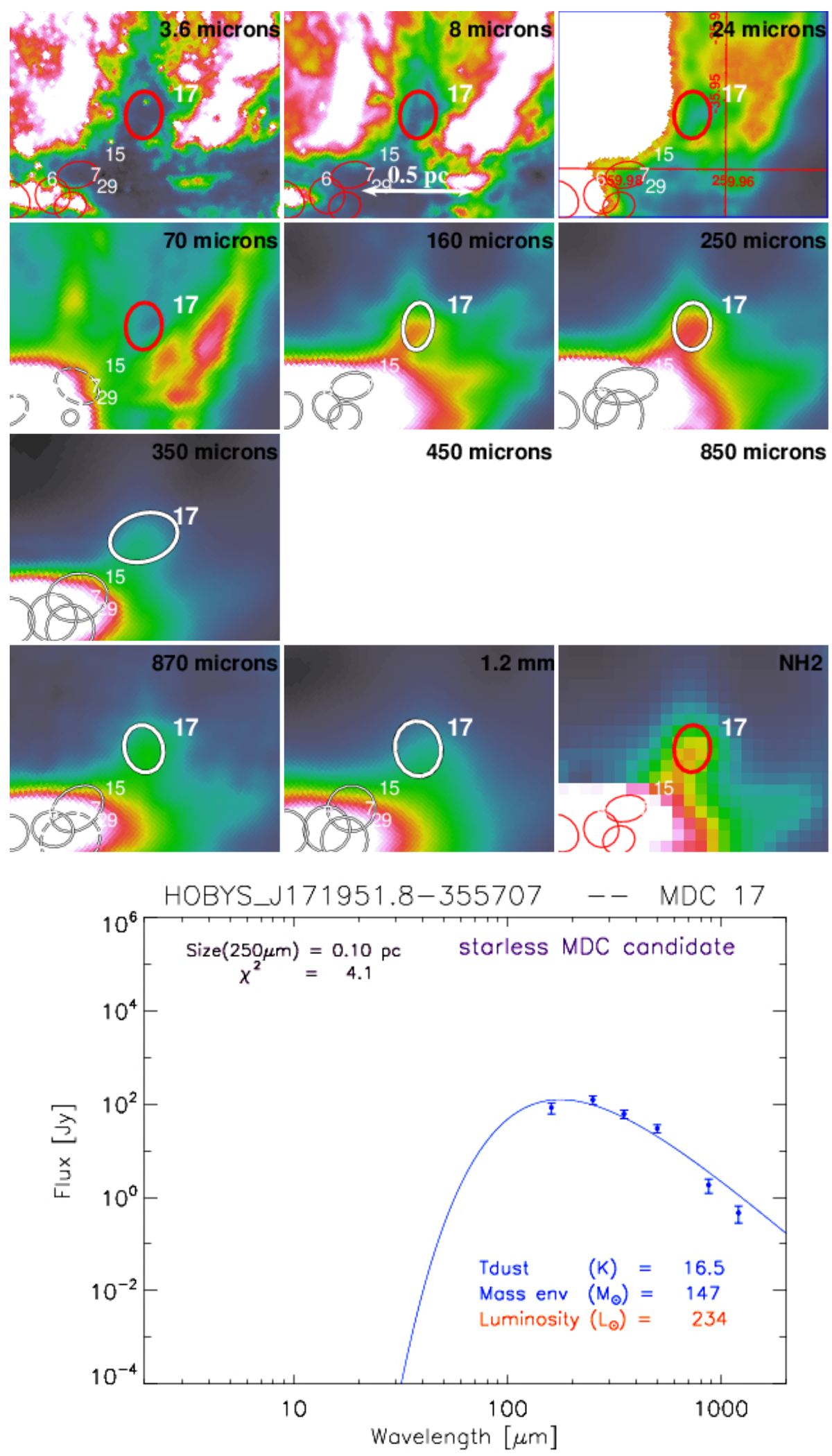

Fig. B.1. continued. 
A\&A 602, A77 (2017)
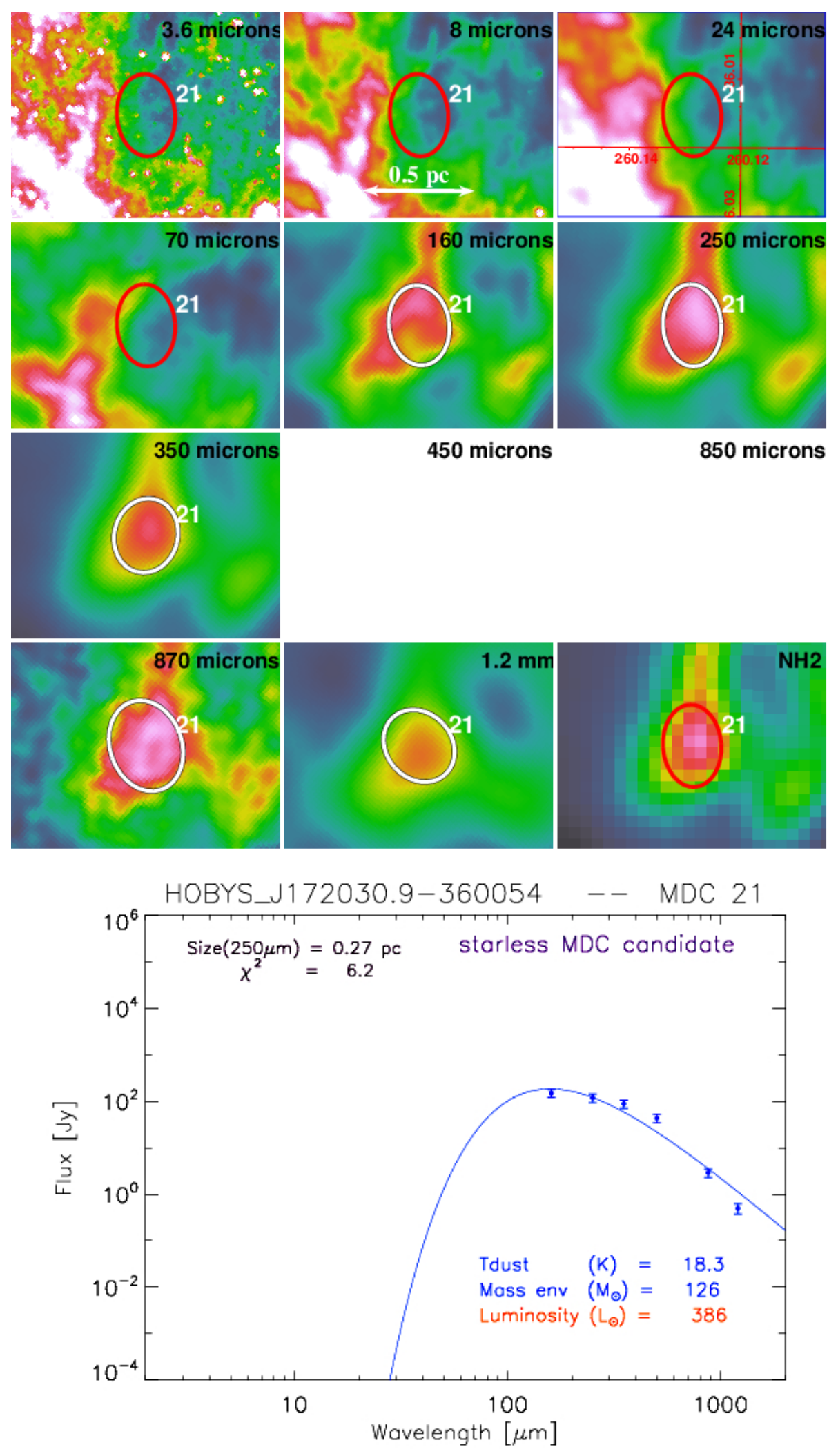

Fig. B.1. continued. 
J. Tigé et al.: The earliest phases of high-mass star formation in NGC 6334

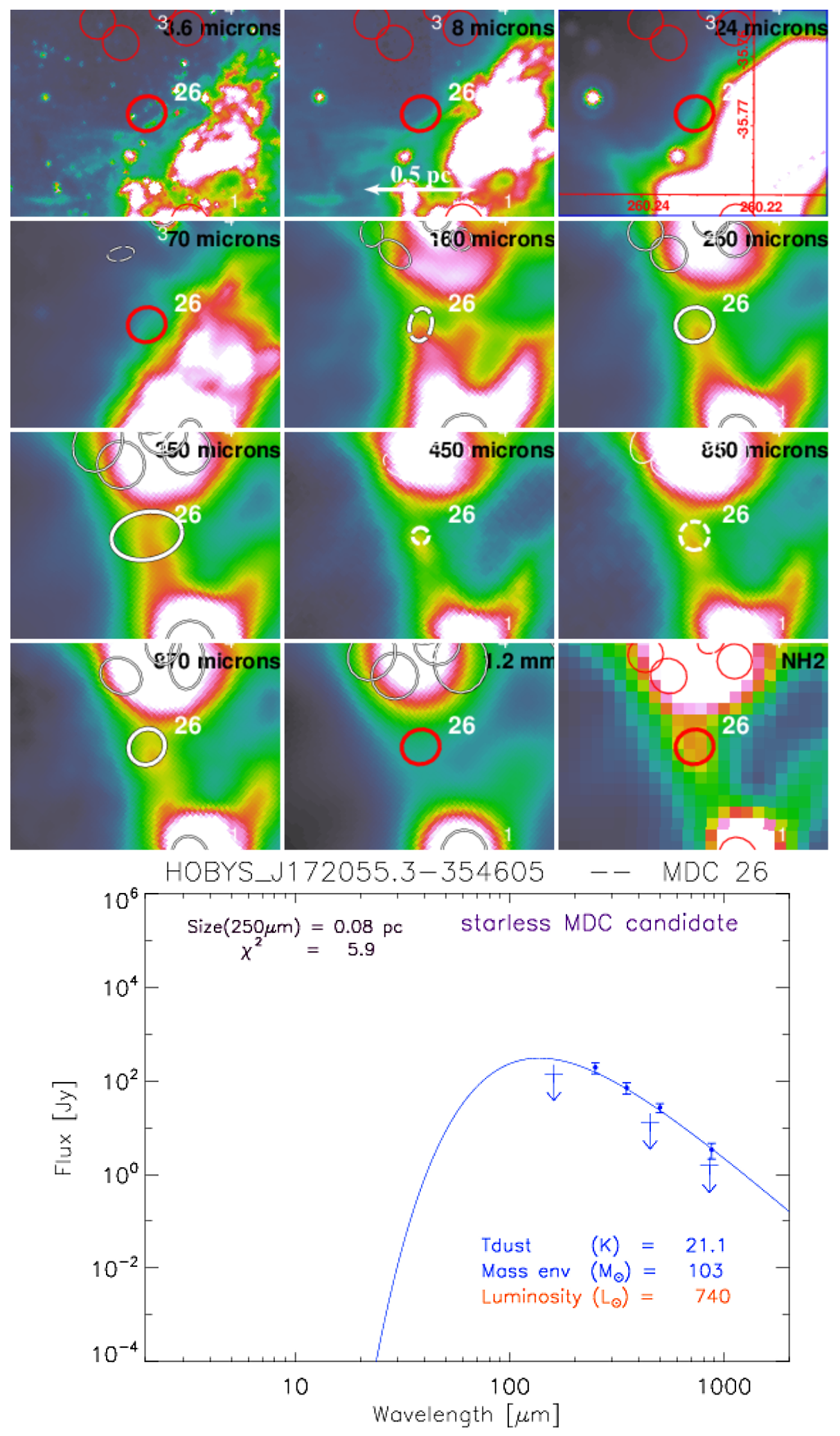

Fig. B.1. continued. 
A\&A 602, A77 (2017)

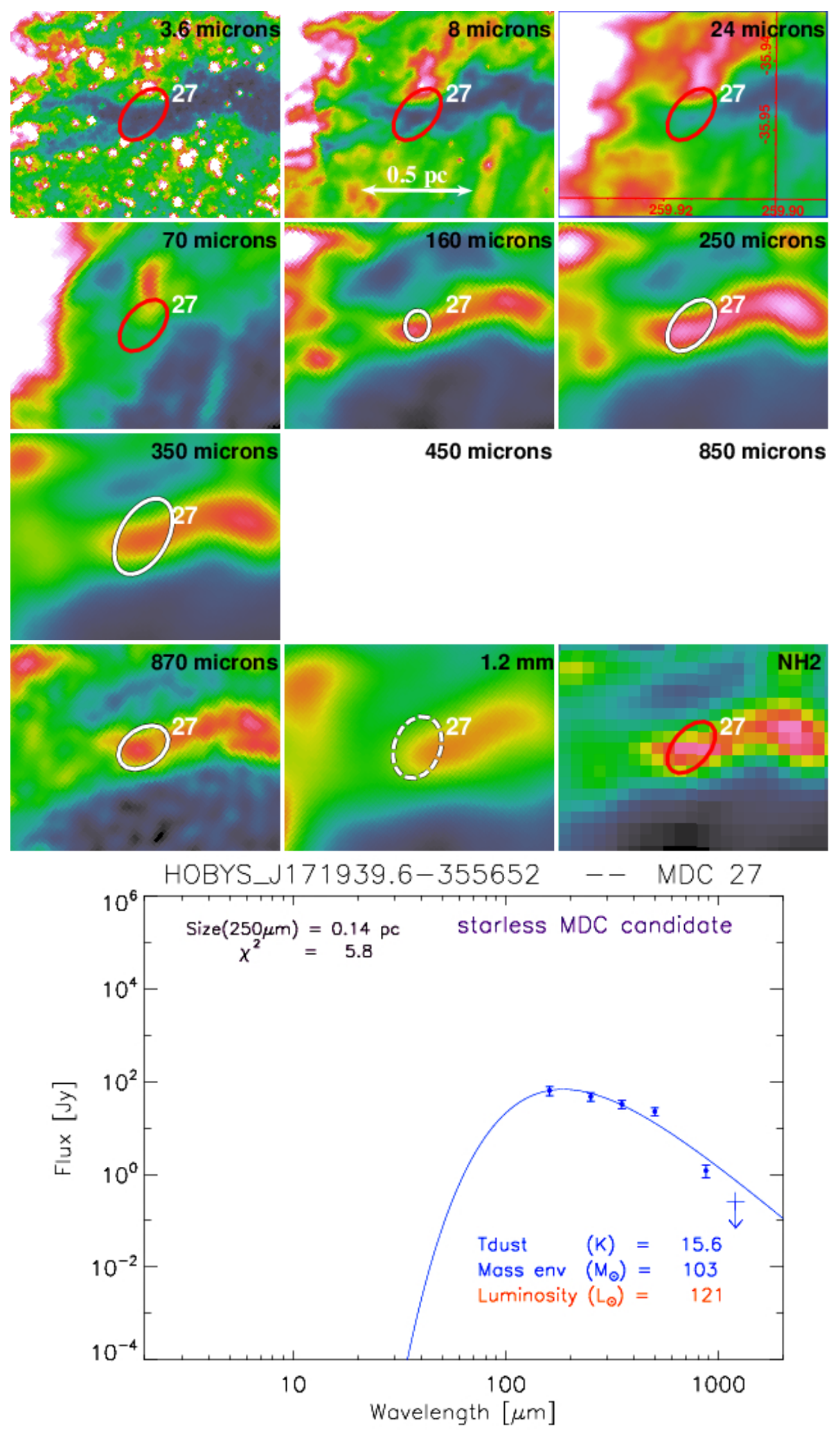

Fig. B.1. continued. 
J. Tigé et al.: The earliest phases of high-mass star formation in NGC 6334

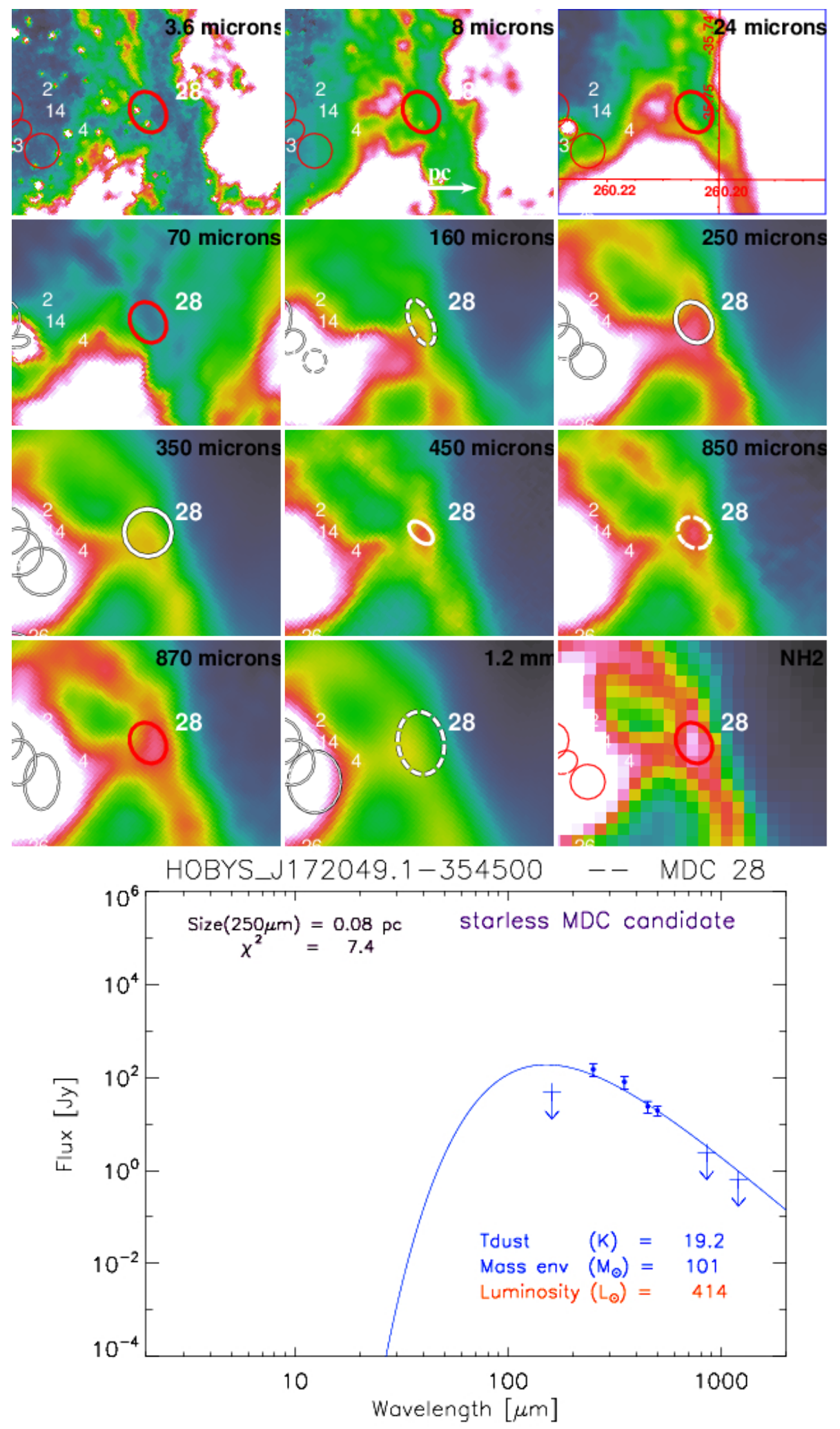

Fig. B.1. continued. 
A\&A 602, A77 (2017)

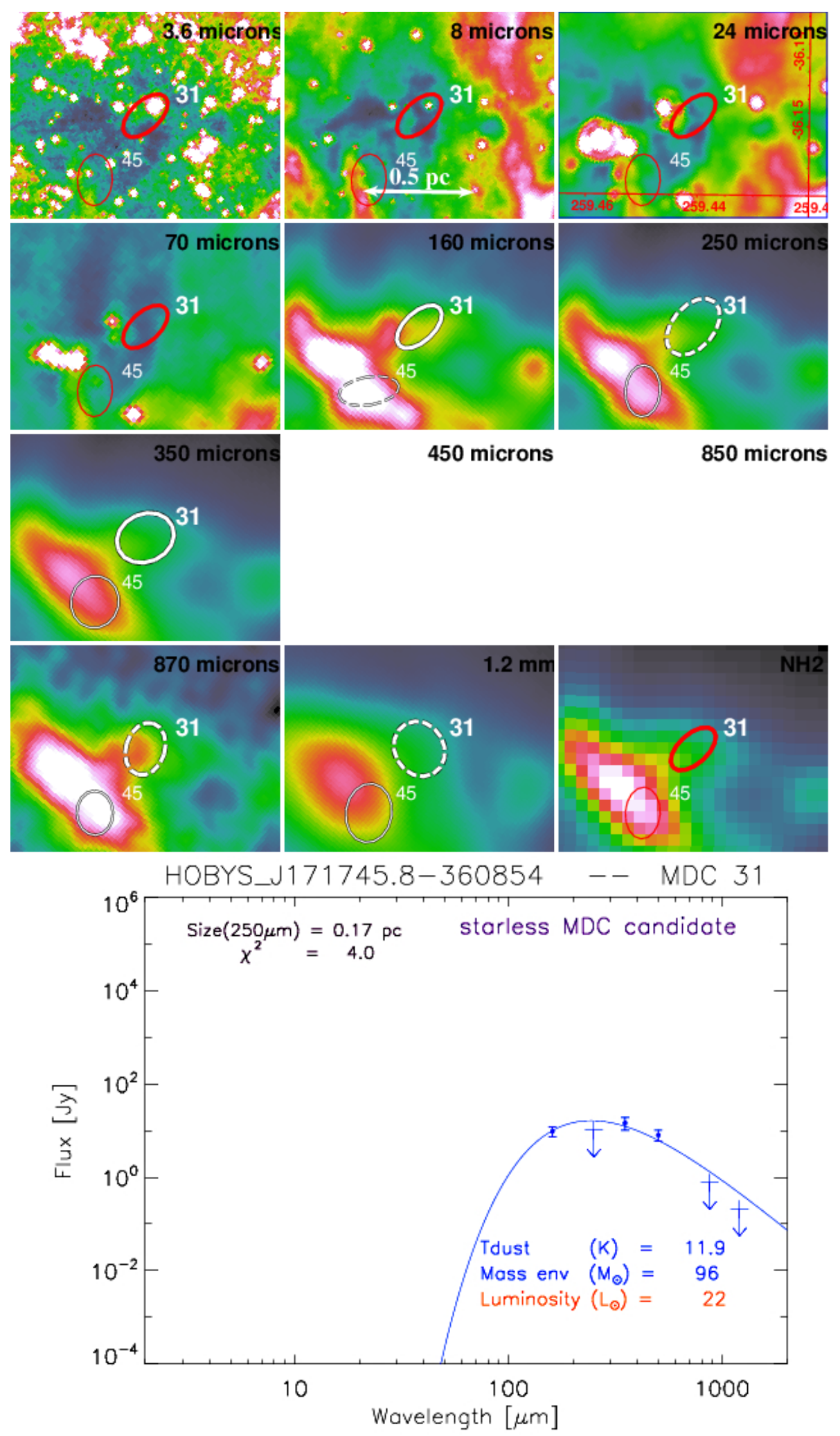

Fig. B.1. continued. 
J. Tigé et al.: The earliest phases of high-mass star formation in NGC 6334

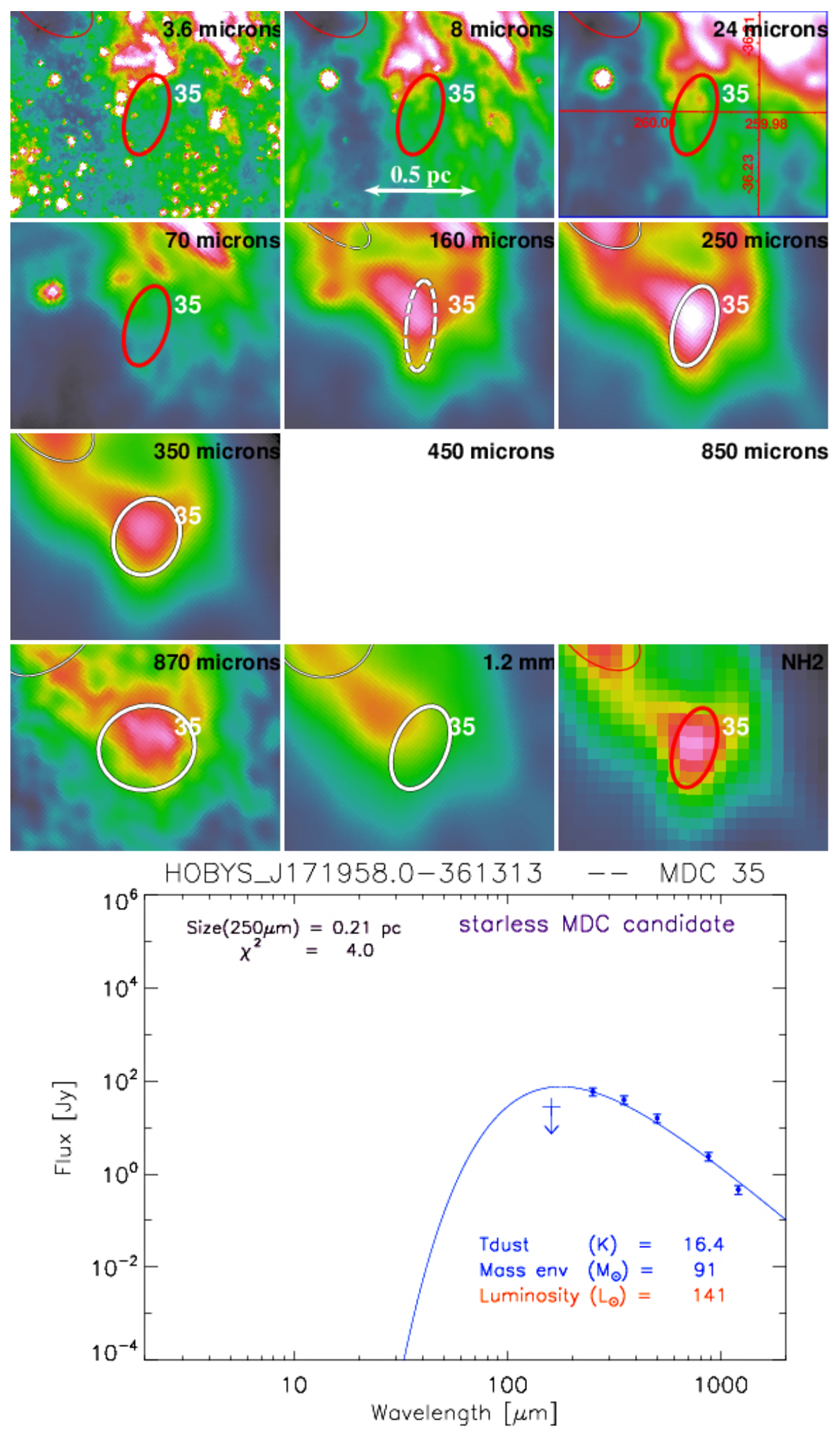

Fig. B.1. continued. 
A\&A 602, A77 (2017)

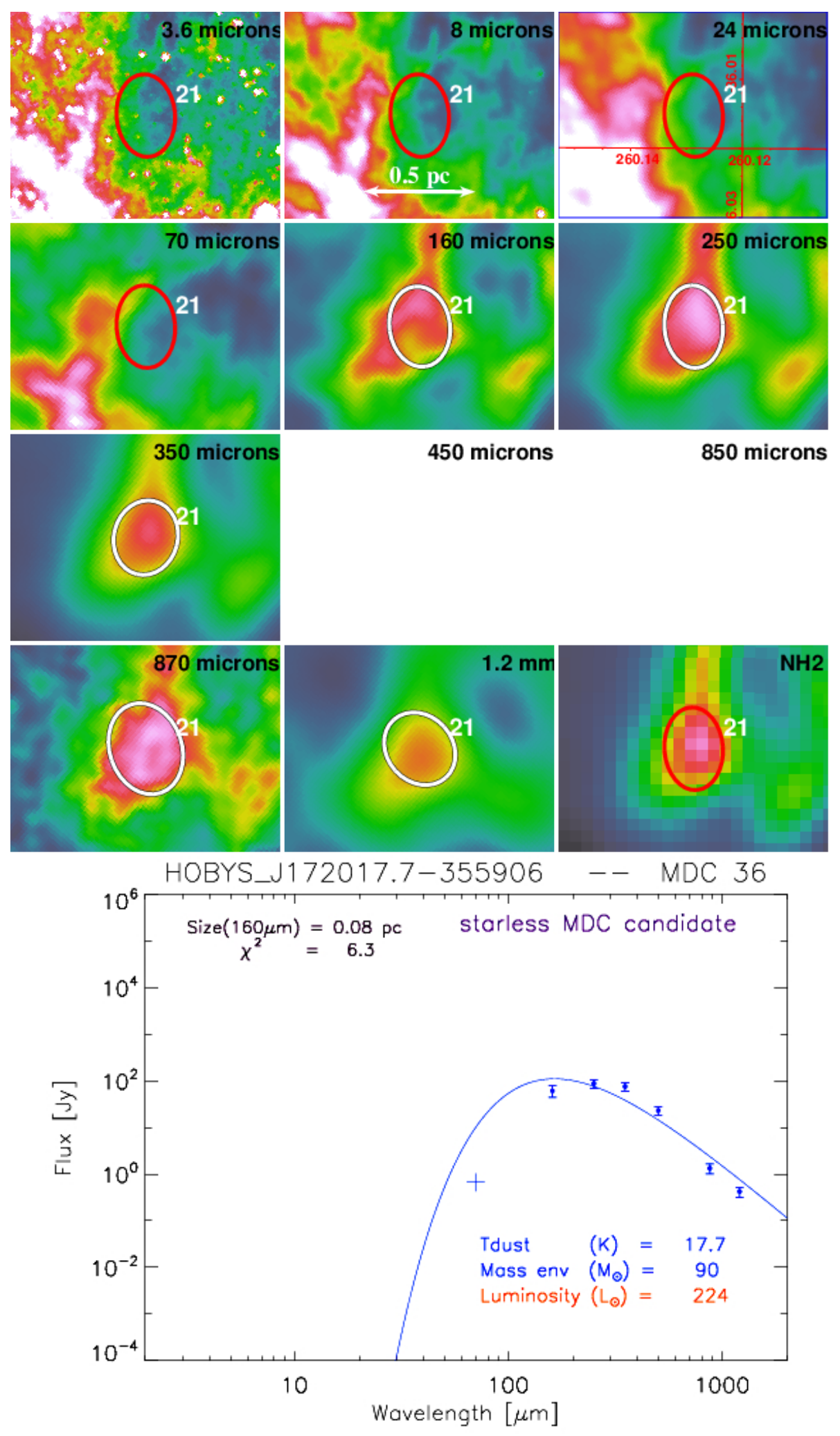

Fig. B.1. continued. 
J. Tigé et al.: The earliest phases of high-mass star formation in NGC 6334

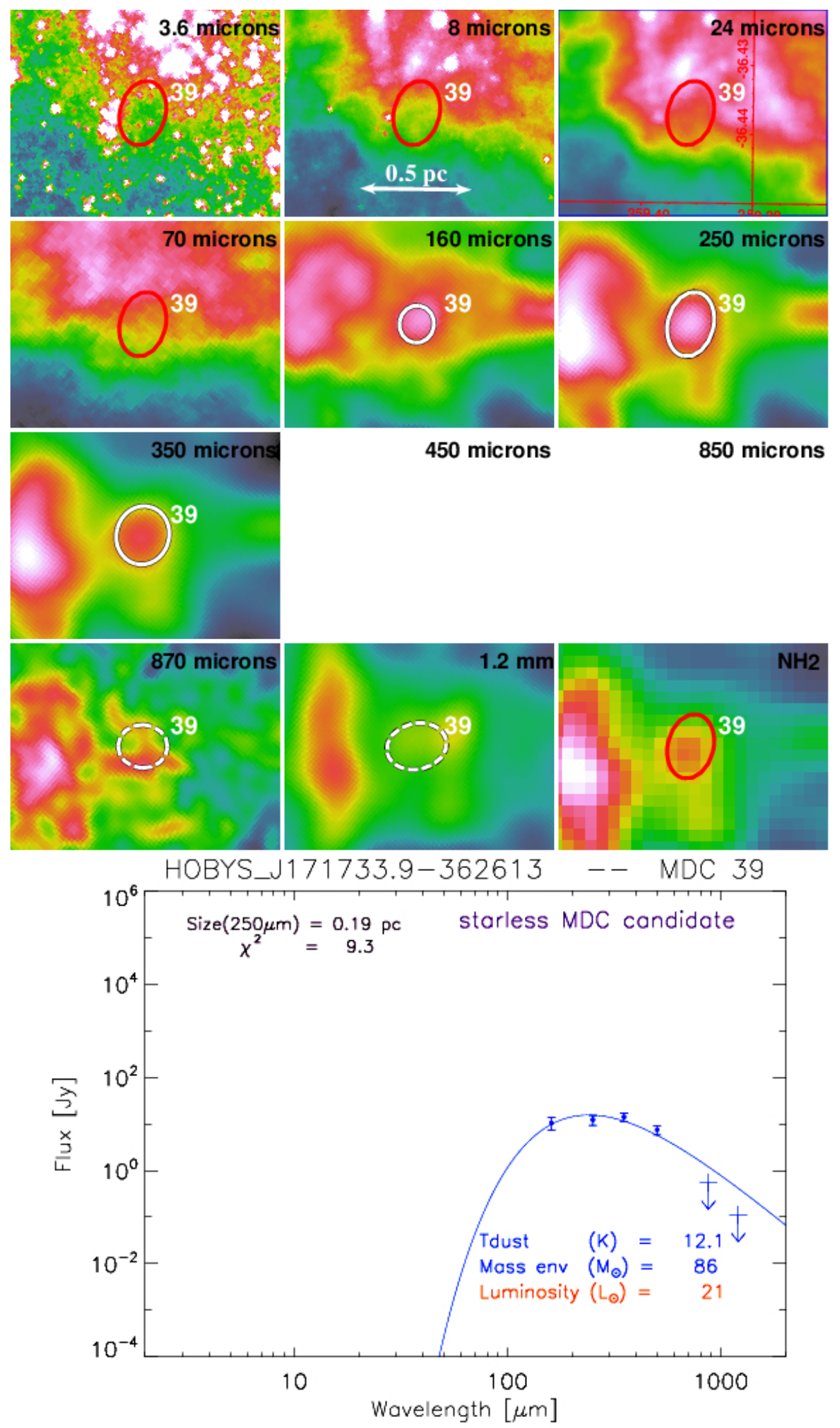

Fig. B.1. continued. 
A\&A 602, A77 (2017)

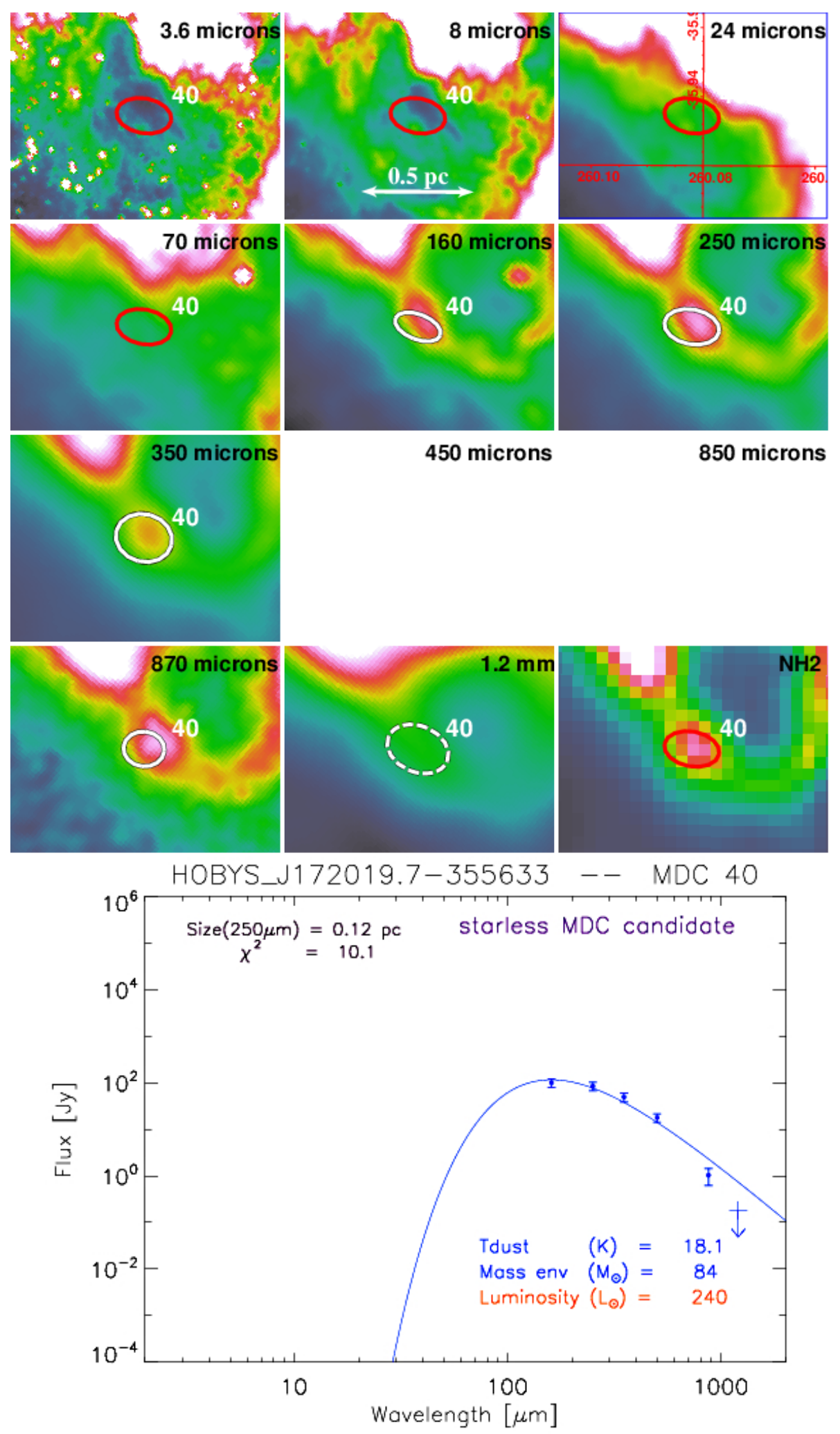

Fig. B.1. continued. 
J. Tigé et al.: The earliest phases of high-mass star formation in NGC 6334

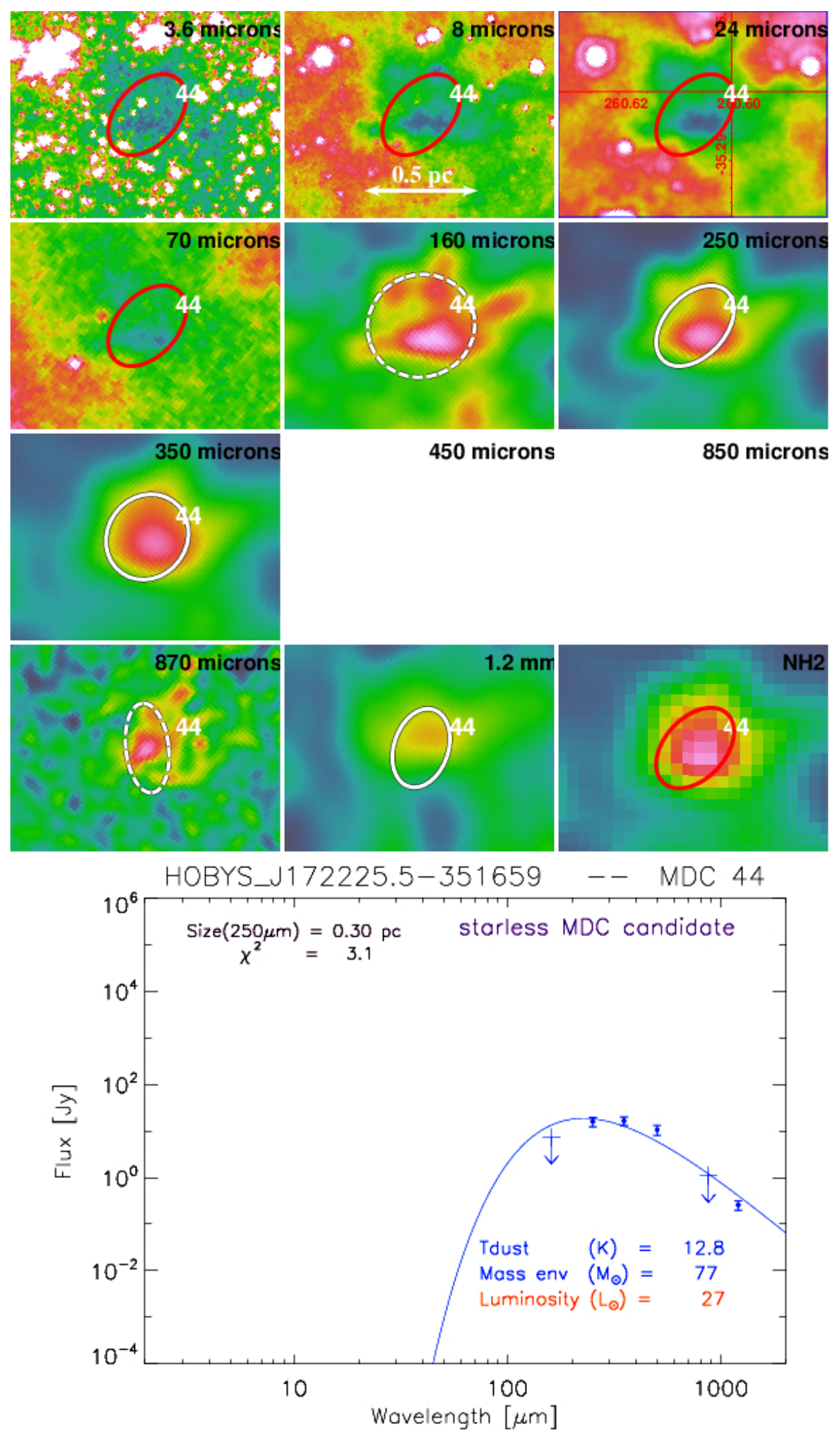

Fig. B.1. continued. 
A\&A 602, A77 (2017)

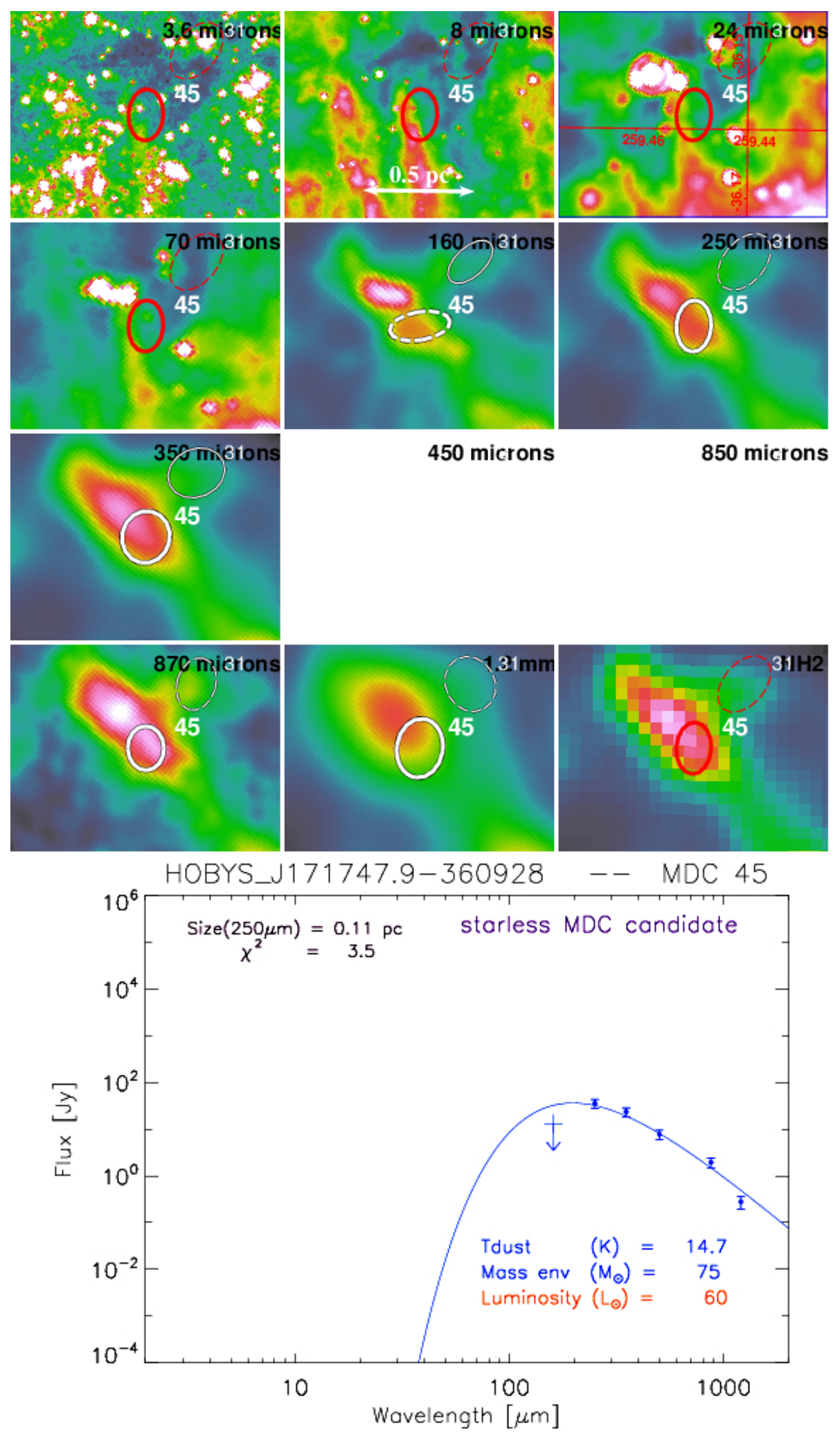

Fig. B.1. continued. 
J. Tigé et al.: The earliest phases of high-mass star formation in NGC 6334

\section{B.4. Undefined cloud structures}
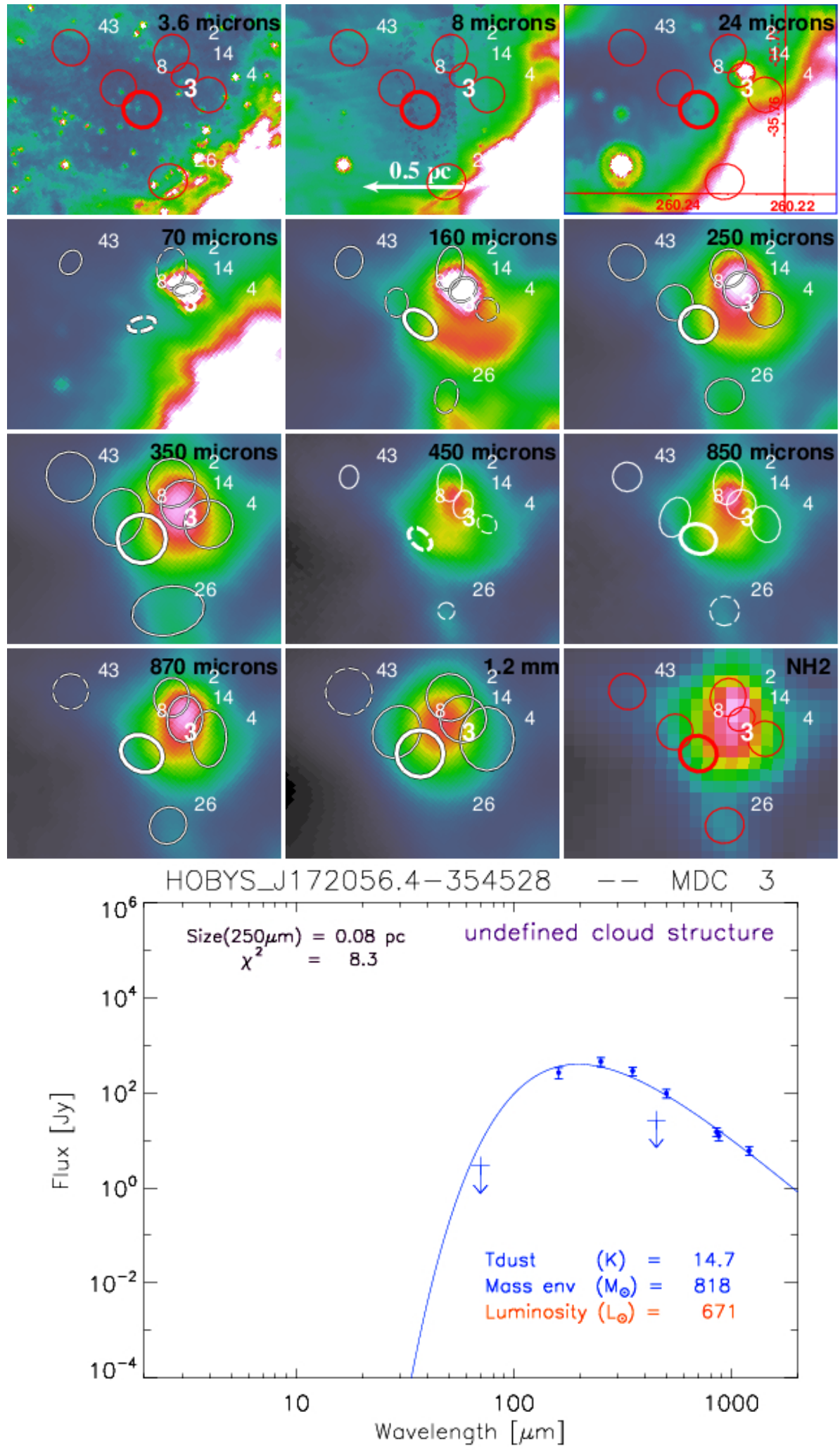

Fig. B.1. continued. 
A\&A 602, A77 (2017)

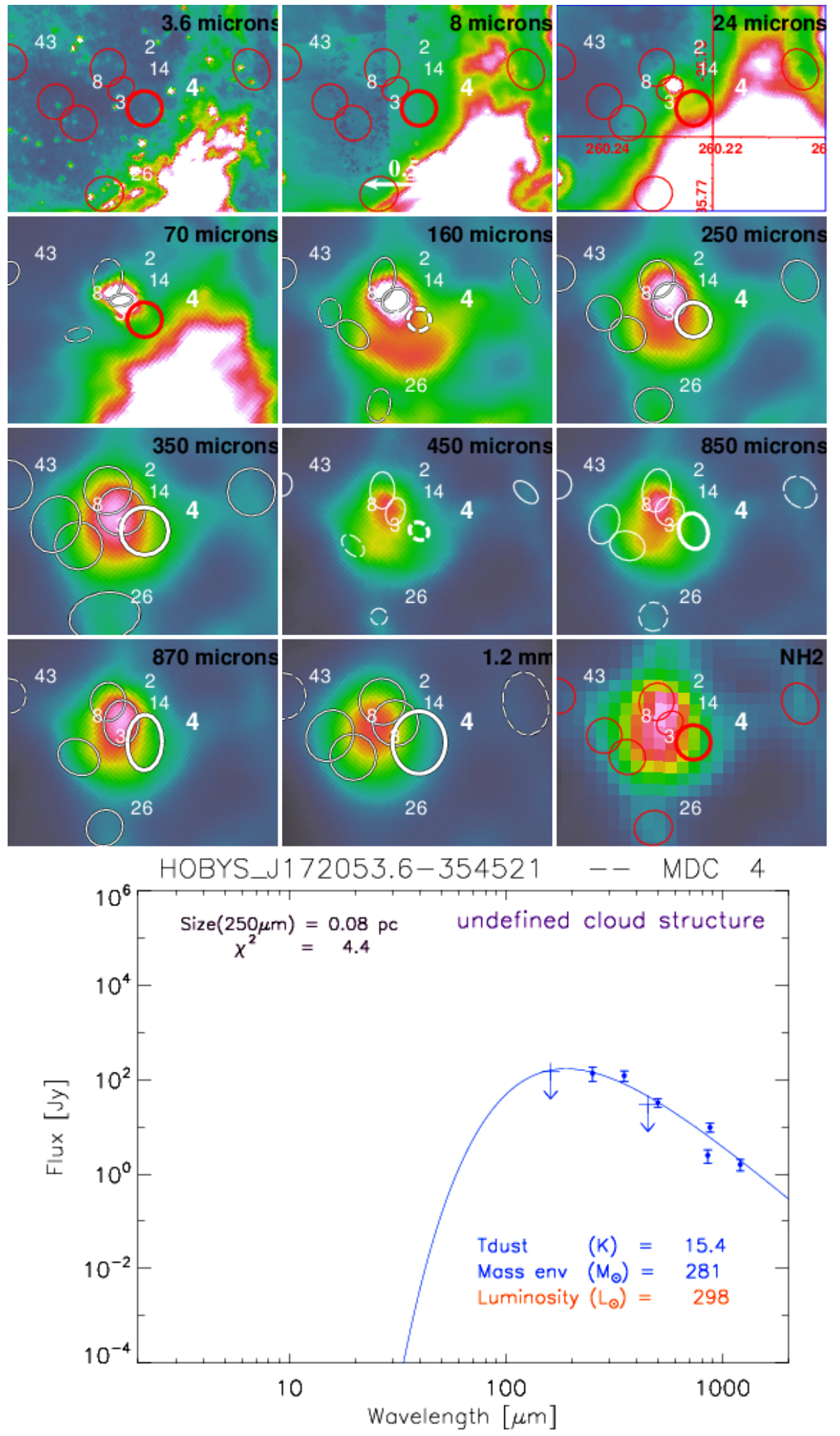

Fig. B.1. continued. 
J. Tigé et al.: The earliest phases of high-mass star formation in NGC 6334

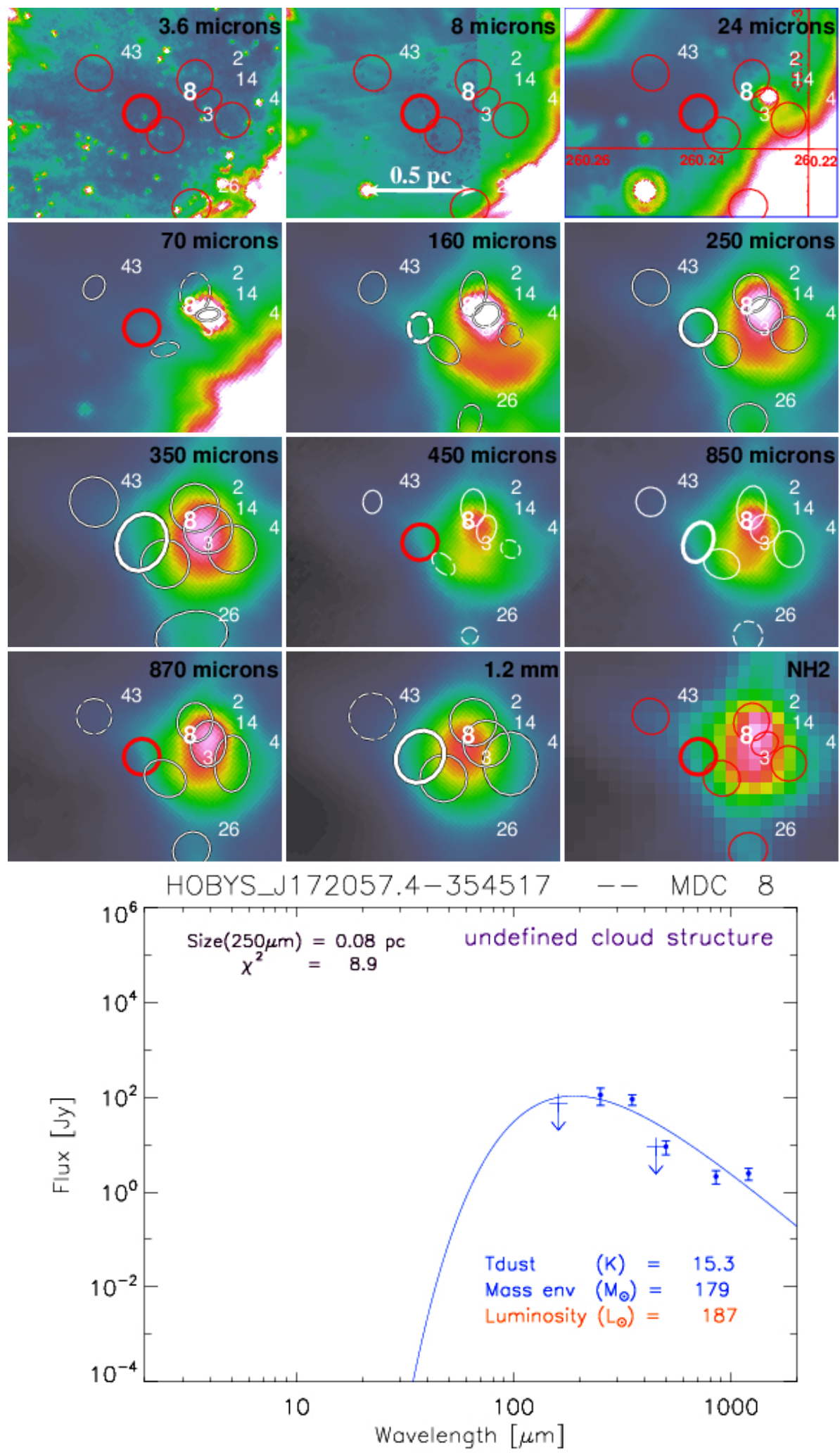

Fig. B.1. continued. 
A\&A 602, A77 (2017)

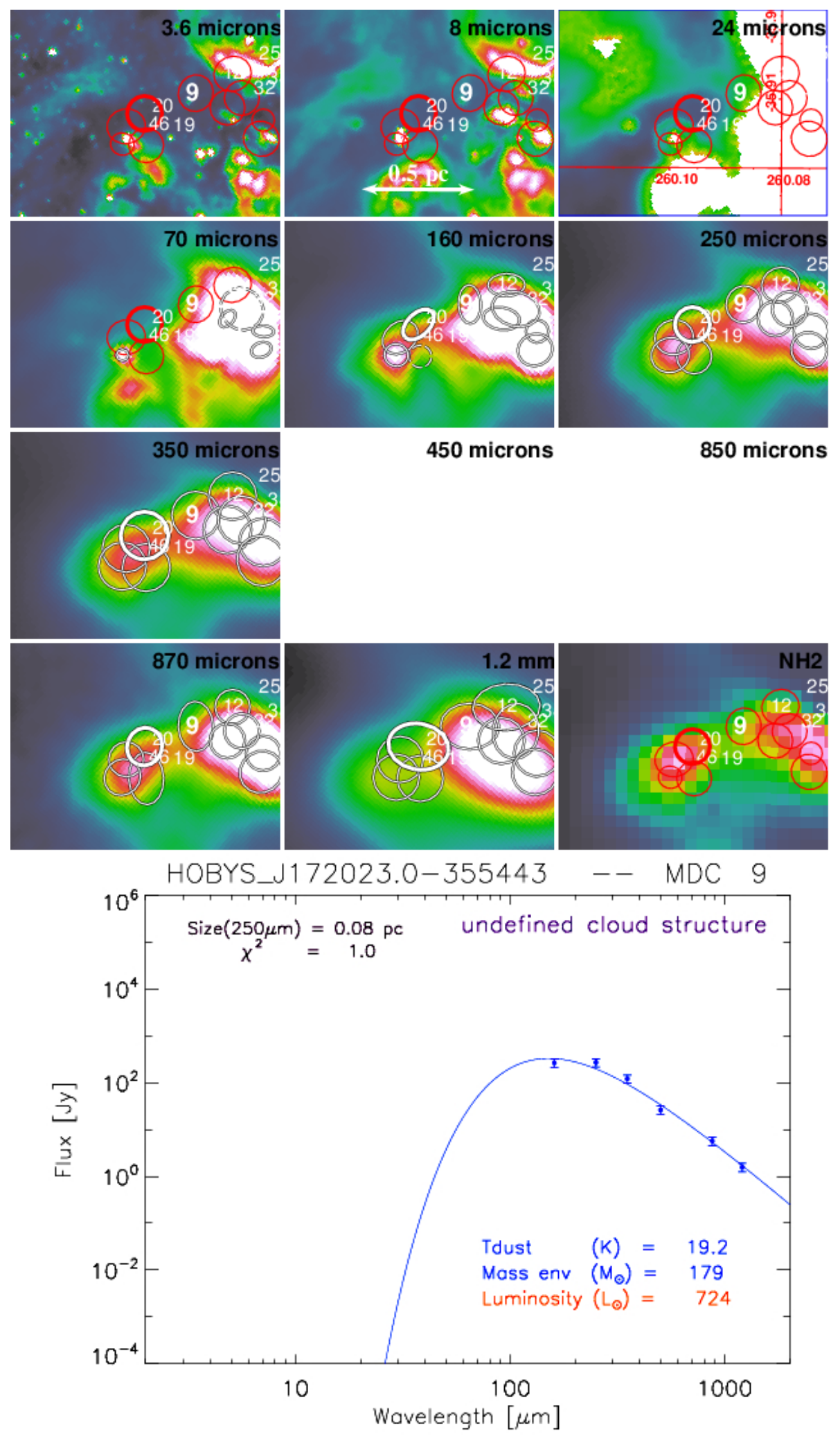

Fig. B.1. continued. 
J. Tigé et al.: The earliest phases of high-mass star formation in NGC 6334

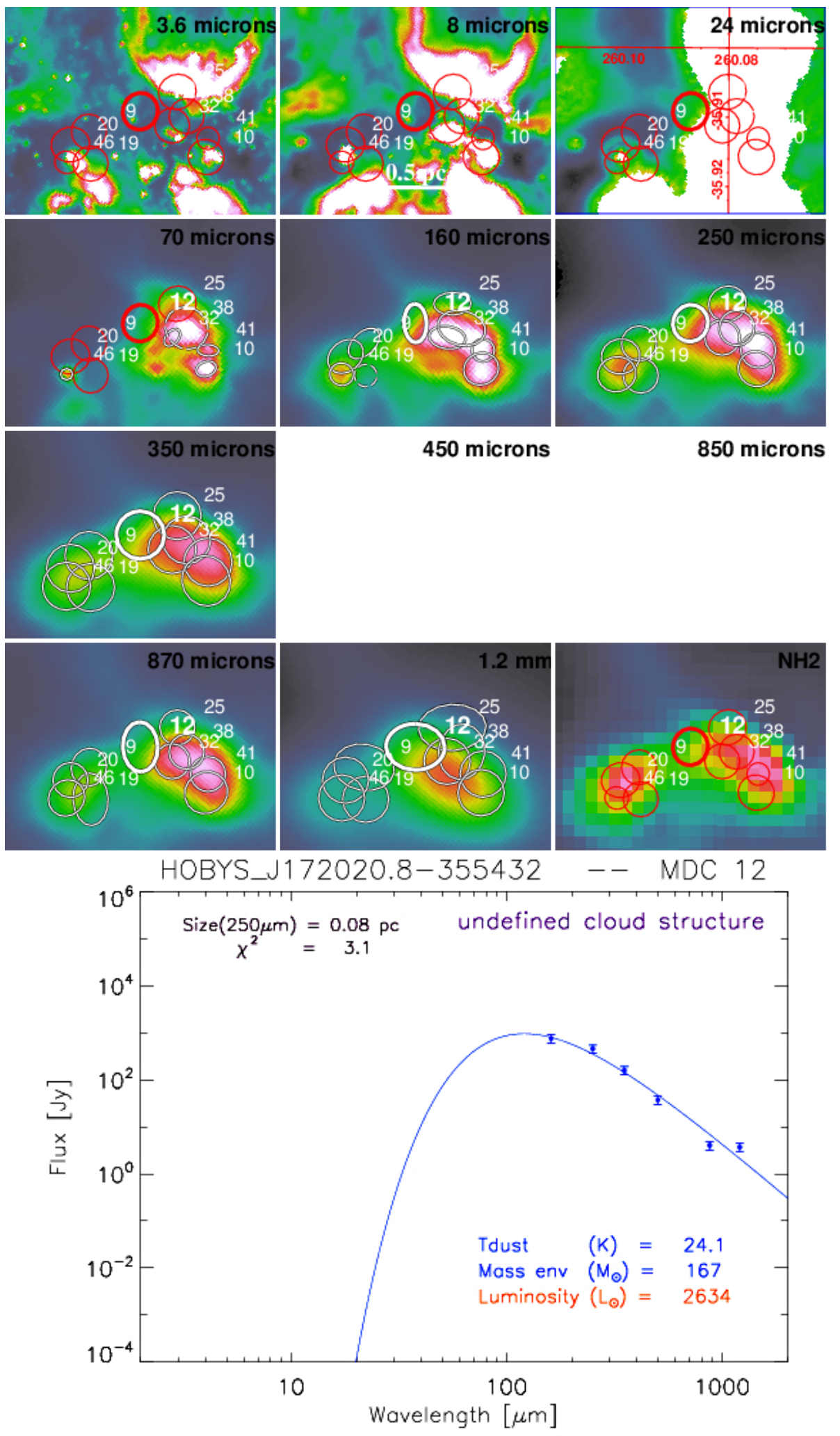

Fig. B.1. continued. 
A\&A 602, A77 (2017)

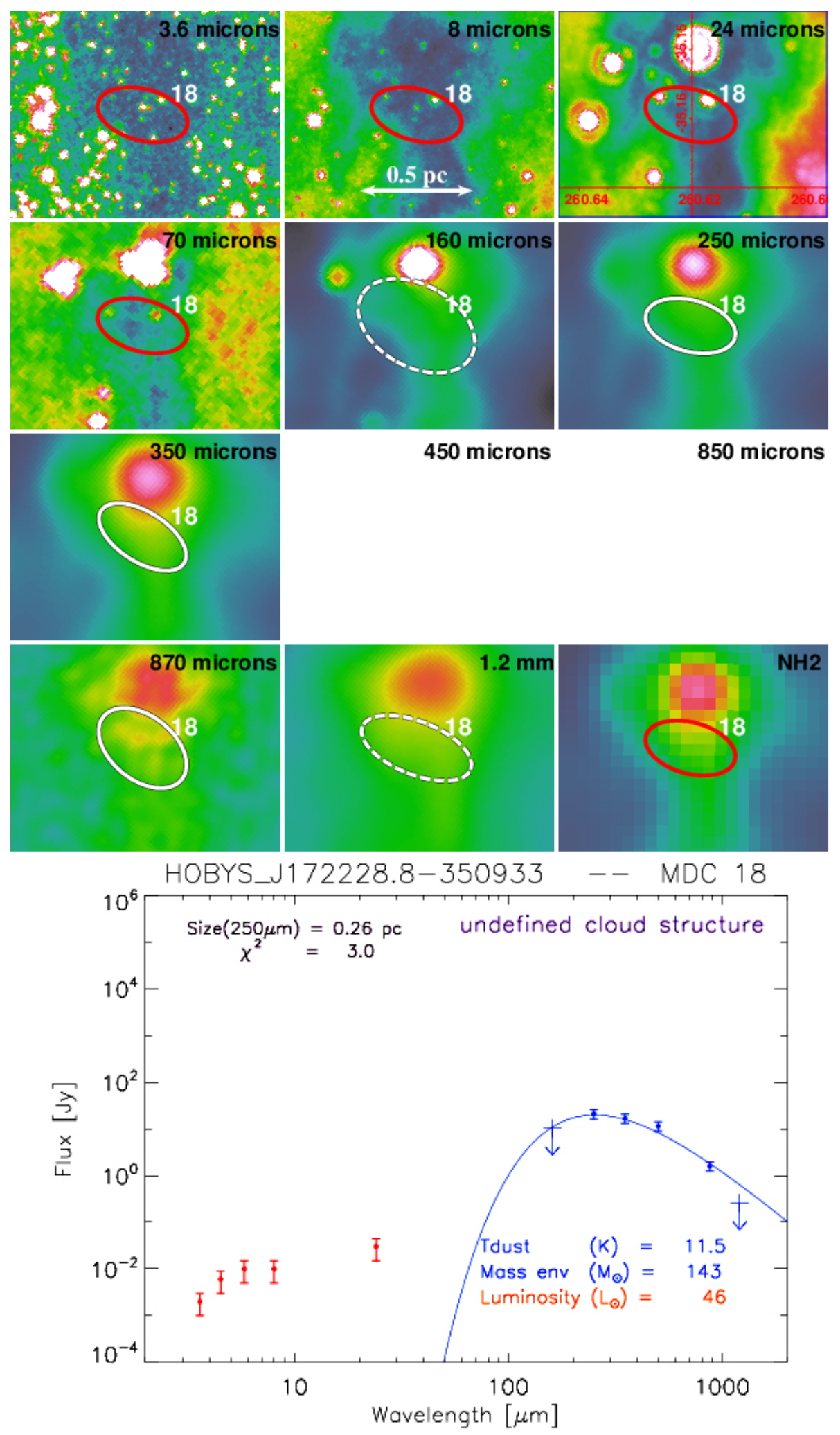

Fig. B.1. continued. 
J. Tigé et al.: The earliest phases of high-mass star formation in NGC 6334

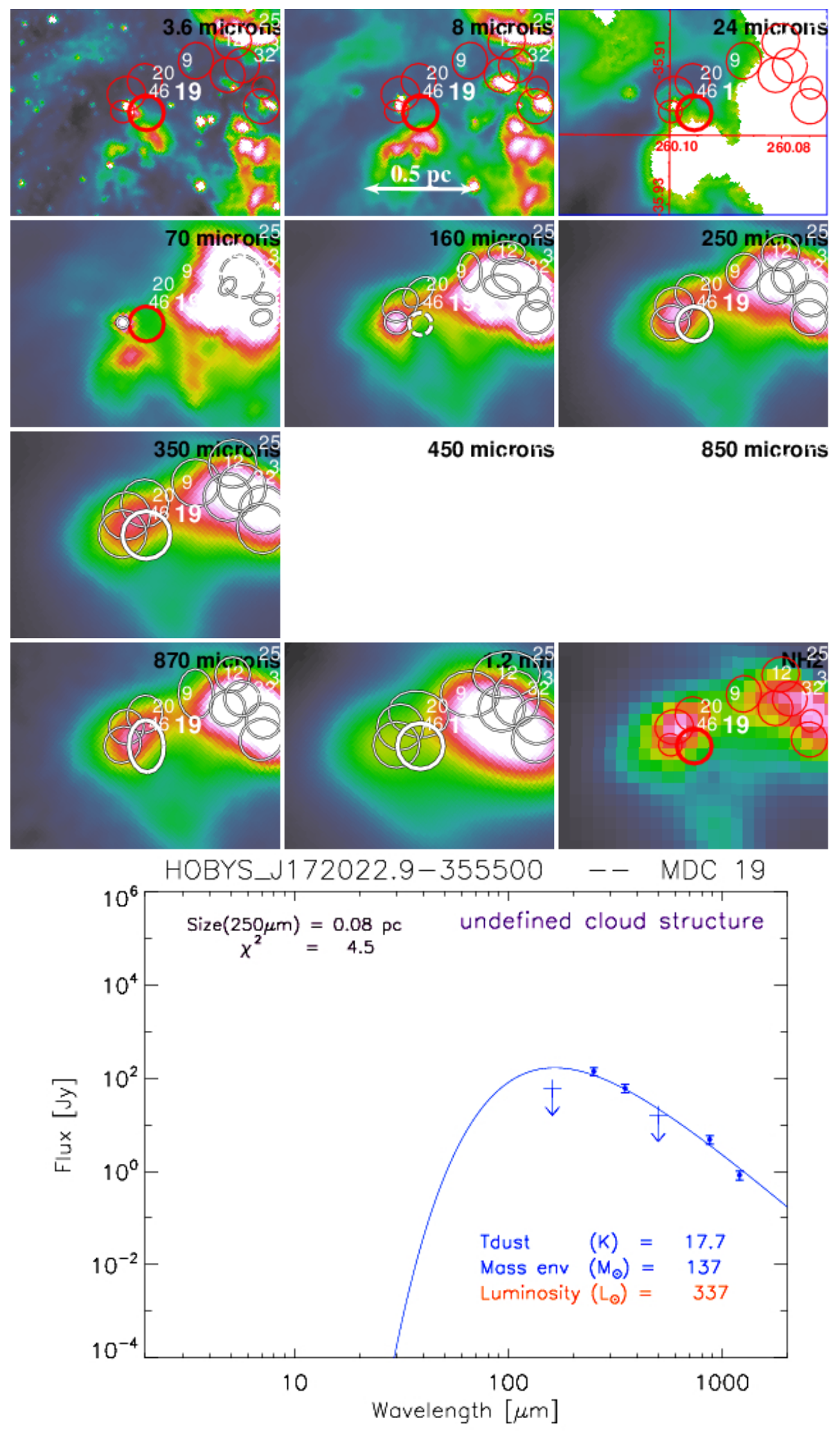

Fig. B.1. continued. 
A\&A 602, A77 (2017)

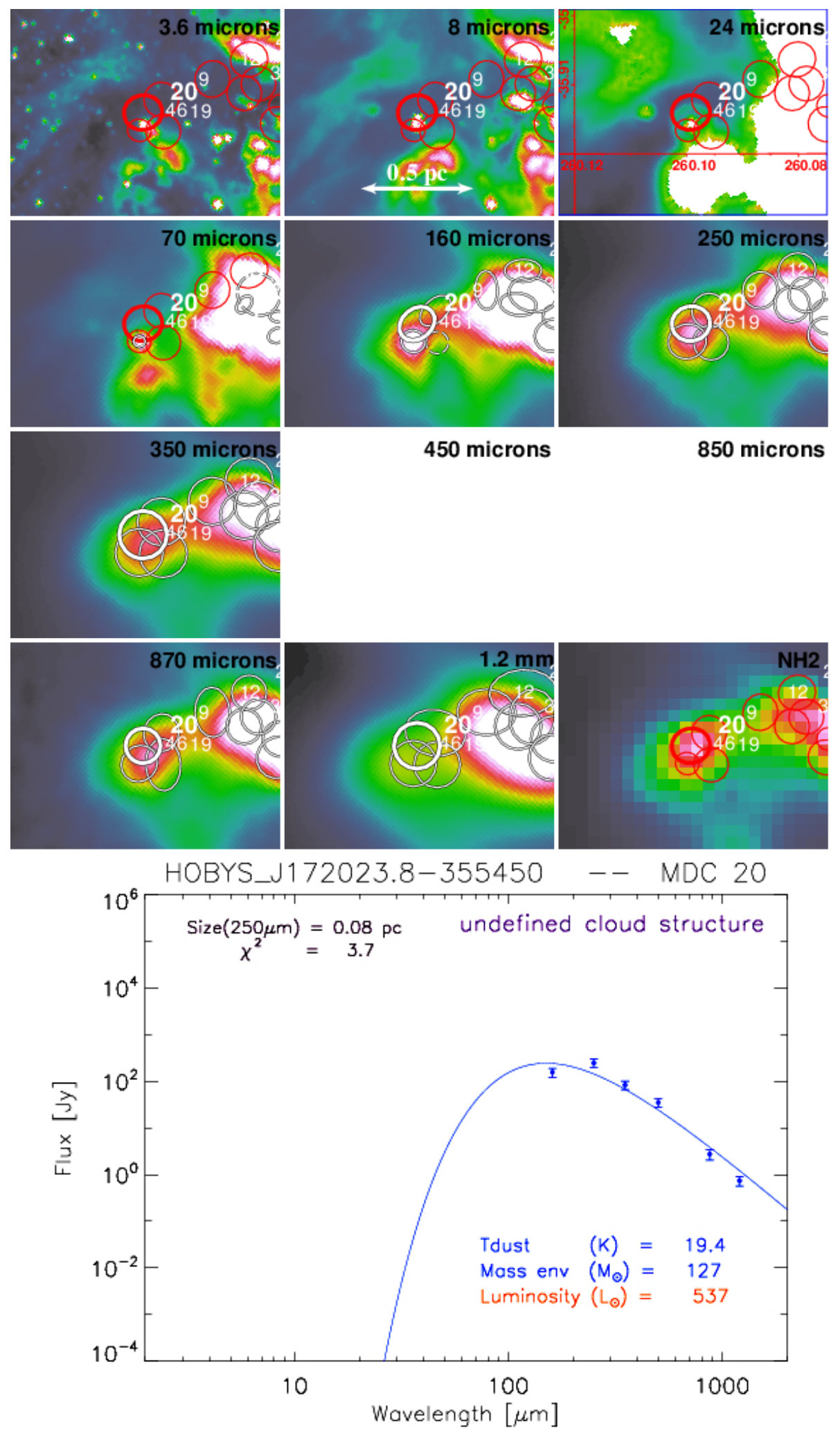

Fig. B.1. continued. 
J. Tigé et al.: The earliest phases of high-mass star formation in NGC 6334

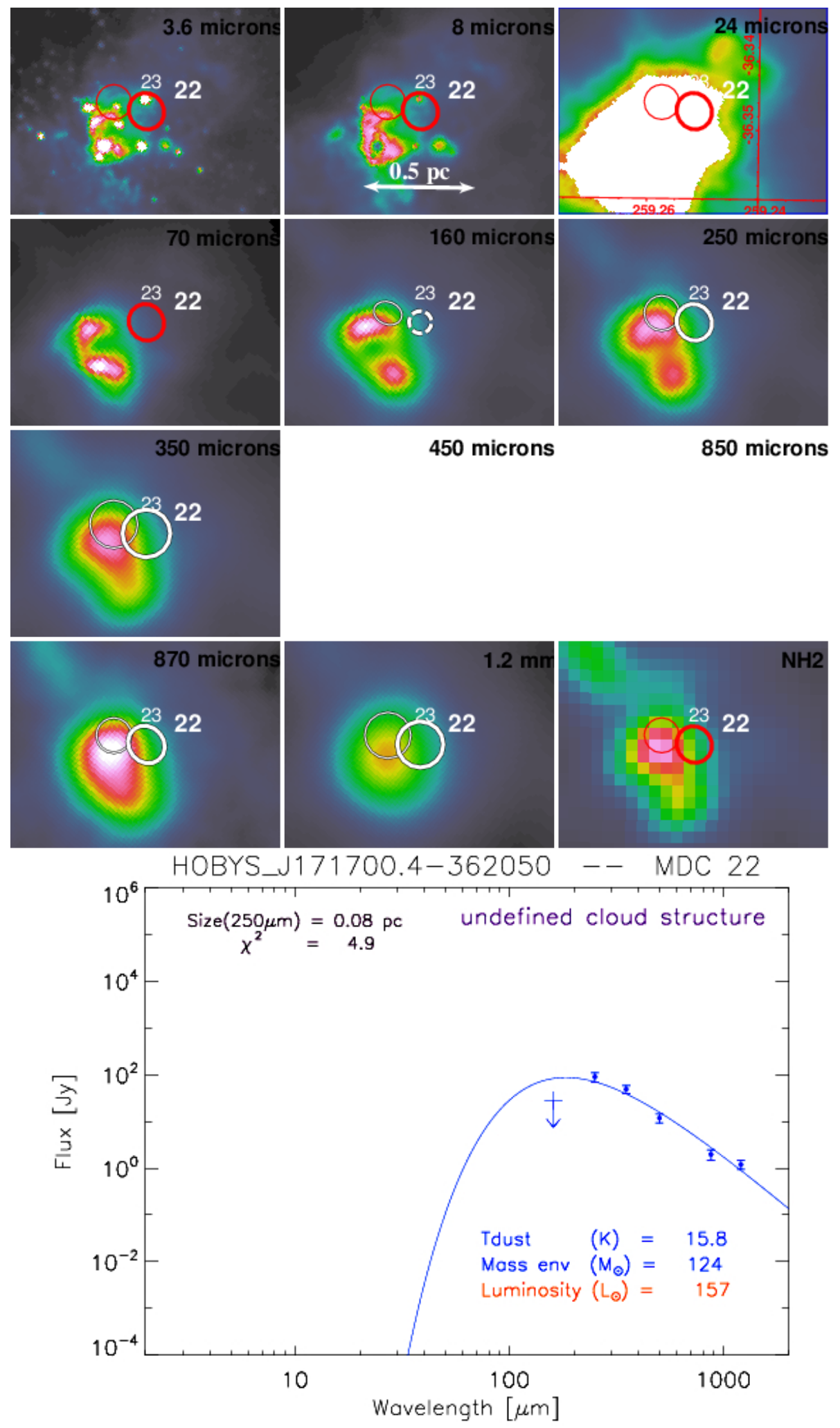

Fig. B.1. continued. 
A\&A 602, A77 (2017)

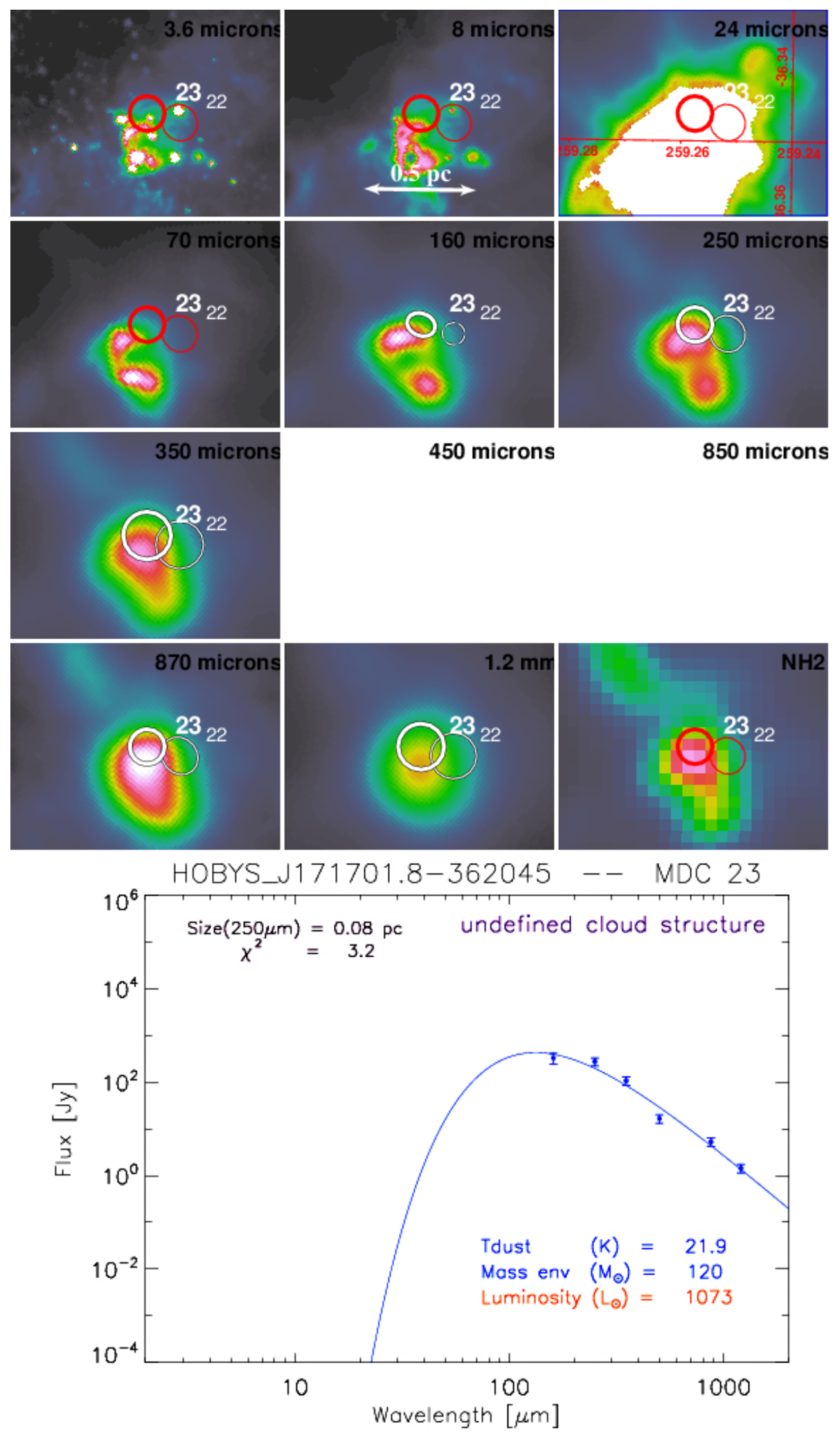

Fig. B.1. continued. 
J. Tigé et al.: The earliest phases of high-mass star formation in NGC 6334
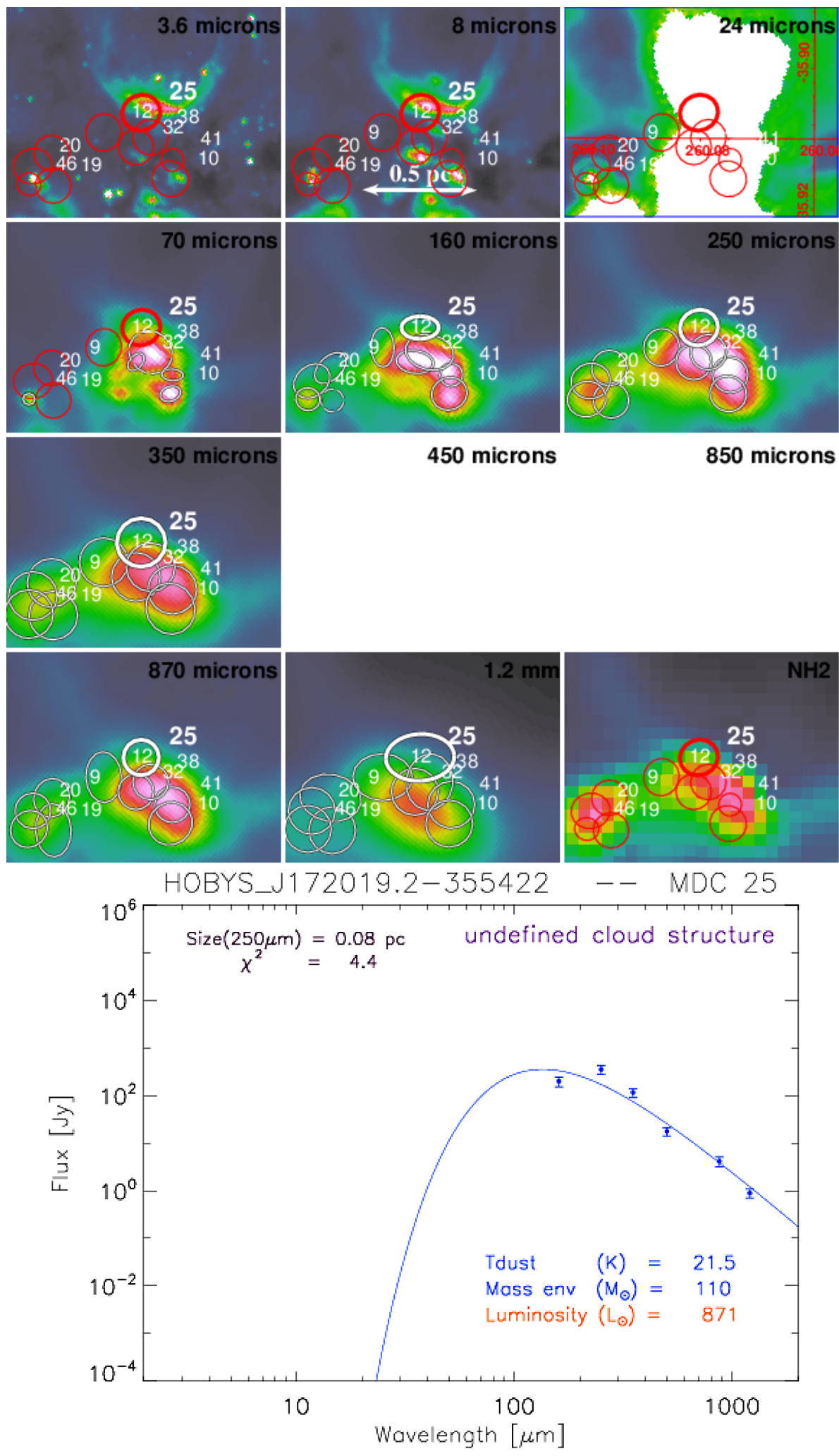

Fig. B.1. continued. 
A\&A 602, A77 (2017)

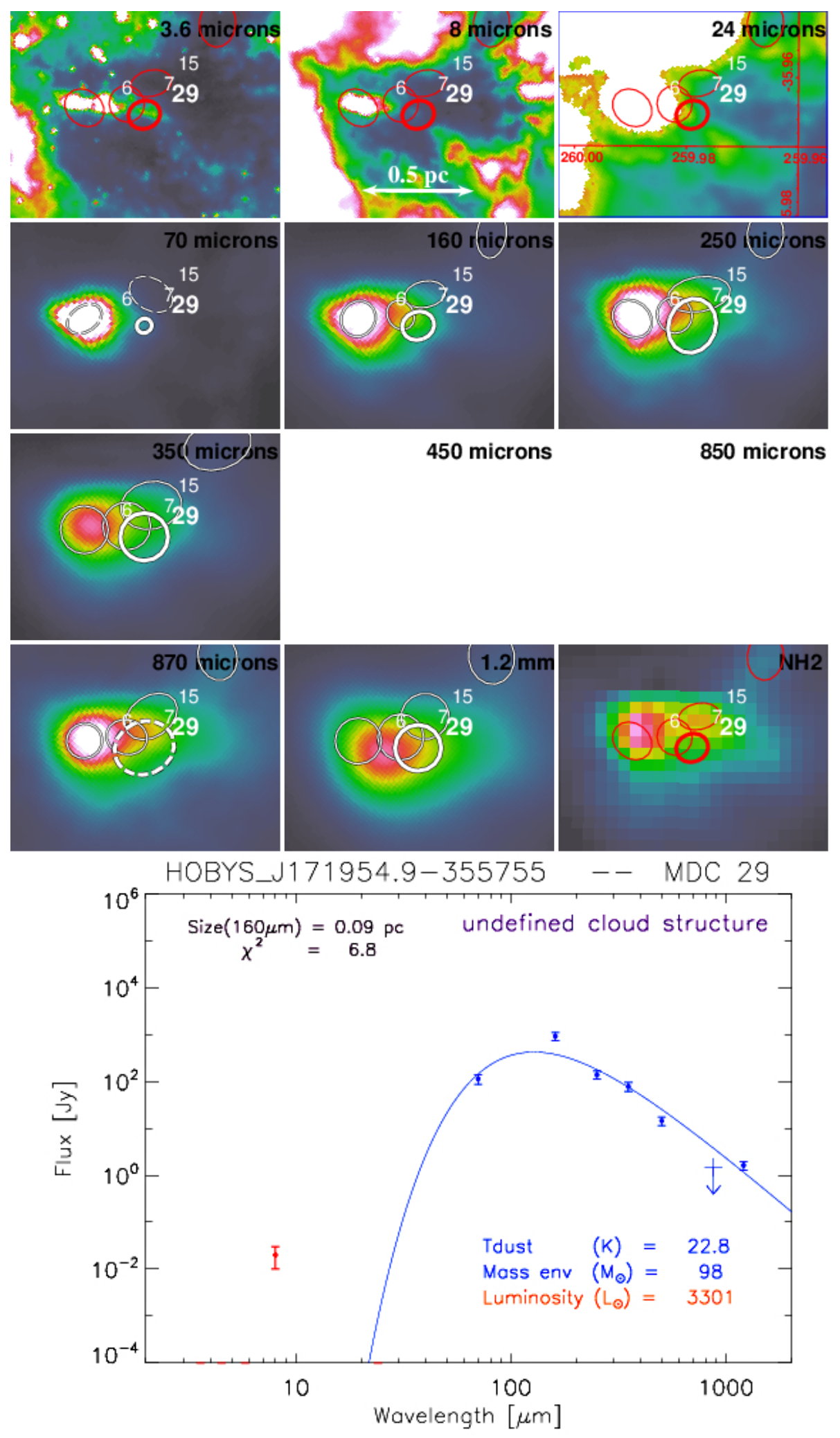

Fig. B.1. continued. 
J. Tigé et al.: The earliest phases of high-mass star formation in NGC 6334

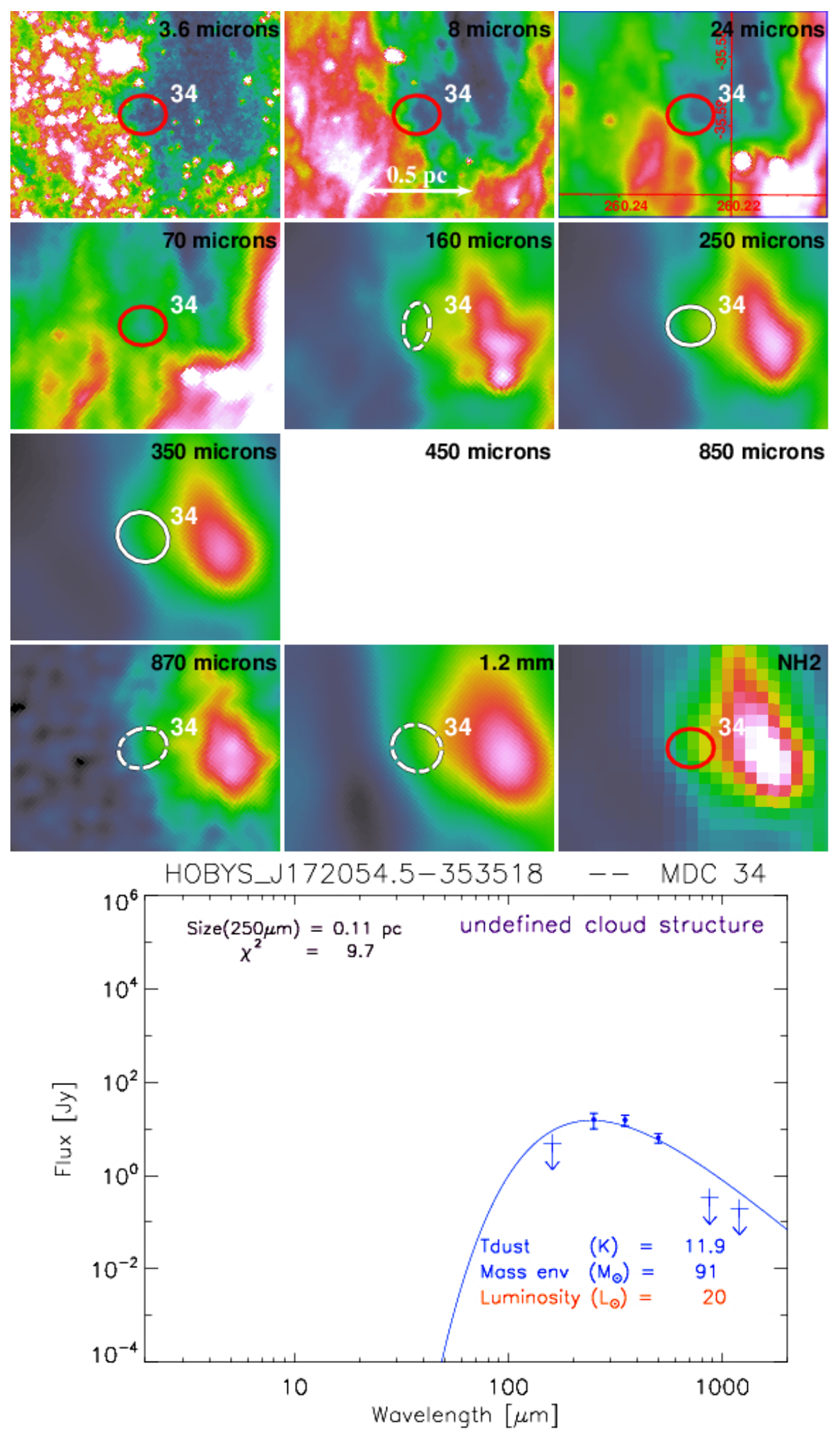

Fig. B.1. continued. 
A\&A 602, A77 (2017)

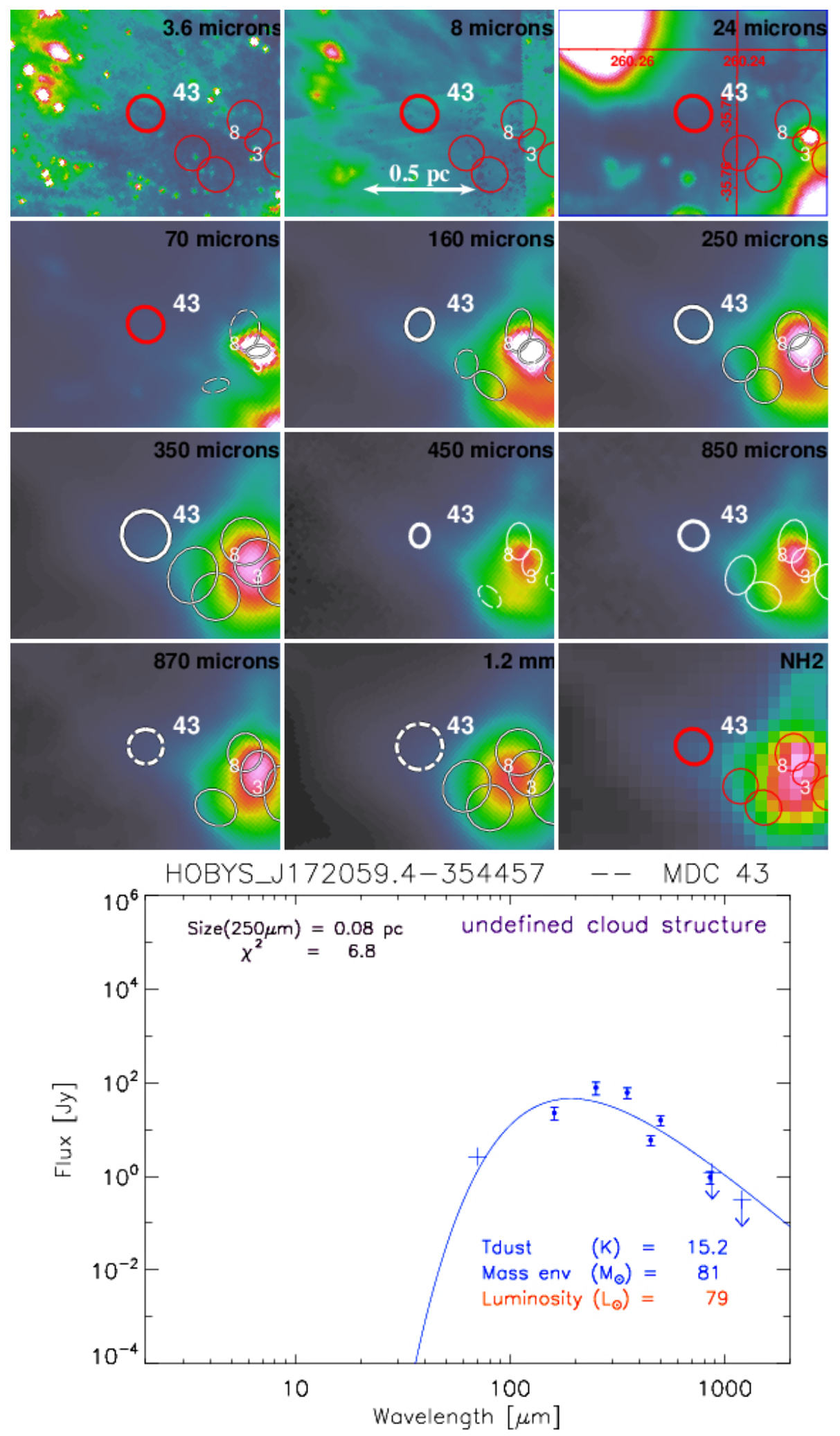

Fig. B.1. continued. 


\section{Appendix C: Images used for MDC extraction}

We extracted compact sources from Herschel images of Russeil et al. (2013) shown in Figs. C.1-C.5 and complementary images shown in Figs. C.6-C.12.

Figure C.6 presents the high-resolution column density map made using: 1) the procedure described in Appendix A of Palmeirim et al. (2013), based on a multi-scale decomposition of the imaging data; + and 2) the SED fitting Bayesian technique proposed by Hill et al. (2011).

Figures C.7 and C.8 present two mosaics toward the $I$ and $I(N)$ sources of NGC 6334 obtained from calibrated $450 \mu \mathrm{m}$ and $850 \mu \mathrm{m}$ SCUBA-2 data (see Sect. 2.2).

The PACS-160 $\mu \mathrm{m}$ and SPIRE-250 $\mu \mathrm{m}$ images are corrected from the effects of temperature gradients (see Figs. C.10-C.12). The purpose of the temperature-corrected maps at $160 \mu \mathrm{m}$ and $250 \mu \mathrm{m}$ is to weaken the impact of external heating, especially at the periphery of photo-dissociation regions to help getsources focus on column density peaks rather than emission peaks created by local heating.

To create the PACS- $160 \mu \mathrm{m}$ and SPIRE- $250 \mu \mathrm{m}$ temperaturecorrected maps we first derived a basic temperature map for the region, assuming a modified blackbody model with a spectral index of 2. The color temperature at each pixel was computed by the following equation (Wien approximation):

$T_{\text {col }}=\frac{h}{k} \times \frac{v_{160 \mu \mathrm{m}}-v_{250 \mu \mathrm{m}}}{\ln \left(\frac{S_{250 \mu \mathrm{m}}}{S_{160 \mu \mathrm{m}}} \times\left(\frac{v_{160 \mu \mathrm{m}}}{v_{250 \mu \mathrm{m}}}\right)^{5}\right)}$.

The resulting $T_{\text {col }}$ map was smoothed using a $40^{\prime \prime} \times 40^{\prime \prime}$ resolution median filter in order to trace temperature variations at larger scale. Flux weight-maps were then built, respectively at $160 \mu \mathrm{m}$ and $250 \mu \mathrm{m}$, as ratios between modified blackbody fluxes assuming a constant temperature and the color temperature of Eq. (C.1)

Weight-map $(160 \mu \mathrm{m})=\frac{S_{160 \mu \mathrm{m}}\left(T_{\mathrm{col}}\right)}{S_{160 \mu \mathrm{m}}(17 \mathrm{~K})}$

and

Weight-map $(250 \mu \mathrm{m})=\frac{S_{250 \mu \mathrm{m}}\left(T_{\mathrm{col}}\right)}{S_{250 \mu \mathrm{m}}(17 \mathrm{~K})}$.

We used a fiducial temperature of $17 \mathrm{~K}$, corresponding to the median temperature of Fig. C.9. Flux weight maps are ratio maps and therefore do not depend on the $N_{\mathrm{H}_{2}}$ distribution. We finally multiplied the original PACS-160 $\mu \mathrm{m}$ and SPIRE-250 $\mu \mathrm{m}$ images by their weight maps as follows:

$I_{\text {corrected }}(160 \mu \mathrm{m})=I_{160 \mu \mathrm{m}} \times$ Weight-map $(160 \mu \mathrm{m})$

and

$I_{\text {corrected }}(250 \mu \mathrm{m})=I_{250 \mu \mathrm{m}} \times$ Weight-map $(250 \mu \mathrm{m})$.

This weighting procedure artificially increases (decreases) the intensity of structures cooler (hotter) than $17 \mathrm{~K}$, thus improving the contrast of cold and compact cores, respectively, to the emission of more diffuse, heated cloud structures. Both the PACS$160 \mu \mathrm{m}$ and the SPIRE-250 $\mu \mathrm{m}$ temperature-corrected maps are used instead of the original PACS-160 $\mu \mathrm{m}$ and SPIRE-250 $\mu \mathrm{m}$ maps for the detection step in getsources. The temperaturecorrected images are shown in Figs. C.10 and C.11.

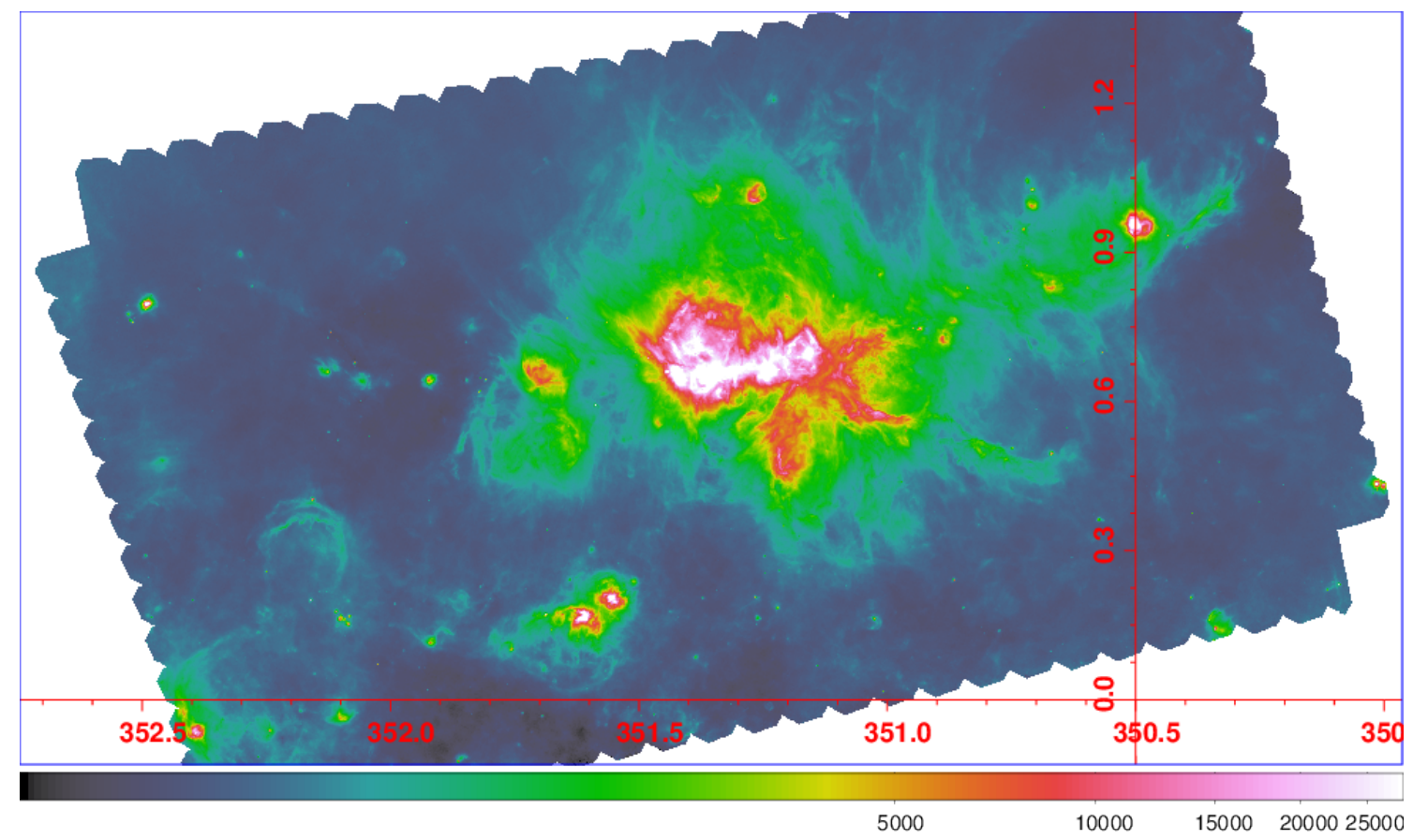

Fig. C.1. Herschel-HOBYS observations of NGC 6334 at $70 \mu \mathrm{m}$. Units are in MJy/sr. 
A\&A 602, A77 (2017)

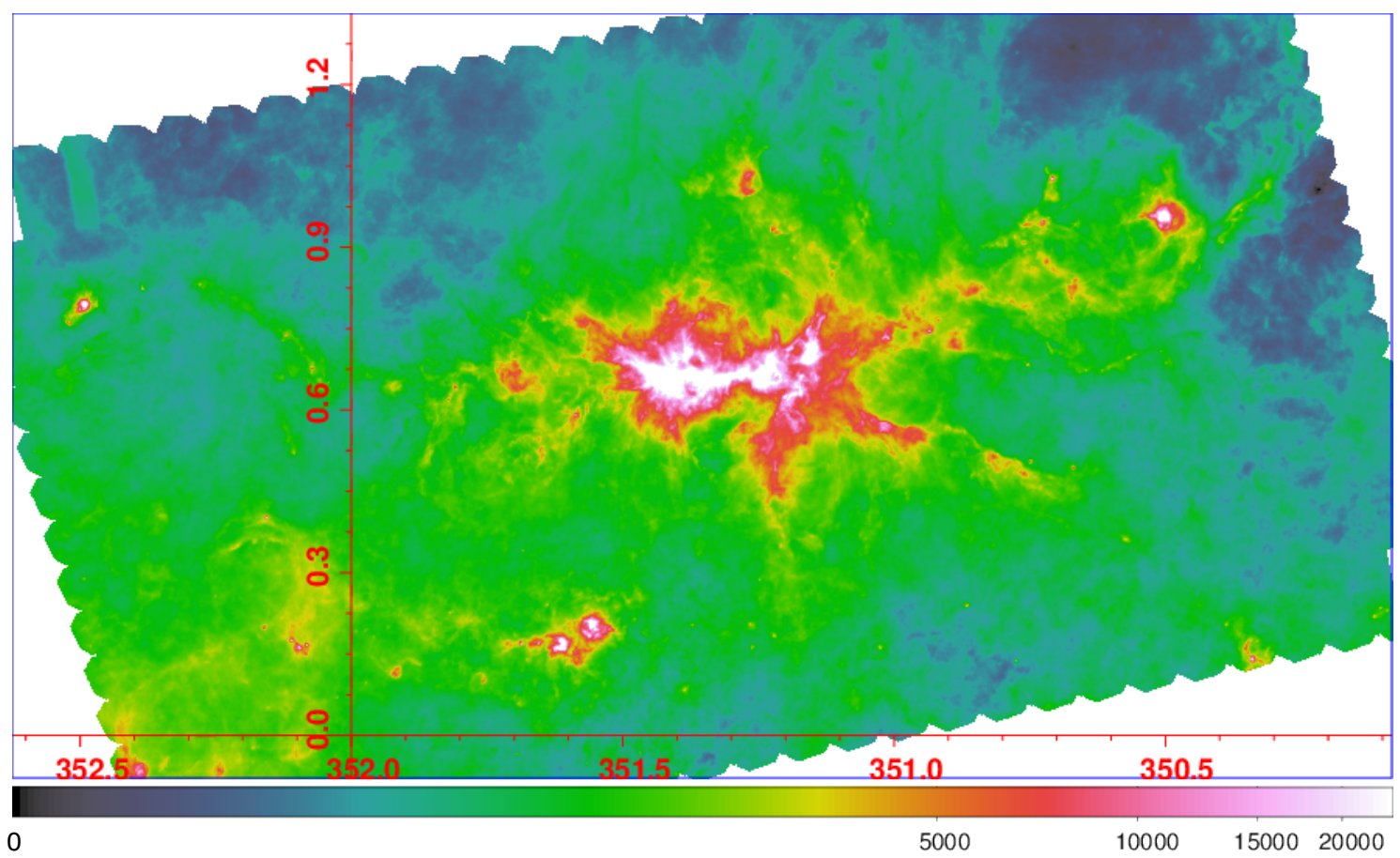

Fig. C.2. Herschel-HOBYS observations of NGC 6334 at $160 \mu \mathrm{m}$. Units are in MJy/sr.

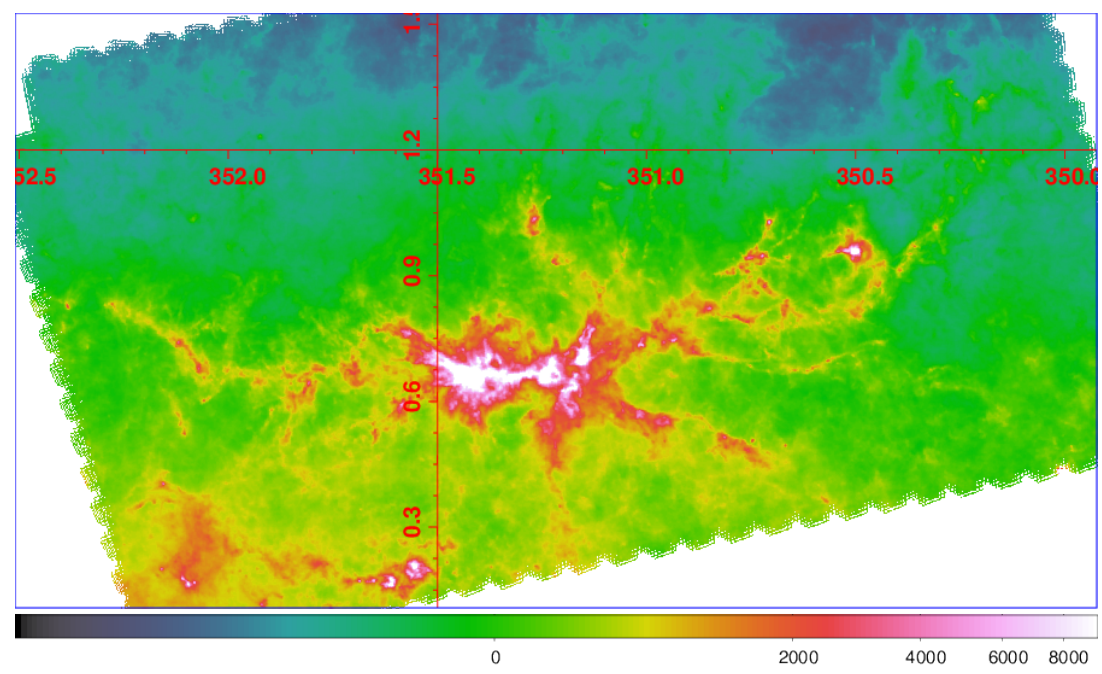

Fig. C.3. Herschel-HOBYS observations of NGC 6334 at $250 \mu \mathrm{m}$. Units are in MJy/sr. 
J. Tigé et al.: The earliest phases of high-mass star formation in NGC 6334

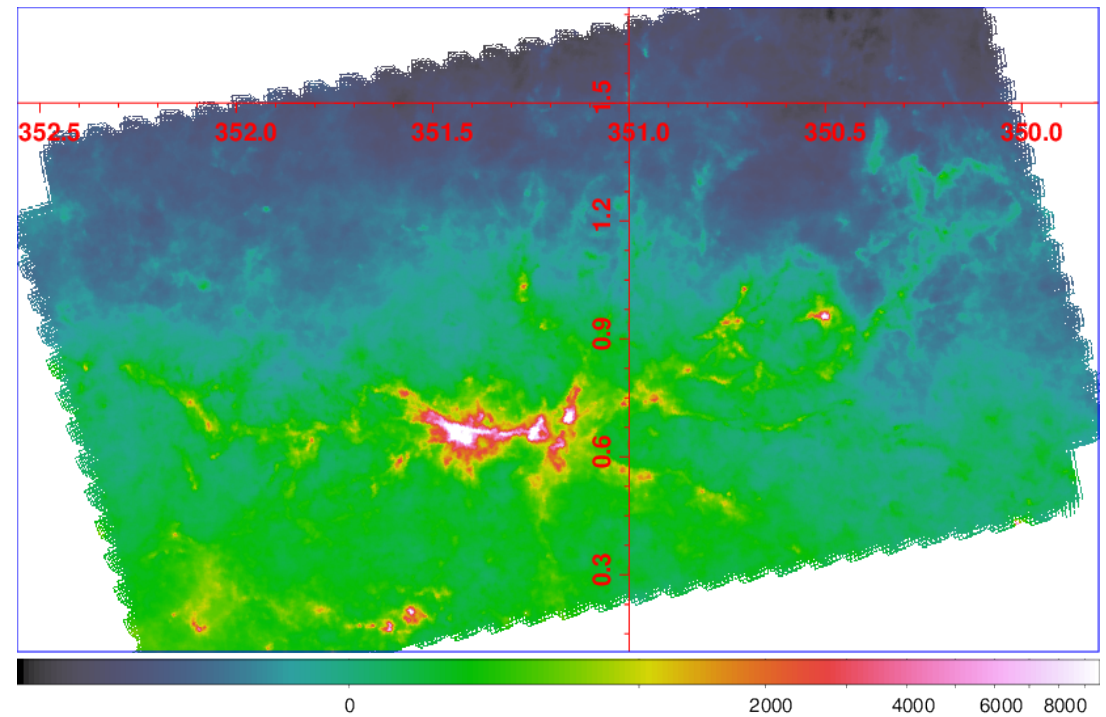

Fig. C.4. Herschel-HOBYS observations of NGC 6334 at $350 \mu \mathrm{m}$. Units are in MJy/sr.

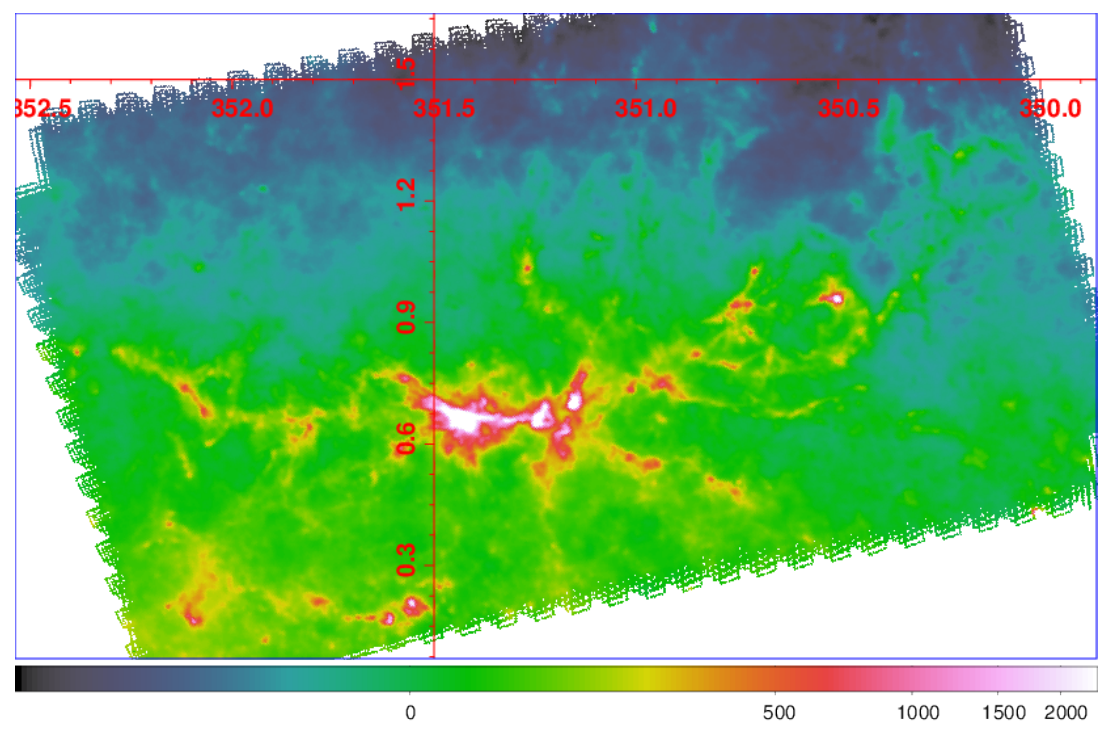

Fig. C.5. Herschel-HOBYS observations of NGC 6334 at $500 \mu \mathrm{m}$. Units are in MJy/sr.

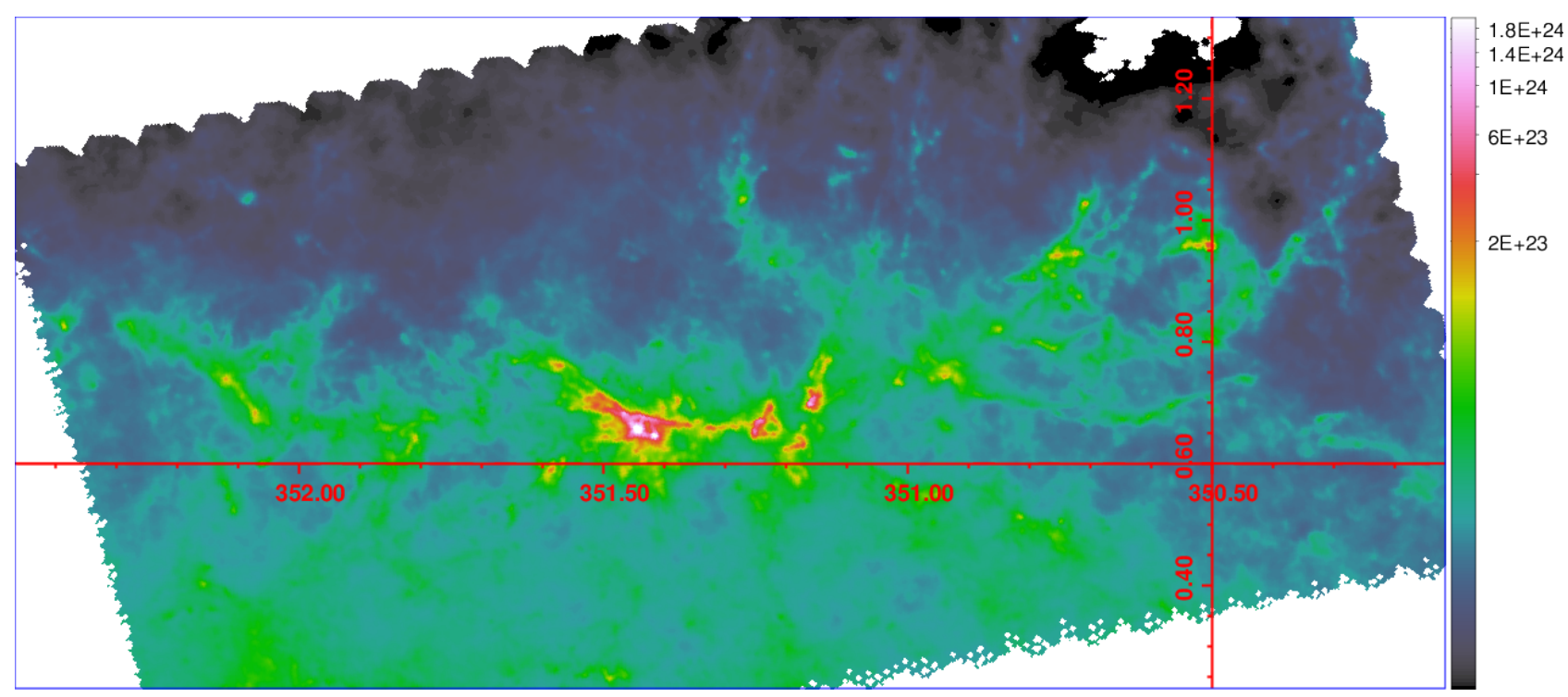

Fig. C.6. High-resolution column density map (resolution 18.2") of NGC 6334. colorbar is in log scale and units are $\mathrm{cm}^{-2}$. 
A\&A 602, A77 (2017)

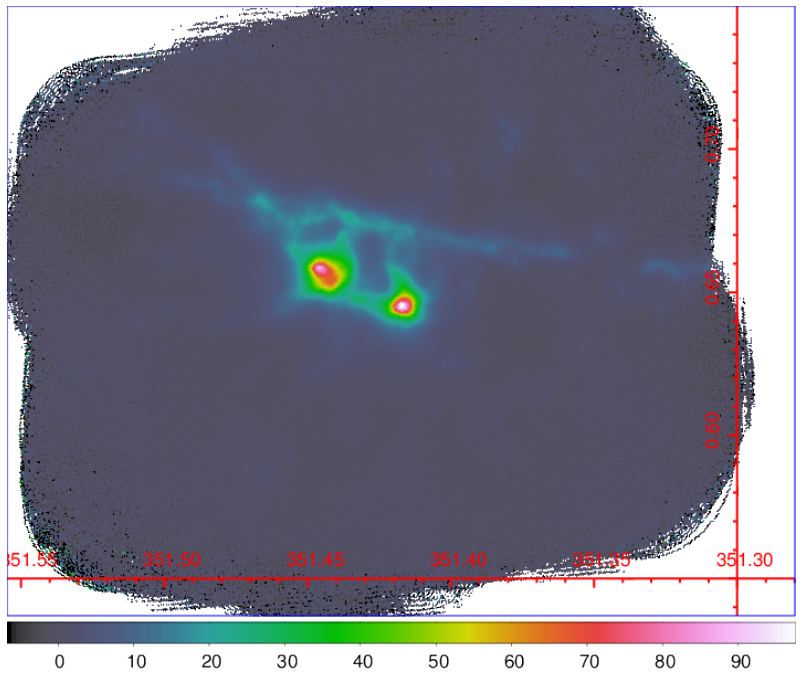

Fig. C.7. SCUBA-2 observations at $450 \mu \mathrm{m}$ toward the $I$ and $I(N)$ sources of NGC 6334. Units are in Jy/beam.

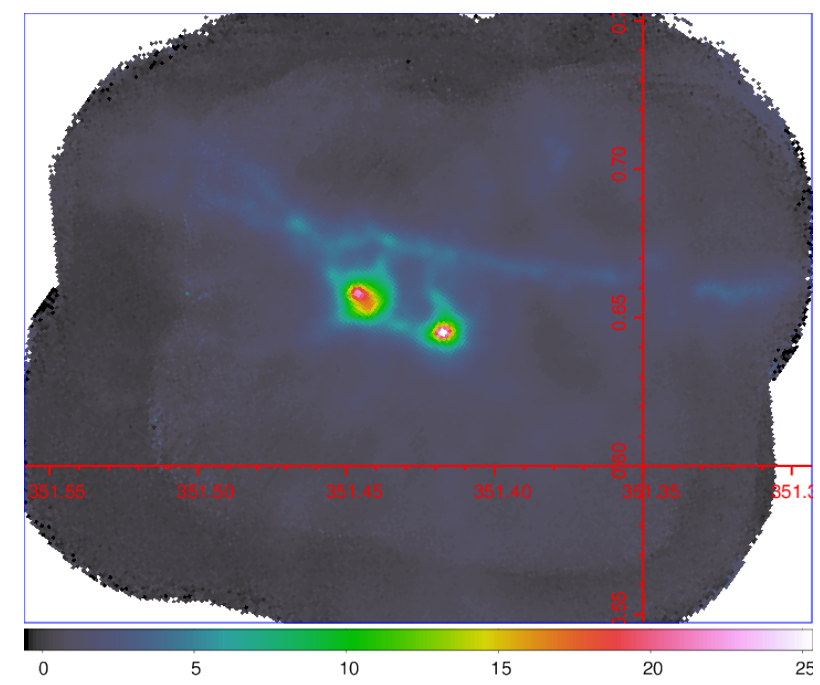

Fig. C.8. SCUBA-2 observations at $850 \mu \mathrm{m}$ toward the $I$ and $I(N)$ sources of NGC 6334. Units are in Jy/beam.

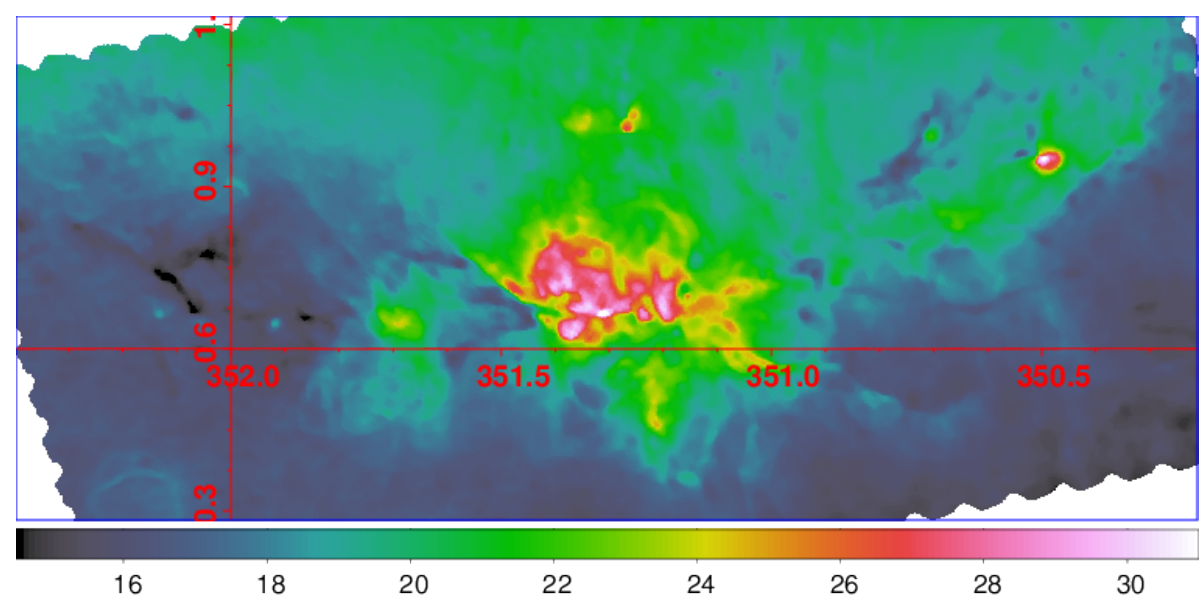

Fig. C.9. Herschel temperature $\left(T_{\mathrm{col}}\right)$ map of NGC 6334 derived using Eq. (C.1). Units are Kelvin degrees. 
J. Tigé et al.: The earliest phases of high-mass star formation in NGC 6334

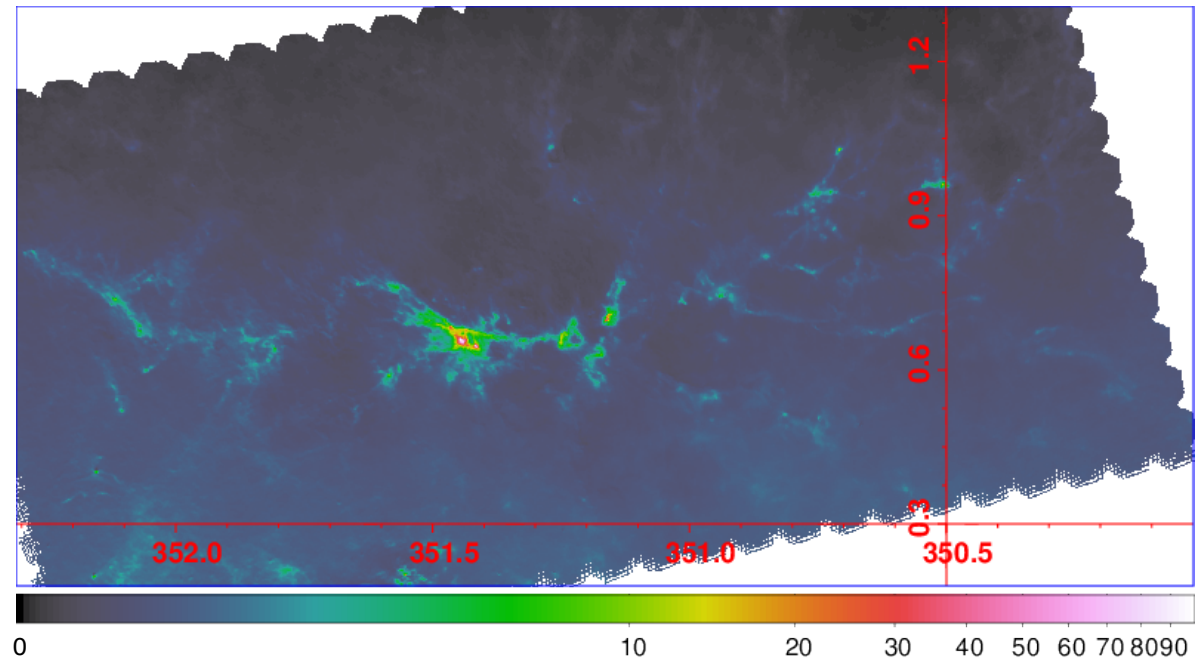

Fig. C.10. Herschel temperature-corrected map of NGC 6334 at $160 \mu \mathrm{m}$. Units are in $\%$ of the maximum flux.

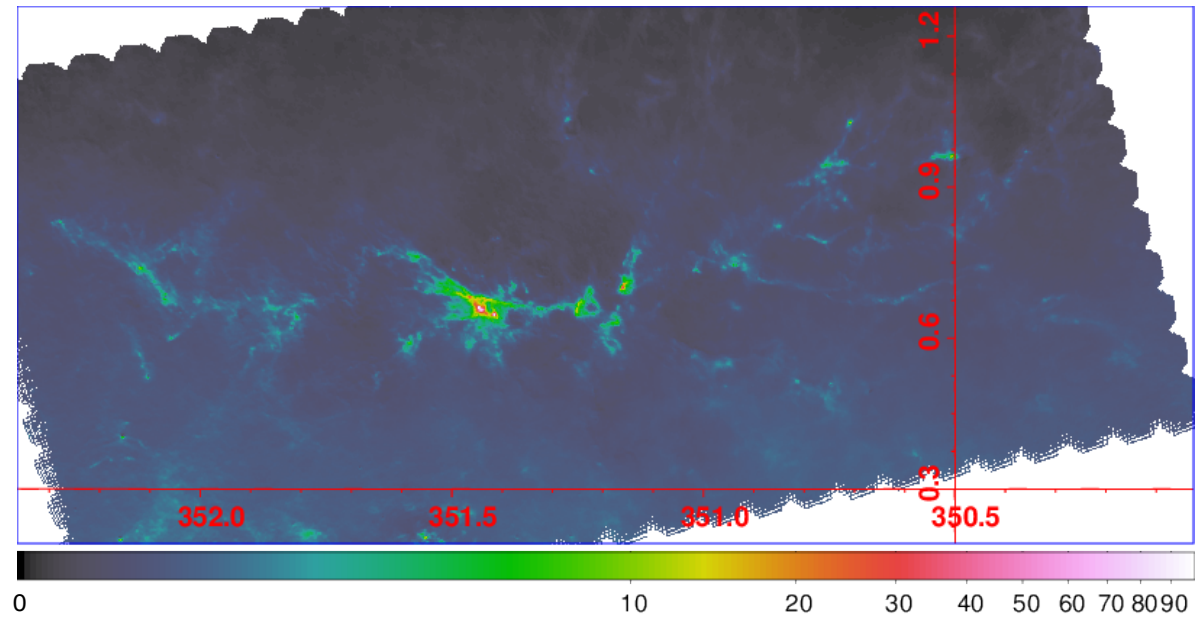

Fig. C.11. Herschel temperature-corrected map of NGC 6334 at $250 \mu \mathrm{m}$. Units are in $\%$ of the maximum flux.
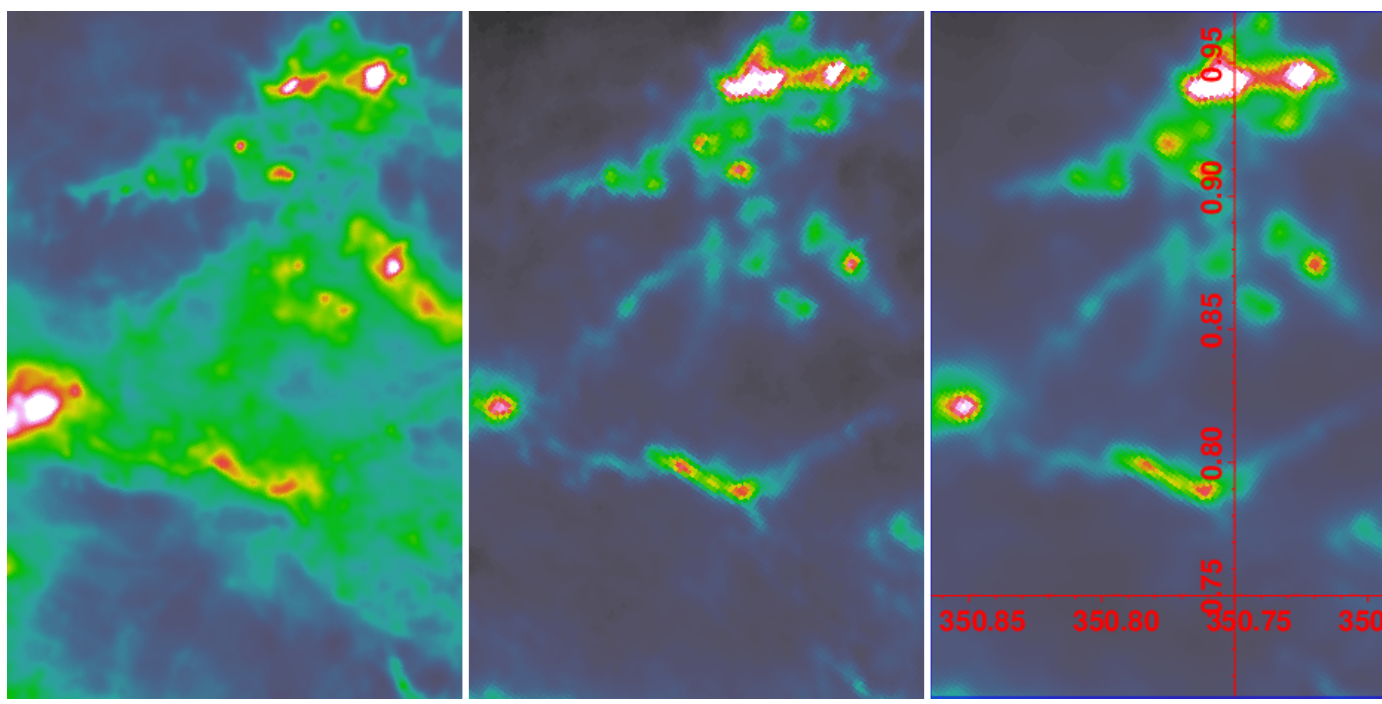

Fig. C.12. Removing temperature gradient: from left to right, Herschel/PACS $160 \mu \mathrm{m}$, Herschel/PACS temperature-corrected map at $160 \mu \mathrm{m}$, and high-resolution column density map. 